\title{
Interventions for basal cell carcinoma of the skin (Review)
}

Thomson J, Hogan S, Leonardi-Bee J, Williams HC, Bath-Hextall FJ

Thomson J, Hogan S, Leonardi-Bee J, Williams HC, Bath-Hextall FJ.

Interventions for basal cell carcinoma of the skin.

Cochrane Database of Systematic Reviews 2020, Issue 11. Art. No.: CD003412.

DOI: 10.1002/14651858.CD003412.pub3.

www.cochranelibrary.com 
TABLE OF CONTENTS

HEADER

ABSTRACT

PLAIN LANGUAGE SUMMARY

SUMMARY OF FINDINGS

BACKGROUND

OBJECTIVES

METHODS

RESULTS

Figure 1.

Figure 2.

Figure 3.

DISCUSSION

AUTHORS' CONCLUSIONS

ACKNOWLEDGEMENTS

REFERENCES

CHARACTERISTICS OF STUDIES

DATA AND ANALYSES

Analysis 1.1. Comparison 1: Mohs micrographic surgery vs surgical excision, Outcome 1: Recurrence at 3 years (lesions) ........

Analysis 1.2. Comparison 1: Mohs micrographic surgery vs surgical excision, Outcome 2: Recurrence at 5 years (lesions) ........

Analysis 2.1. Comparison 2: Imiquimod vs surgical excision, Outcome 1: Recurrence at 3 years

Analysis 2.2. Comparison 2: Imiquimod vs surgical excision, Outcome 2: Recurrence at 5 years

Analysis 2.3. Comparison 2: Imiquimod vs surgical excision, Outcome 3: Cosmetic outcome (good/excellent)

Analysis 2.4. Comparison 2: Imiquimod vs surgical excision, Outcome 4: Pain (moderate/severe)

Analysis 3.1. Comparison 3: Radiotherapy vs surgical excision (with or without frozen section margin control), Outcome 1: Recurrence at 3 years

Analysis 3.2. Comparison 3: Radiotherapy vs surgical excision (with or without frozen section margin control), Outcome 2: Recurrence at 4 years

Analysis 3.3. Comparison 3: Radiotherapy vs surgical excision (with or without frozen section margin control), Outcome 3: Cosmetic outcome (good)

Analysis 4.1. Comparison 4: Curettage vs surgical excision, Outcome 1: Recurrence at 2 years (lesions)

Analysis 5.1. Comparison 5: Curettage and cautery vs surgical excision, Outcome 1: Recurrence at 2 years (lesions) ..................

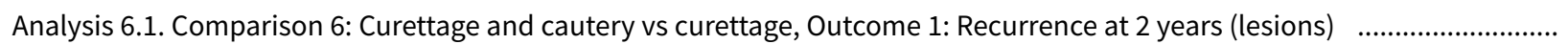

Analysis 7.1. Comparison 7: Low dose radiotherapy vs high dose radiotherapy, Outcome 1: Early treatment failure .................

Analysis 8.1. Comparison 8: Radiotherapy vs cryosurgery, Outcome 1: Recurrence at 2 years

Analysis 9.1. Comparison 9: Curettage and cryosurgery vs surgical excision, Outcome 1: Recurrence at 3 years (lesions) .........

Analysis 9.2. Comparison 9: Curettage and cryosurgery vs surgical excision, Outcome 2: Recurrence at 5 years (lesions) .........

Analysis 9.3. Comparison 9: Curettage and cryosurgery vs surgical excision, Outcome 3: Cosmetic outcome (good) ................

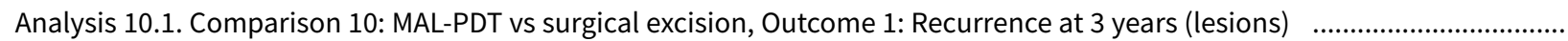

Analysis 10.2. Comparison 10: MAL-PDT vs surgical excision, Outcome 2: Cosmetic outcome (good/excellent) .........................

Analysis 10.3. Comparison 10: MAL-PDT vs surgical excision, Outcome 3: Pain

Analysis 10.4. Comparison 10: MAL-PDT vs surgical excision, Outcome 4: Early treatment failure (lesions) …...........................

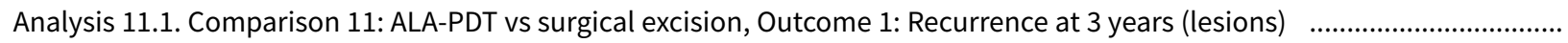

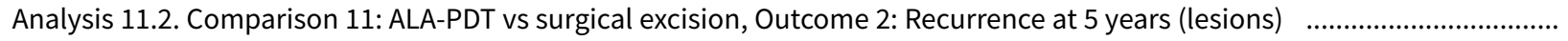

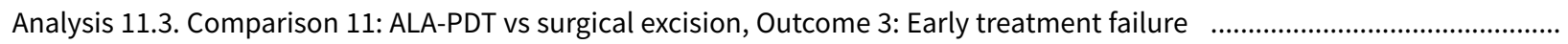

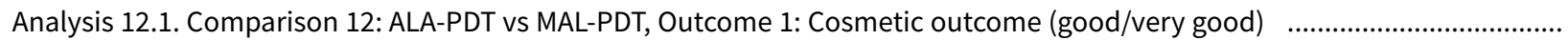

Analysis 12.2. Comparison 12: ALA-PDT vs MAL-PDT, Outcome 2: Pain (visual analogue score)

Analysis 12.3. Comparison 12: ALA-PDT vs MAL-PDT, Outcome 3: Pain (numerical rating scale)

Analysis 12.4. Comparison 12: ALA-PDT vs MAL-PDT, Outcome 4: Early treatment failure

Analysis 13.1. Comparison 13: BF-200 ALA-PDT vs MAL-PDT, Outcome 1: Early treatment failure

Analysis 14.1. Comparison 14: HAL-PDT vs MAL-PDT, Outcome 1: Early treatment failure

Analysis 15.1. Comparison 15: HAL-PDT vs BF-200 ALA-PDT, Outcome 1: Early treatment failure 


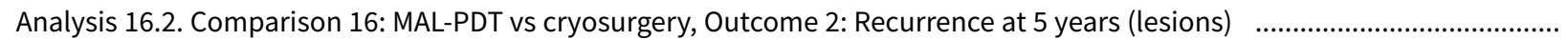

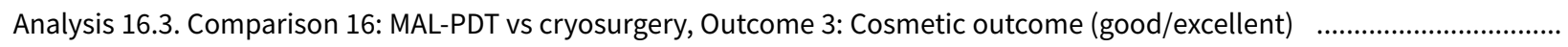
Analysis 16.4. Comparison 16: MAL-PDT vs cryosurgery, Outcome 4: Pain

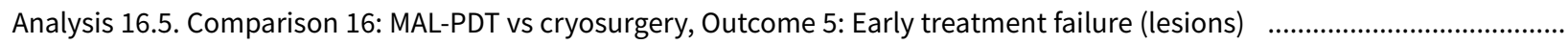
Analysis 17.1. Comparison 17: ALA-PDT vs cryosurgery, Outcome 1: Cosmetic outcome (good/excellent) ................................ Analysis 17.2. Comparison 17: ALA-PDT vs cryosurgery, Outcome 2: Pain Analysis 18.1. Comparison 18: Imiquimod cream vs MAL-PDT, Outcome 1: Recurrence at 3 years Analysis 18.2. Comparison 18: Imiquimod cream vs MAL-PDT, Outcome 2: Recurrence at 5 years Analysis 18.3. Comparison 18: Imiquimod cream vs MAL-PDT, Outcome 3: Cosmetic outcome (good/excellent, observer rated at 1 year)

Analysis 18.4. Comparison 18: Imiquimod cream vs MAL-PDT, Outcome 4: Pain (moderate/severe)

Analysis 18.5. Comparison 18: Imiquimod cream vs MAL-PDT, Outcome 5: Early treatment failure

Analysis 19.1. Comparison 19: 5-FU cream vs MAL-PDT, Outcome 1: Recurrence at 3 years

Analysis 19.2. Comparison 19: 5-FU cream vs MAL-PDT, Outcome 2: Recurrence at 5 years

Analysis 19.3. Comparison 19: 5-FU cream vs MAL-PDT, Outcome 3: Cosmetic outcome (good/excellent, observer rated at 1 year)

Analysis 19.4. Comparison 19: 5-FU cream vs MAL-PDT, Outcome 4: Pain (moderate/severe)

Analysis 19.5. Comparison 19: 5-FU cream vs MAL-PDT, Outcome 5: Early treatment failure

Analysis 20.1. Comparison 20: Laser ALA-PDT vs broadband light ALA-PDT, Outcome 1: Cosmetic outcome (good/excellent, observer rated at 6 months)

Analysis 20.2. Comparison 20: Laser ALA-PDT vs broadband light ALA-PDT, Outcome 2: Pain

Analysis 21.1. Comparison 21: AFXL MAL-PDT vs MAL-PDT, Outcome 1: Cosmetic outcome (good/excellent)

Analysis 21.2. Comparison 21: AFXL MAL-PDT vs MAL-PDT, Outcome 2: Early treatment failure

Analysis 22.1. Comparison 22: MAL-PDT vs placebo, Outcome 1: Cosmetic outcome (good/excellent, observer rated at 6 months))

Analysis 22.2. Comparison 22: MAL-PDT vs placebo, Outcome 2: Pain (frequency reported as local AE)

Analysis 22.3. Comparison 22: MAL-PDT vs placebo, Outcome 3: Early treatment failure (lesions)

Analysis 23.1. Comparison 23: Imiquimod cream vs 5-FU cream, Outcome 1: Recurrence at 3 years

Analysis 23.2. Comparison 23: Imiquimod cream vs 5-FU cream, Outcome 2: Recurrence at 5 years

Analysis 23.3. Comparison 23: Imiquimod cream vs 5-FU cream, Outcome 3: Cosmetic outcome (good/excellent, observer rated at 1 year)

Analysis 23.4. Comparison 23: Imiquimod cream vs 5-FU cream, Outcome 4: Pain (moderate/severe)

Analysis 23.5. Comparison 23: Imiquimod cream vs 5-FU cream, Outcome 5: Early treatment failure

Analysis 24.1. Comparison 24: Imiquimod vs radiotherapy, Outcome 1: Cosmetic outcome (excellent, participant rated at 6 weeks)

Analysis 25.1. Comparison 25: Imiquimod cream under occlusion vs no occlusion, Outcome 1: Early treatment failure

Analysis 26.1. Comparison 26: Imiquimod cream vs vehicle, Outcome 1: Early treatment failure

Analysis 27.1. Comparison 27: Imiquimod 5 weeks vs 8 weeks, Outcome 1: Recurrence at 1 year

Analysis 28.1. Comparison 28: Imiquimod 8 weeks vs 12 weeks, Outcome 1: Early treatment failure

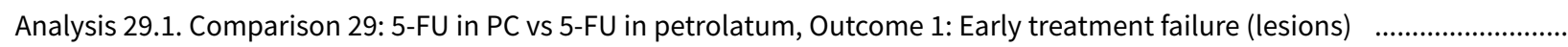

Analysis 30.1. Comparison 30: 5-FU/epinephrine gel (1.0 mL once a week for 6 weeks vs $0.5 \mathrm{~mL}$ once a week for 6 weeks, Outcome 1: Early treatment failure

Analysis 31.1. Comparison 31: 5-FU/epinephrine gel(1.0 mL twice a week for 3 weeks vs $0.5 \mathrm{~mL}$ twice a week for 3 weeks), Outcome 1: Early treatment failure

Analysis 32.1. Comparison 32: 5-FU/epinephrine gel( $0.5 \mathrm{ml}$ twice a week for 4 weeks vs $0.5 \mathrm{~mL}$ three times a week for 2 weeks., Outcome 1: Early treatment failure

Analysis 33.1. Comparison 33: Electrochemotherapy vs surgical excision, Outcome 1: Recurrence at 3 years ...........................

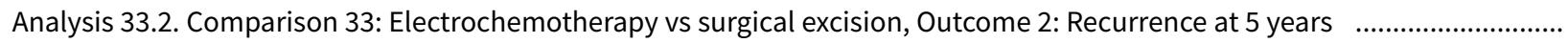

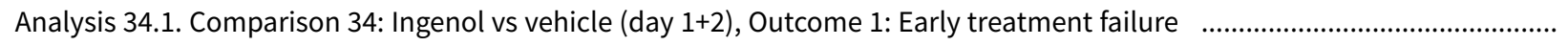

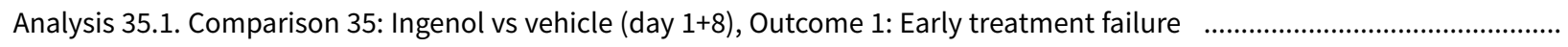
Analysis 36.1. Comparison 36: Ingenol under occlusion vs no occlusion, Outcome 1: Early treatment failure 


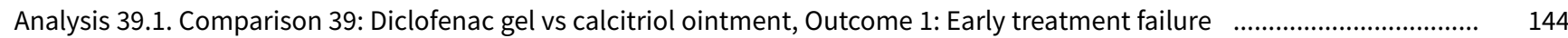

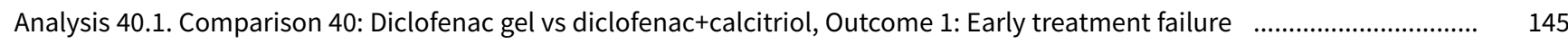

Analysis 41.1. Comparison 41: Calcitriol ointment vs calcitriol+diclofenac, Outcome 1: Early treatment failure ....................... 145

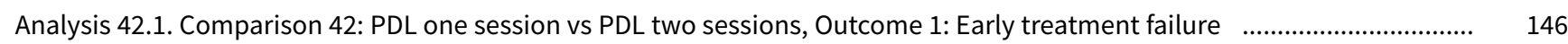

Analysis 43.1. Comparison 43: PDL 7mm spot-size single pulses vs PDL 10mm spot-size stacked pulses, Outcome 1: Early 146 treatment failure (lesions)

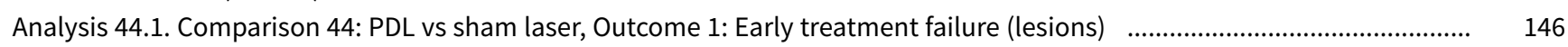

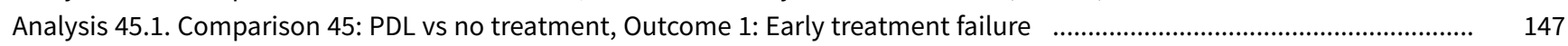

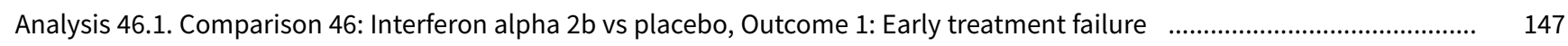

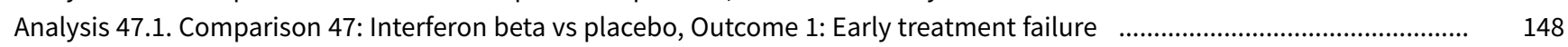

Analysis 48.1. Comparison 48: Interferon alpha 2a+2b vs interferon alpha 2a, Outcome 1: Early treatment failure $\quad . . . . . . . . . . . . . . . . . . \quad 148$

Analysis 49.1. Comparison 49: Interferon alpha 2a+2b vs interferon alpha 2b, Outcome 1: Early treatment failure $\ldots \ldots \ldots \ldots \ldots \ldots . . . . . . . . .148$

Analysis 50.1. Comparison 50: Interferon alpha 2b vs interferon alpha 2a, Outcome 1: Early treatment failure ..............................149

Analysis 51.1. Comparison 51: $3 x$ a week vs single dose protamine zinc chelate IFN alpha 2b, Outcome 1: Early treatment failure 149

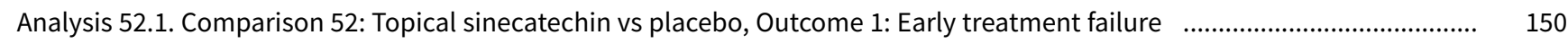

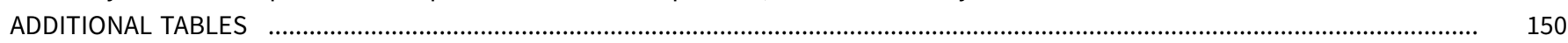

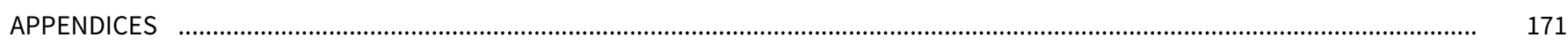

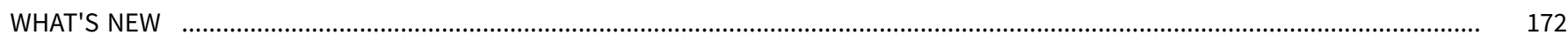

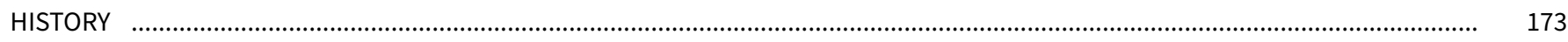

CONTRIBUTIONS OF AUTHORS

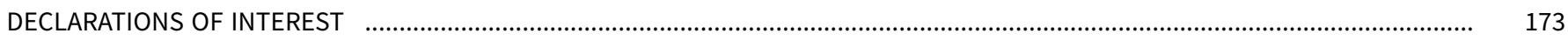

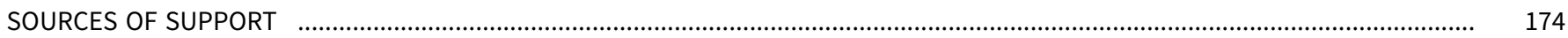

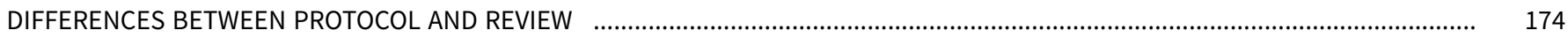

INDEX TERMS 
[Intervention Review]

\title{
Interventions for basal cell carcinoma of the skin
}

\author{
Jason Thomson ${ }^{1}$, Sarah Hogan ${ }^{1}$, Jo Leonardi-Bee ${ }^{2}$, Hywel C Williams 3 , Fiona J Bath-Hextall4
}

1Department of Dermatology, Royal London Hospital, Barts Health NHS Trust, London, UK. ${ }^{2}$ Centre for Evidence Based Healthcare, Division of Epidemiology and Public Health, Clinical Sciences Building Phase 2, University of Nottingham, Nottingham, UK. ${ }^{3}$ Centre of Evidence Based Dermatology, University of Nottingham, Nottingham, UK. ${ }^{4}$ Emeritus Professor, Evidence Based Health Care, University of Nottingham, Nottingham, UK

Contact address: Jason Thomson, Jason.thomson@nhs.net.

Editorial group: Cochrane Skin Group.

Publication status and date: New search for studies and content updated (conclusions changed), published in Issue 11, 2020.

Citation: Thomson J, Hogan S, Leonardi-Bee J, Williams HC, Bath-Hextall FJ. Interventions for basal cell carcinoma of the skin. Cochrane Database of Systematic Reviews 2020, Issue 11. Art. No.: CD003412. DOI: 10.1002/14651858.CD003412.pub3.

Copyright @ 2020 The Cochrane Collaboration. Published by John Wiley \& Sons, Ltd.

\section{A B S T R A C T}

\section{Background}

Basal cell carcinoma (BCC) is the commonest cancer affecting white-skinned individuals, and worldwide incidence is increasing. Although rarely fatal, BCC is associated with significant morbidity and costs. First-line treatment is usually surgical excision, but alternatives are available. New published studies and the development of non-surgical treatments meant an update of our Cochrane Review (first published in 2003, and previously updated in 2007) was timely.

\section{Objectives}

To assess the effects of interventions for BCC in immunocompetent adults.

\section{Search methods}

We updated our searches of the following databases to November 2019: Cochrane Skin Group Specialised Register, CENTRAL, MEDLINE, Embase, CINAHL, and LILACS.

\section{Selection criteria}

Randomised controlled trials (RCTs) of interventions for BCC in immunocompetent adults with histologically-proven, primary BCC. Eligible comparators were placebo, active treatment, other treatments, or no treatment.

\section{Data collection and analysis}

We used standard methodological procedures expected by Cochrane. Primary outcome measures were recurrence at three years and five years (measured clinically) (we included recurrence data outside of these time points if there was no measurement at three or five years) and participant- and observer-rated good/excellent cosmetic outcome. Secondary outcomes included pain during and after treatment, early treatment failure within six months, and adverse effects (AEs). We used GRADE to assess evidence certainty for each outcome.

\section{Main results}

We included 52 RCTs (26 new) involving 6690 participants (median 89) in this update. All studies recruited from secondary care outpatient clinics. More males than females were included. Study duration ranged from six weeks to 10 years (average 13 months). Most studies (48/52) included only low-risk BCC (superficial (sBCC) and nodular (nBCC) histological subtypes). The majority of studies were at low or unclear risk of bias for most domains. Twenty-two studies were industry-funded: commercial sponsors conducted most of the studies assessing imiquimod, and just under half of the photodynamic therapy (PDT) studies. 
Overall, surgical interventions have the lowest recurrence rates. For high-risk facial BCC (high-risk histological subtype or located in the facial 'H-zone' or both), there may be slightly fewer recurrences with Mohs micrographic surgery (MMS) compared to surgical excision (SE) at three years (1.9\% versus $2.9 \%$, respectively) (risk ratio (RR) $0.64,95 \%$ confidence interval (CI) 0.16 to $2.64 ; 1$ study, 331 participants; lowcertainty evidence) and at five years (3.2\% versus $5.2 \%$, respectively) (RR $0.61,95 \% \mathrm{Cl} 0.18$ to $2.04 ; 1$ study, 259 participants; low-certainty evidence). However, the $95 \% \mathrm{Cl}$ also includes the possibility of increased risk of recurrence and no difference between treatments. There may be little to no difference regarding improvement of cosmetic outcomes between MMS and SE, judged by participants and observers 18 months post-operatively (one study; low-certainty evidence); however, no raw data were available for this outcome.

When comparing imiquimod and SE for $\mathrm{nBCC}$ or SBCC at low-risk sites, imiquimod probably results in more recurrences than SE at three years (16.4\% versus $1.6 \%$, respectively) (RR $10.30,95 \% \mathrm{Cl} 3.22$ to $32.94 ; 1$ study, 401 participants; moderate-certainty evidence) and five years (17.5\% versus $2.3 \%$, respectively) (RR $7.73,95 \% \mathrm{Cl} 2.81$ to $21.3 ; 1$ study, 383 participants; moderate-certainty evidence). There may be little to no difference in the number of participant-rated good/excellent cosmetic outcomes (RR 1.00, 95\% Cl 0.94 to 1.06 ; 1 study, 326 participants; low-certainty evidence). However, imiquimod may result in greater numbers of good/excellent cosmetic outcomes compared to SE when observer-rated (60.6\% versus $35.6 \%$, respectively) (RR $1.70,95 \% \mathrm{Cl} 1.35$ to 2.15; 1 study, 344 participants; low-certainty evidence). Both cosmetic outcomes were measured at three years.

Based on one study of 347 participants with high- and low-risk primary BCC of the face, radiotherapy may result in more recurrences compared to SE under frozen section margin control at three years (5.2\% versus $0 \%$, respectively) (RR $19.11,95 \% \mathrm{Cl} 1.12$ to 325.78 ; lowcertainty evidence) and at four years (6.4\% versus $0.6 \%$, respectively) (RR $11.06,95 \% \mathrm{Cl} 1.44$ to 84.77 ; low-certainty evidence). Radiotherapy probably results in a smaller number of good participant- (RR $0.76,95 \% \mathrm{Cl} 0.63$ to $0.91 ; 50.3 \%$ versus $66.1 \%$, respectively) or observer-rated (RR $0.48,95 \% \mathrm{Cl} 0.37$ to $0.62 ; 28.9 \%$ versus $60.3 \%$, respectively) good/excellent cosmetic outcomes compared to SE, when measured at four years, where dyspigmentation and telangiectasia can occur (both moderate-certainty evidence).

Methyl-aminolevulinate (MAL)-PDT may result in more recurrences compared to SE at three years (36.4\% versus $0 \%$, respectively) (RR $26.47,95 \% \mathrm{Cl} 1.63$ to $429.92 ; 1$ study; 68 participants with low-risk nBCC in the head and neck area; low-certainty evidence). There were no useable data for measurement at five years. MAL-PDT probably results in greater numbers of participant- $(\mathrm{RR} 1.18,95 \% \mathrm{Cl} 1.09$ to 1.27 ; $97.3 \%$ versus $82.5 \%$ ) or observer-rated (RR $1.87,95 \% \mathrm{Cl} 1.54$ to $2.26 ; 87.1 \%$ versus $46.6 \%$ ) good/excellent cosmetic outcomes at one year compared to SE (2 studies, 309 participants with low-risk nBCC and SBCC; moderate-certainty evidence).

Based on moderate-certainty evidence (single low-risk SBCC), imiquimod probably results in fewer recurrences at three years compared to MAL-PDT ( $22.8 \%$ versus $51.6 \%$, respectively) (RR $0.44,95 \% \mathrm{Cl} 0.32$ to $0.62 ; 277$ participants) and five years ( $28.6 \%$ versus $68.6 \%$, respectively) (RR $0.42,95 \% \mathrm{Cl} 0.31$ to $0.57 ; 228$ participants). There is probably little to no difference in numbers of observer-rated good/excellent cosmetic outcomes at one year (RR $0.98,95 \% \mathrm{Cl} 0.84$ to $1.16 ; 370$ participants). Participant-rated cosmetic outcomes were not measured for this comparison.

AEs with surgical interventions include wound infections, graft necrosis and post-operative bleeding. Local AEs such as itching, weeping, pain and redness occur frequently with non-surgical interventions. Treatment-related AEs resulting in study modification or withdrawal occurred with imiquimod and MAL-PDT.

\section{Authors' conclusions}

Surgical interventions have the lowest recurrence rates, and there may be slightly fewer recurrences with MMS over SE for high-risk facial primary BCC (low-certainty evidence). Non-surgical treatments, when used for low-risk BCC, are less effective than surgical treatments, but recurrence rates are acceptable and cosmetic outcomes are probably superior. Of the non-surgical treatments, imiquimod has the best evidence to support its efficacy.

Overall, evidence certainty was low to moderate. Priorities for future research include core outcome measures and studies with longerterm follow-up.

\section{PLAIN LANGUAGE SUMMARY}

\section{Interventions for basal cell carcinoma of the skin}

\section{Why is this question important?}

Basal cell carcinoma (BCC) is the most common form of skin cancer among people with white skin.

BCC is not usually life-threatening but if left untreated, it can cause important disfigurement, especially on the face.

Surgical removal of the affected area and surrounding skin is usually the first option for treating BCC. Several different surgical approaches exist as well as non-surgical treatments, such as radiotherapy (high doses of radiation that kill cancer cells), and anti-cancer creams, gels and ointments.

We reviewed the evidence from research studies, to find out which treatments work best for BCC. 


\section{How did we identify and evaluate the evidence?}

We searched for randomised controlled studies (studies where people are randomly put into one of two or more treatment groups), because these provide the most robust evidence about the effects of a treatment. We compared and summarised the evidence from all the studies. Finally, we rated our confidence in the evidence, based on factors such as study methods and sizes, and the consistency of findings across studies.

\section{What did we find?}

We found 52 studies that involved a total of 6690 adults with BCC. Most studies (48 out of 52) included hospital outpatients with small, superficial or nodular BCC. Studies lasted for between six weeks and 10 years (average duration: 13 months). Twenty-two studies were funded by pharmaceutical companies.

Our confidence in the evidence presented here is low to moderate, mainly because many studies were small.

\section{Comparison between different surgical treatments}

- Mohs micrographic surgery (a specialised surgical approach that removes less skin) may slightly decrease recurrence rates at three and five years compared to surgical excision (one of the most common surgical treatments for BCC).

-There may be little to no difference in how well scars heal between these two surgical treatments according to patients and observers (one study).

\section{Surgery versus non-surgical treatments}

Compared against surgical excision:

- Imiquimod (an anti-cancer cream) probably increases BCC recurrence rates at three and five years. There may be little to no difference in scar healing according to patients, although imiquimod may increase chances of scars healing well when healing is rated by an observer (one study).

- Radiotherapy may increase BCC recurrence rates at three and four years, and probably decreases chances of scars healing well (one study). - MAL-PDT, a type of photodynamic therapy (that uses a light source and light-sensitive medicine to kill cancer cells), may increase BCC recurrence rates at three years. MAL-PDT probably increases chances of scars healing well (two studies).

\section{Comparison of different non-surgical treatments}

Compared against imiquimod:

- MAL-PDT probably increases BCC recurrence rates at three and five years. There is probably little to no difference in scar healing (one study);

\section{What does this mean?}

Overall, the evidence suggests that:

- surgery could reduce chances of BCC recurrence;

- non-surgical treatments such as anti-cancer creams or photodynamic therapy carry an increased chance of BCC recurrence, but could increase chances of scars healing well compared with surgery.

Complications with surgical treatments include wound infections, skin graft failure and bleeding after the procedure. Non-surgical treatments frequently lead to localised itching, weeping, pain and redness. Treatment-related side effects that caused modifications to the study or the withdrawal of participants occurred with imiquimod and MAL-PDT.

\section{How-up-to date is this review?}

The evidence in this Cochrane Review is current to November 2019. 


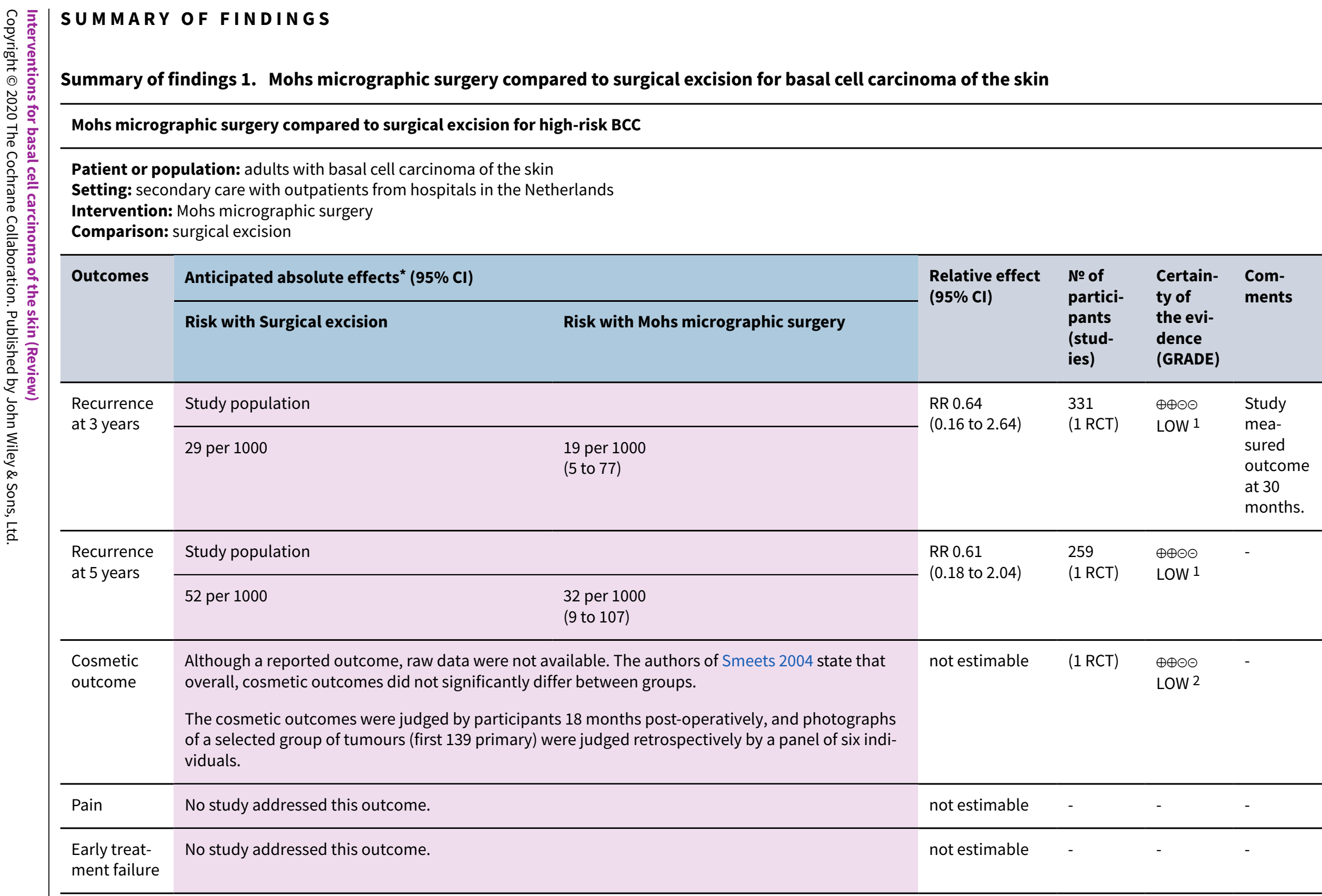

*The risk in the intervention group (and its $95 \%$ confidence interval) is based on the assumed risk in the comparison group and the relative effect of the intervention (and its $95 \% \mathrm{Cl}$ ). 


\section{GRADE Working Group grades of evidence}

High certainty: we are very confident that the true effect lies close to that of the estimate of the effect.

Moderate certainty: we are moderately confident in the effect estimate; the true effect is likely to be close to the estimate of the effect, but there is a possibility that it is

substantially different.

Low certainty: our confidence in the effect estimate is limited; the true effect may be substantially different from the estimate of the effect.

Very low certainty: we have very little confidence in the effect estimate; the true effect is likely to be substantially different from the estimate of effect.

1 Downgraded two levels for very serious imprecision as very wide $95 \% \mathrm{Cl}$ indicating the possibility of important benefit or harm.

2 Downgraded two levels for very serious indirectness as although the authors did compare the cosmetic outcomes between the two groups, they did not present the data for analysis.

\section{Summary of findings 2. Imiquimod compared to surgical excision for basal cell carcinoma of the skin}

\section{Imiquimod compared to surgical excision for low-risk BCC}

Patient or population: adults with basal cell carcinoma of the skin Setting: secondary care with outpatients from hospitals in the UK

Intervention: 5\% imiquimod cream

Comparison: surgical excision

\begin{tabular}{|c|c|c|c|c|c|c|}
\hline \multirow[t]{2}{*}{ Outcomes } & \multicolumn{2}{|c|}{ Anticipated absolute effects ${ }^{\star}(95 \% \mathrm{Cl})$} & \multirow{2}{*}{$\begin{array}{l}\text { Relative effect } \\
(95 \% \mathrm{CI})\end{array}$} & \multirow{2}{*}{$\begin{array}{l}\text { № of par- } \\
\text { ticipants } \\
\text { (studies) }\end{array}$} & \multirow{2}{*}{$\begin{array}{l}\text { Certainty } \\
\text { of the evi- } \\
\text { dence } \\
\text { (GRADE) }\end{array}$} & \multirow[t]{2}{*}{ Comments } \\
\hline & $\begin{array}{l}\text { Risk with surgical exci- } \\
\text { sion }\end{array}$ & Risk with Imiquimod & & & & \\
\hline \multirow[t]{2}{*}{ Recurrence at 3 years } & \multicolumn{2}{|l|}{ Study population } & \multirow{2}{*}{$\begin{array}{l}\text { RR } 10.30 \\
\text { (3.22 to } 32.94)\end{array}$} & \multirow{2}{*}{$\begin{array}{l}401 \\
(1 \mathrm{RCT})\end{array}$} & \multirow{2}{*}{$\begin{array}{l}\oplus \oplus \oplus \ominus \\
\text { MODERATE } \\
1\end{array}$} & \multirow[t]{2}{*}{-} \\
\hline & 16 per 1000 & $\begin{array}{l}164 \text { per } 1000 \\
\text { (51 to } 526)\end{array}$ & & & & \\
\hline \multirow[t]{2}{*}{ Recurrence at 5 years } & \multicolumn{2}{|l|}{ Study population } & \multirow{2}{*}{$\begin{array}{l}\text { RR } 7.73 \\
\text { (2.81 to } 21.30 \text { ) }\end{array}$} & \multirow{2}{*}{$\begin{array}{l}383 \\
(1 \mathrm{RCT})\end{array}$} & \multirow{2}{*}{$\begin{array}{l}\oplus \oplus \oplus \ominus \\
\text { MODERATE } \\
1\end{array}$} & \multirow[t]{2}{*}{-} \\
\hline & 23 per 1000 & $\begin{array}{l}175 \text { per } 1000 \\
\text { (64 to } 481)\end{array}$ & & & & \\
\hline \multirow{2}{*}{$\begin{array}{l}\text { Cosmetic outcome } \\
\text { (good/excellent) }\end{array}$} & \multicolumn{2}{|l|}{ Study population } & \multirow{2}{*}{$\begin{array}{l}\text { RR } 1.70 \\
\text { (1.35 to } 2.15 \text { ) }\end{array}$} & \multirow{2}{*}{$\begin{array}{l}344 \\
(1 \mathrm{RCT})\end{array}$} & \multirow{2}{*}{$\begin{array}{l}\oplus \oplus \Theta \Theta \\
\text { LOW } 2\end{array}$} & \multirow{2}{*}{$\begin{array}{l}\text { Observer-rated at } 3 \\
\text { years on a } 6 \text {-point } \\
\text { scale. }{ }^{4}\end{array}$} \\
\hline & 356 per 1000 & $\begin{array}{l}606 \text { per } 1000 \\
\text { (481 to } 766)\end{array}$ & & & & \\
\hline
\end{tabular}




\begin{tabular}{|c|c|c|c|c|c|c|}
\hline \multirow{2}{*}{ 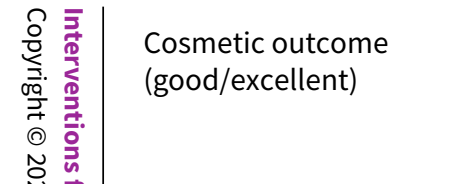 } & \multicolumn{2}{|c|}{ Study population } & \multirow{2}{*}{$\begin{array}{l}\text { RR } 1.00 \\
\text { (0.94 to } 1.06)\end{array}$} & \multirow{2}{*}{$\begin{array}{l}326 \\
(1 \mathrm{RCT})\end{array}$} & \multirow{2}{*}{$\begin{array}{l}\oplus \oplus \ominus \ominus \\
\text { LOW } 2\end{array}$} & \multirow{2}{*}{$\begin{array}{l}\text { Participant-rated at } \\
3 \text { years on a } 6 \text {-point } \\
\text { scale. }^{4}\end{array}$} \\
\hline & 922 per 1000 & $\begin{array}{l}922 \text { per } 1000 \\
\text { (866 to } 977)\end{array}$ & & & & \\
\hline \multirow[t]{2}{*}{ Pain (moderate/severe) } & \multicolumn{2}{|c|}{ Study population } & \multirow{2}{*}{$\begin{array}{l}\text { RR } 1.36 \\
\text { (0.98 to } 1.88 \text { ) }\end{array}$} & \multirow{2}{*}{$\begin{array}{l}443 \\
(1 \mathrm{RCT})\end{array}$} & \multirow{2}{*}{$\begin{array}{l}\oplus \oplus \odot \odot \\
\text { LOW } 3\end{array}$} & \multirow{2}{*}{$\begin{array}{l}\text { Pain measured during } \\
\text { treatment. } 5\end{array}$} \\
\hline & 219 per 1000 & $\begin{array}{l}298 \text { per } 1000 \\
\text { (215 to } 412)\end{array}$ & & & & \\
\hline \multirow[t]{2}{*}{ Pain (moderate/severe) } & \multicolumn{2}{|c|}{ Study population } & \multirow{2}{*}{$\begin{array}{l}\text { RR } 0.47 \\
(0.29 \text { to } 0.77)\end{array}$} & \multirow{2}{*}{$\begin{array}{l}439 \\
(1 \mathrm{RCT})\end{array}$} & \multirow{2}{*}{$\begin{array}{l}\oplus \oplus \odot \odot \\
\text { LOW }^{3}\end{array}$} & \multirow{2}{*}{$\begin{array}{l}\text { Pain measured during } \\
\text { follow-up. } 5\end{array}$} \\
\hline & 199 per 1000 & $\begin{array}{l}94 \text { per } 1000 \\
\text { (58 to } 153)\end{array}$ & & & & \\
\hline Early treatment failure & \multicolumn{2}{|c|}{ No study addressed this outcome } & not estimable & - & - & - \\
\hline
\end{tabular}

${ }^{\star}$ The risk in the intervention group (and its $95 \%$ confidence interval) is based on the assumed risk in the comparison group and the relative effect of the intervention (and its $95 \% \mathrm{Cl})$.

Cl: Confidence interval; RR: Risk ratio.

\section{GRADE Working Group grades of evidence}

High certainty: we are very confident that the true effect lies close to that of the estimate of the effect.

Moderate certainty: we are moderately confident in the effect estimate; the true effect is likely to be close to the estimate of the effect, but there is a possibility that it is substantially different.

Low certainty: our confidence in the effect estimate is limited; the true effect may be substantially different from the estimate of the effect.

Very low certainty: we have very little confidence in the effect estimate; the true effect is likely to be substantially different from the estimate of effect.

1 Downgraded one level for serious imprecision as only a single study with a small sample size and a wide $95 \% \mathrm{Cl}$.

2 Downgraded one level for serious imprecision as only a single study with a small sample size and one level for serious risk of bias as unable to truly blind due to the nature of interventions (i.e. presence or absence of scar will unblind to treatment allocation).

3 Downgraded one level for serious imprecision as only a single study with a small sample size and one level for serious risk of attrition bias as fewer pain data were available for the surgical excision group.

4 6-point scale: unable to see lesion, very poor, poor, fair, good, excellent.

5 Measured on a scale from: no pain, mild pain, mild-to-moderate pain, moderate pain, moderate-to-severe pain and severe pain

\section{Summary of findings 3. Radiotherapy compared to surgical excision (with or without frozen section margin control) for basal cell carcinoma of the} skin

Radiotherapy compared to surgical excision for high- and low-risk BCC 
Patient or population: adults with basal cell carcinoma of the skin

Setting: secondary care with outpatients from a single hospital in France

Intervention: radiotherapy

Comparison: surgical excision (with or without frozen section margin control)

\begin{tabular}{|c|c|c|c|c|c|c|}
\hline \multirow[t]{2}{*}{ Outcomes } & \multicolumn{2}{|c|}{ Anticipated absolute effects ${ }^{\star}(95 \% \mathrm{Cl})$} & \multirow{2}{*}{$\begin{array}{l}\text { Relative effect } \\
(95 \% \mathrm{CI})\end{array}$} & \multirow{2}{*}{$\begin{array}{l}\text { № of par- } \\
\text { ticipants } \\
\text { (studies) }\end{array}$} & \multirow{2}{*}{$\begin{array}{l}\text { Certainty } \\
\text { of the evi- } \\
\text { dence } \\
\text { (GRADE) }\end{array}$} & \multirow[t]{2}{*}{ Comments } \\
\hline & $\begin{array}{l}\text { Risk with surgical exci- } \\
\text { sion }\end{array}$ & Risk with radiotherapy & & & & \\
\hline \multirow[t]{2}{*}{ Recurrence at 3 years } & \multicolumn{2}{|l|}{ Study population } & \multirow{2}{*}{$\begin{array}{l}\text { RR } 19.11 \\
\text { (1.12 to } 325.78)\end{array}$} & \multirow{2}{*}{$\begin{array}{l}347 \\
(1 \mathrm{RCT})\end{array}$} & \multirow{2}{*}{$\begin{array}{l}\oplus \oplus \ominus \ominus \\
\text { LOW } 1\end{array}$} & \multirow[t]{2}{*}{-} \\
\hline & 0 per 1000 & $\begin{array}{l}52 \text { per } 1000 \\
\text { (6 to } 1883)\end{array}$ & & & & \\
\hline \multirow[t]{2}{*}{ Recurrence at 4 years } & \multicolumn{2}{|l|}{ Study population } & \multirow{2}{*}{$\begin{array}{l}\text { RR } 11.06 \\
\text { (1.44 to } 84.77 \text { ) }\end{array}$} & \multirow{2}{*}{$\begin{array}{l}347 \\
(1 \mathrm{RCT})\end{array}$} & \multirow{2}{*}{$\begin{array}{l}\oplus \oplus \odot \odot \\
\text { LOW } 2\end{array}$} & \multirow[t]{2}{*}{-} \\
\hline & 6 per 1000 & $\begin{array}{l}64 \text { per } 1000 \\
\text { ( } 8 \text { to } 487)\end{array}$ & & & & \\
\hline \multirow{2}{*}{$\begin{array}{l}\text { Cosmetic outcome } \\
\text { (good/excellent) }\end{array}$} & \multicolumn{2}{|l|}{ Study population } & \multirow[t]{2}{*}{ RR 0.76 ( 0.63 to 0.91$)$} & \multirow{2}{*}{$\begin{array}{l}347 \\
(1 \mathrm{RCT})\end{array}$} & \multirow{2}{*}{$\begin{array}{l}\oplus \oplus \oplus \odot \\
\text { MODERATE }\end{array}$} & \multirow{2}{*}{$\begin{array}{l}\text { Participant-rated at } \\
4 \text { years on a 3-point } \\
\text { scale. } 4 \text { ITT analysis per- } \\
\text { formed. }\end{array}$} \\
\hline & 661 per 1000 & $\begin{array}{l}502 \text { per } 1000 \\
(416 \text { to } 601)\end{array}$ & & & & \\
\hline \multirow{2}{*}{$\begin{array}{l}\text { Cosmetic outcome } \\
\text { (good/excellent) }\end{array}$} & \multicolumn{2}{|l|}{ Study population } & \multirow{2}{*}{$\begin{array}{l}\text { RR } 0.48 \\
(0.37 \text { to } 0.62)\end{array}$} & \multirow{2}{*}{$\begin{array}{l}347 \\
(1 \mathrm{RCT})\end{array}$} & \multirow{2}{*}{$\begin{array}{l}\oplus \oplus \oplus \ominus \\
\text { MODERATE } 3\end{array}$} & \multirow{2}{*}{$\begin{array}{l}\text { Observer-rated at } 4 \\
\text { years on a } 3 \text {-point } \\
\text { scale. }{ }^{4} \text { ITT analysis per- } \\
\text { formed. }\end{array}$} \\
\hline & 603 per 1000 & $\begin{array}{l}290 \text { per } 1000 \\
(223 \text { to } 374)\end{array}$ & & & & \\
\hline Pain & \multicolumn{2}{|c|}{ No study addressed this outcome. } & not estimable & - & - & - \\
\hline Early treatment failure & \multicolumn{2}{|c|}{ No study addressed this outcome. } & not estimable & - & - & - \\
\hline
\end{tabular}

*The risk in the intervention group (and its $95 \%$ confidence interval) is based on the assumed risk in the comparison group and the relative effect of the intervention (and its $95 \% \mathrm{Cl}$ ).

Cl: Confidence interval; ITT: intention-to-treat; RR: Risk ratio.

\section{GRADE Working Group grades of evidence}

High certainty: we are very confident that the true effect lies close to that of the estimate of the effect.

Moderate certainty: we are moderately confident in the effect estimate; the true effect is likely to be close to the estimate of the effect, but there is a possibility that it is substantially different.

Low certainty: our confidence in the effect estimate is limited; the true effect may be substantially different from the estimate of the effect. 


\begin{tabular}{|c|c|c|c|c|c|c|}
\hline \multicolumn{7}{|c|}{$\begin{array}{l}1 \text { Downgraded two levels for very serious imprecision due to very wide } 95 \% \mathrm{Cl} \text { (although excludes } 1 \text {, there is a greater than } 100 \text {-fold differen } \\
2 \text { Downgraded one level for serious indirectness (outcome outside our pre-specified time-points) and downgraded one level for serious im, } \\
\text { a small sample size. } \\
3 \text { Downgraded one level for serious risk of bias as unable to truly blind due to the nature of interventions. } \\
4 \text { 3-point scale: bad, fair, good. }\end{array}$} \\
\hline \multicolumn{7}{|c|}{ Summary of findings 4. MAL-PDT compared to surgical excision for basal cell carcinoma of the skin } \\
\hline \multicolumn{7}{|c|}{ MAL-PDT compared to surgical excision for low-risk BCC } \\
\hline \multicolumn{7}{|c|}{$\begin{array}{l}\text { Patient or population: adults with basal cell carcinoma of the skin } \\
\text { Setting: secondary care with outpatients from hospitals in Brazil, the UK, Germany, Switzerland and Australia } \\
\text { Intervention: MAL-PDT } \\
\text { Comparison: surgical excision }\end{array}$} \\
\hline \multirow[t]{2}{*}{ Outcomes } & \multicolumn{2}{|c|}{ Anticipated absolute effects ${ }^{*}(95 \% \mathrm{Cl})$} & \multirow{2}{*}{$\begin{array}{l}\text { Relative effect } \\
(95 \% \mathrm{CI})\end{array}$} & \multirow{2}{*}{$\begin{array}{l}\text { No of partic- } \\
\text { ipants } \\
\text { (studies) }\end{array}$} & \multirow{2}{*}{$\begin{array}{l}\text { Certainty of } \\
\text { the evidence } \\
\text { (GRADE) }\end{array}$} & \multirow[t]{2}{*}{ Comments } \\
\hline & $\begin{array}{l}\text { Risk with surgical ex- } \\
\text { cision }\end{array}$ & Risk with MAL-PDT & & & & \\
\hline \multirow{2}{*}{$\begin{array}{l}\text { Recurrence at } 3 \\
\text { years }\end{array}$} & Study population & & \multirow{2}{*}{$\begin{array}{l}\text { RR } 26.47 \\
\text { (1.63 to } 429.92)\end{array}$} & \multirow{2}{*}{$\begin{array}{l}68 \\
(1 \mathrm{RCT})\end{array}$} & \multirow{2}{*}{$\begin{array}{l}\oplus \oplus \ominus \ominus \\
\text { LOW } 1\end{array}$} & \multirow[t]{2}{*}{-} \\
\hline & 0 per 1000 & $\begin{array}{l}364 \text { per } 1000 \\
\text { (49 to } 13027)\end{array}$ & & & & \\
\hline \multirow{2}{*}{$\begin{array}{l}\text { Cosmetic outcome } \\
\text { (excellent/good) }\end{array}$} & Study population & & \multirow{2}{*}{$\begin{array}{l}\text { RR } 1.18 \\
\text { (1.09 to } 1.27 \text { ) }\end{array}$} & \multirow{2}{*}{$\begin{array}{l}309 \\
(2 \text { RCTs) }\end{array}$} & \multirow{2}{*}{$\begin{array}{l}\oplus \oplus \oplus \odot \\
\text { MODERATE } 2\end{array}$} & \multirow{2}{*}{$\begin{array}{l}\text { Participant-rated at } 1 \text { year on a } \\
\text { 4-point scale. } 5\end{array}$} \\
\hline & 825 per 1000 & $\begin{array}{l}973 \text { per } 1000 \\
\text { (899 to } 1000)\end{array}$ & & & & \\
\hline \multirow{2}{*}{$\begin{array}{l}\text { Cosmetic outcome } \\
\text { (excellent/good) }\end{array}$} & Study population & & \multirow{2}{*}{$\begin{array}{l}\text { RR } 1.87 \\
\text { (1.54 to } 2.26 \text { ) }\end{array}$} & \multirow{2}{*}{$\begin{array}{l}256 \\
(2 \mathrm{RCTs})\end{array}$} & \multirow{2}{*}{$\begin{array}{l}\oplus \oplus \oplus \odot \\
\text { MODERATE } 2\end{array}$} & \multirow{2}{*}{$\begin{array}{l}\text { Observer-rated at } 1 \text { year on a } 4 \text { - } \\
\text { point scale. } 5\end{array}$} \\
\hline & 466 per 1000 & $\begin{array}{l}871 \text { per } 1000 \\
\text { (717 to } 1000)\end{array}$ & & & & \\
\hline \multirow[t]{2}{*}{ Pain } & Study population & & \multirow{2}{*}{$\begin{array}{l}\text { RR } 2.20 \\
\text { (0.60 to } 8.03)\end{array}$} & \multirow{2}{*}{$\begin{array}{l}101 \\
(1 \mathrm{RCT})\end{array}$} & \multirow{2}{*}{$\begin{array}{l}\oplus \oplus \odot \odot \\
\text { LOW }\end{array}$} & \multirow{2}{*}{$\begin{array}{l}\text { Study reported frequency of } \\
\text { "pain in skin" and "burning sen- } \\
\text { sation of skin" as part of AEs. }\end{array}$} \\
\hline & 61 per 1000 & $\begin{array}{l}135 \text { per } 1000 \\
\text { (37 to } 492)\end{array}$ & & & & \\
\hline
\end{tabular}




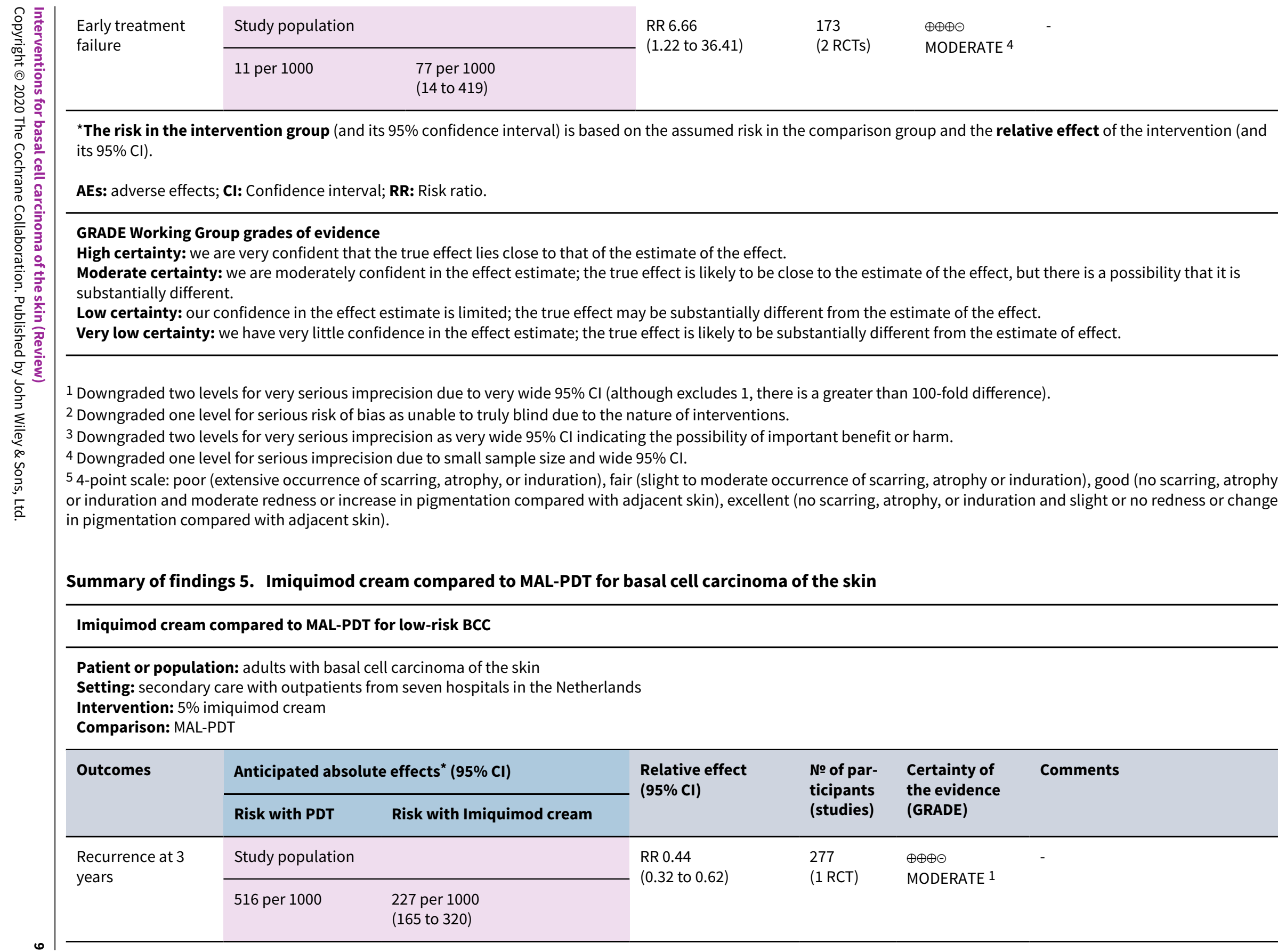




\begin{tabular}{|c|c|c|c|c|c|c|c|}
\hline \multirow{2}{*}{\multicolumn{2}{|c|}{ 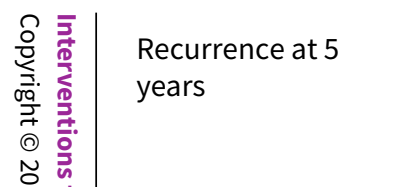 }} & \multicolumn{2}{|c|}{ Study population } & \multirow{2}{*}{$\begin{array}{l}\text { RR } 0.42 \\
(0.31 \text { to } 0.57)\end{array}$} & \multirow{2}{*}{$\begin{array}{l}228 \\
(1 \mathrm{RCT})\end{array}$} & \multirow{2}{*}{$\begin{array}{l}\oplus \oplus \oplus \ominus \\
\text { MODERATE } 1\end{array}$} & \multirow[t]{2}{*}{-} \\
\hline & & 686 per 1000 & $\begin{array}{l}288 \text { per } 1000 \\
(213 \text { to } 391)\end{array}$ & & & & \\
\hline 青 & \multirow{2}{*}{$\begin{array}{l}\text { Cosmetic outcome } \\
\text { (excellent/good) }\end{array}$} & \multicolumn{2}{|c|}{ Study population } & \multirow{2}{*}{$\begin{array}{l}\text { RR } 0.98 \\
\text { (0.84 to } 1.16)\end{array}$} & \multirow{2}{*}{$\begin{array}{l}370 \\
(1 \mathrm{RCT})\end{array}$} & \multirow{2}{*}{$\begin{array}{l}\oplus \oplus \oplus \ominus \\
\text { MODERATE } 1\end{array}$} & \multirow{2}{*}{$\begin{array}{l}\text { Blinded observer-rated at } 1 \text { year } \\
\text { on } 4 \text {-point scale. } 3\end{array}$} \\
\hline 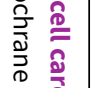 & & 624 per 1000 & $\begin{array}{l}611 \text { per } 1000 \\
\text { (524 to } 723)\end{array}$ & & & & \\
\hline 咅 0 & \multirow{2}{*}{$\begin{array}{l}\text { Pain (moderate/se- } \\
\text { vere) }\end{array}$} & \multicolumn{2}{|c|}{ Study population } & \multirow{2}{*}{$\begin{array}{l}\text { RR } 0.60 \\
\text { (0.41 to } 0.87)\end{array}$} & \multirow{2}{*}{$\begin{array}{l}371 \\
(1 \mathrm{RCT})\end{array}$} & \multirow{2}{*}{$\begin{array}{l}\oplus \oplus \oplus \ominus \\
\text { MODERATE } 1\end{array}$} & \multirow{2}{*}{$\begin{array}{l}\text { During treatment: week of treat- } \\
\text { ment with highest frequency of } \\
\text { reported moderate/severe pain } \\
\text { (week } 6 \text { for imiquimod, treat- } \\
\text { ment cycle } 2 \text { for PDT). }\end{array}$} \\
\hline 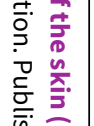 & & 305 per 1000 & $\begin{array}{l}183 \text { per } 1000 \\
(125 \text { to } 266)\end{array}$ & & & & \\
\hline 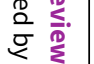 & \multirow{2}{*}{$\begin{array}{l}\text { Early treatment } \\
\text { failure }\end{array}$} & Study popula & & \multirow{2}{*}{$\begin{array}{l}\text { RR } 0.64 \\
\text { (0.37 to } 1.09)\end{array}$} & \multirow{2}{*}{$\begin{array}{l}385 \\
(1 \mathrm{RCT})\end{array}$} & \multirow{2}{*}{$\begin{array}{l}\oplus \oplus \oplus \odot \\
\text { MODERATE } 2\end{array}$} & \multirow[t]{2}{*}{-} \\
\hline$\sum_{\leqq}^{\bar{J}}$ & & 158 per 1000 & $\begin{array}{l}101 \text { per } 1000 \\
\text { (59 to } 172)\end{array}$ & & & & \\
\hline
\end{tabular}

${ }^{\star}$ The risk in the intervention group (and its $95 \%$ confidence interval) is based on the assumed risk in the comparison group and the relative effect of the intervention (and its $95 \% \mathrm{Cl})$.

Cl: Confidence interval; RR: Risk ratio.

\section{GRADE Working Group grades of evidence}

High certainty: we are very confident that the true effect lies close to that of the estimate of the effect.

Moderate certainty: we are moderately confident in the effect estimate; the true effect is likely to be close to the estimate of the effect, but there is a possibility that it is substantially different.

Low certainty: our confidence in the effect estimate is limited; the true effect may be substantially different from the estimate of the effect.

Very low certainty: we have very little confidence in the effect estimate; the true effect is likely to be substantially different from the estimate of effect.

1 Downgraded one level for serious imprecision as only a single study with a small sample size.

2 Downgraded one level for serious imprecision as only a single study with a small sample size and a wide $95 \% \mathrm{Cl}$.

3 4-point scale: poor, fair, good, excellent. 


\section{B A C K G R O U N D}

\section{Description of the condition}

\section{Disease definition and burden}

Basal cell carcinoma (BCC) is the most common skin cancer and the most common cancer found in white-skinned individuals (Dessinioti 2010; Lomas 2012; Madan 2016; Verkouteren 2017). BCCs are slow-growing, locally invasive, malignant (but not life threatening), epidermal skin tumours (Roewert-Huber 2007; Wong 2003). Patients with BCC place a considerable burden on healthcare systems worldwide due to their high, increasing incidence and associated morbidity. Furthermore, people with $\mathrm{BCC}$ are at high risk of developing further BCCs and other ultraviolet radiation (UVR)related skin cancers. As a result, the disability adjusted life years and healthcare costs of BCCs have risen significantly in recent decades (Verkouteren 2017).

\section{Clinical features}

Clinical appearances and morphology of BCC can be diverse (more than 26 subtypes have been described), with no universally accepted classification system. The main clinical subtypes are: nodular, superficial, ulcerated (rodent ulcer), morphoeic (sclerodermiform), fibroepithelial (fibroepithelioma of Pinkus) and advanced BCC (resulting from prolonged neglect) (Madan 2016). Histopathological patterns are also diverse and include: nodular superficial, morphoeic, micronodular, infiltrative, pigmented and basosquamous or metatypical subtypes (Crowson 2006). Early BCCs may appear as a small red patch or a scab that fails to heal. Symptoms such as pain are rare. Nodular BCC (nBCC) is the most common subtype in the UK, making up to $60 \%$ of all BCCs (Scrivener 2002). However, in other countries such as Australia, superficial BCC (sBCC) is the most common subtype (Raasch 2002). BCCs affect the head and neck region in the majority of cases (Scrivener 2002). A recent UK study of first registered BCCs in the population identified BCCs affected the head and neck region around $70 \%$ of the time and the trunk and extremities around $30 \%$ of the time (Venables 2019).

Clinicopathological features of the tumours can be used to differentiate them into high-risk and low-risk subtypes, which has implications on the management approach. High-risk BCCs include: morphoeic, infiltrative, micronodular histological subtypes; presence of perineural or perivascular invasion; size $>5$ $\mathrm{cm}$; recurrent lesion; centrofacial location, including periocular and ears and host immunosuppression (Madan 2016). Low-risk BCCs include: superficial and nodular histological subtypes when they are located on a low-risk site (e.g. not centrofacial location).

\section{Natural history}

BCCs are usually slow-growing tumours that only very rarely metastasize (spread) to other distant parts of the body $(0.0028 \%$ to $0.55 \%$ of advanced BCCs metastasize) (Ting 2005). In people with a competent immune system, growth of a BCC is usually localised to the area of origin. BCCs tend to infiltrate surrounding tissues in a three-dimensional fashion through the irregular growth of fingerlike projections which may not be apparent clinically (Breuninger 1991; Miller 1991). If left untreated, or inadequately treated, the $\mathrm{BCC}$ can cause extensive tissue destruction, particularly on the face. Neglected cases may even infiltrate bone and deeper structures, like the brain. The clinical course of BCC is unpredictable; it may remain small for years with little tendency to grow, it may grow rapidly, or it may proceed by successive spurts of extension of tumour and partial regression (Franchimont 1982).

\section{Epidemiology and causes}

Considerable variation exists in the literature for incidence rates of BCC. Latitude, sun exposure and skin type of a country's inhabitants contribute to much of this variation. Additionally, despite being common tumours, numbers of BCCs recorded in cancer registries are frequently underestimated due to their high volume and low perceived risk and often they are treated without a confirmatory biopsy. Depending on the registry, it is estimated that between $30 \%$ to $50 \%$ of BCCs are unreported (NCIN 2010). However, since 2013 the UK has vastly improved registry data collection and now records all cases of BCC and squamous cell carcinoma (SCC). This has greatly improved the accuracy of epidemiological analysis of keratinocyte cancers and the UK now has the largest populationbased keratinocyte cancer registry in the world (Venables 2019). From 2013 to 2015, the European age standardised incidence rate of BCC in the UK was 285 per 100,000 people annually. The highest $B C C$ rate was observed in Southwest England with 362 per 100,000 people annually.

Direct comparison of incidence rates between countries is limited by the fact that studies often use different standardisation methods and because each country will have their own registry with different registration criteria. The highest incidence of BCC is found in Australia where in Queensland from 1997 to 2006, the rate was 1813 per 100,000 males annually and 1269 per 100,000 females annually (Verkouteren 2017). Parts of Africa have the lowest reported incidence rates with less than 1 per 100,000 people annually (Lomas 2012). In mainland Europe, there is large variation dependent on country and sex with the highest rate of 164.7 per 100,000 males annually found in the Netherlands and the lowest rate of 24.5 per 100,000 females annually observed in Croatia (Verkouteren 2017). In the USA, California had the highest incidence rate of 1069 per 100,000 males annually. Data from the USA provides compelling evidence for the role of decreasing geographical latitude on increasing BCC incidence with a rate in New Hampshire (43 degrees) of 309.9 per 100,000 males annually (Karagas 1999) whereas in Arizona (35 degrees) the rate is 935.9 per 100,000 males annually (Harris 2001). The studies reporting these incidence rates both used the same standardisation method.

The incidence of BCC is increasing globally. A systematic review identified that incidence is increasing in Europe by $5.5 \%$ annually (Lomas 2012), and the study by Venables 2019 calculated a mean annual percentage increase of 5\% between 2013 to 2015 for both BCC and SCC across the UK. In the USA, the incidence is increasing by around $2 \%$ annually (Verkouteren 2017). The Australian national surveys have shown that the incidence rate has been increasing since 1985, but that it may now be reaching a plateau (Lomas 2012). There has been a very large increase in BCC incidence in young females in Europe and the USA that has led to a reversal of the male:female ratio, such that the ratio observed now is female $>$ male in the younger population (Verkouteren 2017). Higher frequency of tanning bed use and higher likelihood of seeking medical attention in females are reasons postulated to contribute to this observed trend.

Risk factors for BCC include: advanced age, male sex, fair skin, low ability to tan, intense intermittent UVR exposure during childhood, signs of actinic damage (skin changes due to excessive sun 
exposure), personal/family history of skin cancer, excessive sun bed use, phototherapy, radiotherapy, systemic immunosuppression and a genetic predisposition (Verkouteren 2017).

Germ-line mutations can also have a large effect on the risk of developing BCC such as those seen in Gorlin syndrome (naevoid BCC syndrome). This is an autosomal (non-sex determining chromosome) dominantly inherited condition characterised by developmental abnormalities and the occurrence of multiple BCCs. Mutations in people with Gorlin syndrome have been found on the PTCH1 gene located on chromosome nine, which appears to be crucial for proper embryonic development and for tumour suppression (Johnson 1996).

\section{Description of the intervention}

The primary aim of treatment is complete removal or destruction of the BCC lesion to result in cure and minimise the risk of recurrence. This should also be balanced against the patient's requirement for a good/acceptable cosmetic result (Madan 2016). There are numerous interventions currently available for BCC and the choice of intervention is determined by tumour factors such as the histological/clinical subtype of BCC, site, size, whether primary or recurrent tumour, as well as patient factors (e.g. comorbidities, importance of cosmesis) and other factors such as available resources, costs and local health economics. The different interventions are usually split into surgical and non-surgical interventions. Generally, surgical interventions are used as firstline treatments for both high-risk and low-risk BCC subtypes and non-surgical interventions are usually reserved for low-risk BCC subtypes where histological margins are less important (Madan 2016). Radiotherapy and electrochemotherapy are the exceptions as they tend to be used for high-risk BCCs not amenable to surgical intervention.

\section{Surgical (physical) interventions}

- Surgical excision (with predetermined margins)

- Surgical excision (with frozen section margin control)

- Mohs micrographic surgery (MMS - technique that takes serial horizontal frozen sections intraoperatively to examine histologically the entire surgical margin to confirm complete tumour clearance)

- Curettage and cautery (synonymous for electrodesiccation)

- Cryosurgery (synonymous for cryotherapy, delivered by a variety of methods)

- Laser therapy (ablative lasers, pulsed dye laser)

\section{Non-surgical (medical) interventions}

- Radiotherapy

- Topical imiquimod

- Topical 5-fluorouracil

- Photodynamic therapy

- Ingenol mebutate

- Intralesional interferon, fluorouracil

- Electrochemotherapy

- Others (solasodine glycosides, sinecatechins, diclofenac, calcitriol)

\section{How the intervention might work}

\section{Surgical (physical) interventions}

Surgical excision (SE) with predetermined margins is one of the most common treatments for BCC and is often regarded as the goldstandard. SE involves excising the tumour under local anaesthetic with a variable margin of clinically normal surrounding tissue. A key advantage of SE over non-surgical techniques is that it allows the histological examination of the excised specimen and therefore an accurate assessment of the surgical margins. A peripheral margin of $4 \mathrm{~mm}$ to $5 \mathrm{~mm}$ is usually suggested for well-defined, small BCC (Wolf 1987, Kimyai-Asadi 2005) and the use of curettage prior to excision to delineate the margins of the BCC is often performed (Chiller 2000). Excision through subcutaneous fat is generally advisable for the deep surgical margin, however there is a lack of evidence informing this. Factors such as the local anatomy, size and histological subtype of the BCC will influence this decision (Telfer 2008).

Mohs micrographic surgery (MMS) was pioneered in the 1930 s by Frederic Mohs and is a specialised surgical procedure in which serial horizontal frozen sections allow the microscopic examination of the entire surgical margin during surgery (in contrast to the $1 \%$ to $2 \%$ of the margin asseessed by "bread-loafing" of vertical sections examined with standard excision), so that the extent of excision can be defined precisely (Madan 2016). The technique produces very high cure rates and is often used as the gold-standard for highrisk facial BCC. Another important benefit is that it allows for tissue sparing as smaller margins than SE can be taken.

Similar to MMS is the technique of surgical excision with frozen section margin control, which employs a similar serial frozen section technique but often with standard excision margins. Frozen section margin control also differs from MMS in that excision of the tumour and an accompanying annular strip of surrounding tissue is performed with vertical margins (Nizamoglu 2016; Avril 1997).

Curettage and cautery $(C \& C)$ and curettage alone are traditional surgical BCC treatment methods that are sometimes used for small, low-risk BCCs. There is wide variation in the protocols and techniques used, including type of curette used (e.g. Volkmann spoon curette, disposable loop curette) and the number of treatment cycles. The procedure normally destroys epidermis and upper dermis and therefore scarring tends to be mild (Madan 2016). Curetted tissue is usually histologically examined but assessment of surgical margins is usually not possible.

Cryosurgery involves the direct application of liquid nitrogen to freeze a $\mathrm{BCC}$ lesion to between $-50{ }^{\circ} \mathrm{C}$ and $-60{ }^{\circ} \mathrm{C}$ resulting in destruction of both the tumour and a margin of surrounding normal tissue (Telfer 2008). Techniques and liquid nitrogen delivery methods are variable and protocols differ including number of freeze-thaw cycles. Mallon 1996 showed that a double 30-second freeze-thaw cycle achieved higher cure rates than a single freezethaw cycle for facial BCC, and that a single freeze-thaw cycle achieves high cure rates for truncal BCC.

Ablative lasers (e.g. carbon dioxide laser) utilise their tissue-heating properties to destroy BCC with high accuracy but this technique does not allow any histological examination and consequently it is not suitable for high-risk lesions. It is therefore not widely used in the treatment of BCC. There are some data suggesting that, following ablative laser treatment, recurrent $\mathrm{BCC}$ can develop into a 
more aggressive histological subtype (Jung 2011). Pulsed dye laser is generally used to treat vascular skin lesions and is also used as a treatment for thin, low-risk BCCs based on the observation that they are often highly vascular with clinically evident telangiectasias (dilated blood vessels) (Shah 2009).

\section{Non-surgical (medical) interventions}

Radiotherapy involves directing ionising radiation to destroy tumour tissue and a margin of surrounding normal tissue. Several different techniques and methods are currently used including conventional X-ray therapy, interstitial brachytherapy and superficial contact therapy with the choice of technique dependent on factors such as size and location of the lesion and performance status of the patient (Telfer 2008). Radiotherapy can be used for high-risk (including recurrent tumours) and low-risk BCCs and is often the first-line for high-risk BCCs in patients who do not want surgical intervention. Radiotherapy is usually only available at specialist hospital centres due to the high costs of the equipment and need for a clinical oncologist to decide on the treatment regimen. Usually the treatment is given in a fractionated regimen which requires multiple hospital visits over several days to weeks. Importantly, radiotherapy should not be used in the treatment of previously radiotherapy-treated recurrent BCCs and is contraindicated in patients with Gorlin syndrome as they have a increased radiosensitivity and can develop multiple BCCs within a field of radiation (Kleinerman 2009).

Imiquimod is an immunomodulator topical treatment that works by stimulating toll-like receptors- 7 and -8 , present on immune cells, inducing interferon- $\alpha$ which promotes a Th1 innate and adaptive cell-mediated immune response. This results in the recognition and subsequent destruction of tumour cells by the immune system (Dummer 2003). Imiquimod is licensed in the treatment of lowrisk $\mathrm{BCCs}$ up to $2 \mathrm{~cm}$ diameter in immunocompetent patients and the treatment regimen is five times per week for six weeks (sBCC) or 12 weeks (nBCC). It is fairly common for patients to experience influenza-like symptoms (due to the interferon release) and local application-site reactions are common.

5-fluorouracil (5-FU) is a pyrimidine analogue that disrupts DNA and RNA synthesis by inhibiting the enzyme thymidylate synthetase, thereby preventing purine and pyrimidine (DNA/RNA building blocks) from becoming incorporated into DNA during the cell-cycle (Sloan 1990). 5-FU cream is commonly used in the treatment of pre-malignant actinic keratosis (AK) and Bowen disease and is less commonly used in the treatment of small, low-risk BCC. Application site reactions are common. 5-FU can also be administered intralesionally to treat low-risk BCC.

Photodynamic therapy (PDT) is a procedure that involves the application of a photosensitiser to BCC lesions and surrounding normal skin. It relies on the principle that the sensitiser accumulates in higher concentration within rapidly proliferating malignant cells compared to cells in adjacent normal tissues. The photosensitiser is activated by visible light of certain wavelengths which produces cytotoxic oxygen species and free radicals that selectively destroy the tumour cells (Fritsch 1998). Systemic PDT is utilised for some solid organ tumours, however in dermatology the mainstay of PDT is topical using the photosensitisers 5aminolevulinic acid (ALA; Levulan ${ }^{\circledR}$ Kerastick $^{\circledR}$, Ameluz ${ }^{\circledR}$ and Alacare $\left.{ }^{\circledR}\right)$, the methyl ester of ALA, methyl aminolevulinate (MAL; Metvix ${ }^{\circledast} /$ Metvixia $^{\circledR}$ ) and more recently hexylaminolevulinate
(HAL, Hexvix ${ }^{\circledR}$ ) has been used. These photosensitisers utilise the principles of the haem biosynthesis pathway, acting as pro-drugs that enter and accumulate in cells and are metabolised to form protoporphyrin IX which accumulates in the target tissue (Ibbotson 2016). Protoporphyrin IX is a potent and efficient photosensitiser when it is present in high concentration and can be activated by light of the appropriate wavelengths (Ibbotson 2016).

Ingenol mebutate is a topical treatment derived from the plant Euphorbia peplus and is licensed in the treatment of AK. It has a dual mechanism of action whereby it destroys epidermal cells within hours and induces production of antibodies that result in neutrophils targeted to kill any residual dysplastic epidermal cells (Rosen 2012).

Intralesional interferon utilises the principles of the naturally occurring interferon family of proteins. These proteins are secreted by cells in response to viral infections and function by binding to promote Th1 innate and adaptive cell-mediated immune responses than can be harboured to destroy tumour cells (Tucker 2006). The treatment usually needs to be delivered over multiple sessions and is expensive which limits its widespread use. Systemic side-effects are common (Madan 2016).

Electrochemotherapy (ECT) involves permeating cytotoxic agents (classically bleomycin) into the tumour using short electric pulses (a process known as electroporation), resulting in a direct cytotoxic effect (Campana 2017).

Zycure cream is a mixture of $0.005 \%$ solasodine glycosides (mainly solasonine and solamargine) found in solanaceous plants of the nightshade family, such as aubergine (Punjabi 2008). The mechanism of action of these compounds is thought to involve direct cell lysis and induction of apoptosis with selectivity towards tumour cells (van der Most 2006).

Sinecatechin ointment contains epigallocatechin-3-gallate (EGCG) which is an active constituent of green tea. It is licensed in the treatment of genital warts and has been shown to have cytotoxic effects on tumours cells through inhibition of cell growth, induction of apoptosis and possible inactivation of $\beta$-catenin signalling through the Wingless (Wnt) pathway (Singh 2013).

Sonic hedgehog pathway inhibitors target the approximate $90 \%$ of $B C C$ that have mutations in genes causing unregulated activation of the sonic hedgehog pathway. Oral hedgehog pathway inhibitors (vismodegib and sonidegib) are licensed for the treatment of metastatic BCC and locally advanced BCC not appropriate for surgery or radiotherapy (Madan 2016). Trials are under way assessing the safety and efficacy of topical hedgehog inhibitors for BCCs in Gorlin syndrome patients, but none of these studies met our inclusion criteria.

\section{Intervention outcomes}

The ideal outcome of any intervention to treat a BCC would be to achieve complete clearance with no recurrence at follow-up, and to fulfil satisfactory cosmetic results with minimal risk of discomfort or adverse effects before or after the intervention. All interventions have the potential to fall short of these expectations to varying degrees. The more complete the removal or destruction of the lesion, the lower the risk of recurrence, but both modes of tumour eradication unfortunately cause localised tissue damage. The resultant inflammation can lead to adverse effects such as pain, 
erythema, bleeding, crusting, vesicles, oedema, and paraesthesia. Pain of varying degrees is one of the more common adverse effects, and therefore has the potential to influence the patient's treatment choice and/or adherence. After the initial inflammatory phase lasting a week or so, a healing wound undergoes a threeweek proliferation stage during which collagen is deposited and a scar begins to form. This is followed by a remodelling stage lasting a year, during which collagen cross-linking occurs and the scar matures (Mulholland 2012). Other undesired post-inflammatory cosmetic outcomes include hypo- and hyper-pigmentation.

\section{Why it is important to do this review}

BCC is common, continuously rising in incidence and is associated with significant morbidity and costs to healthcare systems. Although there are a wide variety of treatment modalities used in the management of BCCs, and the vast majority of the tumours are successfully treated, little research is available which accurately compares these different treatment modalities.

It is essential for both healthcare providers and patients to have the best available evidence so that they can weigh up the risks and benefits of the available treatments; to allow and promote shared decision making.

Since the publication of the previous version of this review (Bath-Hextall 2007), the results of several long-term studies on interventions for BCC have become available and several novel, non-surgical treatments have been developed and entered into clinical trials making this update timely and necessary.

\section{O B J E C T I VES}

To assess the effects of interventions for basal cell carcinoma (BCC) in immunocompetent adults.

\section{METHODS}

\section{Criteria for considering studies for this review}

\section{Types of studies}

We included published and unpublished randomised controlled trials (RCTs) of interventions for basal cell carcinoma (BCC). We aimed to identify all relevant RCTs regardless of language or publication status (published, unpublished, in press, or in progress).

\section{Types of participants}

All immunocompetent adults who have one or more histologicallyproven, primary $\mathrm{BCC}$, who were eligible for randomisation to either active treatment, placebo, other treatment or no treatment were included. Persistent (where a number of treatments have been tried with no success) or recurrent tumours have been excluded. Studies including participants with Gorlin syndrome (basal cell naevus syndrome), organoid naevi or other genetic syndromes have been excluded. Excluding such patients from trials is commonplace as the aetiology of the BCCs are different and treatments are known to work differently (for example radiotherapy can increase risk of developing further BCCs in Gorlin syndrome) and with large numbers of lesions, confounding of results can occur.

\section{Types of interventions}

Treatments for BCC including the following.

\section{Surgical (physical)}

- Surgical excision

- Mohs micrographic surgery

- Curettage and cautery

- Cryosurgery

- Laser therapy (ablative lasers, pulsed dye laser)

\section{Non-surgical (medical)}

- Radiotherapy

- Topical imiquimod

- Topical 5-fluorouracil

- Photodynamic therapy (using any photosensitiser or light source)

- Ingenol mebutate

- Intralesional interferon

- Electrochemotherapy

- Others (solasodine glycosides, tazarotene, valproic acid, sinecatechins, diclofenac, calcitriol)

\section{Types of outcome measures}

\section{Primary outcomes}

1. Recurrence at three years and five years, measured clinically (to reflect what actually happens in clinical practice). Rather than exclude potentially clinically useful data, we included recurrence data that fell outside our primary outcome time points if data were not available for three and five years.

2. Cosmetic outcome (participant- and observer-rated). We have included any validated method for assessing cosmetic outcome including the four-point scale, the Vancouver scar scale and the Patient and Observer Scar Assessment Scale (POSAS) (Mosterd 2013). We did not pre-specify a time point for our cosmetic outcome but aimed to include outcomes measured after at least one year (minimum time taken for a scar to mature). If multiple time points were reported, we reported the closest time point to one year (but not less than one year).

\section{Secondary outcomes}

1. Pain during treatment and thereafter. We included any pain reported, but prioritised outcomes that included a measure of severity e.g. 'moderate/severe pain' reported by participants as well as pain measured by visual analogue scale (VAS). We did not specify how long after treatment to measure the pain outcome but included any pain measurement if it was recorded in the follow-up period. Due to the varied nature of pain and manner in which it is recorded, we also collated pain data into Table 1.

2. Early treatment failure within six months, measured histologically.

3. Adverse effects (AEs). We did not perform numerical analyses on AEs but provided a narrative synthesis in the results and expanded on this in Table 2.

Due to the large number of studies included in this review update and to improve the overall readability of the review, we have excluded studies that did not measure any of our outcomes of interest. 


\section{Search methods for identification of studies}

We aimed to identify all relevant RCTs regardless of language or publication status (published, unpublished, in press, or in progress).

\section{Electronic searches}

For this update, we revised our search strategies in line with current Cochrane Skin practices, and expanded our list of disease terms. Details of the previous search strategies are available in BathHextall 2007.

The Cochrane Skin Information Specialist searched the following databases up to 19 November 2019:

- the Cochrane Skin Group Specialised Register using the search strategy in Appendix 1;

- the Cochrane Central Register of Controlled Trials (CENTRAL) in the Cochrane Library (2019, Issue 11) using the strategy in Appendix 2;

- MEDLINE via Ovid (from 1946) using the strategy in Appendix 3;

- Embase via Ovid (from 1974) using the strategy in Appendix 4;

- CINAHL via EBSCO (from 1981) using the strategy in Appendix 5; and

- LILACS (Latin American and Caribbean Health Science Information database from 1982) using the strategy in Appendix 6.

\section{Searching other resources}

\section{Trials registers}

Two review authors (SH and JT) searched the following trials registers up to 3 March 2019 using the term "basal cell carcinoma":

- the ISRCTN registry (www.controlled-trials.com);

- ClinicalTrials.gov (www.clinicaltrials.gov);

- the Australian New Zealand Clinical Trials Registry (www.anzctr.org.au);

- the World Health Organization International Clinical Trials Registry Platform (ICTRP) (apps.who.int/trialsearch/); and

- the EU Clinical Trials Register (www.clinicaltrialsregister.eu/).

\section{Published and unpublished trials}

We checked the reference lists of included studies for further references to eligible trials.

We contacted corresponding authors of study publications to request information on ongoing, unpublished or published trials where we needed additional information.

\section{Adverse effects}

We did not perform a separate search for adverse effects of interventions for BCC. However, we did examine data on adverse effects from the included studies we identified, and we extracted these data into a table (see Table 2).

\section{Data collection and analysis}

\section{Selection of studies}

Three review authors (FB, JT, SH) checked the titles and abstracts identified from the searches and independently assessed the full text of all RCTs of possible relevance. The review authors decided which trials fitted the inclusion criteria and recorded their methodological quality. Any disagreements were resolved by discussion between the review authors. Where there were missing data from the trial reports attempts were made to obtain that data by contacting the trial author.

\section{Data extraction and management}

Three review authors (FB, JT, SH) independently performed data extraction, using a pre-derived data extraction form for consistency. Discrepancies were resolved by a fourth review author (HW). Missing data were obtained from the trial authors where possible. Three review authors (FB, JT, SH) checked and entered the data. Data recorded included: demographics, sites, clinical types, histological diagnosis, inclusion criteria, treatment modalities and regimens, rates of recurrence, cosmetic outcomes, pain outcomes, early treatment failure rates, adverse effects data, setting and funding sources.

\section{Assessment of risk of bias in included studies}

The Cochrane 'Risk of bias' assessment framework (Higgins 2011) was used to evaluate the internal validity of studies, covering the main sources of bias across the following components:

a) the method of generation of the randomisation sequence (selection bias)

b) the method of allocation concealment - we considered it 'adequate' if the assignment could not have been foreseen (selection bias)

c) who was blinded or not blinded (participants, clinicians, outcome assessors) (performance bias and detection bias)

d) how many participants were lost to follow-up in each arm (attrition bias)

e) if there was selective outcome reporting (reporting bias)

Two authors (JT, SH) independently assessed risk of bias in included studies. Any disagreements were resolved through discussion between the authors including a third author (HW). The overall 'Risk of bias' assessment for each study's bias sources (presented in the Characteristics of included studies table) was performed in relation to our recurrence rate primary outcomes.

\section{Measures of treatment effect}

We expressed the results as risk ratio (RR) with 95\% confidence intervals (Cls) for dichotomous outcomes, and difference in means (MD) with $95 \%$ Cls for continuous outcomes.

\section{Unit of analysis issues}

The unit of analysis in the included studies was based on either participants or lesions. Where possible, data at participant level were used in preference. Where there were multiple intervention groups within a trial, we made pair-wise comparisons of similar active interventions versus placebo, or another active intervention. We analysed internally-controlled trials using appropriate techniques for paired designs (for example, for continuous outcomes using Wilcoxon Signed Ranks test or paired t-test; or for dichotomous data using McNemars test), where available, and we did not pool them with studies of other designs. 
Where dichotomous data permitted, we estimated paired odds ratios (OR) with $95 \% \mathrm{Cls}$. Where paired data could not be extracted from the papers, we presented the data narratively.

\section{Dealing with missing data}

If participant dropout led to missing data we conducted an intention-to-treat analysis, as reported in the publications of the trials. We contacted trial authors to provide missing statistics, such as standard deviations (SDs), where appropriate.

\section{Assessment of heterogeneity}

We assessed statistical heterogeneity using the $\mathrm{I}^{2}$ statistic.

\section{Assessment of reporting biases}

Study registrations and protocols were identified where available and any differences between registered and reported outcomes were flagged as potential reporting bias. We did not test publication bias as none of the comparisons in our review had the minimum number of 10 studies required in a meta-analysis to apply tests for funnel plot asymmetry (Page 2019).

\section{Data synthesis}

We calculated a weighted treatment effect (using random effects) across trials since the treatment effects for interventions are likely to vary by participant and methodological variations between studies, as per the standard methods used within Cochrane Skin. The results were expressed as RR and $95 \% \mathrm{Cl}$ for dichotomous outcomes. All analyses were conducted using RevMan 5.4.

Wherever possible, a meta-analysis was conducted to assess the overall intervention effect of different primary research studies that considered the effectiveness of the same intervention. This approach has the benefits of improving the accuracy of the results and addressing disputes between studies (Higgins 2011).

Where we performed a meta-analysis, we calculated a weighted treatment effect across trials, using a random-effects model. Where it was not possible to perform a meta-analysis, we summarised the data for each trial and have only presented forest plots. If raw data could not be extracted, we extracted the results from appropriate statistical analyses presented in the paper and reported these in the review. We considered a $\mathrm{P}$ value $<0.05$ as statistically significant.

\section{Subgroup analysis and investigation of heterogeneity}

Due to insufficient studies being included across the comparisons, we were unable able to perform any subgroup analyses.

\section{Sensitivity analysis}

We planned to conduct sensitivity analyses to examine the effects of excluding studies with a moderate or high risk of bias (Higgins 2011), but insufficient numbers of studies were included in metaanalyses.

\section{Summary of findings and assessment of the certainty of the evidence}

'Summary of findings' tables were produced for the outcomes that we determined to be the most clinically relevant. This was based on the experiences of study authors and an electronic survey sent to clinicians in our centre, on what were felt to be the most important outcomes and comparisons to patients and clinicians.
For 'Summary of findings' tables we included the following comparisons.

- MMS (Mohs micrographic surgery) versus SE (surgical excision)

- Imiquimod cream versus SE

- Radiotherapy versus SE (with or without frozen section margin control)

- MAL-PDT versus SE

- Imiquimod cream versus MAL-PDT

MMS versus SE was our main comparison based on our authors' own clinical experiences and the fact that this issue has been debated for years between dermatologists and plastics surgeons. Additionally, the experience of our centre also suggested this was the most important comparison which we established through our electronic survey.

All of our pre-defined outcomes were reported in each 'Summary of findings' table except adverse effects data which were not included. Instead these were presented as a narrative synthesis within the results and discussion sections and collated into Table 2.

'Summary of findings' tables are based on the Grading of Recommendations Assessment, Development and Evaluation (GRADE) principles (Schünemann 2013), and we used GRADEpro to assess the certainty of evidence for each review outcome. Evidence from the included RCTs was downgraded from 'high certainty' by one level for each serious study limitation found in the domains: risk of bias, inconsistency, indirectness, imprecision, and publication bias. Where confidence intervals included important benefit and serious harm (i.e. $>1.25$ and $<0.75$ ), we rated down two levels for very serious imprecision as described in the GRADE handbook (Schünemann 2013). We also rated down two levels for imprecision if there was a very wide confidence interval (more than 100 -fold difference) even if it excluded no effect (RR of 1.0). We rated down three levels when confidence intervals were so wide that we cannot have any certainty in the results and defined this as a confidence interval that includes no effect with a more than 100fold difference.

\section{Other}

A consumer (an experienced patient representative) was consulted throughout, particularly for readability and understanding of the plain language summary.

\section{RES U L T S}

\section{Description of studies}

For a full description of the studies please see the Characteristics of included studies tables.

\section{Results of the search}

The database searches (see Electronic searches) for this update retrieved a total of 4566 records. We did not identify any records of trials from our scanning of reference lists or correspondence with pharmaceutical companies (see Searching other resources). After removing 179 duplicates, we had 4387 records. We screened these and excluded 4309 records based on titles and abstracts.

We obtained the full text of the remaining 78 records. Twenty-eight studies did not meet our inclusion criteria and were excluded (see 
Characteristics of excluded studies). One study included from the previous review (Lui 2004) was also excluded as we identified that not all lesions included were histologically proven and it included a large number of Gorlin syndrome patients.

Six studies were not classified as we did not have enough information to decide if they met our eligibility criteria despite attempts to contact the authors to obtain the full study details for review (Bunker 2000; IRCT 2017 030732933N1; ISRCTN 92678315; Kang 2018; Ma 2018; RPCEC00000147; see Studies awaiting classification). Three studies are ongoing with no results available yet (EudraCT 2016-002255-25; NCT02242929; NCT03573401).

This left us with 41 records. Nine of these were related to studies included in the previous version of this review. The remaining 32 records reported 26 new studies which we included. We combined them with 26 included studies (reported in 43 references) from the previous version of the review. We then had a total of 52 included studies reported in 75 references (see Characteristics of included studies). We recorded the selection process in sufficient detail to complete a PRISMA flow diagram (Moher 2009) (Figure 1). 
Figure 1. PRISMA study flow diagram.

\section{6 studies included from previous review (reported in 43 references)}

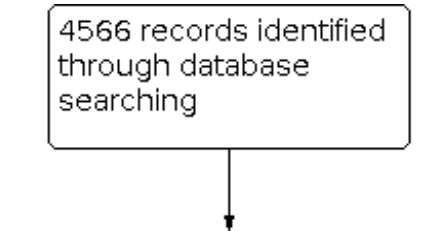

4387 records after

duplicates removed

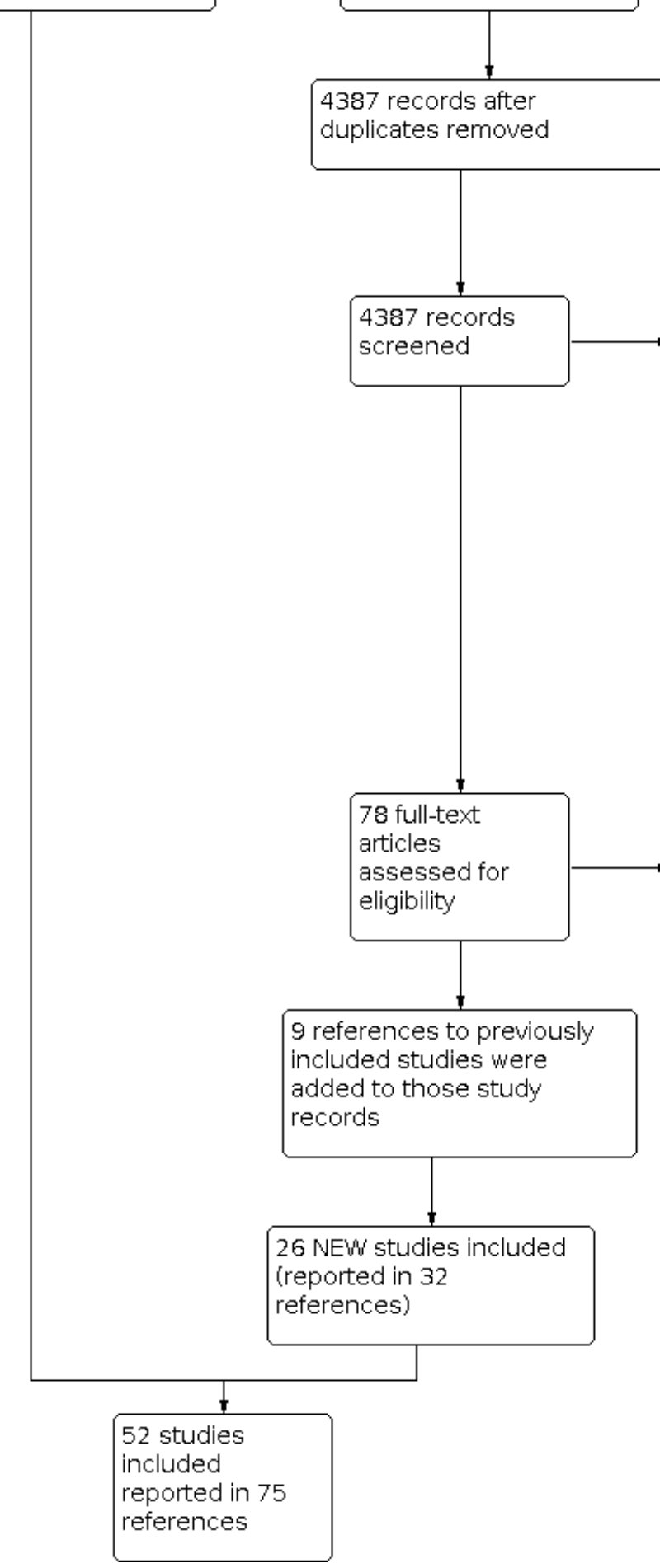

28 studies excluded from this updated review.

One previously included study (Lui 2004) was also excluded.

- 7 - no outcomes of interest measured

- 7 - not all BCCs histologically proven

- 8 - not an RCT

- 3 - not all lesions primary, previously untreated BCC

- 3 - included Gorlin syndrome

- 1 - adjuvant treatment study 6 studies awaiting classification.

3 studies ongoing.

We contacted 21 corresponding authors to obtain further information. The authors of Clover 2016 and Kuijpers 2007 kindly responded and provided further information.

\section{Included studies}

Nine papers were identified from our search that had followup data from earlier studies: Basset-Seguin 2008; Punjabi 2008; 
Rhodes 2004; Smeets 2004 (four references), Kuijpers 2007 (was Thissen 2000); Foley 2009a; Foley 2009b (was Tope 2005).

\section{Design}

All studies were prospective, RCTs with a parallel-group design. Eight RCTs were reported as pilot studies (Abd El-Naby 2019; Beutner 1999; Choi 2016; Eimpunth 2014; Haak 2015; Kuijpers 2006; Romagosa 2000; Tran 2012). The most common comparators were non-surgical treatments, with 20 RCTs comparing a non-surgical treatment against another non-surgical treatment. Fourteen RCTs compared a non-surgical treatment against placebo. Eighteen RCTs had a surgical treatment comparator, with 10 RCTs comparing a surgical treatment against a non-surgical treatment, five RCTs comparing a surgical treatment against a surgical treatment, and three RCTs comparing a surgical treatment against placebo.

Twenty-six of our included studies compared an intervention against at least one active treatment (head-to-head studies), 13 compared different regimens of the same active treatment, and 13 compared against placebo/vehicle/sham/no treatment. Seventeen studies had multiple intervention arms (three or four arms).

All intervention groups were deemed to be relevant in these multiarm studies, but due to the large number of comparisons in the review, we only presented the active treatment-arm comparisons.

The duration of the included studies ranged from six weeks to 10 years, with an average of 13 months. Just under half of the studies were multi-centre (24/52), with 26/52 single-centre studies. We were unable to ascertain the number of study centres for two studies.

\section{Setting}

All of the studies included in this review recruited participants from secondary care clinics, with the vast majority being dermatology outpatient clinics. The studies were conducted in many parts of the world. Thirty-two studies were conducted in Europe, 11 in North America, five in Australasia, three in Asia/middle East and one in South America.

\section{Participants}

A total of 6690 participants were randomised across the 52 RCTs included in this review. Studies recruited participants ranging in age from 20 to 95 years old with a median age of 64.9 years. Twelve studies did not provide data on the age of participants. Ten studies did not provide data on the gender of participants. Based on the studies that did provide data on gender, there were more male participants than females (ratio 1.48 males:1 female).

The vast majority (48/52) of studies exclusively included basal cell carcinomas (BCC) of low-risk histological subtypes (nodular, superficial). Only four studies included high-risk histological subtypes (Alpsoy 1996; Avril 1997; Clover 2016; Smeets 2004), but none of these exclusively included high-risk histological subtypes and the majority of BCCs in these studies were low-risk histological subtypes.

Most studies (27/52) included BCCs located on the head, neck and body regions. Six studies excluded BCCs located on the head and neck region (Karsai 2015; Kessels 2017; Romagosa 2000; Salmivuori 2019; Spelman 2014; Tran 2012). Nine studies included BCCs only located on the head and neck region (Abbade 2015; Abd El-Naby
2019; Avril 1997; Garcia-Martin 2011; Haak 2015; Kuijpers 2007; Landthaler 1989; Salmanpoor 2012; Smeets 2004). Three studies included BCCs exclusively on high-risk facial sites (H-zone of the face) (Smeets 2004; Haak 2015; Garcia-Martin 2011). Ten studies did not report the location of BCCs (Choi 2016; Clover 2016; Edwards 1990; Eimpunth 2014; Marks 2001; Punjabi 2008; Rogozinski 1997; Siller 2010; Soler 2000; 2005-001474-27).

\section{Sample sizes}

The number of participants randomised in each study ranged from 13 (Romagosa 2000) to 724 (Geisse 2004) with a median of 89 participants. We did not re-count the participants in the followup studies, and therefore there were a total of 6690 participants randomised in the 52 primary RCTs included in this review. Thirtyeight RCTs used participants as the unit of analysis, and 14 RCTs used lesions as the unit of analysis. A total of 7241 lesions were included in this review.

\section{Funding}

Overall, 22 studies were industry funded, with studies of imiquimod and photodynamic therapy being over-represented in this group. Seventy-nine per cent of studies (11/14) assessing imiquimod were funded by the same company (3M), and seven out of 16 PDT studies were industry-funded (five by PhotoCure/Galderma).

\section{Interventions}

The trials fell into 15 broad therapeutic categories. For a full description of the studies, please see the Characteristics of included studies table. ${ }^{*}$ Denotes study new to this update.

\section{Surgical excision (SE)}

Ten RCTs.

- Mohs micrographic surgery versus SE (Smeets 2004)

- Imiquimod versus SE (Bath-Hextall 2014*)

- Radiotherapy versus SE (with option for frozen section margin control) (Avril 1997)

- Curettage versus curettage and cautery versus SE (Salmanpoor 2012*)

- Curettage and cryosurgery versus SE (Kuijpers 2007*)

- MAL-PDT versus SE (Abbade 2015*; Rhodes 2004; Szeimies 2008)

- ALA-PDT versus SE (Mosterd 2008*)

- Electrochemotherapy versus SE (Clover 2016*)

\section{Radiotherapy}

Four RCTs.

- Radiotherapy versus SE (Avril 1997)

- Imiquimod versus radiotherapy (Garcia-Martin 2011*)

- Low dose versus high-dose radiotherapy (Landthaler 1989*)

- Radiotherapy versus cryosurgery (Hall 1986)

\section{Cryosurgery}

Four RCTs.

- Radiotherapy versus cryosurgery (Hall 1986)

- Curettage and cryosurgery versus SE (Kuijpers 2007)

- PDT versus cryosurgery (Wang 2001; Basset-Seguin 2008*) 


\section{Curettage and cautery (C\&C)}

One RCT.

- Curettage versus curettage and cautery versus SE (Salmanpoor $2012^{\star}$ )

\section{Photodynamic therapy (PDT)}

Sixteen RCTs.

- MAL-PDT versus SE (Abbade 2015*; Rhodes 2004; Szeimies 2008)

- ALA-PDT versus SE (Mosterd 2008*)

- ALA-PDT versus MAL-PDT (Kuijpers 2006*)

- MAL-PDT versus HAL-PDT versus BF200-ALA-PDT (Salmivuori $2019^{\star}$ )

- BF-200 ALA-PDT versus MAL-PDT (Morton 2018*)

- Two-fold ALA-PDT versus conventional MAL-PDT (Kessels 2018*)

- PDT versus cryosurgery (Wang 2001; Basset-Seguin 2008)

- Imiquimod cream versus MAL-PDT (Arits 2013*)

- 5-FU cream versus MAL-PDT(Arits 2013*)

- Laser ALA-PDT versus broadband halogen light ALA-PDT (Soler 2000)

- Ablative fractional laser-assisted MAL-PDT versus MAL-PDT (Choi 2016*; Haak 2015*)

- MAL-PDT versus placebo (Foley 2009a; Foley 2009b)

\section{Imiquimod ( $5 \%$ cream unless otherwise stated)}

Fourteen RCTs.

- Imiquimod versus MAL-PDT (Arits 2013*)

- Imiquimod versus 5-FU cream (Arits 2013*)

- Imiquimod versus SE (Bath-Hextall 2014*)

- Imiquimod versus radiotherapy (Garcia-Martin 2011*)

- Dose response trial of imiquimod with and without occlusion for BCC - one trial for sBCC and one trial for nBCC (Sterry 2002a; Sterry 2002b)

- Five different doses of imiquimod for $\mathrm{sBCC}$ and $\mathrm{nBCC}$ versus vehicle (Beutner 1999)

- Open-label dose response trial of imiquimod for primary superficial BCC (Marks 2001)

- Open-label dose response trial of imiquimod for nBCC (Shumack 2002a)

- Dose response trial of imiquimod versus vehicle for sBCC (Geisse 2002; Geisse 2004; Schulze 2005; Shumack 2002b)

- Two different doses of imiquimod (Ezughah 2008*; Eigentler $2007^{\star}$ )

\section{Fluorouracil (5-FU)}

Three RCTs.

- 5-FU cream versus MAL-PDT (Arits 2013*)

- Imiquimod cream versus 5-FU cream (Arits 2013*)

- 5-FU cream in phosphatidyl choline versus 5-FU cream in petrolatum (Romagosa 2000)

- Varying treatment regimens of 5-FU/epinephrine injectable gel (Miller 1997)

\section{Electrochemotherapy (ECT)}

One RCT.

- Electrochemotherapy versus SE (Clover 2016*)

\section{Ingenol mebutate}

Two RCTs.

- Ingenol mebutate gel $0.0025 \%$ versus ingenol mebutate gel $0.01 \%$ versus ingenol mebutate gel $0.05 \%$ versus vehicle gel (Siller 2010*)

- Ingenol mebutate gel + full occlusive aluminium disk versus ingenol mebutate gel and semi-occlusive dressing with Opsite or Ingenol mebutate gel with no occlusion (Spelman 2014*)

\section{Solasodine glycosides}

One RCT.

- Solasodine glycoside (Zycure) cream versus matching vehicle (Punjabi 2008)

\section{Topical valproic acid gel and tazarotene gel}

One RCT.

- Valproic acid (Baceca ${ }^{\circledR}$ ) gel followed by Tazarotene gel versus placebo gel followed by Tazarotene gel (placebo cross-over after eight weeks) (2005-001474-27*)

\section{Topical diclofenac and vitamin D}

One RCT.

- Diclofenac-3\% gel versus calcitriol 3ug/g ointment versus diclofenac-3\% gel plus calcitriol $3 \mathrm{ug} / \mathrm{g}$ ointment versus no topical treatment (Brinkhuizen 2016*)

\section{Pulsed dye laser (PDL)}

Four RCTs.

- PDL versus sham (Karsai 2015*)

- PDL single session versus PDL two sessions (Abd El-Naby 2019*)

- PDL $7 \mathrm{~mm}$ spot-size, single pulses versus PDL $10 \mathrm{~mm}$ spot-size, stacked pulses (Tran 2012*)

- PDL $10 \mathrm{~mm}$ spot-size, one stacked pulse session versus no treatment (Eimpunth 2014*)

\section{Intralesional interferon (IFN) therapy}

Four RCTs.

- IFN alpha-2a, 2b or alpha-2a and 2b (Alpsoy 1996)

- IFN alpha 2b versus vehicle (Cornell 1990)

- Number of dosages of IFN alpha 2b (Edwards 1990)

- IFN beta versus placebo (Rogozinski 1997)

\section{Topical sinecatechins}

One RCT.

- Sinecatechin $10 \%$ ointment versus vehicle (Kessels $2017^{\star}$ ) 


\section{Outcomes}

Out of the 52 comparisons, only $21 \%(11 / 52)$ reported our 3year recurrence primary outcome and only $17 \%(9 / 52)$ reported our 5-year recurrence primary outcome. Cosmetic outcome data were reported for $37 \%$ (19/52) of the comparisons (14/52 had data available for analysis). Early treatment failure was reported in $75 \%$ (39/52) of comparisons. Pain data were reported in $46 \%(24 / 52)$ of the comparisons (10/52 had data available for analysis). Adverse effects data were reported for most comparisons (81\%, 42/52), but due to their heterogeneity we did not perform any analyses and instead summarised these data in Table 2.

\section{Excluded studies}

We excluded 29 studies due to the following reasons: no outcomes of interest measured (8); not all lesions histologically proven (7); not an RCT (8); not all lesions primary, untreated BCC (3); inclusion of Gorlin syndrome participants (3) and a study on adjuvant treatment (1). Please see the Characteristics of excluded studies tables for further details.

\section{Studies awaiting classification}

We were unable to make a final decision on whether to include or exclude six studies despite attempts to contact the study investigators. See the Studies awaiting classification tables for further details.

\section{Ongoing studies}

Three studies were identified that are currently ongoing and had no data available for us to include in this review. See the Ongoing studies tables for further details.

\section{Risk of bias in included studies}

In order to objectively assess risk of bias, we used Cochrane 'Risk of bias' methods (Higgins 2011). Occasionally, our ability to assess risk was hampered by limited reporting of data. Where there was inadequate information to make a judgement, for example abstract-only studies, attempts to contact the authors were made in order to get access to the data needed. Please see the Characteristics of included studies tables for full details. Figure 2 and Figure 3 summarise the overall data.

Figure 2. 'Risk of bias' graph: review authors' judgements about each risk of bias item presented as percentages across all included studies.

Random sequence generation (selection bias)

Allocation concealment (selection bias)

Blinding of participants and personnel (performance bias): All outcomes

Blinding of outcome assessment (detection bias): All outcomes

Incomplete outcome data (attrition bias): All outcomes

Selective reporting (reporting bias)

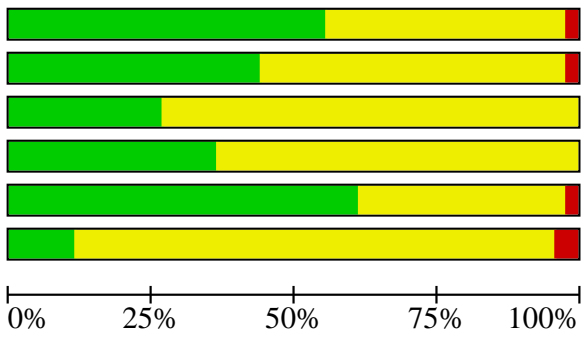

Low risk of bias

Unclear risk of bias

High risk of bias 
Figure 3. 'Risk of bias' summary: review authors' judgements about each risk of bias item for each included study.

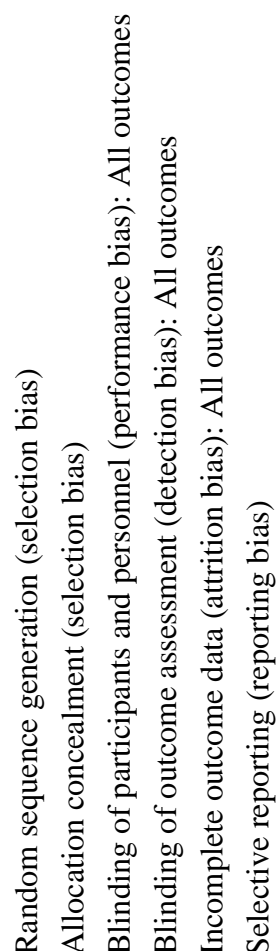

\begin{tabular}{|c|c|c|c|c|c|c|}
\hline 2005-001474-27 & $?$ & $?$ & + & + & + & \\
\hline Abbade 2015 & + & $?$ & $?$ & $?$ & $?$ & $?$ \\
\hline Abd El-Naby 2019 & + & + & $?$ & + & + & $?$ \\
\hline Alpsoy 1996 & $?$ & $?$ & $?$ & $?$ & + & $?$ \\
\hline Arits 2013 & + & + & $?$ & + & $?$ & $?$ \\
\hline Avril 1997 & $?$ & $?$ & $?$ & $?$ & + & $?$ \\
\hline Basset-Seguin 2008 & + & $?$ & $?$ & $?$ & $?$ & $\odot$ \\
\hline Bath-Hextall 2014 & + & + & $?$ & $?$ & + & + \\
\hline Beutner 1999 & $?$ & $?$ & + & + & + & $?$ \\
\hline Brinkhuizen 2016 & + & + & $?$ & $?$ & $?$ & + \\
\hline Choi 2016 & + & $?$ & $?$ & + & + & $?$ \\
\hline Clover 2016 & + & + & $?$ & $?$ & $?$ & $?$ \\
\hline Cornell 1990 & + & $?$ & $?$ & $?$ & $?$ & $?$ \\
\hline Edwards 1990 & $?$ & $?$ & $?$ & $?$ & + & $?$ \\
\hline Eigentler 2007 & $?$ & $?$ & + & $?$ & $?$ & $?$ \\
\hline Eimpunth 2014 & $?$ & $?$ & $?$ & $?$ & + & $?$ \\
\hline Ezughah 2008 & + & + & $?$ & + & + & $?$ \\
\hline Foley 2009a & + & + & + & + & + & $?$ \\
\hline Foley $2009 b$ & + & + & + & + & + & $?$ \\
\hline Garcia-Martin 2011 & & 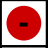 & $?$ & $?$ & + & $?$ \\
\hline Geisse 2002 & + & $?$ & + & + & + & $?$ \\
\hline Geisse 2004 & + & + & + & $?$ & $?$ & $?$ \\
\hline Haak 2015 & 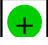 & 11 & ? & + & ? & \\
\hline
\end{tabular}


Figure 3. (Continued)

\begin{tabular}{|c|c|c|c|c|c|c|}
\hline Geisse 2 & 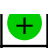 & + & + & & & \\
\hline Haak 2015 & + & + & $?$ & + & $?$ & \\
\hline Hall 1986 & $?$ & $?$ & $?$ & $?$ & $?$ & 9 \\
\hline Karsai 2015 & + & + & + & $?$ & + & \\
\hline Kessels 2017 & + & + & + & + & + & \\
\hline Kessels 2018 & + & + & $?$ & + & + & \\
\hline Kuijpers 2006 & $?$ & $?$ & + & + & + & $?$ \\
\hline Kuijpers & + & + & $?$ & $?$ & $?$ & \\
\hline adtha & $?$ & $?$ & $?$ & $?$ & + & \\
\hline $\mathrm{Ma}$ & $?$ & $?$ & $?$ & $?$ & + & \\
\hline Mill & $?$ & $?$ & $?$ & $?$ & $?$ & \\
\hline Morto & + & + & $?$ & + & + & $1+$ \\
\hline Mosterd 2008 & + & + & $?$ & $?$ & $?$ & $?$ \\
\hline Punjabi 2008 & $?$ & $?$ & + & + & - & $?$ \\
\hline Rho & + & + & $?$ & $?$ & $?$ & $?$ \\
\hline gozir & $?$ & $?$ & $?$ & $?$ & $?$ & 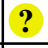 \\
\hline Romago & $?$ & $?$ & + & + & + & $?$ \\
\hline anpo & $?$ & $?$ & $?$ & $?$ & $?$ & $?$ \\
\hline almivu & $?$ & $?$ & $?$ & $?$ & $?$ & $?$ \\
\hline Schu & + & + & + & + & + & 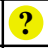 \\
\hline Shuma & $?$ & $?$ & $?$ & $?$ & $?$ & \\
\hline b & $?$ & $?$ & $?$ & $?$ & + & \\
\hline 0 & + & + & $?$ & + & + & \\
\hline Sme & + & + & $?$ & $?$ & + & $?$ \\
\hline 00 & $?$ & + & $?$ & $?$ & + & $?$ \\
\hline Spelm & $?$ & $?$ & $?$ & $?$ & $?$ & $?$ \\
\hline Ster & + & $?$ & $?$ & $?$ & + & $?$ \\
\hline $2 b$ & + & $?$ & $?$ & $?$ & + & $?$ \\
\hline Szeimies 2008 & + & + & $?$ & $?$ & + & 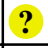 \\
\hline Tran 2012 & $?$ & $?$ & + & + & + & $?$ \\
\hline Wang 2001 & + & + & $?$ & $?$ & + & $1 \cdot$ \\
\hline
\end{tabular}

\section{Allocation}

\section{Random sequence generation and concealment of allocation}

The randomisation process in general and concealment of allocation are the most important and sensitive indicators that bias has been minimised in a clinical trial (Schulz 1995).

Twenty-nine studies had a low risk of bias for random sequence generation and 22 studies had an unclear risk of bias. Twenty-three studies had a low risk of bias for allocation of concealment whereas 28 studies had an unclear risk of bias. Only one study (Garcia-Martin 2011) had a high risk of bias for random sequence generation and allocation of concealment.

\section{Blinding}

Only 14 studies were rated as having a low risk of bias for blinding of participants/personnel with the remaining 38 studies being assessed as having an unclear risk of performance bias. Unclear risk of bias was chosen in three situations: 1) if the blinding method was not clearly stated by the study authors 2) the study was unblinded 3 ) the study was blinded but due to the nature of the interventions it is likely that participants/personnel would be aware of the treatment allocation. The reason we decided to rate all these situations as "unclear" is that it is unclear how lack of blinding would impact our outcomes of interest.

Only 19 studies were assessed as low risk of bias for blinding of the outcome assessor. Thirty-three studies were assessed as having unclear risk of detection bias. We rated the bias as unclear for the same situations as detailed above for performance bias. Even if studies reported that outcome assessors were blinded, for all the studies comparing a surgical intervention against a non-surgical intervention, we felt that it was not possible to truly blind due to the nature of the interventions and again we were unclear whether being unblinded would bias the assessing of clinical response with regards to recurrence rates. However we decided that being unblinded would bias the rating of cosmetic outcomes and in this situation we consider the risk of bias to be high. As recurrence 
rate is our primary outcome the risk of bias rating displayed in the Characteristics of included studies table relates to this.

\section{Incomplete outcome data}

Analysis should be performed according to intention-to-treat principle to minimise risk of attrition bias (Fergusson 2002). However, in many of the trials analysis of outcome was carried out only in those participants who completed the trial. Thirtytwo studies had a low risk of bias for incomplete outcome data. Nineteen studies had an unclear risk and one study (Punjabi 2008) had a high risk of attrition bias as there was a large difference in the number of participants that withdrew from the study between groups and the authors used the "last observation carried forward" method to deal with missing data which is an imputation method that fails to acknowledge uncertainty in the imputed values (Higgins 2011).

\section{Selective reporting}

To minimise the risk of selective outcome reporting bias, outcomes for studies should all be specified prior to recruitment in a time-stamped, publicly available trial registry. This has been a prerequisite for publication since 2005 for publication in International Committee of Medical Journal Editors (ICMJE) member journals. Only 11 studies prospectively registered their primary outcomes and of these only six were rated as low risk of selective reporting bias which is a concerningly low number. Fortyfour studies were rated as unclear risk of bias and two studies were reported as having a high risk of reporting bias: 2005-001474-27 and Basset-Seguin 2008.

\section{Effects of interventions}

See: Summary of findings 1 Mohs micrographic surgery compared to surgical excision for basal cell carcinoma of the skin; Summary of findings 2 Imiquimod compared to surgical excision for basal cell carcinoma of the skin; Summary of findings 3 Radiotherapy compared to surgical excision (with or without frozen section margin control) for basal cell carcinoma of the skin; Summary of findings 4 MAL-PDT compared to surgical excision for basal cell carcinoma of the skin; Summary of findings $\mathbf{5}$ Imiquimod cream compared to MAL-PDT for basal cell carcinoma of the skin

Not all of the comparisons measured or reported all primary and secondary outcomes. If an outcome has not been reported under a comparison, this means it was not measured by any study.

\section{Surgical excision}

\section{Mohs micrographic surgery (MMS) versus surgical excision (SE)}

One study (Smeets 2004) compared SE against MMS in 374 participants (408 lesions) with high-risk facial primary basal cell carcinomas (BCCs) (high-risk histological subtype or located in the ' $\mathrm{H}$-zone' of the face or both). The study also randomised 204 recurrent facial BCCs but we have not included these data as we are only interested in primary BCC. The study used $3 \mathrm{~mm}$ margins for both treatments to standardise the two modalities (smaller margins are usually used for MMS). If the defect margins were found to be positive after SE, a re-excision with a $3 \mathrm{~mm}$ margin was done. If the margins remained positive after a second excision then MMS was undertaken. The primary outcome was recurrence at 30 months (which we have considered to be approximately three years). Recurrence at five years and 10 years were published in two further papers. See Summary of findings 1.

\section{Primary outcomes}

\section{Recurrence}

There may be slightly fewer recurrences with MMS and SE at three years (1.9\%, 3/160 versus $2.9 \%, 5 / 171$, respectively) (risk ratio (RR) $0.64,95 \%$ confidence interval $(\mathrm{Cl}) 0.16$ to 2.64 ; Analysis 1.1 ), and at five years $(3.2 \%, 4 / 125$ versus $5.2 \%, 7 / 134$, respectively) (RR 0.61 , $95 \% \mathrm{Cl} 0.18$ to 2.04 ; Analysis 1.2 ). We rated the certainty of evidence as low for both these outcomes, rating down two levels for very serious imprecision due to the very wide $95 \% \mathrm{Cls}$. This $\mathrm{Cl}$ indicates that the true MMS recurrence rate may represent an important improvement or worsening of recurrence rates when compared to SE.

\section{Cosmetic outcome (participant- and observer-rated).}

The authors state that overall, participant- and observer-rated cosmetic outcomes did not significantly differ between MMS and SE when measured at 18 months. We rated the certainty of evidence as low, rating down two levels for very serious indirectness as although the authors did compare the cosmetic outcomes between the two groups, they did not present the data for analysis.

\section{Secondary outcomes}

\section{Adverse effects}

The study reported in their text that there was quote: "no difference in post-operative complications between SE and MMS"; however raw data were not presented for this outcome to verify this. See Table 2 for further adverse effect (AE) data from this study.

\section{Imiquimod cream versus surgical excision (SE)}

One study (Bath-Hextall 2014) compared imiquimod to SE in 501 participants with nodular BCC ( $\mathrm{nBCC}$ ) or superficial BCC (sBCC) at low-risk sites in a non-inferiority trial design with five-year followup. Participants randomised to imiquimod treatment applied the cream daily for six weeks (sBCC) or 12 weeks (nBCC). The licensed imiquimod regimen for SBCC is five days a week for six weeks and for $\mathrm{nBCC}$ is five days a week for 12 weeks. SE margins were $4 \mathrm{~mm}$. See Summary of findings 2.

\section{Primary outcomes}

\section{Recurrence}

Imiquimod probably results in more recurrences $(16.4 \%, 35 / 213)$ than SE $(1.6 \%, 3 / 188)$ at three years corresponding to a 10 -fold increased risk of recurrence with imiquimod (RR $10.30,95 \% \mathrm{Cl}$ 3.22 to 32.94 ; Analysis 2.1). We rated the certainty of evidence as moderate, rating down for serious imprecision due to the wide $95 \%$ $\mathrm{Cl}$ and because this was only a single study.

By five years, imiquimod may result in more recurrences $(17.5 \%$, $36 / 206)$ compared with SE $(2.3 \%, 4 / 177)$ with a nearly 8 fold increased risk of recurrence ( $\mathrm{RR} 7.73,95 \% \mathrm{Cl} 2.81$ to 21.30 ; Analysis 2.2). We rated the certainty of evidence as moderate, rating down for imprecision (wide $95 \% \mathrm{Cl}$, single study).

\section{Cosmetic outcome (participant- and observer-rated)}

Cosmetic outcome was assessed at six months and three years by participants and two independent dermatologists using a six-point 
scale (unable to see lesion, very poor, poor, fair, good, excellent). Our analyses focused on using data from three-year follow-up due to the reasons outlined in the methods section.

When participant-rated, there may be little to no difference between imiquimod $(91.9 \%, 147 / 160)$ and SE $(92.2 \%, 153 / 166)$ on the rate of good/excellent cosmetic outcomes (RR 1.00, 95\% Cl 0.94 to 1.06; Analysis 2.3). When dermatologist-rated, imiquimod may improve rate of good/excellent cosmetic outcomes $(60.6 \%$, $103 / 170)$ compared to SE $(35.6 \%, 62 / 174)$, corresponding to a $70 \%$ increase rate for imiquimod (RR $1.70,95 \% \mathrm{Cl} 1.35$ to 2.15 ; Analysis 2.3). We rated the certainty of evidence as low for both these comparisons, rating down for serious risk of bias (unable to truly blind due to nature of interventions) and serious risk of imprecision (only one study).

\section{Secondary outcomes}

\section{Pain during and after treatment}

Patient diaries were used to record information about the level of pain felt before and after the lesion was treated. During treatment, imiquimod probably worsens the risk of moderate/severe pain compared to SE (30\%, $72 / 242$ versus $22 \%, 44 / 201$, respectively) corresponding to a $36 \%$ increased risk with imiquimod (RR 1.36, $95 \% \mathrm{Cl} 0.98$ to 1.88 ; Analysis 2.4). We rated the certainty of evidence as low, rating down for serious imprecision (wide $95 \% \mathrm{Cl}$, including the null effect which indicates there may be no difference between the two treatments) and serious risk of attrition bias.

However, during the 16-week follow-up period imiquimod probably reduces the risk of moderate/severe pain compared to SE $(9 \%$, $22 / 233$ versus $20 \%, 41 / 206$ ) corresponding to a $53 \%$ decreased risk with imiquimod (RR $0.47,95 \% \mathrm{Cl} 0.29$ to 0.77 ; Analysis 2.4). We rated the certainty of evidence as low, rating down for serious risk of imprecision (only one study) and serious risk of attrition bias. See Table 1 for further pain data from this study.

\section{Adverse effects}

There were slightly more mild/moderate AEs reported during the first six months of treatment in the imiquimod group compared to the SE group ( $94 \%$ of participants versus $88 \%$, respectively). Itching at the tumour site was frequently reported (85\%, 211/249 imiquimod versus 56\%, 129/229 SE), but weeping at the tumour site was also common $(64 \%, 160 / 249$ for imiquimod versus $35 \%$, $81 / 229$ for SE). Five participants treated with imiquimod withdrew from the study because of AEs related to the treatment whereas no participants withdrew because of AEs related to SE. Thirty-eight $(15 \%)$ of participants needed a dose reduction in the imiquimod group due to AEs. See Table 2 for further AE data.

\section{Radiotherapy versus surgical excision (SE) with or without frozen section margin control}

One study (Avril 1997) involving 347 participants (347 lesions) compared SE (at least $2 \mathrm{~mm}$ margin with the option of frozen section margin control) against radiotherapy in primary BCCs of the face, involving tumours less than $4 \mathrm{~cm}$ in diameter. BCCs included nodular, ulcerated, superficial and pagetoid and morphoeaform. The main outcome measure was histologicallyconfirmed persistent tumour or recurrent disease at four years. Three radiation techniques were used: interstitial brachytherapy ( $n=95)$, superficial contact therapy $(n=50)$ and conventional radiotherapy $(n=20)$. See Summary of findings 3 .

\section{Primary outcomes}

\section{Recurrence}

At three years the recurrence rates were 5.2\% (9/173) and 0\% $(0 / 174)$ for radiotherapy and SE, respectively. (RR 19.11, 95\% Cl 1.12 to 325.78; Analysis 3.1). We rated the certainty of evidence as low, rating down two levels for very serious imprecision due to the very wide $95 \% \mathrm{Cl}$, which although does not include the null effect, indicates that radiotherapy may lead to a slight or markedly increased risk of recurrence when compared to SE.

As the study set their primary outcome of recurrence at four years, we have included these data. By four years, radiotherapy may result in a higher risk of recurrence compared to SE with a recurrence rate of $6.4 \%(11 / 173)$ versus $0.6 \%(1 / 174)$, respectively (RR $11.06,95 \%$ $\mathrm{Cl} 1.44$ to 84.77 ; Analysis 3.2). We rated the certainty of evidence as low, rating down for serious indirectness as the outcome was measured outside our three-year and five-year time points and for serious imprecision due to the wide $95 \% \mathrm{Cl}$.

\section{Cosmetic outcome (participant- and observer-rated)}

Comparing the rate of participant-reported good cosmetic outcomes (three-point scale: bad, fair or good) at four years between the groups, radiotherapy probably worsens the rate of good cosmetic outcome compared to SE frozen (RR 0.76, 95\% Cl 0.63 to 0.91 ; Analysis 3.3). At four years, radiotherapy probably worsens the rate of dermatologist-assessed cosmesis based on the scar (bad, clearly marked or slightly visible) compared to SE (RR $0.48,95 \% \mathrm{Cl} 0.37$ to 0.62 ; Analysis 3.3 ). We rated the certainty of evidence for both these outcomes as moderate, rating down for serious risk of bias (high risk of blinding bias). We performed an intention-to-treat analyses on both these outcomes (as detailed in the methods section) due to fairly large dropout numbers at four years.

\section{Secondary outcomes}

\section{Adverse effects}

After radiotherapy, dyspigmentation and telangiectasia (which may affect the cosmetic appearance) developed in more than $65 \%$ of the participants by four years. Radiodystrophy affected $41 \%$ of the participants at four years and $5 \%$ of the patients in the radiotherapy group had necrosis. These AEs were exclusive to the radiotherapy group. The main characteristics of surgical scars were deformations and constrictions and although these decreased with time they still affected $25 \%$ and $5 \%$ of participants, respectively at four years. See Table 2 for further AE data for this study.

\section{Radiotherapy}

\section{Low-dose versus high-dose radiotherapy}

Landthaler 1989 compared low-dose (48 Gy) against high-dose radiotherapy (60 Gy) in 319 participants with 290 BCCs (unclear subtype) and 44 SCCs located on the face.

\section{Secondary outcomes}

\section{Early treatment failure}

There may be little to no difference in the risk of early treatment failure between low dose radiotherapy $(8.1 \%, 12 / 148)$ and high dose radiotherapy $(9.6 \%, 14 / 142$ ) (RR $0.82,95 \% \mathrm{Cl} 0.39$ to 1.72 ; Analysis 7.1). The certainty of evidence was low due to very serious imprecision where the $95 \% \mathrm{Cl}$ indicates that low dose might lead to 
an important reduction or an important increase in early treatment failures.

\section{Cryosurgery}

\section{Radiotherapy versus cryosurgery}

One study (Hall 1986) of 93 participants (93 lesions) compared radiotherapy against cryosurgery (unclear subtype, but lesions on nose/ears were excluded). Radiotherapy was applied with $130 \mathrm{kV}$ $\mathrm{X}$-rays and fractionation depended on lesion size. Cryosurgery was performed with two freeze-thaw cycles each lasting one minute.

\section{Primary outcomes}

\section{Recurrence}

Radiotherapy may reduce the risk of recurrence $(4.1 \%, 2 / 49)$ compared to cryosurgery $(38.6 \%, 17 / 44)$ at 2 years follow-up (RR $0.11,95 \% \mathrm{Cl} 0.03$ to 0.43 ; Analysis 8.1 ). However, we rated the certainty of evidence as low, rating down for serious indirectness (as the outcome was measured at two years) and serious imprecision (single study with a fairly wide $95 \% \mathrm{Cl}$ ).

\section{Cosmetic outcome (participant- and observer-rated)}

The study reported that there was no statistically significant difference in cosmetic outcomes between the interventions; however, the raw data were not presented to verify this.

\section{Secondary outcomes}

\section{Pain during and after treatment}

The study reported that the degree of pain reported by patients (on questionnaires given during follow-up) was not statistically significant between the two interventions; however, the data were not presented for further analyses.

\section{Adverse effects}

Hypopigmentation was more common than hyperpigmentation with both modes of treatment ( $81 \%$ of those in the radiotherapy group and $88 \%$ of those in the cryosurgery group). Seven participants treated with radiotherapy developed some radiation telangiectasia. Hypopigmentation and telangiectasia tend to be lifelong. Five participants treated with cryosurgery developed milia - these all resolved by one year. See Table 2 for further AE data from this study.

\section{Curettage and cryosurgery versus surgical excision}

One study (Kuijpers 2007) compared curettage and cryosurgery against SE in 88 participants with 100 low-risk BCCs on the head and neck region ( $96 \mathrm{nBCC}$, four SBCC) with five years follow-up. Cryosurgery was performed with two 20 -second freeze-thaw cycles using a neoprene cone to give a free margin of $5 \mathrm{~mm}$ around the tumour. Surgery was performed with $3 \mathrm{~mm}$ margins. See Table 3.

\section{Primary outcomes}

\section{Recurrence}

At three years, curettage and cryosurgery may slightly increase the risk of recurrence compared to SE $(13.7 \%, 7 / 51$ versus $4.1 \%$, 2/49, respectively) (RR 3.36, 95\% Cl 0.73 to 15.40; Analysis 9.1). We rated the certainty of evidence as low, downgrading two levels for very serious imprecision (very wide $95 \% \mathrm{Cl}$ that indicates both important benefit and harm).
At five years cryosurgery probably increases the risk of recurrence compared to SE (23.7\% versus $8.5 \%$ ) (RR $2.78,95 \% \mathrm{Cl} 0.93$ to 8.34; Analysis 9.2). We rated the certainty of evidence as moderate, downgrading one level for serious imprecision (wide $95 \% \mathrm{Cl}$ that includes important benefit and little to no difference).

\section{Cosmetic outcome (participant- and observer-rated)}

Cosmetic outcomes were assessed by observers and patients at one year post treatment on a three-point scale (bad, fair, good). When participant-rated, curettage and cryosurgery may slightly decrease the rate of good cosmetic outcomes compared with SE (RR 0.88, $95 \% \mathrm{Cl} 0.78$ to 0.98 ; Analysis 9.3).

When dermatologist-rated, curettage and cryosurgery may decrease the rate of good cosmetic outcome compared to SE (RR $0.28,95 \% \mathrm{Cl} 0.16$ to 0.47 ; Analysis 9.3). We rated the certainty of evidence as low for both of these outcomes rating down for serious risk of blinding bias (unable to truly blind due to nature of interventions) and serious risk of imprecision (single study).

\section{Secondary outcomes}

\section{Adverse effects}

Secondary wound infection, treated with systemic antibiotics, was seen in 5.9\% (3/51) participants in the curettage and cryosurgery group. In the SE, group systemic antibiotics were needed for secondary wound infection in $8.2 \%$ (4/49) of participants in the first and second weeks after treatment. No further AE data from this study were available.

\section{Currettage and cautery}

\section{Curettage and cautery versus curettage versus surgical excision}

One small study of 55 participants with 69 BCCs on the face and scalp (majority $\mathrm{nBCC}$ ) compared: curettage and cautery (C\&C) versus $\mathrm{SE}$, curettage versus SE and curettage versus curettage and cautery with just two years follow-up (Salmanpoor 2012).

\section{Primary outcomes}

\section{Recurrence}

It is uncertain whether the risk of recurrence is improved for curettage versus SE (RR 2.40, 95\% Cl 0.49 to 11.77; Analysis 4.1), C\&C versus SE (RR 0.96, 95\% Cl 0.15 to 6.28; Analysis 5.1), and curettage versus C\&C (RR $0.40,95 \% \mathrm{Cl} 0.08$ to 1.97 ; Analysis 6.1 ). We rated the certainty of evidence as very low for all these comparisons, rating down for very serious imprecision (very wide $95 \% \mathrm{Cls}$ ) and also for serious indirectness as the study only measured recurrence up to two years.

\section{Photodynamic therapy (PDT)}

\section{PDT versus surgical excision (SE)}

Four studies compared PDT with SE, three of which used the photosensitiser methyl aminolevulinate (MAL-PDT) (Rhodes 2004; Szeimies 2008; Abbade 2015), and one which used the photosensitiser 5-aminolevulinate (ALA-PDT) (Mosterd 2008).

Rhodes 2004 compared MAL-PDT against SE for nBCC of the face in 103 participants (118 lesions) with five years follow-up. Abbade 2015 compared MAL-PDT against SE in 57 participants (68 lesions) 
with $\mathrm{nBCC}$ in the head and neck area with follow-up over three years.

Szeimies 2008 was a non-inferiority study that compared MAL-PDT with SE in 196 participants with $246 \mathrm{sBCCs}$ (between $8 \mathrm{~mm}$ and $20 \mathrm{~mm}$ located anywhere except mid-face) with one year followup. The inferiority margin was set at a difference of $15 \%$ in terms of per cent reduction in lesion count three months after the last treatment.

Mosterd 2008, compared fractionated ALA-PDT with SE in 171 primary nBCCs (149 participants) with five years follow-up.

All the MAL-PDT studies followed the standard European PDT protocol which defines one cycle as two PDT sessions separated by a one-week interval. The ALA-PDT (Mosterd 2008) study did two illuminations on the same day separated by 60 minutes and any incomplete responses were re-treated surgically. Rhodes 2004 and Szeimies 2008 performed a gentle curettage of lesions to remove scale/crust and allowed a second PDT cycle if there was not a complete response when assessed clinically at three months follow-up. Mosterd 2008 and Abbade 2015 debulked/ shaved tumours under anaesthetic prior to PDT treatments. See Summary of findings 4 and Table 4.

\section{Primary outcomes}

\section{Recurrence}

Although Rhodes 2004 followed-up patients for five years, they presented the per protocol population data and the denominators to be included for our analyses could not be determined for threeyear and five-year recurrence rates. Abbade 2015 showed that at three years, MAL-PDT may increase the risk of recurrence compared to SE $(36.36 \%, 12 / 33$ versus $0 \%, 0 / 35$ ) (RR $26.47,95 \% \mathrm{Cl} 1.63$ to 429.92; Analysis 10.1). We rated the certainty of evidence as low, rating down two levels for very serious imprecision (very wide $95 \% \mathrm{Cl}$ which indicated a slight increase or very large increase in recurrence risk).

Mosterd 2008 showed that ALA-PDT probably increases the risk of recurrence compared to SE at three years $(24.7 \%, 21 / 85$ versus $2.3 \%, 2 / 88$, respectively) (RR $10.87,95 \% \mathrm{Cl} 2.63$ to 44.95 ; Analysis 11.1). By five years, ALA-PDT probably increases the risk of recurrence compared to SE $(27.1 \%, 23 / 85$ versus $2.3 \%$, 2/88, respectively) (RR $11.91,95 \% \mathrm{Cl} 2.90$ to 48.95 ; Analysis 11.2 ). We rated the certainty of evidence for both these outcomes as moderate, rating down for serious imprecision (wide 95\% Cls and only one study).

\section{Cosmetic outcome (participant- and observer-rated)}

Pooling cosmetic results from Szeimies 2008 and Rhodes 2004, we found that when measured at one year, MAL-PDT probably slightly reduces the rate of participant-rated good/excellent cosmetic outcomes compared to SE (RR $1.18,95 \% \mathrm{Cl} 1.09$ to $1.27, \mathrm{I} 2=0 \%$; Analysis 10.2). When investigator-rated at one year, MAL-PDT probably increases the rate of investigator rated good/excellent cosmetic outcomes compared to SE (RR $1.87 .95 \% \mathrm{Cl} 1.54$ to $2.26, \mathrm{I}^{2}$ $=0 \%$; Analysis 10.2). We rated the certainty of evidence as moderate for both these outcomes, rating down for serious risk of bias (unable to truly blind due to nature of interventions).

\section{Secondary outcomes}

\section{Pain during and after treatment}

Rhodes 2004 recorded the frequency of patient reported "pain in skin" and "burning sensation of skin" during treatment and follow-up as part of their AE data. MAL-PDT may result in a higher frequency of pain in the skin than SE $(13.4 \%, 7 / 52$ versus $6.1 \%$, $3 / 49$, respectively) (RR $2.20,95 \% \mathrm{Cl} 0.60$ to 8.03 ; Analysis 10.3 ). We rated the certainty of evidence as low due to the very wide $95 \% \mathrm{Cl}$. Szeimies 2008 reported pain during and after treatment as part of a "photosensitivity reaction" which included all expected reactions to PDT such a "skin discomfort, burning sensation, stinging and erythema". See Table 1 for further pain data from these studies.

\section{Early treatment failure}

At six months follow-up, it is uncertain whether MAL-PDT increases early treatment failures compared to SE as the certainty of evidence is very low due to the very wide $95 \% \mathrm{Cl}$ that meant we rated down three levels for very serious imprecision (RR $11.65,95 \% \mathrm{Cl} 0.67$ to 202.74; Analysis 10.4). Rhodes 2004 and Szeimies 2008 did not histologically confirm their early treatment failures and these data were therefore not extracted.

Mosterd 2008 showed that SE may improve the risk of early treatment failures for nBCCs compared to ALA-PDT (7.2\%, 6/83 versus $2.3 \%, 2 / 88$ ) (RR 3.18, $95 \% \mathrm{Cl} 0.66$ to 15.32; Analysis 11.3). We rated the certainty of evidence as low, rating down for very serious imprecision (very wide $95 \% \mathrm{Cl}$ that indicates important improvement or worsening in early treatment failures).

\section{Adverse effects}

Based on Rhodes 2004, more participants treated with MAL-PDT than SE reported AEs 52\% versus 29\%, ( $P=0.03$, Fisher exact test). Most of the AEs were transient local reactions commonly associated with PDT, such as burning sensation of the skin, pain in the skin or erythema. Szeimies 2008 had similar findings (37\% versus 14\% reported AEs, respectively). Wound infections were reported in three SE participants in Rhodes 2004, one ALA-PDT participant from Mosterd 2008 and one severe wound infection was reported after SE in Szeimies 2008.

See Table 2 for further AE data from the respective studies.

\section{MAL-PDT versus ALA-PDT}

One small pilot study (Kuijpers 2006) compared the topical photosensitisers MAL and ALA in 43 participants with a nBCC ( $<20 \mathrm{~mm}$ diameter) with eight weeks follow-up. Half of the tumours were debulked prior to PDT and conventional treatment protocols were followed (two PDT sessions one week apart). All treated tumours were excised eight weeks following treatment. A second, non-inferiority study (Morton 2018) compared a newer, stable nanoemulsion ALA formulation (BF-200 ALA gel) against MALPDT in 231 participants with $275 \mathrm{sBCC}$ and $\mathrm{nBCC}$ with one year follow-up. Conventional treatment protocols were followed for both arms. A third study (Salmivuori 2019) compared BF-200 ALAPDT, hexylaminolevulinate (HAL)-PDT and MAL-PDT in 95 lesions (sBCC and thin $\mathrm{nBCCS}$ ) with a non-inferiority trial design.

A fourth study (Kessels 2018) compared two-fold ALA-PDT with conventional MAL-PDT in 162 participants with SBCC over a oneyear follow-up. The two-fold group were treated with ALA 20\% ointment with both PDT treatments done on the same day, 
separated by two hours. The MAL-PDT group had the two PDT treatments separated by one week.

\section{Primary outcomes}

\section{Recurrence}

Morton 2018 and Kessels 2018 had only one-year follow-up and Salmivuori 2019 is still ongoing therefore no three- or five-year recurrence rates reported. As one year is closer to our early treatment failure time point we have just reported this.

\section{Cosmetic outcome (participant- and observer-rated)}

In Morton 2018, a blinded investigator assessed cosmetic outcome at one year using a five-point scale (impaired, unsatisfactory, satisfactory, good, very good). Only complete responders were included in the analysis. There is probably little to no difference between BF-200 ALA-PDT $(73.2 \%, 41 / 56)$ and MAL-PDT $(68.4 \%$, $39 / 57$ ) on the rate of good/very good cosmetic outcomes (RR $1.07,95 \% \mathrm{Cl} 0.84$ to 1.36 ; Analysis 12.1 ). We rated the certainty of evidence as moderate, rating down for serious imprecision (wide $95 \% \mathrm{Cl}$ that indicates important improvement and worsening of cosmetic outcome).

Kessels 2018 used two blinded investigators to assess cosmetic outcome on the standard four-point scale. Two-fold ALA-PDT probably slightly increases the rate of good/excellent cosmetic outcomes compared to MAL-PDT (RR $1.19,95 \% \mathrm{Cl} 0.98$ to 1.46 ; Analysis 12.1). We rated the certainty of evidence as moderate, rating down for serious imprecision (wide 95\% Cl). The outcomes from these two studies were not pooled as they used different cosmetic outcome scales.

\section{Secondary outcomes}

\section{Pain during and after treatment}

We pooled the pain data from Morton 2018 and Kuijpers 2006 as they had similar PDT protocols and used the same visual analogue scale (VAS). For both studies, VAS scores were slightly higher during cycle two and mean VAS scores ranged over both cycles from 2.4 to 4.8. During cycle one, there is probably little to no difference between ALA-PDT and MAL-PDT on the risk of pain, measured using VAS (mean difference (MD) $0.56,95 \% \mathrm{Cl}-0.76$ to $1.88, \mathrm{I} 2$ $=57 \%$; Analysis 12.2). We rated the certainty of evidence as low, rating down for serious imprecision due to the fairly wide $95 \% \mathrm{Cl}$ and serious inconsistency as $\mathrm{I}^{2}$ suggests substantial heterogeneity. During cycle two, there is probably little to no difference between ALA-PDT and MAL-PDT on the risk of pain (MD 0.43, 95\% Cl -0.18 to $1.05 ; 1^{2}=0 \%$; Analysis 12.2 ). We rated the certainty of evidence as moderate, rating down for serious imprecision.

Based on Kessels 2018, there is probably little to no difference in numerical pain scores (0-10) between two-fold ALA-PDT and MALPDT during cycle one (MD $-0.37,95 \% \mathrm{Cl}-1.13$ to 0.39 ; Analysis 12.3). For cycle 2, there is probably a slightly higher numerical pain score with twofold ALA-PDT over MAL-PDT (3.36 +/- 2.57 versus $2.48+$ 2.57, respectively) (MD $0.88,95 \% \mathrm{Cl} 0.09$ to 1.67 ; Analysis 12.3). We rated the certainty of evidence as moderate for both these outcomes, rating down for serious imprecision.

See Table 1 for further pain data.

\section{Early treatment failure}

In Kuijpers 2006, at eight weeks there may be little to no difference between MAL-PDT and ALA-PDT on the risk of early treatment failure rates $(28.6 \%, 6 / 21$ versus $27.3 \%, 6 / 22$, respectively) (RR $0.95,95 \% \mathrm{Cl} 0.37$ to 2.49 ; Analysis 12.4 ). We rated the certainty of evidence as low due to very serious imprecision (very wide $95 \% \mathrm{Cl}$ that indicates important benefit and harm). Based on Salmivuori 2019, there may be fewer early treatment failures at 3 months with MAL-PDT compared to BF-200 ALA-PDT (3.2\%, 1/31 versus $9.1 \%, 3 / 33$, respectively) (RR $2.82,95 \% \mathrm{Cl} 0.31$ to 25.68 ; Analysis 13.1). We rated the certainty of evidence as low due to very serious imprecision (very wide $95 \% \mathrm{Cl}$ that indicates important improvement or worsening in early treatment failures). Morton 2018 did not histologically confirm early treatment failures and we have not extracted these data.

Based on Kessels 2018, there may be slightly more early treatment failures with MAL-PDT compared with two-fold ALA-PDT (5.1\%, $4 / 79$ versus $3.8 \%, 3 / 79$, respectively) (RR $0.75,95 \% \mathrm{Cl} 0.17$ to 3.24 ; Analysis 12.4). We rated the certainty of evidence as low due to very serious imprecision (very wide $95 \% \mathrm{Cl}$ that indicates important improvement or worsening in early treatment failures).

\section{Adverse effects}

In Morton 2018, the most frequently reported AEs were mild-tomoderate pain, erythema, pruritus and oedema and frequencies were comparable between the two groups. Two-fold ALA-PDT resulted in more post-treatment AEs compared with MAL-PDT. See Table 2 for further AE data.

\section{HAL-PDT versus MAL-PDT}

\section{Early treatment failure}

Based on Salmivuori 2019 (detailed above), we are uncertain whether there is any difference in the three-month early treatment failure rate between HAL-PDT and MAL-PDT ( $6.5 \%, 2 / 31$ versus $3.2 \%$, $1 / 31$, respectively) because the certainty of evidence was rated as very low due to a very wide $95 \% \mathrm{Cl}$ that meant we rated down three levels for imprecision (RR 2.00, 95\% Cl 0.19 to 20.93; Analysis 14.1).

\section{HAL-PDT versus BF-200 ALA-PDT}

\section{Early treatment failure}

Based on Salmivuori 2019 (detailed above), there may be fewer early treatment failures at three months with HAL-PDT compared to BF-200 ALA-PDT(6.5\%, 2/3 versus $9.1 \%, 3.33$ ) (RR $0.71,95 \% \mathrm{Cl}$ 0.13 to 3.97 ; Analysis 15.1). We rated the certainty of evidence as low (very wide $95 \% \mathrm{Cl}$ that indicates important improvement or worsening in early treatment failures).

\section{Adverse effects}

Severity of post-treatment reactions were visually assessed (on a scale: none/minimal/mild/moderate/severe). HAL-PDT had 12 lesions with a moderate reaction and 13 lesions with a severe reaction. BF-200 ALA-PDT had 13 moderate and 11 severe reactions. MAL-PDT had 13 moderate and nine severe reactions. Overall, there was no significant difference between the three groups $\mathrm{P}=0.49$ ).

There was one treatment-related withdrawal from the trial, as one patient from the MAL group experienced remarkable swelling, oedema, erythema and haematoma in the treatment area. 


\section{PDT versus cryosurgery}

Two studies compared PDT against cryosurgery. One study (BassetSeguin 2008) compared a single session of MAL-PDT against cryosurgery in 118 participants with 219 sBCCs over 5 years followup. A second study (Wang 2001) compared a single session of ALAPDT (against cryosurgery) in 88 participants with $\mathrm{nBCC}$ and $\mathrm{SBCC}$ over 1 year follow-up. The standard European protocol for PDT is two PDT sessions separated by 1 week and therefore the single PDT sessions performed in these studies would no longer be considered the standard of care. See Table 5.

\section{Primary outcomes}

\section{Recurrence}

There may be little to no difference between MAL-PDT and cryosurgery on the risk of recurrence at three years (22\%, 22/100 versus $19.4 \%, 18 / 93$ ) (RR 1.14, 95\% Cl 0.65 to 1.98 ; Analysis 16.1). We rated the certainty of evidence as low, rating down for very serious imprecision (very wide $95 \% \mathrm{Cl}$ that indicates important benefit and harm).

At five years there may be little to no difference between MAL-PDT and cryosurgery on the risk of recurrence (22\%, 22/100 versus $20 \%$, $19 / 93$, respectively) (RR $1.08,95 \% \mathrm{Cl} 0.62$ to 1.86 ; Analysis 16.2). We rated the certainty of evidence as low for very serious imprecision (very wide $95 \% \mathrm{Cl}$ that indicates important benefit and harm).

\section{Cosmetic outcome (participant- and observer-rated)}

When participant-rated at one year on a four-point scale, MALPDT probably increases the rate of good/excellent cosmetic outcomes compared to cryosurgery $(100 \%, 51 / 51$ versus $81.3 \%$, 39/48, respectively) (RR $1.23,95 \% \mathrm{Cl} 1.07$ to 1.41 ; Analysis 16.3). When investigator-rated on a four-point scale, MAL-PDT probably increases the rate of good/excellent cosmetic outcomes compared to the cryosurgery $(89 \%, 45 / 51$ versus $61 \%, 29 / 48$, respectively) (RR 1.46, 95\% Cl 1.14 to 1.88; Analysis 16.3). Wang 2001 assessed cosmetic outcome based on hypo- and hyperpigmentation, scar and tissue defect and then gave an overall investigator rated assessment based on a four-point scale. The study showed that ALA-PDT probably increases the rate of good/excellent cosmetic outcomes at one year compared to cryosurgery (92.8\%, 39/42 versus $54.1 \%, 20 / 37$ ) (RR 1.72, 95\% Cl 1.26 to 2.34; Analysis 17.1). We rated the certainty of evidence as moderate for all these outcomes, rating down for serious risk of blinding bias due to the nature of the interventions.

\section{Secondary outcomes}

\section{Pain during and after treatment}

Basset-Seguin 2008 presented pain data within the adverse effects data. There may be little to no difference between MAL-PDT and cryosurgery on the risk of pain reported as an AE (33\%, 19/58 versus $37 \%, 22 / 60$, respectively) (RR $1.12,95 \% \mathrm{Cl} 0.68$ to 1.84 ; Analysis 16.4). We rated the certainty of evidence as low (very wide $95 \% \mathrm{Cl}$ that indicates important benefit and harm). Wang 2001 measured pain during and the first week after treatment by providing participants with a self-registration VAS form which ranged from $0 \mathrm{~mm}$ (no pain) to $115 \mathrm{~mm}$ (unbearable pain). Raw data with standard deviations were available for the mean during treatment VAS scores which showed that ALA-PDT probably results in slightly more pain than cryosurgery (MD $11.00,95 \% \mathrm{Cl}-1.12$ to 23.12; Analysis 17.2). We rated the certainty of evidence as moderate rating down for serious imprecision (only one study and wide $95 \% \mathrm{Cl}$ ). In the first week after treatment,

See Table 1 for further pain data.

\section{Early treatment failure}

At three months, there may be slightly fewer early treatment failures with MAL-PDT compared to cryosurgery $(2.9 \%, 3 / 103$ versus $5.1 \%, 5 / 98$, respectively) (RR $0.57,95 \% \mathrm{Cl} 0.57$ to 2.33 ; Analysis 16.5). We rated the certainty of evidence as low, rating down for very serious imprecision (very wide $95 \% \mathrm{Cl}$ that indicates important benefit and harm).

\section{Adverse effects}

See Table 2 for further AE data.

\section{Imiquimod cream versus MAL-PDT}

One study (Arits 2013) assessed whether imiquimod cream and 5FU cream were non-inferior to MAL-PDT in 601 participants with a single SBCC (anywhere except high-risk face/scalp) in a three-arm RCT with five years follow-up. A pre-specified non-inferiority margin of $10 \%$ was used. See Summary of findings 5 .

\section{Primary outcomes}

\section{Recurrence}

Imiquimod cream probably reduces the risk of recurrence at three years compared to MAL-PDT $(22.8 \%, 34 / 149$ versus $51.6 \%, 66 / 128$, respectively) (RR $0.44,95 \% \mathrm{Cl} 0.32$ to 0.62 ; Analysis 18.1 ). We rated the certainty of evidence as moderate, rating down for serious imprecision as this was a single study.

At five years, imiquimod cream probably reduces the risk of recurrence compared to MAL-PDT $(28.6 \%, 36 / 126$ versus $68.6 \%$, 70/102) (RR 0.42, 95\% Cl 0.31 to 0.57 ; Analysis 18.2). We rated the certainty of evidence as moderate, rating down for serious imprecision (only one study).

\section{Cosmetic outcome (participant- and observer-rated)}

A blinded observer-rated cosmetic outcome at one year on a four-point scale. There is probably little to no difference between imiquimod and MAL-PDT on the rate of good/excellent cosmetic outcomes (RR $0.98,95 \% \mathrm{Cl} 0.84$ to 1.16; Analysis 18.3). We rated the certainty of evidence as moderate (only one study).

\section{Secondary outcomes}

\section{Pain during and after treatment}

Maximum pain was recorded on VAS for all interventions at the end of each week of treatment. The scores were then interpreted as absent/mild (0-3), moderate (4-6) and severe (7-10). We selected the week or treatment cycle (presented for PDT only) with the highest reported moderate/severe pain to overcome differences in timing of reported pain with the different interventions. Week six had the highest reported pain for imiquimod (18\% of patients reported moderate/severe pain) and treatment cycle two for MALPDT ( $31 \%$ of patients reporting moderate/severe pain). Our analysis showed that imiquimod probably reduces the risk of moderate/ severe pain compared to MAL-PDT (RR $0.60,95 \% \mathrm{Cl} 0.41$ to 0.87 ; Analysis 18.4). We rated the certainty of evidence as moderate, rating down for serious imprecision (only one study). See Table 1 for further pain data from this study. 


\section{Early treatment failure}

At three months, there may be little to no difference between imiquimod and MAL-PDT on the risk of early treatment failures (10.1\%, 19/189 versus $15.8 \%, 31 / 196)$ (RR $0.64,95 \% \mathrm{Cl} 0.37$ to 1.09 ; Analysis 18.5). We rated the certainty of evidence as moderate, rating down for serious imprecision (wide $95 \% \mathrm{Cl}$, including the null effect which indicates there may be an important difference or no difference between the two treatments).

\section{Adverse effects}

Participants treated with imiquimod more often reported moderate to severe local swelling, erosion, crust formation and itching of skin compared to the MAL-PDT group. There were no unexpected serious adverse reactions in the MAL-PDT group, but $4.8 \%$ (nine participants) were reported to have a suspected unexpected serious adverse reaction (SUSAR) in the imiquimod group for influenza-like symptoms $(n=8)$ and a local wound infection; however, none of the participants were admitted to hospital. See Table 2 for further AE data from this study.

\section{5-fluorouracil cream versus MAL-PDT}

One study (Arits 2013) assessed the effects of 5-FU cream versus MAL-PDT; this study is described above in section 5. See Table 6.

\section{Primary outcomes}

\section{Recurrence}

5-FU cream probably reduces the risk of recurrence compared to MAL-PDT at three years (34.2\%, 50/146 versus $52.4 \%, 66 / 126$, respectively) (RR $0.65,95 \% \mathrm{Cl} 0.49$ to 0.86 ; Analysis 19.1 ). We rated the certainty of evidence as moderate, rating down for serious imprecision (only one study).

Similar findings were also seen at five years, where 5-FU cream probably reduces the risk of recurrence compared to MAL-PDT (46\%, 57/124 versus $68.7 \%, 70 / 102$, respectively) (RR $0.67,95 \% \mathrm{Cl}$ 0.53 to 0.84 ; Analysis 19.2). We rated the certainty of evidence as moderate, rating down for serious imprecision (only one study).

\section{Cosmetic outcome (participant- and observer-rated)}

There is probably little to no difference between 5-FU cream and MAL-PDT on the rate of good/excellent cosmetic outcomes (RR $0.92,95 \% \mathrm{Cl} 0.78$ to 1.09 ; Analysis 19.3 ). We rated the certainty of evidence as moderate, rating down for serious imprecision (only one study).

\section{Secondary outcomes}

\section{Pain during and after treatment}

The maximum recorded pain for 5-FU cream was recorded during week four (final week) of treatment with $12 \%$ of patients reporting moderate/severe pain. 5-FU cream probably reduces the risk of moderate/severe pain compared to MAL-PDT (RR $0.41,95 \% \mathrm{Cl}$ 0.26 to 0.63 ; Analysis 19.4). We rated the certainty of evidence as moderate, rating down for serious imprecision (only one study). See Table 1 for further pain data.

\section{Early treatment failure}

There may be little to no difference between 5-FU cream and MALPDT on the risk of early treatment failure $(12.1 \%, 24 / 198$ versus $15.8 \%, 31 / 196)$ (RR $0.77,95 \% \mathrm{Cl} 0.47$ to 1.26 ; Analysis 19.5). We rated the certainty of evidence as low, rating down two levels for very serious imprecision (very wide $95 \% \mathrm{Cl}$ that indicates important benefit and harm).

\section{Adverse effects}

Unexpected serious adverse reactions were reported in 2.1\% (four participants) in the 5-FU cream group: two local wound infections, one case of erysipelas of the leg and one leg ulcer. See Table 2 for further AE data.

\section{Laser ALA-PDT versus broadband halogen light ALA-PDT}

One study (Soler 2000) of 83 participants (245 lesions) with sBCC compared two different light sources: laser or broadband lamp light with ALA-PDT. The study only had six months follow-up. The laser light was $630 \mathrm{~nm}$ from a copper vapour dye laser with doses ranging between $100 \mathrm{~J} / \mathrm{cm}^{2}$ to $150 \mathrm{~J} / \mathrm{cm}^{2}$. The broadband light used a halogen bulb and a filter to produce red light $(570 \mathrm{~nm}$ to $740 \mathrm{~nm})$ with a dose ranging between $150 \mathrm{~J} / \mathrm{cm}^{2}$ to $200 \mathrm{~J} / \mathrm{cm}^{2}$ as well as infrared radiation ( $1180 \mathrm{~nm}$ to $2700 \mathrm{~nm}$ ).

\section{Primary outcomes}

\section{Cosmetic outcome (participant- and observer-rated)}

At six months, cosmetic outcome was rated by two observers (only one blinded) on a four-point scale (poor, fair, good, excellent). Only tumours that had completely responded were scored leading to an unequal attrition rate between the groups. There may be little to no difference on the rate of good/excellent cosmetic outcomes between the two light sources ( $\mathrm{RR} 0.91,95 \% \mathrm{Cl} 0.82$ to 1.01; Analysis 20.1). We rated the certainty of evidence as low, rating down for serious imprecision (only one study) and serious risk of attrition bias.

\section{Secondary outcomes}

\section{Pain during and after treatment}

There is probably little to no difference between laser ALA-PDT and broadband light ALA-PDT on the frequency of pain (reported as any type of discomfort) during and immediately after treatment $(83 \%$, $34 / 41$ versus $76 \%, 32 / 42$ ) (RR $1.09,95 \% \mathrm{Cl} 0.87$ to 1.35; Analysis 20.2). In the first week after treatment, there is probably little to no difference in the frequency of pain reported between the two light sources (RR 0.93, 95\% Cl 0.70 to 1.22; Analysis 20.2). We rated the certainty of evidence for both these outcomes as moderate, rating down for serious imprecision (only one study). See Table 1 for further pain data from this study.

\section{Early treatment failure}

The study did not histologically confirm clinical treatment failures; therefore, we have not extracted the data for this outcome.

\section{Adverse effects}

Some discomfort during and after illumination was reported by $83 \%$ of participants receiving laser ALA-PDT and $76 \%$ of those receiving broadband PDT. No serious AEs were reported during the 6-month follow-up. See Table 2 for further AE data from this study.

\section{Ablative fractional laser (AFXL) MAL-PDT versus MAL-PDT}

Two small pilot studies compared ablative fractional laser (AFXL) pre-treatment prior to MAL-PDT against conventional MAL-PDT with one year follow-up. This is based on the rationale that AFXL pre- 
treatment increases uptake of MAL deeper into the tumour. One study (Haak 2015) randomised 32 participants with facial nBCC and the other (Choi 2016) randomised 39 participants (42 lesions) with facial $\mathrm{nBCC}$. The first study used a fractional $\mathrm{CO}_{2}$ laser and the second an erbium:YAG laser.

\section{Primary outcome}

\section{Cosmetic outcome (participant- and observer-rated)}

Cosmetic outcomes were assessed by a blinded investigator in both studies at one year using a four-point scale. The pooled results from both studies showed there may be little to no difference between AFXL-PDT and MAL-PDT on the rate of good/excellent cosmetic outcomes (RR 1.12, 95\% Cl 0.54 to $2.31, \mathrm{I}^{2}=66 \%$; Analysis 21.1). We rated the certainty of evidence as low, rating down two levels for very serious imprecision (very wide $95 \% \mathrm{Cl}$ that indicates important benefit and harm). Haak 2015 presented participant reported cosmetic outcomes, showing that there is probably little to no difference between AFXL-PDT and MAL-PDT on the rate of good/excellent cosmetic results (RR $0.94,95 \% \mathrm{Cl} 0.75$ to 1.17 ; Analysis 21.1). We rated the certainty of evidence as moderate, rating down for serious imprecision (only one study).

\section{Secondary outcomes}

\section{Pain during and after treatment}

Pain was recorded using VAS for both studies but individual data were not presented. Both studies reported in their text that there was "no statistically significant difference" in pain scores between the treatment groups. See Table 1 for further pain data from these studies.

\section{Early treatment failure}

A pooled analysis of both studies showed that AFXL-PDT probably reduces the risk of early treatment failures compared to MAL-PDT at three months (RR $0.38,95 \% \mathrm{Cl} 0.17$ to $0.84, \mathrm{I}^{2}=0 \%$; Analysis 21.2 ). We rated the certainty of evidence as moderate, rating down for serious imprecision (fairly wide $95 \% \mathrm{Cl}$ ).

\section{Adverse effects}

See Table 2 for further AE data from these studies.

\section{MAL-PDT versus placebo}

Two RCTs with the "same design and procedures" (Foley 2009a and Foley 2009b) randomised 131 participants (160 nBCC) to MALPDT versus placebo. We have presented the data analyses as one study under Foley 2009a as the data from the separate studies were not available. All tumours were debulked prior to treatment and all treatment sites were excised at three months (clinical non-responders) or six months (clinical responders) after the last treatment.

\section{Primary outcomes}

\section{Cosmetic outcome (participant- and observer-rated)}

Only the complete clinically/histologically clear lesions were assessed for cosmetic outcome. The authors only presented the investigator-rated data (four-point scale). There is probably little to no difference between MAL-PDT and placebo on the rate of good/ excellent cosmetic outcomes (RR 1.05, 95\% Cl 0.91 to 1.21 ; Analysis 22.1). We rated the certainty of evidence as moderate, rating down for serious imprecision (fairly wide $95 \% \mathrm{Cl}$ ).

\section{Secondary outcomes}

\section{Pain during and after treatment}

Frequency of "burning sensation of skin", "pain in skin" and "stinging of skin" were reported as part of treatment-related local adverse effects. MAL-PDT probably increases the risk of "pain in skin" compared to placebo (RR 3.94, $95 \% \mathrm{Cl} 1.17$ to 13.32; Analysis 22.2). We rated the certainty of evidence as moderate, rating down for serious imprecision (fairly wide $95 \% \mathrm{Cl}$ ). See Table 1 for further pain data from this study.

\section{Early treatment failure}

MAL-PDT may reduce the risk of early treatment failures compared to placebo (RR $0.36,95 \% \mathrm{Cl} 0.24$ to 0.54 ; Analysis 22.3 ). We rated the certainty of evidence as low, rating down for serious imprecision (only one study).

\section{Adverse effects}

See Table 2 for further AE data from this study.

\section{Imiquimod cream \\ Imiquimod cream versus 5-fluorouracil cream}

One study (Arits 2013) assessed the effects of imiquimod cream versus 5-FU cream; this study is described above in section 5 . See Table 7.

\section{Primary outcomes}

\section{Recurrence}

Imiquimod probably reduces the risk of recurrence compared to 5 -FU cream at three years $(23.4 \%, 34 / 145$ versus $34.2 \%, 50 / 146$, respectively) (RR $0.68,95 \% \mathrm{Cl} 0.47$ to 0.99 ; Analysis 23.1 ). We rated the certainty of evidence as moderate, rating down for serious imprecision (only one study).

Imiquimod probably reduces the risk of recurrence compared to 5 -FU cream at five years $(28.6 \%, 36 / 126$ versus $46 \%, 57 / 124$, respectively) (RR $0.62,95 \% \mathrm{Cl} 0.44$ to 0.87 ; Analysis 23.2 ). We rated the certainty of evidence as moderate, rating down for serious imprecision (only one study).

\section{Cosmetic outcome (participant- and observer-rated)}

When blinded observer-rated at 1 year, there is probably little to no difference between imiquimod and 5-FU cream on the rate of good/excellent cosmetic outcomes $(61.4 \%, 113 / 184$ versus $57.5 \%$, $111 / 193$, respectively) (RR $1.07,95 \%$ Cl 0.90 to 1.26 ; Analysis 23.3). We rated the certainty of evidence as moderate, rating down for serious imprecision (only one study).

\section{Secondary outcomes}

\section{Pain during and after treatment}

We compared the week with the highest frequency of reported of moderate/severe pain during treatment for each intervention (week six for imiquimod and week 4 for 5 -FU cream). There is probably a slightly increased risk of moderate/severe pain with imiquimod compared with 5 -FU cream (18.2\%, 33/181 versus $12.5 \%, 23 / 184$ ) (RR 1.46, $95 \% \mathrm{Cl} 0.89$ to 2.34; Analysis 23.4). We rated the certainty of evidence as moderate, rating down for serious imprecision (wide $95 \% \mathrm{Cl}$ that indicates increased pain or slightly less pain). See Table 1 for further pain data from this study. 


\section{Early treatment failure}

There may be little to no difference between imiquimod and 5-FU cream on the risk of early treatment failures (10\%, 19/189 versus $12.1 \%, 24 / 198$ ) (RR $0.83,95 \% \mathrm{Cl} 0.47$ to 1.46 ; Analysis 23.5). The certainty of evidence is low due to very serious imprecision with a wide $95 \% \mathrm{Cl}$ that indicates there may be an important reduction or an important increase in early treatment failures.

\section{Adverse effects}

See Table 2 for further AE data from this study.

\section{Imiquimod cream versus radiotherapy}

One small study (Garcia-Martin 2011) randomised 27 participants with $\mathrm{nBCC}$ of the eyelid to either imiquimod (five days a week for six weeks) or conventional radiotherapy with two years follow-up. The study was at high risk of selection bias due to the method of randomisation (based on birthday of participant).

\section{Primary outcomes}

\section{Recurrence}

There were no recurrences in either group during the follow-up period and therefore we were unable to assess effect size.

\section{Cosmetic outcome (participant- and observer-rated)}

Cosmesis was patient-rated on a three-point scale (bad, good, excellent), but this was only measured at 6 weeks following treatment. Observer-rated cosmetic outcomes were also recorded on a three-point scale, but the data were not presented in the paper. All participants in the imiquimod group rated the cosmetic result as excellent and all those in the radiotherapy group rated the cosmetic result as good. There may be more participant-rated excellent cosmetic outcomes at 6 weeks with imiquimod compared to radiotherapy (RR 25.19, 95\% Cl 1.66 to 382.13; Analysis 24.1). We rated the certainty of evidence as low due to the very wide $95 \% \mathrm{CI}$.

\section{Secondary outcomes}

\section{Pain during and after treatment}

Blink discomfort was reported in $60 \%$ (9/15) of participants in the imiquimod group and blink discomfort and dry eye were reported in $50 \%(6 / 12)$ participants in the radiotherapy group. Intense conjunctival irritation occurred in $13.3 \%$ (2/15) of participants in the imiquimod group.

\section{Early treatment failure}

There were no early treatment failures at six weeks following treatment and therefore no effect size was estimable.

\section{Adverse effects}

All participants in the radiotherapy group presented with loss of eyelashes in one or both eyelids, $16.6 \%$ (2/12) of participants had inferior eyelid retraction with secondary ectropion, and three of the participants reported dry eye symptoms after radiotherapy. Tolerability during treatment was better in the radiotherapy group. Seven of the 15 participants in the imiquimod group reported 'bad' tolerability, but these symptoms disappeared when the period of cream application ended. Only one patient in the radiotherapy group rated 'bad' tolerability. See Table 2 for further AE data and Table 1 for pain data from this study.

\section{Imiquimod with occlusion versus imiquimod without occlusion}

Two similar dose-response studies ( Sterry 2002a; Sterry 2002b) examined imiquimod cream with or without occlusion in SBCC (93 participants; Sterry 2002a) and nBCC (90 participants; Sterry $2002 b)$. Patients were randomised to one of four groups to apply imiquimod 5\% cream two or three days per week either with or without occlusion for six weeks. At the end of the six-week treatment period, the entire target tumour area was excised and histologically examined for evidence of residual tumour. Size and location of lesions were not given.

\section{Secondary outcomes}

\section{Early treatment failure}

Pooling the data from both studies (we also combined the different dosing regimen group as the number of participants was small) found there may be little to no difference between the occlusion and no occlusion groups (38.2\%, $34 / 89$ versus $41.5 \%, 39 / 94)$ on the risk of early treatment failures (RR $0.91,95 \% \mathrm{Cl} 0.64$ to 1.33 ; Analysis $25.1,12=0 \%$ ). We rated the certainty of evidence as low due to very serious imprecision (wide $95 \% \mathrm{Cl}$ that indicates that includes the possibility of important benefit or harm).

\section{Adverse effects}

See Table 2 for further AE data from these studies.

\section{Imiquimod versus placebo}

One trial (Beutner 1999) randomised 35 participants with SBCC and $\mathrm{nBCC}$ to imiquimod cream or vehicle cream in one of five dosing regimens for up to 16 weeks. Another trial of 128 participants (Geisse 2002) compared imiquimod twice daily, once daily, five days per week or three days per week versus vehicle for sBCCs. Marks 2001 compared different dosages of imiquimod cream applied for six weeks in 99 Australian participants with primary sBCCs. Geisse 2004 randomised 724 participants with SBCC to imiquimod cream daily either five times per week or seven times per week for six weeks versus vehicle. One further study (Schulze 2005), randomised 166 participants with sBCC to imiquimod cream or vehicle, once a day for six weeks. Another two similar studies (Shumack 2002a; Shumack 2002b) randomised 99 and 92 participants with $\mathrm{nBCC}$, respectively to four different dosing regimens (three or seven days per week or twice daily for three or seven days per week) of imiquimod cream or vehicle over 12 and six weeks, respectively. All these studies were industry sponsored.

\section{Early treatment failure}

Pooled analysis of the five studies showed that imiquimod reduces the risk of early treatment failures compared to vehicle by $75 \%$ (RR $0.25,95 \% \mathrm{Cl} 0.19$ to $0.32, \mathrm{I}^{2}=57 \%$; Analysis 26.1 ). We rated the certainty of evidence as high.

\section{Dose-ranging studies}

One study (Ezughah 2008) compared the tolerability of two regimens of imiquimod for the treatment of SBCC with one year follow-up: five weeks of once-daily dosing with a one-week interval in the middle of the course (R1) versus eight weeks of treatment with once-daily dosing for alternate weeks (R2). One study (Eigentler 2007) compared imiquimod three times a week over eight weeks versus three times a week over 12 weeks in 102 
participants with $\mathrm{nBCC}$ up to $1.5 \mathrm{~cm}$, located anywhere on the body (except close to the eyes).

\section{Primary outcomes}

\section{Recurrence}

Ezughah 2008 only followed up patients for one year and as we have not included the early treatment failure data (as not histologically confirmed) we have included these data. R2 imiquimod dosing may result in fewer recurrences than $\mathrm{R} 1$ imiquimod dosing $(12.5 \%, 2 / 16$ versus $57.1 \%, 8 / 14$, respectively) (RR $4.57,95 \% \mathrm{Cl} 1.16$ to 18.05 ; Analysis 27.1). We rated the certainty of evidence as moderate, rating down for serious imprecision due to the wide $95 \% \mathrm{Cl}$ and the small numbers of participants.

\section{Secondary outcomes}

\section{Early treatment failure}

Based on Eigentler 2007, at eight weeks post-treatment there may be little to no difference in early treatment failure rates between imiquimod three times a week over eight weeks and three times a week over 12 weeks $(35.6 \%, 16 / 45$ versus $37.8 \%, 17 / 45)$ (RR $0.94,95 \% \mathrm{Cl} 0.55$ to 1.62 ; Analysis 28.1 ). We rated the certainty of evidence as low, rating down two levels for very serious imprecision (very wide $95 \% \mathrm{Cl}$ that indicates important benefit and harm).

\section{Pain during and after treatment}

In Ezughah 2008, a weekly VAS for tolerability was measured using a $10 \mathrm{~cm}$ line scale ranging from $0 \mathrm{~cm}$ (not unpleasant) to 10 $\mathrm{cm}$ (absolutely intolerable). There may be little to no difference between the eight-week and five-week treatment groups on the mean composite VAS scores at eight weeks (median score $0.85 \mathrm{~cm}$ versus median score $0.30 \mathrm{~cm} ; 95 \% \mathrm{Cl}$ for difference in medians: 20.40 to $0.9 \mathrm{~cm}, \mathrm{P}=0.39$ ), as reported in the paper. Eigentler 2007 did not present pain data separately for the two treatment arms. See Table 1 for further pain data from these studies.

\section{Adverse effects}

See Table 2 for AE data from these studies.

\section{5-Fluorouracil}

\section{Phosphatidyl choline versus petrolatum vehicle}

One small pilot study (Romagosa 2000) compared 5-FU cream in petrolatum with 5-FU cream in phosphatidyl choline (a transepidermal carrier to aid penetration) in 13 participants (17 lesions, non SBCC, at least $0.7 \mathrm{~cm}$ diameter, facial lesions excluded). Participants underwent excision of the treated BCC site at week-16.

\section{Secondary outcomes}

\section{Early treatment failure}

There may be fewer early treatment failures with 5-FU cream in phosphatidyl choline compared to 5-FU cream in petrolatum $(10 \%$, $1 / 0$ versus $42.9 \%, 3 / 7$, respectively) (RR $0.23,95 \% \mathrm{Cl} 0.03$ to 1.81 ; Analysis 29.1). We rated the certainty of evidence as low, rating down two levels for very serious imprecision (very wide $95 \% \mathrm{Cl}$ that indicates important benefit and harm).

\section{Adverse effects}

In the Romagosa 2000 study, local irritation, erythema, ulceration and tenderness were common reactions but were reported to be well-tolerated by the participants. Minimal itching and discomfort were experienced by some of the participants in both treatment arms. See Table 2 for further AE data from this study.

\section{Intralesional 5-fluorouracil/epinephrine gel}

One study (Miller 1997) compared six treatment regimens of intralesional 5-FU/epinephrine gel in 122 participants with SBCC and $\mathrm{nBCC}$ located on the head, upper and lower extremities: $1 \mathrm{~mL}$ versus $0.5 \mathrm{~mL}$ both once a week for six weeks; $1 \mathrm{~mL}$ versus $0.5 \mathrm{~mL}$ both twice a week for three weeks; $0.5 \mathrm{~mL}$ twice a week for four weeks versus $0.5 \mathrm{~mL}$ three times a week for two weeks.

\section{Early treatment failure}

There may be fewer early treatment failures with $1 \mathrm{~mL}$ intralesional 5-FU compared with $0.5 \mathrm{~mL}$ intralesional 5-FU when given twice a week for three weeks (5.6\%, $1 / 18$ versus $21.1 \%, 4 / 19$, respectively) (RR $0.26,95 \% \mathrm{Cl} 0.03$ to 2.14; Analysis 31.1 ). We rated the certainty of evidence as low, rating down two levels for very serious imprecision (very wide $95 \% \mathrm{Cl}$ that indicates important benefit and harm). It is uncertain whether there is any difference between the other two intralesional regimens as we rated the certainty of evidence for these comparisons as very low due, rating down three levels for very serious imprecision (Analysis 30.1; Analysis 32.1).

\section{Adverse effects}

All participants had transient, moderate to severe stinging, burning or pain at the time of injection. Local tissue reactions were confined to the treatment site and included erythema, swelling, desquamation, erosions and eschar in most participants. See Table 2 for further AE data from this study.

\section{Electrochemotherapy}

\section{Electrochemotherapy versus surgical excision}

One study (Clover 2016) compared bleomycin electrochemotherapy (ECT) to SE in 100 participants with 117 low-risk and high-risk BCC (superficial, nodular, infiltrative and morphoeic subtypes). Noduar and nodular/infiltrative BCC were the most common subtype. Location of lesions was not described. Follow-up was for five years. The author of the abstract was contacted and unpublished data were obtained.

\section{Primary outcomes}

\section{Recurrence}

ECT may result in more recurrences $(10.5 \%, 4 / 38$ versus $2.8 \%, 1 / 36$, respectively) than SE at three years (RR $3.79,95 \% \mathrm{Cl} 0.44$ to 32.32 ; Analysis 33.1 ) and 5 years $(13.5 \%, 5 / 37$ versus $2.8 \%, 1 / 36$ ) (RR $4.86,95 \% \mathrm{Cl} 0.60$ to 39.63 ; Analysis 33.2 ). We rated the certainty of evidence for both these outcomes as low rating down two levels for the very wide $95 \% \mathrm{Cl}$ that includes the possibility of important benefit or harm.

\section{Cosmetic outcome (participant- and observer-rated)}

When assessed with the Vancouver scar scale by four blinded plastic surgeons 18 months after treatment, the authors reported there was no significant difference between ECT and SE; however the raw data were not available for further analysis. 


\section{Ingenol mebutate}

\section{Day 1 and 2 versus day 1 and 8 regimens}

One study (Siller 2010) randomised 60 participants with SBCC to one of two different treatment regimens (days 1 and 2 dosing or days 1 and 8 dosing) and then randomised again within each group to treatment with one of three different concentrations of ingenol mebutate gel $(0.0025 \%, 0.01 \%$ and $0.05 \%)$, or vehicle. The main outcome measures were the incidence and severity of adverse effects and local skin responses.

\section{Secondary outcomes}

\section{Pain during and after treatment}

Intensity of pain data were not presented. Within the adverse effects data, only two patients reported application site pain and these were in the highest dose ingenol group $(0.05 \%)$ in the days 1 and 8 regimen. See Table 1 for further pain data from this study.

\section{Early treatment failure}

At week-12, in the day 1 and 2 dosing group, ingenol $0.05 \%$ gel probably results in fewer early treatment failures than vehicle gel (37.5\%, 3/8 versus 100\%, 6/6, respectively) (RR $0.42,95 \% \mathrm{Cl}$ 0.18 to 0.97 ; Analysis 34.1 ). We rated the certainty of evidence as moderate, rating down for serious imprecision. For the lower ingenol concentration in the day 1 and 2 dosing group, as well as all concentrations in the day 1 and 8 dosing group, there may be little to no difference between ingenol and vehicle (see analysis Analysis 35.1 and Analysis 36.1 for RRs). We rated the certainty of evidence as low for both these outcomes, rating down for serious imprecision due to the wide $95 \% \mathrm{Cls}$ and low numbers of participants involved.

\section{Adverse effects}

See Table 2 for further AE data from this study.

\section{Occlusion versus no occlusion}

One study (Spelman 2014) compared ingenol mebutate $0.05 \%$ gel given on three consecutive days with full occlusion (aluminium disk dressing) versus semi-occlusion (Opsite ${ }^{\mathrm{TM}} \mathrm{Disk}$ ) and no occlusion in 75 participants with SBCC ( $4 \mathrm{~mm}-15 \mathrm{~mm}$ to diameter) on the trunk/ extremities in a phase two RCT with four months follow-up.

\section{Secondary outcomes}

\section{Early treatment failure}

At four months, ingenol with full occlusion may reduce the risk of early treatment failures compared to no occlusion $(29.6 \%, 8 / 27$ versus $45.5 \%, 11 / 24$, respectively) (RR $0.65,95 \% \mathrm{Cl} 0.31$ to 1.34 ; Analysis 36.1), and semi-occlusion may increase the risk of early treatment failures compared to no occlusion $(62.5 \%, 15 / 24$ versus $45.8 \%, 11 / 24$, respectively) (RR $1.36,95 \% \mathrm{Cl} 0.80$ to 2.33 ; Analysis 36.1). We rated the certainty of evidence as low for both these outcomes, rating down for serious imprecision due to the wide $95 \%$ $\mathrm{Cl}$ and low numbers of participants involved.

\section{Adverse effects}

The percentage of participants with AEs were similar across all three treatment groups. The most common AE was application site pain. See Table 2 for further AE data from this study.

\section{Solasodine glycosides}

One study (Punjabi 2008) of 94 participants compared solasodine glycoside (Zycure) cream to matching vehicle in BCCs at least $0.5 \mathrm{~cm}$ diameter (excluding morphoeic BCC). Lesions were treated twice daily under occlusion with solasodine glycoside cream or vehicle for 8 weeks.

\section{Primary outcomes}

Cosmetic outcome (participant- and observer-rated)

Cosmetic outcome was evaluated by an assessment of scarring during the follow-up (categorized as none, mild, moderate, severe). The authors reported there was no statistically significant difference between the treatment groups; however, raw data were not presented for further analysis.

\section{Secondary outcomes}

\section{Early treatment failure}

At the end of 8 weeks of treatment, a $2 \mathrm{~mm}$ punch biopsy was performed on all lesions. Solasodine glycoside cream probably reduces the risk of early treatment failures compared to vehicle (33.9\%, 21/62 versus 75\%, 24/32, respectively) (RR $0.45,95 \% \mathrm{Cl}$ 0.30 to 0.67 ; Analysis 37.1 ). We rated the certainty of evidence as moderate, rating down for serious imprecision (only one study).

\section{Adverse effects}

No major treatment-related adverse effects were reported. See Table 2 for further AE data from this study.

\section{Topical valproic acid and tazarotene}

One study (2005-001474-27) randomised 50 participants (subtype not stated, located anywhere except eyelids, nose, lips, anogenital) to topical valproic acid (Baceca ${ }^{\circledR} 3 \%$ gel) and tazarotene (Zorac ${ }^{\circledR}$ $0.1 \%$ gel) against placebo gel and tazarotene. Treatment lasted eight weeks and involved applying valproic acid or placebo gel before bedtime and 15 to 30 minutes later tazarotene was applied. Participants in both groups that did not show a complete clearance after eight weeks, completed a further eight weeks of treatment with valproic acid and tazarotene (cross-over design).

\section{Early treatment failure}

At three months follow-up, there may be slightly fewer early treatment failures in the valproic acid gel and tazarotene group compared with the placebo gel and tazarotene group (31\%, 8/25 versus $52 \%, 13 / 25$, respectively) (RR $0.62,95 \% \mathrm{Cl} 0.31$ to 1.22 ; Analysis 38.1 ). We rated the certainty of evidence as low, rating down one level for serious imprecision ( wide $95 \% \mathrm{Cl}$ that indicates important benefit and slight harm).

\section{Adverse effects}

Erythema, itching, ulceration and localised pain were the most frequently reported $A E s$ across both groups. There were no drugrelated severe AEs. See Table 2 for further AE data from this study.

\section{Topical diclofenac gel and calcitriol ointment (vitamin D)}

One study (Brinkhuizen 2016) of 128 participants (64 sBCC and 64 $\mathrm{nBCC}$ ) compared diclofenac $3 \%$ gel (Solaraze ${ }^{\mathrm{TM}}$ ), calcitriol $3 \mu \mathrm{g} / \mathrm{g}$ ointment (Silkis $\left.{ }^{\circledast}\right)$, a combination of both or no topical treatment. The active treatments were applied twice daily under occlusion for 
eight weeks. We have not included the control arm comparisons in our analyses.

\section{Secondary outcomes}

\section{Early treatment failure}

Early treatment failure was measured at eight weeks of treatment. Diclofenac gel monotherapy may reduce the risk of early treatment failures compared to calcitriol ointment $(53.3 \%, 16 / 30$ versus $97 \%$, $31 / 32$, respectively) (RR of $0.55,95 \% \mathrm{Cl} 0.39$ to 0.77 ; Analysis 39.1 ). We rated the certainty of evidence as moderate, rating down for serious imprecision (only one study).

There may be little to no difference between diclofenac gel monotherapy and combination treatment of diclofenac and calcitriol on the risk of early treatment failures $(53.3 \%, 16 / 30$ versus $61.3 \%, 19 / 31$, respectively) (RR $0.87,95 \% \mathrm{Cl} 0.56$ to 1.35 ; Analysis 40.1). We rated the certainty of evidence as low, rating down for very serious imprecision due to the $95 \% \mathrm{Cl}$ that indicates important benefit and harm.

Calcitriol ointment may increase the risk of early treatment failures compared to combination treatment of diclofenac and calcitriol (97\%, 31/32 versus $61.3 \%, 19 / 31$, respectively) (RR $1.58,95 \% \mathrm{Cl}$ 1.19 to 2.11; Analysis 41.1). We rated the certainty of evidence as moderate, rating down for serious imprecision (only one study).

\section{Adverse effects}

In all intervention groups mild to moderate application-site reactions were reported. See Table 2 for further AE data from this study.

\section{Pulsed dye laser (PDL)}

\section{One session versus two sessions}

One small pilot study (Abd El-Naby 2019) randomised 22 participants with $22 \mathrm{BCCs}(17 \mathrm{nBCCs}$, three $\mathrm{sBCC}$, one pigmented $B C C$ and one cystic $B C C$ located on the face/scalp) to treatment with one or two sessions (two weeks apart) of PDL $(595 \mathrm{~nm}$, fluency 7 $\mathrm{J} / \mathrm{cm}^{2}$, pulse width $20 \mathrm{~ms}$, rate $1 \mathrm{~Hz}$ with $4 \mathrm{~mm}$ margins) with six months follow-up.

\section{Secondary outcomes}

\section{Early treatment failure}

Four weeks after the last treatment all lesions were excised and early treatment failure rates were very high. Two sessions of PDL may slightly reduce the rate of early treatment failures compared to one session of PDL (81.9\%, 9/11 versus $100 \%, 11 / 11$, respectively) (RR $0.83,95 \% \mathrm{Cl} 0.60$ to 1.13 ; Analysis 42.1 ). We rated the certainty of evidence as moderate, rating down for serious imprecision ( small numbers involved in study and wide $95 \% \mathrm{Cl}$ ).

\section{Adverse effects}

The paper reported the technique was tolerable and safe with minimal side effects but no further data were given.

\section{$7 \mathrm{~mm}$ spot size single pulses versus $10 \mathrm{~mm}$ spot size stacked pulses}

One small pilot study (Tran 2012) randomised 17 participants with $20 \mathrm{BCCs}$ ( $13 \mathrm{nBCCs}, 6$ multifocal BCCs, one superficial BCC located on trunk/limbs) into three treatment groups: a control (no treatment) group; PDL (595 nm, fluency $15 \mathrm{~J} / \mathrm{cm}^{2}$, pulse width $3 \mathrm{~ms}$ ) using $7 \mathrm{~mm}$ spot size and single pulses and PDL ( $595 \mathrm{~nm}$, fluency 7.5 $\mathrm{J} / \mathrm{cm}^{2}$, pulse width $3 \mathrm{~ms}$ ) using $10 \mathrm{~mm}$ spot size and double-stacked pulses. All lesions were treated with a $4 \mathrm{~mm}$ margin of clinically normal skin. The rationale for increasing spot size and stacking pulses is to increase the depth of penetration of the laser. Three SCC-in-situ lesions were also treated and we have excluded these from our analyses. We have not analysed the control group data as no placebo/sham treatment was given.

\section{Secondary outcomes}

\section{Early treatment failure}

Six weeks after treatment, all treated lesions were excised. PDL with a $10 \mathrm{~mm}$ spot-size and stacked pulses may reduce early treatment failure rates compared to PDL with a $7 \mathrm{~mm}$ spot-size and single pulses (28.6\%, $2 / 7$ versus $85.7 \%, 6 / 7$, respectively) (RR $0.33,95 \%$ $\mathrm{Cl} 0.10$ to 1.12 ; Analysis 43.1 ). We rated the certainty of evidence as moderate, rating down for serious imprecision (small numbers involved in study and wide $95 \% \mathrm{Cl}$ ).

\section{Adverse effects}

Mild scarring occurred in both treatment arms and by five months post-treatment hypopigmentation was noted. See Table 2 for further AE data from this study.

\section{PDL versus control (sham laser or no treatment)}

One study (Karsai 2015) randomised 39 participants (100 sBCC on trunk/extremities) to PDL (wavelength $595 \mathrm{~nm}$; fluence $8 \mathrm{~J} \mathrm{~cm}^{2}$; pulse duration $0.5 \mathrm{~ms}$; spot size $10 \mathrm{~mm}$ ) or sham treatment with six months follow-up. A total of four treatment sessions at three-week intervals were conducted by a dermatologist experienced in laser therapy. Another small pilot study (Eimpunth 2014) randomised 24 participants (24 sBCC or $\mathrm{nBCC}$ ) to PDL or no treatment. The PDL treatment was one session of double-stacked pulses of PDL using $10 \mathrm{~mm}$ spot size, $3 \mathrm{~ms}$ pulse width and a fluence of $7.5 \mathrm{~J} / \mathrm{cm}^{2}$ with a $6 \mathrm{~mm}$ margin of normal skin around the clinically apparent tumour also treated. Lesions were all subsequently excised and histologically assessed.

\section{Secondary outcomes \\ Pain during and after treatment}

Pain was recorded on VAS (0-10) during treatment. Data presented did not allow for analysis; however, the authors reported that the maximum median score for PDL was $4 / 10$ and for sham was $0 / 10$. See Table 1 for further pain data from this study.

\section{Early treatment failure}

At six months, PDL probably reduces the risk of early treatment failures compared to sham laser $(21.4 \%, 12 / 56$ versus $95 \%, 42 / 44$, respectively) (RR $0.22,95 \% \mathrm{Cl} 0.14$ to 0.37 ; Analysis 44.1 ). We rated the certainty of evidence as moderate, rating down for imprecision (only one study). PDL may improve the risk of early treatment failure compared to no treatment RR $0.36,95 \% \mathrm{Cl} 0.15$ to 0.86 ; Analysis 45.1). We rated the certainty of evidence as moderate, rating down for serious imprecision due to wide $\mathrm{Cl}$ and small sample size. 


\section{Adverse effects}

The main AEs in the PDL group were crusts, hyper- and hypopigmentation. See Table 2 for further AE data from this study.

\section{Intralesional interferon therapy}

Four trials met our inclusion criteria comparing different dosages of interferon and placebo. There were no active comparator studies.

\section{Interferon versus placebo injections}

One study (Cornell 1990) of 165 participants (sBCC and nBCC) compared interferon alpha- $2 \mathrm{~b}$ ( 1.5 million units three times weekly for three weeks) against vehicle with one-year follow-up. Patients were randomised in a 3:1 ratio, interferon:placebo. Another study (Rogozinski 1997) compared recombinant interferon beta (1 million units three times weekly for three weeks) compared to placebo in 35 participants (subtype, size and location of BCC not reported).

\section{Secondary outcomes}

\section{Early treatment failure}

Based on the Cornell 1990 study, when measured at week 20, interferon alpha-2b probably reduces the risk of early treatment failures compared to intralesional placebo $(14.2 \%, 17 / 120$ versus $71.4 \%, 30 / 42$, respectively) (RR $0.20,95 \% \mathrm{Cl} 0.12$ to 0.32 ; Analysis 46.1). We rated the certainty of evidence as moderate, rating down for imprecision (only one study). Based on the Rogozinski 1997 study, interferon beta probably reduces the risk of early treatment failures compared to intralesional placebo $(53 \%, 8 / 15$ versus $100 \%$, 18/18) (RR 0.55, $95 \% \mathrm{Cl} 0.34$ to 0.87 ; Analysis 47.1). We rated the certainty of evidence as moderate, rating down for imprecision (only one study).

\section{Adverse effects}

In the Cornell 1990 study, influenza-like symptoms occurred more commonly in the interferon-treated group than the vehicle group. In the Rogozinski 1997 study, inflammation at the injection site was found in 11 out of 16 participants in the treatment group and four out of 18 receiving placebo. See Table 2 for further AE data from these studies.

\section{Interferon alpha-2a and interferon alpha-2b versus single interferon}

One study (Alpsoy 1996) compared interferon alpha-2a, interferon alpha-2b or both interferon alpha-2a and interferon alpha-2b in 45 participants (sBCC, nBCC and morphoeic BCCs located on the head and trunk) with two years follow-up.

\section{Secondary outcomes}

\section{Early treatment failure}

Interferon alpha-2a and $-2 \mathrm{~b}$ may slightly reduce the risk of early treatment failure compared to interferon alpha-2a (RR $0.80,95 \% \mathrm{Cl}$ 0.27 to 2.41; Analysis 48.1). Interferon alpha- $2 a$ and $-2 b$ may slightly reduce the risk of early treatment failure compared to interferon alpha-2B (RR 0.80, 95\% Cl 0.27 to 2.41; Analysis 49.1). There may be no difference in risk of early treatment failures when comparing interferon alpha-2b with interferon alpha-2a (RR 1.0, 95\% $\mathrm{Cl} 0.36$ to 2.75; Analysis 50.1). We rated the certainty of evidence as low for all these comparisons due to very serious imprecision (very wide $95 \%$ $\mathrm{Cl}$ that indicated important benefit or harm).

\section{Adverse effects}

Pain was experienced at the injection site and all participants had influenza-like symptoms (fever, chills, headaches, fatigue, myalgia) especially within the first two weeks after the initiation of therapy. See Table 1 and Table 2 and for further pain data and AE data from this study.

\section{Sustained release interferon alpha-2b}

One study (Edwards 1990) compared different dosing schedules of sustained release interferon (protamine zinc chelate interferon alpha-2b) in 65 participants with $\mathrm{nBCC}$ and SBCC (location not reported). Participants were randomised to a single dose of 10 million international units (IU) versus the same dose weekly for three weeks.

\section{Secondary outcomes}

\section{Early treatment failure}

The three times weekly injection treatment group probably reduces the risk of early treatment failures compared with the single dose $\operatorname{arm}(20 \%, 6 / 30$ versus $48.5 \%, 16 / 33$, respectively) (RR $0.41,95 \% \mathrm{Cl}$ 0.19 to 0.92 ; Analysis 51.1 ). We rated the certainty of evidence as moderate rating down for serious imprecision (wide $95 \% \mathrm{Cl}$ and only one study).

\section{Adverse effects}

All participants experienced at least one adverse reaction. Side effects were similar for both single and repeated dosage groups, and were those common to interferon. See Table 2 for further $A E$ data from this study.

\section{Topical Sinecatechins}

One study (Kessels 2017) compared treatment with 10\% sinecatechin ointment $\left(\right.$ Veregen $^{\circledR}$ ) with vehicle in 42 participants with SBCC over eight weeks follow-up.

\section{Secondary outcomes}

\section{Early treatment failure}

There is probably little to no difference in the risk of early treatment failures between topical sinecatechin ointment $(95.2 \%, 20 / 21)$ and placebo $(90.5 \%, 19 / 21)$ (RR $1.05,95 \% \mathrm{Cl} 0.89$ to 1.25 ; Analysis 52.1 ). We rated the certainty of evidence as moderate, rating down for serious imprecision (only one study).

\section{Adverse effects}

See Table 2 for further AE data from this study.

\section{Summary of pain data}

Pain was reported on a variety of different scales, for example visual analogue and three point scales. Occasionally, qualitative data was captured; pain could be reported as burning, stinging, throbbing, irritation, lingering, tingling, tenderness or blinking discomfort. Pain was rarely reported distal to the treatment site for example pharyngolaryngeal pain after ingenol mebutate gel usage (Siller 2010). Depending on the study, patients could be asked to report any pain during or after treatment; this could be as an isolated assessment or could be done at intervals. Burning was the most common type of pain reported with PDT (Ezughah 2008; Arits 2013; Foley 2009a and Foley 2009b). Some authors reported supplying pre-treatment analgesia before PDT (Choi 2016, Haak 2015) or SE 
(Rhodes 2004). Others reported that physical measures to induce analgesia during PDT were permitted (Morton 2018; Szeimies 2008; Wang 2001). Post-treatment analgesia was occasionally permitted after SE (Bath-Hextall 2014), imiquimod, radiotherapy (GarciaMartin 2011) and sinecatechin applications (Kessels 2017). Pain and tenderness during treatment with imiquimod was expected, and participants were frequently allowed a rest period if needed in order to complete the treatment course (Bath-Hextall 2014; Ezughah 2008; Geisse 2002; Geisse 2004; Shumack 2002a; Shumack 2002b; Sterry 2002a and Sterry 2002b) with occasional participants unable to complete the treatment due to pain despite this (Ezughah 2008; Shumack 2002b). In total five patients discontinued treatment due to pain; four after imiquimod application (Ezughah 2008; Shumack 2002a; Shumack 2002b) and one after MAL-PDT (Rhodes 2004). For further details see Table 1 and Table 2.

\section{Summary of adverse effects data}

Patient-reported adverse effects did not always align with those reported by the observer (Eigentler 2007). The most commonly reported adverse effects were due to application site reactions seen in the non-surgical interventions. These manifested as localised inflammation resulting in: itching, weeping, crusting, scabbing, flaking, erythema, blistering, vesicles, eschar, erosions, burning, pain, purpura or swelling. Localised inflammation was reported in 13 of the 14 papers on imiquimod usage (Arits 2013; Bath-Hextall 2014; Eigentler 2007; Ezughah 2008; Garcia-Martin 2011; Geisse 2002; Geisse 2004; Marks 2001; Schulze 2005; Shumack 2002a; Shumack 2002b; Sterry 2002a;Sterry 2002b).

Other treatment modalities resulting in localised inflammation include AFXL-PDT (Haak 2015), intralesional 5-FU (Romagosa 2000), PDL (Eimpunth 2014; Karsai 2015; Tran 2012), radiotherapy (GarciaMartin 2011), sinecatechins (Kessels 2017), laser/broadband light ALA-PDT (Soler 2000), tazarotene and valproic acid (2005-001474-27) and placebo/vehicle (Foley 2009a; Foley 2009b; Geisse 2002; Kessels 2017; Siller 2010).

Local wound infection or suppuration were reported after surgical treatments such as SE (Kuijpers 2007; Rhodes 2004; Smeets 2004; Szeimies 2008), cryosurgery (Basset-Seguin 2008; Kuijpers 2007; Wang 2001), cryosurgery and curettage (Kuijpers 2007) as well as non-surgical interventions: imiquimod (Arits 2013), 5-FU (Arits 2013), ALA-PDT (Mosterd 2008), but not after MAL-PDT (Rhodes 2004; Szeimies 2008).

Dyspigmentation was seen after treatment with PDL (Abd El-Naby 2019; Eimpunth 2014; Karsai 2015, Tran 2012), imiquimod (Eigentler 2007; Schulze 2005), AFXL-PDT, MAL-PDT (Haak 2015) and in 83\% of treated with intralesional 5-FU (Miller 1997).

Reports of radiodystrophy and necrosis decreased over time after radiotherapy treatment (Avril 1997). Necrosis (of grafts) was also seen after SE (Smeets 2004). Scarring was seen after the use of PDL (Eimpunth 2014), AFXL-PDT, MAL-PDT (Haak 2015) and ingenol mebutate gel (Siller 2010). "Adverse" scarring was noted after SE (Avril 1997).

Headache was statistically more prevalent after treatment with certain imiquimod regimens (five times per week) than in placebo groups (Geisse 2004). Imiquimod also induced influenza-like symptoms (Bath-Hextall 2014; Schulze 2005; Shumack 2002a; Shumack 2002b; Sterry 2002a) and deranged haematology results
(Geisse 2004). Biochemical abnormalities were seen with ingenol mebutate gel usage (Siller 2010).

Withdrawal from study, discontinuation of therapy, interrupted therapy regimens or dose reduction due to treatment-related adverse effects occurred with imiquimod (Bath-Hextall 2014; Ezughah 2008; Geisse 2002, Geisse 2004; Marks 2001; Schulze 2005; Shumack 2002a; Shumack 2002b; Sterry 2002a; Sterry 2002b), diclofenac with or without calcipotriol (Brinkhuizen 2016), MALPDT (Foley 2009a; Foley 2009b; Morton 2018; Rhodes 2004), BF 200 ALA-PDT (Morton 2018), solasodine glycosides (Punjabi 2008) and ingenol mebutate gel with or without occlusion (Siller 2010; Spelman 2014).

Serious adverse effects requiring hospitalisation due to treatment were not specifically reported in any treatment group but the influenza-like symptoms and wound infections that were seen with small numbers treated with imiquimod and 5-FU in the Arits 2013 study were classed as suspected unexpected serious adverse reactions. More details regarding reaction severity, duration of adverse events and changes over treatment course can be found in Table 2.

\section{DISCUSSION}

\section{Summary of main results}

Basal cell carcinoma (BCC) is the most common skin cancer with several different interventions available for high- and lowrisk histological subtypes. We aimed to assess the effectiveness of these interventions for primary BCC in immunocompetent individuals and found 52 randomised controlled trials (RCTs) (26 new to this review update) that randomised a total of 6690 participants. We synthesised the data into a total of 52 comparisons. All studies included a majority of BCCs of low-risk histological subtypes (nodular (nBCC), superficial (sBCC)), with only four studies including a minority of high-risk histological subtypes. No studies exclusively studied high-risk histological subtypes and we were unable to retrieve the raw data specific to the high-risk BCCs for full outcome assessment. Consequently, there was not enough information to draw any conclusions about the effects of interventions on histologically high-risk BCC.

Of the included studies, pooling of the data was feasible for only a very limited number of the reported outcomes, and the largest number of studies we were able to pool was four. Many comparisons were evaluated by only one study, preventing meta-analysis. We considered surgical excision (SE) versus Mohs micrographic surgery (MMS) as the main comparison in this review. However, only one study (Smeets 2004), which provided only lowcertainty evidence, assessed MMS, even though this is often used as the gold-standard for high-risk facial BCC.

Broadly, non-surgical interventions were more commonly assessed than surgical interventions: more than double the number of trials assessed non-surgical compared to surgical interventions. The most assessed interventions were photodynamic therapy (PDT) (24 studies); topical imiquimod (14 studies); and surgical excision, which is often regarded as the gold-standard treatment for BCC (10 studies). Eleven studies assessed MAL-PDT; 6, ALAPDT; 2, ablative fractional laser MAL-PDT, and single studies assessed HAL-PDT, laser and broadband ALA-PDT. Between two or four studies assessed radiotherapy, cryosurgery, fluorouracil, 
intralesional interferon, pulsed dye laser, and ingenol mebutate. No more than one study assessed curettage and cautery, electrochemotherapy, solasodine glycosides, valproic acid and tazarotene gel, sinecatechins, calcitriol and diclofenac.

Safety was the most commonly-evaluated outcome, with $81 \%$ of the comparisons assessing adverse effects. Seventy-five per cent of comparisons assessed early treatment failure, $21 \%$ reported 3year recurrence, $17 \%$ reported 5 -year recurrence, $37 \%$ reported on cosmetic outcomes ( $27 \%$ had data for analysis), and $46 \%$ reported on pain ( $19 \%$ had data for analysis).

\section{Surgical excision (SE)}

Surgical interventions have the lowest rates of recurrence at threeand five-year follow-up.

We found that for high-risk facial BCC, there may be slightly fewer recurrences with MMS compared to SE at three years and five years (low-certainty evidence). However, the 95\% confidence interval (Cl) also includes the possibility of an increased risk of recurrence and no difference between treatments. There may be little to no difference regarding improvement of participant- and observerrated cosmetic outcomes (low-certainty evidence) measured at 18 months post-operatively; however, no raw data were available for this outcome.

Smeets 2004 has been criticised for performing MMS with a standardised $3 \mathrm{~mm}$ margin as normally smaller margins are used and this larger margin may have diminished the tissue-preserving benefit of MMS, potentially worsening the cosmetic outcome (Muller 2009). The study included high-risk facial BCCs, defined as high-risk histological subtypes (infiltrative, micronodular, morphoeic, BCC with squamous differentiation) or BCCs located in the 'H-zone' of the face, or both.

The study followed up participants for over 10 years, and although our primary outcome was recurrence at three years and five years, it is important to note that during the five- to 10-year followup period there were an additional 14 recurrences (four in the MMS group and 10 in the SE group), which means that only $44 \%$ of BCC recurrences occurred during five years of follow-up. This is important as clinicians and patients should be aware that recurrences are possible up to 10 years following treatment.

For further details see Summary of findings 1 .

\section{Imiquimod}

Evidence for low-risk $\mathrm{sBCC}$ and $\mathrm{nBCC}$ found that imiquimod probably results in more recurrences than SE at three years (10fold increased risk, moderate-certainty evidence) and at five years (eight-fold increased risk, moderate-certainty evidence). This was a non-inferiority study and imiquimod was found to be inferior to SE as it failed to meet the study's pre-defined inferiority margin of 0.87 . When measured at three years, there may be little to no difference between imiquimod and SE in the number of participantrated good/excellent cosmetic outcomes (low-certainty evidence), but when this outcome is observer-rated, imiquimod may increase good/excellent cosmetic outcomes (low-certainty evidence). This discrepancy between participant- and observer-rated cosmetic outcomes may be important and should be highlighted to patients.
Although inferior to SE in terms of reducing recurrence rates, imiquimod's performance is still clinically acceptable to patients and clinicians and it is probably the best non-surgical treatment option for low-risk BCC. Another important benefit of imiquimod resides in the fact that it can be administered by patients at home, offering the opportunity to manage low-risk BCCs in the community. With the trend for patients presenting younger and with increasing numbers of BCCs, some now advocate the possibility of general practitioners diagnosing and managing low-risk BCCs with imiquimod cream and reserving SE for the approximate $1 / 5 \mathrm{BCC}$ s that relapse, with the majority found to relapse within the first year following treatment (Kelleners-Smeets 2017).

Side effects of imiquimod cream comprise application site reactions including pain, weeping, itching, redness and swelling and were reported more often in the imiquimod group than in the SE group. The licensed treatment regimen is five days per week dosing for six weeks (sBCC) or 12 weeks (nBCC). Bath-Hextall 2014 used daily dosing (the discrepancy may have occurred as imiquimod was licensed for BCC after the study had commenced) and therefore more adverse effects may have been recorded during the study than would be expected in clinical practice. Recurrences recorded at five years compared with three years were small, with one additional recurrence for a SBCC treated with imiquimod and one after SE.

Out of 14 studies assessing imiquimod, 11 were funded by the science-based technology company $3 \mathrm{M}$.

For further details see Summary of findings 2.

\section{Radiotherapy}

In comparison against SE with the option of frozen section margin control for facial BCCs (including high- and low-risk subtypes), radiotherapy may result in increased recurrences at three years (low-certainty evidence) and at four years (low-certainty evidence). When measured at four years, radiotherapy probably results in a lower number of good cosmetic outcomes (irrelevant of whether the outcome was participant- or observer-rated) compared to SE (moderate-certainty evidence), as dyspigmentation and telangiectasia can occur in people treated with radiotherapy.

Different radiation techniques exist (such as conventional X-ray therapy, interstitial brachytherapy and superficial contact therapy) with the choice of technique dependent on factors such as size and location of the lesion and performance status of the patient. In practice, radiotherapy is usually reserved for cases where lesions are not amenable to surgery. When deciding whether to choose radiotherapy or SE, patients need to consider that radiotherapy will probably involve numerous visits to the radiotherapy department up to five times a week, usually for several weeks. It should be noted that the RCTs for radiotherapy were all conducted decades ago. Since these studies were carried out, radiotherapy technology and protocols have improved and therefore these results may not be representative of modern radiotherapy outcomes. New studies comparing modern radiotherapy against other interventions are needed.

For further details see Summary of findings 3. 


\section{Photodynamic therapy (PDT)}

PDT was studied in 16 RCTs against various surgical and nonsurgical interventions. Out of 16 PDT studies, seven were industryfunded (five by PhotoCure/Galderma).

Based on participants with low-risk nBCC on the head and neck region, MAL-PDT may result in more recurrences than SE at three years (low-certainty evidence); there were no usable data for assessment at five years. MAL-PDT probably has slightly more participant- and observer-rated good/excellent cosmetic outcomes at one year compared to SE, based on participants with low-risk $\mathrm{nBCC}$ and SBCC (moderate-certainty evidence). For further details see Summary of findings 4.

Based on participants who each had a single low-risk SBCC, imiquimod probably results in fewer recurrences at three years and five years compared to MAL-PDT (moderate-certainty evidence), and there is probably little to no difference in observer-rated good/excellent cosmetic outcomes measured at one year between MAL-PDT and imiquimod (moderate-certainty evidence). This comparison did not measure participant-rated cosmetic outcomes. For further details see Summary of findings 5.

There is wide variation in PDT protocols with differences in photosensitising agent, use of prior debulking curettage, timing, source and amount of light irradiation and number of PDT cycles. MAL-PDT predominates in Europe and Australasia; physicians in the USA mainly use ALA-PDT. The standard European protocol defines one cycle as two PDT sessions separated by a one-week interval. MAL-PDT is significantly more expensive than ALA-PDT. 5-ALA, however is disadvantaged by the need to prepare the cream fresh prior to use whereas MAL is available in a standardised preparation which makes it easier to use. The newer, stable nanoemulsion ALA formulation (BF-200 ALA gel) is now licensed in the EU. HAL has been used for years in bladder cancer detection and its use in BCC treatment has now been investigated. Further studies are required to inform which protocol is most effective.

Arits 2013 identified that MAL-PDT probably leads to fewer recurrences compared to imiquimod for SBCC treatment, in one subgroup of patients only: older patients with SBCC on the lower extremities.

\section{Adverse effects}

Adverse effects with surgical interventions included wound infections, necrosis of grafts and post-operative bleeding. Local adverse effects such as itching, weeping, pain and redness occurred frequently with non-surgical interventions. Treatment-related adverse effects resulting in study modification or withdrawal occurred with imiquimod and MAL-PDT.

For further details see Table 4, Table 5, Table 8, Table 3, Table 7, and Table 6.

\section{Overall completeness and applicability of evidence}

This systematic review has included the full spectrum of interventions for primary BCC by including 52 RCTs $(52$ comparisons) of varying methodological quality. The evidence we have identified addressed our primary and secondary outcomes to varying degrees. Only $21 \%$ of the comparisons we included presented at least three-year recurrence data, and 17\% of comparisons reported 5-year recurrence data. Given that the majority of recurrences are expected to occur by three years, this means that almost $80 \%$ of studies were not able to address our primary recurrence rate outcomes due to inadequate followup time. This threatens the applicability and external validity of the evidence presented. Histologically-confirmed early treatment failure data were available for all but 13 comparisons. This outcome benefits from not requiring a long follow-up period and was frequently used as a primary outcome in small, pilot phase $1 / 2$ studies but is generally of limited use when informing decisions in clinical practice.

The finding in Smeets 2004 that $56 \%$ of recurrences of high-risk facial BCC occur between five and 10 years following surgery suggests that longer follow-up times are needed for studies on BCC and may represent a flaw in our review methodology. However, a large retrospective review of long-term BCC recurrence rates identified that only $18 \%$ of recurrences occurred between five and 10 years with $67 \%$ found to occur within the first three years (Rowe 1989). This study informed our original review's three-year time point. Further retrospective studies have reported that $18 \%$ to $20 \%$ of recurrences occur beyond five years (Griffiths 2005; Hruza 1994; Randle 1996).

Nineteen comparisons had cosmetic outcome data, of which 14 had data for analysis. There was heterogenous reporting of cosmetic outcomes, using different time points, scales and rater (e.g. participant, observer or both). Observers were generally medically trained (e.g. dermatologist, surgeon), but some studies used nonmedical observers. Histologically-confirmed early treatment failure was available for all but 13 comparisons. Although 24 studies reported on pain, we were only able to perform analyses on pain data for 10 comparisons; the severity and analgesia required were not routinely reported, and the modes of assessment ranged from patient diaries to rating scales or visual analogue scores. Most studies presented this in their adverse effects data, and due to the heterogeneity, we have collated adverse effects and pain data into additional tables (Table 1 and Table 2).

The diversity of the outcome data generated by these trials impacted our ability to make direct comparisons between the studies; therefore, these data are occasionally not reported. The discrepancy in reported outcomes and the inconsistency in reported time points underscores the importance of the work being done to create core outcome sets based on the needs of the stakeholders for clinical trials in BCC (Schlessinger 2017).

Thirteen comparisons involved a placebo/vehicle/sham/no treatment arm, and imiquimod was over-represented in this group, with five studies comparing imiquimod against vehicle, involving 1145 participants. This allowed one of the few metaanalyses included in this review. It should also be noted that all of these imiquimod studies were industry-funded. BCC is a disease associated with significant local tissue destruction and therefore the harm of non-treatment is not insignificant. It is for this reason that most placebo-controlled studies had short follow-up periods and usually excised the treatment area at the end of the study. Twenty-six comparisons were head-to-head, and such studies are more applicable to clinical practice as they answer more pragmatic and clinically relevant questions.

We do not have sufficient evidence to determine the effects of treatment for histologically high-risk BCC, because only four of our included studies assessed this subtype and no studies 
exclusively included high-risk histological subtypes. Only three studies exclusively assessed BCCs located at high-risk sites (H-zone of the face), and so there was insufficient evidence to determine the effects of treatment for these BCCs.

The vast majority of participants were elderly with a median age of 64.9 years old. Although participants as young as 20 were included, separate data on younger participants were not available and therefore we do not have sufficient evidence to determine if outcomes are different in younger participants.

Only one study provided evidence for our main comparison of surgical excision versus MMS. Non-surgical interventions were most widely evaluated by our included studies, which correlates with the inclusion of low-risk histological BCC subtypes by all of the studies.

Three types of treatment were disproportionately represented compared to the rest of the treatments considered: 48 trials assessed PDT, topical imiquimod, and surgical excision, whereas almost half of that number (26 studies), in total, assessed the following: radiotherapy, cryosurgery, fluorouracil, intralesional interferon, pulsed dye laser, ingenol mebutate, curettage and cautery, electrochemotherapy, solasodine glycosides, valproic acid and tazarotene gel, sinecatechins, and diclofenac.

Most of the evidence for the outcomes presented for each of the interventions has come from relatively small, single studies, which meant that meta-analysis was largely not possible (the exceptions being PDT versus SE, imiquimod versus vehicle and AFXL-PDT versus PDT). The majority of these single studies were multi-centre, but many were limited by small sample sizes, and consequently, many of the outcomes reported in this review have wide confidence intervals. This means there is a large amount of imprecision in the results, and therefore, several of our results have low-certainty evidence which threatens their external validity and reproducibility.

Despite these limitations, our study is the most up-to-date and extensive systematic review on interventions for BCC. It can be used globally to inform guidelines and to aid clinicians and patients in weighing up the risks and benefits of the various treatment options available for primary BCC.

\section{Certainty of the evidence}

The GRADE approach was used to rate the certainty of all the evidence in our review (as outlined in the Methods section). There was a wide variation in the certainty with most outcomes being rated as moderate to low certainty. A few outcomes were rated as very low-certainty, although these were assessed by comparisons not considered key. The main reasons for rating down the certainty of evidence related to serious risk of bias in the studies (for instance, high risk of selection bias) and serious imprecision with small sample sizes and wide confidence intervals. Where confidence intervals included important benefit and serious harm (i.e. > 1.25 and $<0.75$ ), we rated down two levels for very serious imprecision. A few comparisons had such wide $95 \% \mathrm{Cls}$ (including 1- and more than 100-fold difference) that we rated them down three levels for imprecision.

Only 19 studies were assessed as low risk of bias for blinding of the outcome assessor. For the studies that were unblinded we did not rate down the certainty of evidence for recurrence outcomes as it was unclear if being unblinded would bias the assessments. We did rate down certainty of evidence for high risk of bias when there was unblinded assessment of cosmetic outcomes.

Only one study was identified as having a high risk of selection bias, but 22 studies were rated as unclear risk of bias due to the method of random sequence generation not being clearly stated by the study authors. Twenty-eight studies had unclear risk of bias due to the method of allocation concealment not being stated. All these studies stated that they randomised their participants, and therefore, we have had to assume that they did this correctly and we have not rated down the certainty of evidence for an unclear risk of selection bias assessment. Only six studies were deemed to be at low risk of selective outcome reporting bias, and only 11 studies prospectively registered. This number is very low, and selective outcome reporting may have occurred in studies rated as 'unclear' risk of bias, but we decided not to rate the certainty of evidence down for this. This stresses the importance of having pre-specified outcomes and prospectively registering studies.

The majority of included studies used participants as the unit of analysis; however, $27 \%$ of studies reported results based on lesions. Therefore, there is the potential of under-estimating the variation in the intervention effect from using results which are based on the same people contributing with multiple lesions. However, the majority of these studies included small numbers of people with multiple lesions; therefore, we anticipate that the likely impact on our results is minimal. Additionally, all of the latter studies aimed to treat the lesions individually, rather than using a systemic treatment.

\section{Potential biases in the review process}

We attempted to conduct a comprehensive search for studies. Review authors independently assessed eligibility of studies to minimise bias in the study selection process. As we were working from an old protocol, we did decide to make some changes to our methods that have been clearly described in the Differences between protocol and review section. Changes to our protocol were made at the start of the review process and were approved by Cochrane Skin editorial team. Bias may have been introduced by the time points chosen for some of our outcomes. For instance, when faced with cosmetic outcome data with a range of time points, we had to make a decision on which time point to include for the different comparisons. We attempted to minimise this bias by asking HW to decide on what should be the best time point to include. HW decided we should select a time point at least one year following treatment. The rationale being that scars often take around one year to heal and for the colour to settle. He was not aware of the included studies or any of the results when making this decision, so the risk of bias should be minimal, but ideally this should have been pre-specified in the protocol. For the SE versus radiotherapy study cosmetic outcome, we reported the time point with the biggest difference between interventions which was four years after treatment which may have introduced selective outcome reporting bias. The reason we reported this time point is because it is well documented that radiotherapy has deteriorating cosmetic outcomes over years and this would not be appreciated if we reported the one-year time point. Selective outcome reporting bias may also have been introduced by us selecting the maximum pain score recorded during treatment for interventions. We felt this was necessary as pain experienced during treatments is highly variable and depends on the treatment modality. For example, comparing pain on day one for a surgical treatment against a non- 
surgical treatment like imiquimod would be meaningless as the surgical intervention will have produced a painful wound whereas imiquimod will likely not have had any effect on day one.

\section{Agreements and disagreements with other studies or reviews}

There have been a few systematic reviews assessing interventions for BCC published since our last update. Most recently, Drucker 2018 published a comprehensive systematic review and network meta-analysis (NMA) on treatments for primary BCC. This study only included English-language publications, and their search yielded fewer randomised controlled trials (RCTs) whilst also including non-randomised studies. This study also did not specify a time point for recurrence before performing the NMA. Despite these differences, our conclusion that surgical interventions have the lowest recurrence rates for BCC is consistent with theirs. Collier 2018 published a systematic review of PDT for BCC. This study included 15 RCTs and had similar outcome measures as our study, except recurrence at one year and five years were their chosen time points. Our review largely agrees with the results from this review. A systematic review and meta-analysis on PDT for BCC by Wang 2015 identified eight RCTs, of which seven are included in our review. This review pooled the results of all these studies regardless of PDT method including photosensitising agent (e.g. MAL versus ALA). We decided against pooling all types of PDT as the pharmacology for the photosensitising compounds is different and the evidence for their similar efficacy is of low quality. We disagree with the Wang study where they report that PDT is as effective as pharmacologic treatment (imiquimod and 5-FU creams). The Wang study combined both imiquimod and 5-FU in their analyses, and by doing so, they masked imiquimod's probable superior efficacy to PDT in terms of reducing recurrences.

\section{Risk of transformation with non-invasive treatments?}

We thought it was important to address the fairly recent literature that has suggested that topical therapies and PDT may cause nonaggressive sBCCs to "transform" to more aggressive subtypes (e.g. nodular, infiltrative or morphoeaform subtypes). This is an area of contention and others have argued that apparent transformation is more likely the result of sampling error from the diagnostic biopsies. The Arits 2013 study (MAL-PDT versus imiquimod versus 5-FU cream) data were used for a separate observational study to address the question of whether non-invasive therapies cause sBCCs to transform to higher risk subtypes (van Delft 2019). The study found that of 166 treatment failures (38.6\%) were a nonsuperficial subtype and the proportion with a more aggressive subtype than the primary tumour were $51.3 \%$ (38.74) for early and $28.3 \%(26 / 92)$ for later treatment failures. The authors conclude that more aggressive treatment failure recurrences after noninvasive therapy for SBCC occur most often within the first three months following treatment, probably indicating sampling error and consequent under-diagnosis of more aggressive components in the primary tumour rather than transformation.

\section{AUTHORS' CONCLUSIONS}

\section{Implications for practice}

The overall quality of research on interventions for basal cell carcinoma (BCC) has improved since our 2007 review with the publication of several randomised controlled trials (RCTs) with long-term follow-up, but many of the included studies have provided low- or moderate-certainty evidence that should be interpreted with caution.

The majority of studies have been performed on low-risk histological BCCs, located on low-risk sites, the results of which are probably not applicable to high-risk tumours. Only four studies have looked at high-risk histological subtypes, and three studies looked at BCCs at high-risk facial sites. More studies or subgroup analyses are required for morphoeic tumours.

Surgery remains the most effective treatment modality for BCC in terms of reducing recurrences, and there may be a slightly reduced recurrence rate with Mohs micrographic surgery (MMS) compared to surgical excision (SE); however, the $95 \% \mathrm{Cl}$ also includes the possibility of both increased risk and no difference between treatments (low-certainty evidence). With regard to improvement of participant- and observer-rated cosmetic outcomes, there may be little to no difference between MMS and SE (low-certainty evidence); however, no raw data were available for this outcome. Radiotherapy is effective but probably worse than surgery (under frozen section margin control) in terms of the number of good cosmetic outcomes (moderate-certainty evidence) and is therefore best reserved for tumours not amenable to surgery. Radiotherapy may also lead to increased recurrence compared to SE (lowcertainty evidence).

Non-surgical treatments are less effective, but the evidence suggests that recurrence rates are acceptable and they are important options to offer patients. Imiquimod probably results in more recurrences than SE (moderate-certainty evidence) and there is probably little to no difference between groups in the number of participant-rated good/excellent cosmetic outcomes (low-certainty evidence). However, imiquimod may increase the number of observer-rated good/excellent cosmetic outcomes compared to SE (low-certainty evidence). Overall, imiquimod has the best evidence to support its efficacy out of the non-surgical treatments.

Moderate-certainty evidence indicates that imiquimod probably leads to fewer recurrences than MAL-PDT and there is probably little to no difference between these treatments in terms of observer-rated good/excellent cosmetic outcomes (participantrated cosmetic outcomes were not measured in this comparison). MAL-PDT may result in more recurrences at three years than SE (low-certainty evidence; no useable data for measurement at five years), but probably increases the number of good/excellent cosmetic results (moderate-certainty evidence).

Adverse effects with surgical interventions include wound infections, graft necrosis and post-operative bleeding. Local adverse effects such as itching, weeping, pain and redness occur frequently with non-surgical interventions. Treatment-related adverse effects resulting in study modification or withdrawal occurred with imiquimod and MAL-PDT.

The six studies in Studies awaiting classification may alter the conclusions of the review once assessed.

\section{Implications for research}

Currently, there is only low-certainty evidence informing us on the efficacy of MMS against SE, with only one study assessing MMS against SE that met our inclusion criteria. Given this is the main comparison of our review, further studies assessing these treatments against each other are needed. Future studies 
exclusively assessing interventions for high-risk BCC (high-risk histological subtypes and high-risk locations) are needed. As a minimum, future studies should present data on high-risk tumours in subgroup analyses.

Cost:benefit ratios have an important impact on clinical practice and guidelines and so future systematic reviews on interventions for BCC should also include cost-analyses as an outcome measure. This will allow us to answer important questions such as whether the higher operative costs and procedure times of MMS offset the potential higher number of recurrences seen with SE over time. It will also be important for imiquimod and 5-FU cream as there is a big cost difference between these treatments, which may offset the differences in efficacy so defining this further will be important in future research to impact clinical practice.

Given that the radiotherapy studies included were all conducted over 20 years ago, and based on the fact that radiotherapy techniques and protocols have changed a lot since then, up-todate studies assessing the efficacy of radiotherapy using current methods are needed, as these older studies may not be reflective of modern radiotherapy outcomes.

One of the key problems with RCTs on interventions for BCC is the lack of an agreed set of core outcome measures and also the lack of longer-term outcomes for many of the studies which are now standard for most other cancer studies (for example, clinical recurrence at five years). An international group, in collaboration with the Core Outcome Measures in Effectiveness Trials (COMET) initiative and Cochrane Skin Group- Core Outcome Set Initiative (CSG-COUSIN) have begun the task of developing broad consensus for a core outcome set for BCC intervention trials, which will be essential to improve the evidence we have for BCC in the future (Schlessinger 2017). For example, core outcome sets would determine whose opinion should be sought for assessing cosmetic outcomes (dermatologist, participant, or both), and when or how best to report it. BCC research should benefit greatly from this initiative, but future studies must also ensure that all outcomes are pre-specified and registered prospectively to mitigate against the risk of selective outcome reporting bias.

Only a fifth of included RCTs followed participants up for at least three years and given that the majority of BCCs are likely to recur during the first three years after treatment, future studies should have follow-up periods of at least three years. Further research is needed into what the optimal length of follow-up should be as there is conflicting evidence documenting recurrences beyond five years. If at least $20 \%$ of recurrences occur beyond five years, then studies should follow up participants for up to 10 years. This will also impact the time points used for our primary outcome measures in future reviews.

Moreover, inadequate reporting of study methodology leads to biased estimates of treatment effects (Schulz 1995). Future studies on interventions for BCC must be well-designed RCTs and should include a power calculation to ensure that sufficient numbers of participants are recruited to avoid problems with imprecision. Studies must also be rigorously reported and conform to the Consolidated Standards of Reporting Trials (CONSORT) statement (Schulz 2010). This will ensure a fair interpretation of the results and allow accurate appraisal and judgements to be made about the risk of bias and the overall certainty of evidence.

Given that curettage and cautery is commonly used in the treatment of low-risk BCC, more evidence is needed to assess its efficacy as currently we only have one small study with only two years follow-up that compared curettage and cautery with SE. Further RCTs with long-term follow-up are needed to assess its efficacy against other interventions including non-surgical interventions. Further RCTs with long-term follow-up are needed for solasodine glycosides, ingenol mebutate, topical valproic acid, tazarotene, diclofenac, calcitriol and sinecatechin. These nonsurgical interventions should be compared against other nonsurgical interventions with established efficacy in treating BCC, such as imiquimod.

\section{ACKNOWLEDGEMENTS}

Many thanks to the Cochrane Skin team for all their support, advice and patience.

We also want to thank Jack Tweed who was the consumer and checked the plain language summary for readability and clarity.

The Cochrane Skin editorial base wishes to thank Urbà González, Cochrane Dermatology Editor for this review; Laurence Le Cleach, Methods Editor; Gil Weintraub and Victoria RV Cox, the clinical referees; Kathie Godfrey, consumer referee; Heather Maxwell who copy-edited the review; and Nicole Pitcher who wrote the plain language summary. 


\section{R E F E R E N C E S}

\section{References to studies included in this review}

2005-001474-27 \{unpublished data only\}

2005-001474-27. A randomised, double-blinded parallel group study to compare efficacy and tolerability of topically applied Baceca and Tazarotene against placebo and Tazarotene in patients with basal cell carcinoma. www.clinicaltrialsregister.eu/ctr-search/trial/2005-001474-27/ results (first accessed 12 August 2016).

\section{Abbade 2015 \{published data only\}}

Abbade L, Gige T, Amaral V, Aquino S, Miot H. Higher recurrence rates of head and neck nodular basal cell carcinoma treated with topical photodynamic therapy (MAL-PDT) compared to surgical excision: a randomized controlled study. Journal of the American Academy of Dermatology 2015;72(5 Suppl 1):AB185. [CENTRAL: CN-01076055]

\section{Abd El-Naby 2019 \{published data only\}}

Abd El-Naby NM, El-Far NN, Al-Shenawy H A, Elshwadfy SE, Koura AA. Pulsed dye laser in the treatment of basal cell carcinoma: a single session versus two sessions-a randomized controlled trial. Indian Journal of Dermatology, Venereology, and Leprology 2019;85(5):475.

\section{Alpsoy 1996 \{published data only\}}

Alpsoy E, Yikmaz E, Basaran E, Yazar S. Comparison of the effects of intralesional interferon alfa- $1 a, 2 b$ and the combination of $2 a$ and $2 b$ in the treatment of basal cell carcinoma. Journal of Dermatology 1996;23(6):394-6. [CENTRAL: $\mathrm{CN}-00127615]$

\section{Arits 2013 \{published data only\}}

* Arits AH, Mosterd K, Essers BA, Spoorenberg E, Sommer A, De Rooij MJ, et al. Photodynamic therapy versus topical imiquimod versus topical fluorouracil for treatment of superficial basalcell carcinoma: a single blind, non-inferiority, randomised controlled trial. Lancet Oncology 2013;14(7):647-54. [CENTRAL: $\mathrm{CN}-00872324]$

Jansen $\mathrm{MH}$, Koekelkoren FH, Nelemans PJ, Arits AH, Roozeboom MH, Kelleners-Smeets NW, et al. Comparison of long-term cosmetic outcomes for different treatments of superficial basal cell carcinoma. Journal of the American Academy of Dermatology 2018;79(5):961-4. [CENTRAL: CN-01703594]

Jansen MH, Mosterd K, Arits AH, Roozeboom MH, Sommer A, Essers BA, et al. Five-year results of a randomized controlled trial comparing effectiveness of photodynamic therapy, topical Imiquimod, and topical 5-fluorouracil in patients with superficial basal cell carcinoma. Journal of Investigative Dermatology 2018;138(3):527-33. [CENTRAL: CN-01457400]

Roozeboom MH, Arits AH, Mosterd K, Sommer A, Essers BA, de Rooij MJ, et al. Three-year follow-up results of photodynamic therapy vs. imiquimod vs. fluorouracil for treatment of superficial basal cell carcinoma: a single-blind, noninferiority, randomized controlled trial. Journal of Investigative
Dermatology 2016;136(8):1568-74. [CENTRAL: CN-01197852] [DOI: 10.1016/j.jid.2016.03.043]

\section{Avril 1997 \{published data only\}}

* Avril MF, Auperin A, Margulis A, Gerbaulet A, Duvillard P, Benhamou $E$, et al. Basal cell carcinoma of the face: surgery or radiotherapy? Results of a randomized study. British Journal of Cancer 1997;76(1):100-6. [CENTRAL: CN-00141470]

Petit JY, Avril MF, Margulis A, Chassagne D, Gerbaulet A, Duvillard $\mathrm{P}$, et al. Evaluation of cosmetic results of a randomised trial comparing surgery and radiotherapy in the treatment of basal cell carcinoma of the face. Plastic and Reconstructive Surgery 2000;105(7):2544-51. [CENTRAL: CN-00278207]

\section{Basset-Seguin 2008 \{published data only\}}

Basset-Seguin N, Ibbotson S, Emtestam L, Tarstedt M, Morton C, Maroti $\mathrm{M}$, et al. Methyl aminolaevulinate photodynamic therapy vs. cryotherapy in primary superficial basal cell carcinoma: results of a 36-month follow-up. British Journal of Dermatology 2005;153(1):29.

* Basset-Seguin N, Ibbotson S, Emtestam L, Tarstedt M, Morton C, Maroti M, et al. Topical methyl aminolevulinate photodynamic therapy versus cryotherapy for superficial basal cell carcinoma: a 5 year randomized trial. European Journal of Dermatolology 2008;18(5):547-53. [CENTRAL: CN-00668120]

Bath-Hextall 2014 \{published data only\}

* Bath-Hextall F, Ozolins M, Armstrong SJ, Colver GB, Perkins W, Miller PS, et al. Surgical excision versus imiquimod $5 \%$ cream for nodular and superficial basal-cell carcinoma (SINS): a multicentre, non-inferiority, randomised controlled trial. Lancet Oncology 2014;15(1):96-105. [CENTRAL: CN-00959373]

Williams HC, Bath-Hextall F, Ozolins M, Armstrong SJ, Colver GB, Perkins W, et al. Surgery versus $5 \%$ imiquimod for nodular and superficial basal cell carcinoma: 5-year results of the SINS randomized controlled trial. Journal of Investigative Dermatology 2017;137(3):614-9. [CENTRAL: CN-01340067] [EMBASE: 614596349]

\section{Beutner 1999 \{published data only\}}

Beutner KR, Geisse JK, Helman D, Fox TL, Ginkel A, Owens MA. Therapeutic response of basal cell carcinoma to the immune response modifier imiquimod $5 \%$ cream. Journal of the American Academy of Dermatology 1999;41(6):1002-7. [CENTRAL: CN-00264417]

Brinkhuizen 2016 \{published data only\}10.1016/ j.jaad.2016.01.050

Brinkhuizen T, Frencken KJ, Nelemans PJ, Hoff ML, KellenersSmeets NW, Zur Hausen A, et al. The effect of topical diclofenac $3 \%$ and calcitriol $3 \mu \mathrm{g} / \mathrm{g}$ on superficial basal cell carcinoma (sBCC) and nodular basal cell carcinoma (nBCC): a phase II, randomized controlled trial. Journal of the American Academy of Dermatology 2016;75(1):126-34. [CENTRAL: CN-01342883] [DOI: 10.1016/j.jaad.2016.01.050] 
Choi 2016 \{published data only\}10.1111/jdv.13453

Choi SH, Kim KH, Song KH. Er:YAG ablative fractional laserprimed photodynamic therapy with methyl aminolevulinate as an alternative treatment option for patients with thin nodular basal cell carcinoma: 12-month follow-up results of a randomized, prospective, comparative trial. Journal of the European Academy of Dermatology and Venereology 2015;30(5):783-8. [CENTRAL: CN-01158122] [DOI: 10.1111/ jdv.13453]

\section{Clover 2016 \{published and unpublished data\}}

* Clover AJ, Salwa S, McKiernan J, Buckley C, Bourke M, Kelly EJ, et al. Prospective randomised control trial comparing electrochemotherapy and surgery for the primary treatment of basal cell carcinoma. Melanoma Research 2016;26:e76. [CENTRAL: CN-01212975] [DOI: 10.1097/ CMR.0000000000000285] [EMBASE: 612248066]

Clover AJ, Salwa SP, Bourke MG, McKiernan J, Forde PF, O'Sullivan ST, et al. Electrochemotherapy for the treatment of primary basal cell carcinoma; a randomised control trial comparing electrochemotherapy and surgery with five year follow up. European Journal of Surgical Oncology 2020;46(5):847-54. [CENTRAL: CN-02049463] [PMID: 31862134]

\section{Cornell 1990 \{published data only\}}

Cornell RC, Greenway HT, Tucker SB, Edwards L, Ashworth S, Vance JC, et al. Intralesional interferon therapy for basal cell carcinoma. Journal of the American Academy of Dermatology 1990;23(4 Pt 1):694-700. [CENTRAL: CN-00071018]

\section{Edwards 1990 \{published data only\}}

Edwards L, Tucker SB, Perednia D, Smiles KA, Taylor EL, Tanner DJ, et al. The effects of an intralesional sustained-release formulation of interferon alfa- $2 \mathrm{~b}$ on basal cell carcinoma. Archives of Dermatology 1990;126(8):1029-32. [CENTRAL: CN-00069443]

\section{Eigentler 2007 \{published data only\}}

Eigentler TK, Kamin A, Weide BM, Breuninger H, Caroli UM, Möhrle M, et al. A phase III, randomized, open label study to evaluate the safety and efficacy of imiquimod $5 \%$ cream applied thrice weekly for 8 and 12 weeks in the treatment of low-risk nodular basal cell carcinoma. Journal of the American Academy of Dermatology 2007;57(4):616-21. [CENTRAL: CN-00610509]

\section{Eimpunth 2014 \{published data only\}}

Eimpunth S, Oganesyan G, Hamman MS, Lee RA, Jiang SI, Kim SS. Effectiveness of a 595-nm pulsed dye laser for the treatment of basal cell carcinoma using 1 stacked-pulse session. Journal of the American Academy of Dermatology 2014;70(5 (S1)):AB134. [CENTRAL: CN-01057471]

\section{Ezughah 2008 \{published data only\}}

Ezughah FI, Dawe RS, Ibbotson SH, Fleming CJ. A randomized parallel study to assess the safety and efficacy of two different dosing regimens of $5 \%$ imiquimod in the treatment of superficial basal cell carcinoma. Journal of Dermatological Treatment 2008;19(2):111-7. [CENTRAL: CN-00706567]

\section{Foley 2009a \{published data only\}}

* Foley P, Freeman M, Menter A, Siller G, El-Azhary RA, Gebauer K, et al. Photodynamic therapy with methyl aminolevulinate from primary nodular basal cell carcinoma:results of two randomized studies. International Journal of Dermatology 2009;48(11):1236-45. [CENTRAL: CN-00734005]

Foley P, Freeman M, Siller G, Gebauer K, Murrell D, Barnetson R, et al. A phase III randomized study comparing photodynamic therapy (PDT) using methyl aminolevulinate or placebo cream in nodular basal cell carcinoma (NBCC). Journal of the European Academy of Dermatology and Venereology 2003;17(Suppl 3):231. [CENTRAL: CN-00478536]

\section{Foley 2009b \{published data only\}}

* Foley P, Freeman M, Menter A, Siller G, El-Azhary RA, Gebauer K, et al. Photodynamic therapy with methyl aminolevulinate from primary nodular basal cell carcinoma:results of two randomized studies. International Journal of Dermatology 2009;48(11):1236-45. [CENTRAL: CN-00734005]

Tope WD, Menter A, El Azhary R, Soto P. A comparison of topical MAL-PDT versus placebo-PDT in nodular basal cell carcinoma: results of a North American study. In: 7th Asian Congress of Dermatology incorporating the 5th Regional Conference of Paediatric Dermatology; 2005 Sep 28-Oct 03; Kuala Lumpur, Malaysia. 2005:376. [CENTRAL: CN-00602617]

\section{Garcia-Martin 2011 \{published data only\}}

Garcia-Martin E, Gil-Arribas LM, Idoipe M, Alfaro J, Pueyo V, Pablo LE, et al. Comparison of imiquimod $5 \%$ cream versus radiotherapy as treatment for eyelid basal cell carcinoma. British Journal of Ophthalmology 2011;95(10):1393-6. [CENTRAL: CN-00801928]

\section{Geisse 2002 \{published data only\}}

Geisse JK, Marks R, Owens ML, Andres K, Ginkel AM. Imiquimod $5 \%$ cream for 12 weeks treating superficial BCC. In: The 8th World Congress on Cancer of the Skin; 2001 July 18-21; Zurich, Switzerland. 2001:P58. [CENTRAL: CN-00520431]

* Geisse JK, Rich P, Pandya A, Gross K, Andres K, Ginkel A, et al. Imiquimod $5 \%$ cream for the treatment of superficial basal cell carcinoma: a randomized, vehicle-controlled study. Journal of the American Academy of Dermatology 2002;47(3):390-8. [CENTRAL: CN-00390933]

\section{Geisse 2004 \{published data only\}}

Geisse J, Caro I, Lindholm J, Golitz L, Stampone P, Owens M. Imiquimod 5\% cream for the treatment of superficial basal cell carcinoma: results from two phase III, randomized, vehicle-controlled studies. Journal of the American Academy of Dermatology 2004;50(5):722-33. [CENTRAL: CN-00469536]

\section{Haak 2015 \{published data only\}}

Haak CS, Togsverd-Bo K, Thaysen-Petersen D, Wulf HC, Paasch U, Anderson RR, et al. Fractional laser-mediated photodynamic therapy of high-risk basal cell carcinomas a randomized clinical trial. British Journal of Dermatology 2015;172(1):215-22. [CENTRAL: CN-01039978] 
Hall 1986 \{published data only\}

Hall VL, Leppard BJ, McGill J, Kesseler ME, White JE, Goodwin P. Treatment of basal cell carcinoma: comparison of radiotherapy and cryotherapy. Clinical Radiology 1986;37(1):33-4. [CENTRAL: CN-00478536]

\section{Karsai 2015 \{published data only\}}

Karsai S, Friedl $\mathrm{H}$, Buhck $\mathrm{H}$, Jünger M, Podda $M$. The role of the 595-nm pulsed dye laser in treating superficial basal cell carcinoma: Outcome of a double-blind randomized placebo-controlled trial. British Journal of Dermatology 2015;172(3):677-83. [CENTRAL: CN-01068975]

\section{Kessels 2017 \{published data only\}}

Kessels J, Voeten L, Nelemans P, Cleutjens J, Hillen LM, Mosterd K, et al. Topical sinecatechins, $10 \%$, ointment for superficial basal cell carcinoma: A randomized clinical trial. JAMA Dermatology 2017;153(10):1061-3. [CENTRAL: CN-01420948]

\section{Kessels 2018 \{published data only\}10.1111/bjd.16441}

Kessels JP, Kreukels H, Nelemans PJ, Roozeboom MH, van Pelt $\mathrm{H}$, Mosterd K, et al. Treatment of superficial basal cell carcinoma by topical photodynamic therapy with fractionated 5 -aminolaevulinic acid $20 \%$ vs. two-stage topical methyl aminolaevulinate: results of a randomized controlled trial. British Journal of Dermatology 2018;178(5):1056-63. [CENTRAL: CN-01991773]

\section{Kuijpers 2006 \{published data only\}}

Kuijpers DI, Thissen MR, Thissen CA, Neumann MH. Similar effectiveness of methyl aminolevulinate and 5-aminolevulinate in topical photodynamic therapy for nodular basal cell carcinoma. Journal of Drugs in Dermatology 2006;5(7):642-5. [CENTRAL: CN-00566805]

\section{Kuijpers 2007 \{published data only\}}

* Kuijpers DI, Thissen MR, Berretty PJ, Ideler FH, Nelemans PJ, Neumann MH. Surgical excision versus curettage plus cryosurgery in the treatment of basal cell carcinoma. Dermatologic Surgery 2007;33(5):579-87. [CENTRAL: CN-00588176]

Thissen MR, Nieman FH, Ideler AH, Berretty PJ, Neumann HA. Cosmetic results of cryosurgery versus surgical excision for primary uncomplicated basal cell carcinomas of the head and neck. Dermatologic Surgery 2000;26(8):759-64. [CENTRAL: CN-00298974]

\section{Landthaler 1989 \{published data only\}}

Landthaler M, Braun-Falco O. Use of the TDF factor in soft roentgen radiotherapy [Anwendung des TDF-Faktors in der Röntgenweichstrahlentherapie]. Hautarzt 1989;40(12):774-7. [CENTRAL: CN-00194309] [PMID: 2695500]

\section{Marks 2001 \{published data only\}}

Marks R, Gebauer K, Shumack S, Amies M, Bryden J, Fox TL, et al. Imiquimod $5 \%$ cream in the treatment of superficial basal cell carcinoma: Results of a multicentre 6-week doseresponse trial. Journal of the American Academy of Dermatology 2001;44(5):807-13. [CENTRAL: CN-00326531]
Miller 1997 \{published data only\}

Miller BH, Shavin JS, Cognetta A, Taylor RJ, Salasche S, Korey A, et al. Nonsurgical treatment of basal cell carcinomas with intralesional 5-fluorouracil/epinephrine injectable gel. Journal of the American Academy of Dermatology 1997;36(1):72-7. [CENTRAL: CN-00135753]

\section{Morton 2018 \{published data only\}}

Morton CA, Dominicus R, Radny P, Dirschka T, Hauschild A, Reinhold U, et al. A randomized, multi-national, non-inferiority, phase III trial to evaluate the safety and efficacy of BF-200 ALA gel versus MAL cream in the treatment of non-aggressive basal cell carcinoma with photodynamic therapy (PDT). British Journal of Dermatology 2018;179(2):309-19. [CENTRAL: CN-02000788] [PMID: 29432644]

\section{Mosterd 2008 \{published data only\}}

* Mosterd K, Thissen MR, Nelemans P, Kelleners-Smeets NW, Janssen RL, Broekhof KG, et al. Fractionated 5-aminolaevulinic acid-photodynamic therapy vs. surgical excision in the treatment of nodular basal cell carcinoma: results of a randomized controlled trial. British Journal of Dermatology 2008;159(4):864-70. [CENTRAL: CN-00667266]

Roozeboom MH, Aardoom MA, Nelemans PJ, Thissen MR, Kelleners-Smeets NW, Kuijpers DI, et al. Fractionated 5aminolevulinic acid photodynamic therapy after partial debulking versus surgical excision for nodular basal cell carcinoma: a randomized controlled trial with at least 5-year follow-up. Journal of the American Academy of Dermatology August 2013;69(2):280-7. [CENTRAL: CN-00876800] [DOI: 10.1016/j.jid.2016.03.043]

\section{Punjabi 2008 \{published data only\}}

* Punjabi S, Cook LJ, Kersey P, Marks R, Cerio R. Solasodine glycoalkaloids: a novel topical therapy for basal cell carcinoma. A double-blind, randomized, placebo-controlled, parallel group, multicentre study. International Journal of Dermatology 2008;47(1):78-82. [CENTRAL: CN-00629144]

Punjabi S, Cook LJ, Kersey P, Marks R, Finlay A, Sharp G, et al. A double-blind, multicentre parallel group study of BEC-5 cream in basal cell carcinoma. European Academy of Dermatology \& Venereology 2000;14(Suppl 1):49. [CENTRAL: CN-01616931]

Rhodes 2004 \{published data only\}

* Rhodes LE, de Rie M, Enström Y, Groves R, Morken T, Goulden V, et al. Photodynamic therapy using topical methyl aminolevulinate vs surgery for nodular basal cell carcinoma. Archives of Dermatology 2004;140(1):17-23. [CENTRAL: CN-00460094]

Rhodes LE, de Rie MA, Leifsdottir R, Yu RC, Bachmann I, Goulden V, et al. Five year follow-up of a randomized, prospective trial of topical methyl aminolevulinate photodynamic therapy vs surgery for nodular basal cell carcinoma. Archives of Dermatology 2007;143(9):1131-6. [CENTRAL: CN-00612111]

\section{Rogozinski 1997 \{published data only\}}

Rogozinski TT, Jablonska S, Brzoska IM, Wohr C, Gaus W. Intralesional treatment with recombinant interferon beta is an 
effective alternative for the treatment of basal cell carcinoma. Double-blind, placebo-controlled study. [Doogniskowe podawanie rekombinantowego interferonu beta. Skuteczna alternatywa w leczeniu basalimoma (wyniki podwojnie slepej proby)]. Przeglad Dermatologiczny 1997;84(3):259-63. [CENTRAL: CN-00193051]

\section{Romagosa 2000 \{published data only\}}

Romagosa R, Saap L, Givens M, Salvarrey A, He JL, Hsia SL, et al. A pilot study to evaluate the treatment of basal cell carcinoma with 5-fluorouracil using phosphatidyl choline as a transepidermal carrier. Dermatologic Surgery 2000;26(4):338-40. [CENTRAL: CN-00296475]

\section{Salmanpoor 2012 \{published data only\}}

Salmanpoor R, Motevalli D, Saki N, Saki M. Efficacy of excisional surgery, curettage and combined curettage and electrodessication in treatment of basal cell carcinoma. Iranian Journal of Dermatology 2012;15(2):66-7. [CENTRAL: CN-00908884]

\section{Salmivuori 2019 \{published data only\}}

Salmivuori MK, Grönroos M, Tani T, Pölönen I, Räsänen J, Annala L, et al. Hexylaminolevulinate and aminolevulinic acid nanoemulsion have similar tolerability, initial efficacy and cosmetic outcome as methylaminolevulinate in photodynamic therapy of basal cell carcinoma in a prospective randomized double-blinded trial. Journal of Investigative Dermatology 2019;139(9):S234. [CENTRAL: CN-01978390] [DOI: 10.1016/ j.jid.2019.07.119]

\section{Schulze 2005 \{published data only\}}

Schulze HJ, Cribier B, Requena L, Reifenberger J, Ferrandiz C, Diez G, et al. Imiquimod 5\% cream for the treatment of superficial basal cell carcinoma: results from a randomized vehicle-controlled phase III study in Europe. British Journal of Dermatology 2005;152(5):939-47. [CENTRAL: CN-00513173]

\section{Shumack 2002a \{published data only\}}

Shumack S, Marks R, Amies M, Andres K, Ginkel AM. Imiquimod $5 \%$ cream for 6 weeks treating nodular BCC. 8th World Congress on Cancers of the Skin, Zurich, Switzerland 2001;-:p55. [CENTRAL: CN-00849091]

* Shumack S, Robinson J, Kossard S, Golitz L, Greenway H, Schroeter A, et al. Efficacy of topical 5\% imiquimod cream for the treatment of nodular basal cell carcinoma: comparison of dosing regimens. Archives of Dermatology 2002;138(9):1165-71. [CENTRAL: CN-00398024]

\section{Shumack 2002b \{published data only\}}

Robinson JK, Marks R, Owens ML, Andres K, Ginkel AM. Imiquimod $5 \%$ cream for 12 weeks treating nodular BCC. 8th World Congress on Cancer of the Skin, Zurich, Switzerland 2001;-(-):p57. [CENTRAL: CN-00849091]

* Schumack S, Robinson J, Kossard S, Golitz L, Greenway H, Schroeter, A et al. Efficacy of topical 5\% imiquimod cream for the treatment of nodular basal cell carcinoma. Archives of Dermatology 2002;138(9):1165-71. [CENTRAL: CN-00398024]
Siller 2010 \{published data only\}

Siller G, Rosen R, Freeman M, Welburn P, Katsamas J, Ogbourne SM. PEP005 (Ingenol mebutate) gel for the topical treatment of superficial basal cell carcinoma: results of a randomised phase Ila trial. Australasian Journal of Dermatology 2010;51(2):99-105. [CENTRAL: CN-00771587]

\section{Smeets 2004 \{published data only\}}

Essers BA, Dirksen CD, Nieman FH, Smeets NW, Krekels GA, Prins $\mathrm{MH}$, et al. Cost-effectiveness of Mohs micrographic surgery vs surgical excision for basal cell carcinoma of the face. Archives of Dermatology 2006;142(2):187-94. [CENTRAL: CN-00555278]

Mosterd K, Krekels GA, Nieman FH, Ostertag JU, Essers BA, Dirksen CD, et al. Surgical excision versus Mohs' micrographic surgery for primary and recurrent basal-cell carcinoma of the face: a prospective randomised controlled trial with 5-years' follow-up. Lancet Oncology 2008;9(12):1149-56. [CENTRAL: CN-00665334]

* Smeets NW, Krekels GA, Ostertag JU, Essers BA, Dirksen CD, Nieman FH, et al. Surgical excision vs Mohs' micrographic surgery for basal-cell carcinoma of the face: randomised controlled trial. Lancet 2004;364(9447):1766-72. [CENTRAL: CN-00492695]

Van Loo E, Mosterd K, Krekels GA, Roozeboom MH, Ostertag JU, Dirksen CD, et al. Surgical excision versus Mohs' micrographic surgery for basal cell carcinoma of the face: A randomised clinical trial with 10 year follow-up. European Journal of Cancer 2014;50(17):3011-20. [CENTRAL: CN-01043314]

\section{Soler 2000 \{published data only\}}

Soler AM, Angell-Petersen E, Warloe T, Tausjo J, Steen HB, Moan J, et al. Photodynamic therapy of superficial basal cell carcinoma with 5-aminolevulinic acid with dimethylsulfoxide and ethylendiaminetetraacetic acid: A comparison of two light sources. Photochemistry and Photobiology 2000;71(6):724-9. [CENTRAL: CN-00297518]

\section{Spelman 2014 \{published data only\}}

Rosen R, Freeman M, Zibert JR, Katsamas J, Knudsen KM, Spelman L. Ingenol mebutate $0.05 \%$ gel under occlusion is efficacious in treating superficial basal cell carcinoma. British Journal of Dermatology 2014;171(Suppl 4):50. [CENTRAL: CN-01057057]

\section{Sterry 2002a \{published data only\}}

Sterry W, Bichel J, Andres K, Ginkel AM. Imiquimod 5\% cream for 6 weeks with occlusion treating superficial BCC. In: 8th World Congress on cancers of the skin, Zurich, Switzerland. 2001:61. [CENTRAL: CN-00478768]

Sterry W, Bichel J, Ding L, Ginkel AM. Imiquimod 5\% cream for 6 weeks with occlusion treating nodular BCC. In: 8th World Congress on Cancer of the skin, Zurich, Switzerland. 2001.

* Sterry W, Ruzicka T, Herrera E, Takwale A, Bichel J, Andres K, et al. Imiquimod $5 \%$ cream for the treatment of superficial and nodular basal cell carcinoma: randomized studies comparing low-frequency dosing with and without occlusion. British 
Journal of Dermatology 2002;147(6):1227-36. [CENTRAL: CN-00411766] [PMID: 12452875]

\section{Sterry 2002b \{published data only\}}

Sterry W, Bichel J, Andres K, Ginkel AM. Imiquimod 5\% cream for 6 weeks with occlusion treating superficial BCC. 8th World Congress on cancers of the skin, Zurich, Switzerland 2001;-(-):p61. [CENTRAL: CN-00478768]

Sterry W, Bichel J, Ding L, Ginkel AM. Imiquimod 5\% cream for 6 weeks with occlusion treating nodular BCC. In: 8th World Congress on Cancer of the skin, Zurich, Switzerland. 2001.

* Sterry W, Ruzicka T, Herrera E, Takwale A, Bichel J, Andres K, et al. Imiquimod $5 \%$ cream for the treatment of superficial and nodular basal cell carcinoma: randomized studies comparing low-frequency dosing with and without occlusion. British Journal of Dermatology 2002;147(6):1227-36. [CENTRAL: $\mathrm{CN}-00411766]$

\section{Szeimies 2008 \{published data only\}}

Szeimies RM, Ibbotson S, Murrell DF, Rubel D, Frambach Y, De Berker D, et al. A clinical study comparing methyl aminolevulinate photodynamic therapy and surgery in small superficial basal cell carcinoma $(8-20 \mathrm{~mm})$, with a 12-month follow-up. Journal of the European Academy of Dermatology and Venereology 2008;22(11):1302-11. [CENTRAL: CN-00668334]

\section{Tran 2012 \{published data only\}}

Tran HT, Lee RA, Oganesyan G, Jiang SB. Single treatment of non-melanoma skin cancers using a pulsed-dye laser with stacked pulses. Lasers in Surgery and Medicine 2012;44(6):459-67. [CENTRAL: CN-00969795]

\section{Wang 2001 \{published data only\}}

Wang I, Bendsoe N, Klinteberg CA, Enejder AM, AnderssonEngels S, Svanberg S, et al. Photodynamic therapy vs. cryosurgery of basal cell carcinomas: results of a phase III clinical trial. British Journal of Dermatology 2000;144(4):832-40. [CENTRAL: CN-00326537]

\section{References to studies excluded from this review}

\section{Al-Niaimi 2015 \{published data only\}}

Al-Niaimi F, Sheth N, Kurwa HA, Mallipeddi R. Photodynamic therapy followed by Mohs micrographic surgery compared to mohs micrographic surgery alone for the treatment of basal cell carcinoma: results of a pilot single-blinded randomised controlled trial. Journal of Cutaneous and Aesthetic Surgery 2015;8(2):88-91. [CENTRAL: CN-02012011]

\section{Arits 2014 \{published data only\}}

Arits AH, Spoorenberg E, Mosterd K, Nelemans P, KellenersSmeets NW, Essers BA. Cost-effectiveness of topical imiquimod and fluorouracil vs. photodynamic therapy for treatment of superficial basal-cell carcinoma. British Journal of Dermatology 2014;171(6):1501-07. [CENTRAL: CN-01117058]

\section{Berroeta 2007 \{published data only\}}

Berroeta L, Clark C, Dawe RS, Ibbotson SH, Fleming CJ. A randomized study of minimal curettage followed by topical photodynamic therapy compared with surgical excision for lowrisk nodular basal cell carcinoma. British Journal of Dermatology 2007;157(2):401-3. [CENTRAL: CN-00610206]

\section{Butler 2009 \{published data only\}}

Butler DF, Parekh PK, Lenis A. Imiquimod 5\% cream as adjunctive therapy for primary solitary, nodular basal cell carcinomas before Mohs Micrographic Surgery: a randomized, double blind vehicle-controlled study. Dermatologic Surgery 2009;35(1):24-9. [CENTRAL: CN-00669203]

\section{Čarija 2016 \{published data only\}}

Čarija A, Puizina-Ivić N, Vuković D, Mirić Kovačević L, Čapkun V. Single treatment of low-risk basal cell carcinomas with pulsed dye laser-mediated photodynamic therapy (PDL-PDT) compared with photodynamic therapy (PDT): a controlled, investigatorblinded, intra-individual prospective study. Photodiagnosis and Photodynamic Therapy 2016;16:60-5. [CENTRAL: CN-01342603]

\section{Cho 2013 \{published data only\}}

Cho EA, Moloney FJ, Cai H, Au-Yeung A, China C, Scolyer RA. Safety and tolerability of an intratumorally injected DNAzyme, Dz13, in patients with nodular basal-cell carcinoma: a phase 1 first-in-human trial (DISCOVER). Lancet 2013;381(9880):1835-43.

\section{de Haas 2006 \{published data only\}}

de Haas ER, Kruijt B, Sterenborg HJ, Neumann HM, Robinson DJ. Fractionated illumination significantly improves the response of superficial basal cell carcinoma to aminolevulinic acid photodynamic therapy. Journal of Investigative Dermatology 2006;126(12):2679-86. [CENTRAL: CN-00571166]

Dessinioti 2014 \{published data only\}

Dessinioti C, Plaka M, Stratigos AJ. Vismodegib for the treatment of basal cell carcinoma: results and implications of the ERIVANCE BCC trial. Future Oncology 2014;10(6):927-36.

EudraCT 2013-000092-33 \{unpublished data only\} EduraCT 2013-000092-33. Enhanced efficacy of photodynamic therapy in combination with $5 \%$ imiquimod -a randomised, prospective, observer-blinded study in patients with non melanoma skin cancer. www.clinicaltrialsregister.eu/ctr-search/ search?query=2013-000092-33 (first received 19 September 2013). [2013-000092-33]

\section{Genouw 2018 \{published data only\}}

Genouw E, Verheire B, Ongenae K, De Schepper S, Creytens D, Verhaeghe $\mathrm{E}$, et al. Laser-assisted photodynamic therapy for superficial basal cell carcinoma and Bowen's disease: a randomized intrapatient comparison between a continuous and a fractional ablative $\mathrm{CO}_{2}$ laser mode. Journal of the European Academy of Dermatology and Venereology: JEADV 2018;32(11):1897-905. [PMID: 29633367]

\section{Groselj 2018 \{published data only\}}

Groselj A, Bosnjak M, Strojan P, Krzan M, Cemazar M, Sersa G. Efficiency of electrochemotherapy with reduced bleomycin dose in the treatment of nonmelanoma head and neck skin cancer: Preliminary results. Head \& Neck 2018;40(1):120-5. [CENTRAL: CN-01475261] 
Huang 2004 \{published data only\}

Huang CC, Boyce S, Northington M, Desmond R, Soong S-J. Randomised, controlled surgical trial of preoperative tumour curettage of basal cell carcinoma in Mohs micrographic surgery. Journal of the American Academy of Dermatology 2004;51(4):585-91. [CENTRAL: CN-00497417]

\section{Ibbotson 2018 \{published data only\}}

Ibbotson S, Dawe R, Moseley H, Samuel I, Ferguson J. A randomized, controlled trial of portable compared with conventional photodynamic therapy for superficial nonmelanoma skin cancer. British Journal of Dermatology 2018;179(Suppl 1):100. [CENTRAL: CN-01620045]

\section{Lippert 2013 \{published data only\}}

Lippert J, Šmucler R, Vlk M. Fractional carbon dioxide laser improves nodular basal cell carcinoma treatment with photodynamic therapy with methyl 5-aminolevulinate. Dermatologic Surgery 2013;39(8):1202-8. [CENTRAL: CN-02012012] [PMID: 23725586]

\section{Lu 2017 \{published data only\}}

Lu W, Feng W, Tang Y, Tao X, Pan L. Study of ALA and small dosage of HPD photodynamic therapy (HPD-PDT) in treatment of skin cancer. Biomedical Research (India) 2017;28(14):6476-9. [CENTRAL: CN-01417427]

\section{Lui 2004 \{published data only\}}

Lui H, Hobbs L, Tope WD, Lee PK, Elmets C, Provost N, et al. Photodynamic therapy of multiple nonmelanoma skin cancers with verteporfin and red light-emitting diodes. Archives of Dermatology 2004;140(1):26-32. [CENTRAL: CN-00460095]

\section{Migden 2015 \{published data only\}}

Migden MR, Guminski A, Gutzmer R, Dirix L, Lewis KD, Combemale $P$, et al. Treatment with two different doses of sonidegib in patients with locally advanced or metastatic basal cell carcinoma (BOLT): a multicentre, randomised, double-blind phase 2 trial. Lancet Oncology 2015;16(6):716-28. [CENTRAL: CN-01109355]

\section{NCT01033019 \{unpublished data only\}}

NCT01033019. To evaluate the safety, local tolerability, PK and PD of LDE225 on sporadic superficial and nodular skin basal cell carcinomas (sBCC). clinicaltrials.gov/ct2/show/NCT01033019 (first received 16 December 2009).

\section{Nguyen 2018 \{published data only\}}

Nguyen KP, Knuiman GJ, Blokx WA, Hoogedoorn L, Smits T, Gerritsen MJ. Is a single day patient friendly methyl aminolevulinate photodynamic therapy illumination scheme for superficial basal cell carcinoma feasible? A randomized multicenter pilot trial. Journal of Dermatological Treatment 2019;30(2):194-9. [CENTRAL: CN-01930357] [DOI: $10.1080 / 09546634.2018 .1484558]$

\section{Quirk 2010 \{published data only\}}

Quirk C, Gebauer K, De'Ambrosis B, Slade HB, Meng TC. Sustained clearance of superficial basal cell carcinomas treated with imiquimod cream 5\%: results of a prospective 5-year study. Cutis 2010;85(6):318-24. [PMID: 20666194]

\section{Radiotis 2014 \{published data only\}}

Radiotis G, Roberts N, Czajkowska Z, Khanna M, Korner A. Nonmelanoma skin cancer: disease-specific qualityof-life concerns and distress. Oncology Nursing Forum 2014;41(1):57-65. [PMID: 24368239]

\section{Schleier 2007 \{published data only\}}

Schleier P, Berndt A, Kolossa S, Zenk W, Hyckel P, SchultzeMosgau S. Comparison of aminolevulinic acid (ALA)-thermogelPSDT with methyl-ALA-thermogel-PDT in basal cell carcinoma. Photodiagnosis and Photodynamic Therapy 2007;4(3):197-201. [CENTRAL: CN-01951238]

\section{Sekulic 2012 \{published data only\}}

Sekulic A, Migden MR, Oro AE, Dirix L, Lewis KD, Hainsworth JD, et al. Efficacy and safety of vismodegib in advanced basal-cell carcinoma. New England Journal of Medicine 2012;366(23):2171-9. [PMID: 22670903]

\section{Spencer 2006 \{published data only\}}

Spencer JM. Pilot study of Imiquimod 5\% cream as adjunctive therapy to curettage and electrodesiccation for nodular and basal cell carcinoma. Dermatologic Surgery 2006;32(1):63-9. [CENTRAL: CN-00554023] [PMID: 16393600]

Tang 2012 \{published data only\}

Tang JY, Mackay-Wiggan JM, Aszterbaum M, Yauch RL, Lindgren J, Chang K. Inhibiting the hedgehog pathway in patients with the basal-cell nevus syndrome. New England Journal of Medicine 2012;366(23):2180-8. [CENTRAL: CN-00814383]

\section{van der Geer 2012 \{published data only\}}

Van der Geer S, Martens J, Van Roij J, Brand E, Ostertag JU, Verhaegh ME, et al. Imiquimod 5\% cream as pre-treatment of Mohs Micrographic Surgery for nodular basal cell carcinoma in the face, a prospective randomized controlled study. British Journal of Dermatology 2012;167(1):110-5. [CENTRAL: CN-00856436]

\section{Vijlder 2012 \{published data only\}}

Vijlder HC, Sterenborg HJ, Neumann HA, Robinson DJ, Haas ER. Light fractionation significantly improves the response of superficial basal cell carcinoma to aminolaevulinic acid photodynamic therapy: five-year follow-up of a randomized, prospective trial. Acta Dermato-Venereologica 2012;92(6):641-7. [CENTRAL: CN-00878966]

\section{Wettstein 2013 \{published data only\}}

Wettstein R, Erba P, Itin P, Schaefer DJ, Kalbermatten DF. Treatment of basal cell carcinoma with surgical excision and perilesional interferon-alpha. Journal of Plastic, Reconstructive and Aesthetic Surgery 2013;66(7):912-6. [CENTRAL: CN-00964441]

\section{Zane 2017 \{published data only\}}

Zane C, Facchinetti E, Arisi M, Ortel B, Calzavara-Pinton P. Pulsed $\mathrm{CO} 2$ laser ablation of superficial basal cell of limbs and trunk: a comparative randomized clinical trial with cryotherapy and surgical ablation. Dermatologic Surgery 2017;43(7):920-7. [CENTRAL: CN-01401171] 


\section{References to studies awaiting assessment}

Bunker 2000 \{unpublished data only\}

Bunker C. 5 Fluoracil/Adrenaline injectable gel in basal cell carcinoma. National Research Register.

\section{IRCT 2017030732933 N1 \{unpublished data only\}}

IRCT2017030732933N1. A comparison between efficacy and safety of 585-nanometer pulsed dye laser alone and with cryotherapy in treating superficial basal cell carcinoma. en.irct.ir/trial/25544 (first received 21 May 2017).

\section{ISRCTN 92678315 \{unpublished data only\}}

ISRCTN 92678315. Effect of penetration enhancers in topical photodynamic therapy [Comparison of $20 \%$ Aminolaevulinic acid (ALA) versus $20 \%$ ALA $2 \%$ dimethyl sulphoxide (DMSO) $2 \%$ ethylene diamine tetraacetic acid (EDTA) in topical photodynamic therapy]. doi.org/10.1186/ISRCTN92678315 (first received 8 April 2002).

\section{Kang 2018 \{published data only\}}

Kang K, Han M, Cong M, Chen S, Zhang X, Zhang J. The effects of Ella light power combined with imiquimod cream on basal cell carcinoma. Anti-Tumor Pharmacy 2018;8(2):258-62. [en.cnki.com.cn/Article_en/CJFDTotal-LIYX201802033.htm]

\section{Ma 2018 \{published data only\}}

Ma X, Li L, Ma X, Yang J, He Y. Effect of adriamycin combined with cisplatin on basal cell carcinoma and the level of vascular endothelial growth factor in patients. Anti-tumor Pharmacy 2018;6:951-4.

\section{RPCEC00000147 \{unpublished data only\}}

RPCEC00000147. Efficacy study, randomized, controlled, with CIGB-128-A injected perilesional in basal cell carcinoma in different treatment schedules (InCarbacel-V study). registroclinico.sld.cu/en/trials/RPCEC00000147-En (first received 1 February 2013).

\section{References to ongoing studies}

\section{EudraCT 2016-002255-25 \{unpublished data only\}}

EudraCT 2016-002255-25. Electrochemotherapy compared with radiotherapy for the treatment of basal cell carcinoma. www.clinicaltrialsregister.eu/ctr-search/search? query=2016-002255-25 (first received 12 September 2016). [2016-002255-25]

\section{NCT02242929 \{unpublished data only\}}

NCT02242929. Surgery versus combined treatment with curettage and imiquimod for nodular basal cell carcinoma (SCIN). clinicaltrials.gov/ct2/show/NCT02242929 (first received 17 September 2014).

\section{NCT03573401 \{unpublished data only\}}

NCT03573401. A randomized, double blind, vehicle-controlled multicenter phase III study to evaluate the safety and efficacy of BF-200 ALA (Ameluz ${ }^{\circledR}$ ) and BF-RhodoLED ${ }^{\circledR}$ in the treatment of superficial basal cell carcinoma (SBCC) with photodynamic therapy (PDT). clinicaltrials.gov/ct2/show/NCT03573401 (first received 29 June 2018)

\section{Additional references}

\section{Breuninger 1991}

Breuninger H, Dietz K. Prediction of subclinical tumor infiltration in basal cell carcinoma. Journal of Dermatologic Surgery and Oncology 1991;17(7):574-8.

\section{Campana 2017}

Campana LG, Marconato R, Valpione S, Galuppo S, Alaibac M, Rossi CR, et al. Basal cell carcinoma: 10-year experience with electrochemotherapy. Journal of Translational Medicine 2017;15(1):122.

\section{Chiller 2000}

Chiller K, Passaro D, McCalmont T, Vin-Christian K. Efficacy of curettage before excision in clearing surgical margins of nonmelanoma skin cancer. Archives of Dermatology 2000;136(11):1327-32.

\section{Collier 2018}

Collier NJ, Haylett AK, Wong TH, Morton CA, Ibbotson SH, McKenna KE, et al. Conventional and combination topical photodynamic therapy for basal cell carcinoma: systematic review and meta-analysis. British Journal of Dermatology 2018;179(6):1277-96.

\section{Crowson 2006}

Crowson AN. Basal cell carcinoma: biology, morphology and clinical implications. Modern Pathology 2006;19(S2):S127.

\section{Dessinioti 2010}

Dessinioti C, Antoniou C, Katsambas A, Stratigos AJ. Basal cell carcinoma: what's new under the sun. Photochemistry and Photobiology 2010;86(3):481-91.

\section{Drucker 2018}

Drucker AM, Adam GP, Rofeberg V, Gazula A, Smith B, Moustafa F, et al. Treatments of primary basal cell carcinoma of the skin: a systematic review and network meta-analysis. Annals of Internal Medicine 2018;169(7):456-66.

\section{Dummer 2003}

Dummer R, Urosevic M, Kempf W, Hoek K, Hafner J, Burg G. Imiquimod in basal cell carcinoma: how does it work? British Journal of Dermatology 2003;149:57-8.

\section{Fergusson 2002}

Fergusson D, Aaron SD, Guyatt G, Hébert P. Post-randomisation exclusions: the intention to treat principle and excluding patients from analysis. BMJ 2002;325(7365):652-4.

\section{Franchimont 1982}

Franchimont C, Pierard GE, Cauwenberge D, Andc MD, Lapiere $\mathrm{H}$. Episodic progression and regression of basal cell carcinomas. British Journal of Dermatology 1982;106(3):305-10. 


\section{Fritsch 1998}

Fritsch C, Goerz G, Ruzicka T. Photodynamic therapy in dermatology. Archives of Dermatology 1998;134(2):207-14.

\section{GRADEpro [Computer program]}

McMaster University (developed by Evidence Prime, Inc.) GRADEpro GDT. Hamilton (ON): McMaster University (developed by Evidence Prime, Inc.), accessed 2018. Available at gradepro.org.

\section{Griffiths 2005}

Griffiths RW, Suvarna SK, Stone J. Do basal cell carcinomas recur after complete conventional surgical excision? British Journal of Plastic Surgery 2005;58(6):795-805.

\section{Harris 2001}

Harris RB, Griffith K, Moon TE. Trends in the incidence of nonmelanoma skin cancers in southeastern Arizona. Journal of the American Academy of Dermatology 2001;45(4):528-36.

\section{Higgins 2011}

Higgins JP, Green S, editors. Cochrane Handbook for Systematic Reviews of Interventions Version 5.1.0 [updated March 2011]. The Cochrane Collaboration, 2011. Available from http:// handbook.cochrane.org.

\section{Hruza 1994}

Hruza GJ. Mohs micrographic surgery local recurrences. Journal of Dermatologic Surgery and Oncology 1994;20(9):573-7.

\section{Ibbotson 2016}

Ibbotson S, McKenna K. Principles of photodynamic therapy. In: Rook's Textbook of Dermatology, Ninth Edition. John Wiley \& Sons, Ltd, 2016.

\section{Johnson 1996}

Johnson RL, Rothman AL, Xie J, Goodrich LV, Bare JW, Bonifas JM, et al. Human homolog of patched, a candidate gene for the basal cell nevus syndrome. Science 1996;272(5268):1668-71.

\section{Jung 2011}

Jung DS, Cho HH, Ko HC, Bae YC, Oh CK, Kim MB, et al. Recurrent basal cell carcinoma following ablative laser procedures. Journal of the American Academy of Dermatology 2011;64(4):723-9.

\section{Karagas 1999}

Karagas MR, Greenberg ER, Spencer SK, Stukel TA, Mott LA. Increase in incidence rates of basal cell and squamous cell skin cancer in New Hampshire, USA. International Journal of Cancer 1999;81(4):555-9.

\section{Kelleners-Smeets 2017}

Kelleners-Smeets NW, Mosterd K, Nelemans PJ. Treatment of low-risk basal cell carcinoma. Journal of Investigative Dermatology 2017;137(3):539-40.

\section{Kimyai-Asadi 2005}

Kimyai-Asadi A, Alam M, Goldberg LH, Peterson SR, Silapunt S, Jih MH. Efficacy of narrow-margin excision of well-demarcated primary facial basal cell carcinomas. Journal of the American Academy of Dermatology 2005;53(3):464-8.

\section{Kleinerman 2009}

Kleinerman RA. Radiation-sensitive genetically susceptible pediatric sub-populations. Pediatric Radiology 2009;39(1):27-31.

\section{Lomas 2012}

Lomas A, Leonardi-Bee J, Bath-Hextall F. A systematic review of worldwide incidence of nonmelanoma skin cancer. British Journal of Dermatology 2012;166(5):1069-80.

\section{Madan 2016}

Madan V, Lear JT. Basal cell carcinoma. In: Rook's Textbook of Dermatology, Ninth Edition. John Wiley \& Sons, Ltd, 2016.

\section{Mallon 1996}

Mallon E, Dawber R. Cryosurgery in the treatment of basal cell carcinoma: assessment of one and two freeze-thaw cycle schedules. Dermatologic Surgery 1996;22(10):854-8.

\section{Miller 1991}

Miller SJ. Biology of basal cell carcinoma (part 1). Journal of the American Academy of Dermatology 1991;24(1):1-13.

\section{Moher 2009}

Moher D, Liberati A, Tetzlaff J, Altman DG, Prisma Group. Preferred reporting items for systematic reviews and meta-analyses: the PRISMA statement. PLOS Medicine 2009;6(7):e1000097. [PMID: 19621072]

\section{Mosterd 2013}

Mosterd K, Arits AH, Nelemans PJ, Kelleners-Smeets NW. Aesthetic evaluation after non-invasive treatment for superficial basal cell carcinoma. Journal of the European Academy of Dermatology and Venereology: JEADV 2013;27(5):647-50. [DOI: 10.1111/j.1468-3083.2011.04347.x]

\section{Mulholland 2012}

Mulholland MW, Lillemoe KD, Doherty GM, Maier RV, Simeone DM, Upchurch GR. Greenfield's surgery: Scientific principles \& practice. Lippincott Williams \& Wilkins, 2012.

\section{Muller 2009}

Muller FM, Dawe RS, Moseley H, Fleming CJ. Randomized comparison of Mohs micrographic surgery and surgical excision for small nodular basal cell carcinoma: tissue-sparing outcome. Dermatologic Surgery 2009;35(9):1349-54.

\section{NCIN 2010}

National Cancer Intelligence Network (NCIN). Data Briefing. The importance of skin cancer registration; September 2010. Available at www.ncin.org.uk/publications/data_briefings/ skin_cancer_registration.

\section{Nizamoglu 2016}

Nizamoglu M, Douglas H, McArdle C, Mathew B, Vize C, Matteucci P. Using frozen section margin control technique to manage non-melanomatous skin lesions in high-risk 
sites. Journal of Plastic, Reconstructive \& Aesthetic Surgery 2016;69(5):657-62.

\section{Page 2019}

Page MJ, Higgins JP, Sterne JA. Chapter 13: Assessing risk of bias due to missing results in a synthesis. In: Higgins JP, Thomas J, Chandler J, Cumpston M, Li T, Page MJ, et al (editors). Cochrane Handbook for Systematic Reviews of Interventions version 6.0 (updated July 2019). Cochrane, 2019. Available from www.training.cochrane.org/handbook.

\section{Raasch 2002}

Raasch BA, Buettner PG. Multiple nonmelanoma skin cancer in an exposed Australian population. International Journal of Dermatology 2002;41(10):652-8.

\section{Randle 1996}

Randle HW. Basal cell carcinoma identification and treatment of the high-risk patient. Dermatologic Surgery 1996;22(3):255-61.

\section{RevMan 5.4 [Computer program]}

The Cochrane Collaboration Review Manager (RevMan) [Computer program]. Version 5.4. The Cochrane Collaboration, May 2020. available at https://training.cochrane.org/.

\section{Roewert-Huber 2007}

Roewert-Huber J, Lange-Asschenfeldt B, Stockfleth E, Kerl H. Epidemiology and aetiology of basal cell carcinoma. British Journal of Dermatology 2007;157(s2):47-51.

\section{Rosen 2012}

Rosen RH, Gupta AK, Tyring SK. Dual mechanism of action of ingenol mebutate gel for topical treatment of actinic keratoses: rapid lesion necrosis followed by lesion-specific immune response. Journal of the American Academy of Dermatology 2012;66(3):486-93.

\section{Rowe 1989}

Rowe DE, Carroll RJ, Day CL Jr. Long-term recurrence rates in previously untreated (primary) basal cell carcinoma: implications for patient follow-up. Journal of Dermatologic Surgery and Oncology 1989;15(3):315-28.

\section{Schlessinger 2017}

Schlessinger DI, lyengar S, Yanes AF, Lazaroff JM, GodinezPuig V, Chen BR, et al. Development of a core outcome set for clinical trials in basal cell carcinoma: study protocol for a systematic review of the literature and identification of a core outcome set using a Delphi survey. Trials 2017;18(1):490.

\section{Schulz 1995}

Schulz KF, Chalmers I, Hayes RJ. Empirical evidence of bias. JAMA 1995;273:408-12.

\section{Schulz 2010}

Schulz KF, Altman DG, Moher D. CONSORT 2010 statement: updated guidelines for reporting parallel group randomised trials. BMC Medicine 2010;8(1):18.

\section{Schünemann 2013}

Schünemann H, Brozek J, Oxman A editors. GRADE handbook. The GRADE Working Group, 2013.

\section{Scrivener 2002}

Scrivener Y, Grosshans E, Cribier B. Variations of basal cell carcinomas according to gender, age, location and histopathological subtype. British Journal of Dermatology 2002;147(1):41-7.

\section{Shah 2009}

Shah SM, Konnikov N, Duncan LM, Tannous ZS. The effect of $595 \mathrm{~nm}$ pulsed dye laser on superficial and nodular basal cell carcinomas. Lasers in Surgery and Medicine 2009;41(6):417-22.

\section{Singh 2013}

Singh T, Katiyar SK. Green tea polyphenol,(-)epigallocatechin-3-gallate, induces toxicity in human skin cancer cells by targeting $\beta$-catenin signaling. Toxicology and Applied Pharmacology 2013;273(2):418-24.

\section{Sloan 1990}

Sloan KB, Sherertz EF, McTiernan RG. The effect of 5-fluorouracil on inhibition of epidermal DNA synthesis in vivo: a comparison of the effect of formulations and a prodrug of 5-FU. Archives of Dermatological Research 1990;282(7):484-6.

\section{Telfer 2008}

Telfer NR, Colver GB, Morton CA. Guidelines for the management of basal cell carcinoma. British Journal of Dermatology 2008, 2008;159(1):35-48.

\section{Ting 2005}

Ting PT, Kasper R, Arlette JP. Metastatic basal cell carcinoma: report of two cases and literature review. Journal of Cutaneous Medicine and Surgery 2005;9(1):10-5.

\section{Tucker 2006}

Tucker SB, Polasek JW, Perri AJ, Goldsmith EA. Long-term follow-up of basal cell carcinomas treated with perilesional interferon alfa $2 \mathrm{~b}$ as monotherapy. Journal of the American Academy of Dermatology 2006;54(6):1033-8.

\section{van Delft 2019}

van Delft Lc, Nelemans PJ, Jansen MH, Arits H, Roozeboom MH, Hamid MA, et al. Histologic subtype of treatment failures after noninvasive therapy for superficial basal cell carcinoma: An observational study. Journal of the American Academy of Dermatology 2019, 2019;80(4):1022-8. [DOI: 10.1016/ j.jaad.2018.12.028]

\section{van der Most 2006}

van der Most RG, Himbeck R, Aarons S, Carter SJ, Larma I, Robinson C, et al. Antitumor efficacy of the novel chemotherapeutic agent coramsine is potentiated by cotreatment with $\mathrm{CpG}$-containing oligodeoxynucleotides. Journal of Immunotherapy 2006;29(2):134-42.

\section{Venables 2019}

Venables ZC, Nijsten TA, Wong KF, Autier P, Broggio J, Deas A, et al. Epidemiology of basal and cutaneous squamous cell 
carcinoma in the United Kingdom 2013-2015: a cohort study. British Journal of Dermatology 2019 Mar 12 [Epub ahead of print]. [DOI: 10.1111/bjd.17873]

\section{Verkouteren 2017}

Verkouteren JA, Ramdas KH, Wakkee M, Nijsten T. Epidemiology of basal cell carcinoma: scholarly review. British Journal of Dermatology 2017;177(2):359-72.

\section{Wang 2015}

Wang H, Xu Y, Shi J, Gao X, Geng L. Photodynamic therapy in the treatment of basal cell carcinoma: a systematic review and meta-analysis. Photodermatology, Photoimmunology \& Photomedicine 2015;31(1):44-53.

\section{Wolf 1987}

Wolf DJ, Zitelli JA. Surgical margins for basal cell carcinoma. Archives of Dermatology 1987;123(3):340-4.

\section{Wong 2003}

Wong CS, Strange RC, Lear JT. Basal cell carcinoma. BMJ 2003;327(7418):794-8.

\section{References to other published versions of this review}

\section{Bath-Hextall 2003}

Bath-Hextall F, Bong J, Perkins W, Williams HC. Interventions for basal cell carcinoma of the skin. Cochrane Database of Systematic Reviews 2003, Issue 2. Art. No: CD003412. [DOI: 10.1002/14651858.CD003412]

\section{Bath-Hextall 2007}

Bath-Hextall FJ, Perkins W, Bong J, Williams HC. Interventions for basal cell carcinoma of the skin. Cochrane Database of Systematic Reviews 2007, Issue 1. Art. No: CD003412. [DOI: 10.1002/14651858.CD003412.pub2]

* Indicates the major publication for the study

\section{CHARACTERISTICS OF STUDIES}

Characteristics of included studies [ordered by study ID]

2005-001474-27

\section{Study characteristics}

\begin{tabular}{|c|c|c|}
\hline Methods & \multicolumn{2}{|c|}{ Single-centre, double-blinded, parallel-group, phase Ila RCT. ITT analysis. } \\
\hline Participants & \multicolumn{2}{|c|}{$\begin{array}{l}\text { Denmark. } \\
\text { Inclusion criteria: adults with easily identifiable and treatable HP BCC with clearly defined borders } \\
\text { measuring between } 0.5 \mathrm{~cm} 2 \text { and } 4 \mathrm{~cm} \text {. } \\
\text { Exclusion criteria: } \mathrm{BCC} \text { located on the eyelids, lips, nose, mucosa, or in the anogenital area. } \\
\text { Age: not stated. } \\
\text { Gender: not stated. }\end{array}$} \\
\hline Interventions & \multicolumn{2}{|c|}{$\begin{array}{l}\text { Sample size: } 50 \text { participants. } \\
\text { Period of inclusion: October } 2005 \text { to April } 2008 \text {. } \\
\text { T1: Week } 1 \text { - 8: placebo gel followed by tazarotene gel }(n=25) \text {, if not clear after } 8 \text { weeks, further } 8 \text { week } \\
\text { treatment with Baceca }{ }^{\circledR} \text { gel followed by tazarotene gel. } \\
\text { T2: Week } 1-8: \text { Baceca }^{\circledast} \text { gel followed by Tazarotene gel }(n=25) \text {, if not clear after } 8 \text { weeks, further } 8 \text { week } \\
\text { treatment with Baceca }{ }^{\circledR} \text { gel followed by tazarotene gel. }\end{array}$} \\
\hline Outcomes & \multicolumn{2}{|c|}{$\begin{array}{l}\text { Clinical evaluation at } 8 \text { weeks }+/ \text { - histological assessment. } \\
\text { Response rate at } 12 \text { weeks and histological assessment at } 16 \text { weeks. } \\
\text { Patient-reported and safety measurements. }\end{array}$} \\
\hline Notes & \multicolumn{2}{|c|}{ Sponsored by TopoTarget A/S } \\
\hline \multicolumn{3}{|l|}{ Risk of bias } \\
\hline Bias & Authors' judgement & Support for judgement \\
\hline $\begin{array}{l}\text { Random sequence genera- } \\
\text { tion (selection bias) }\end{array}$ & Unclear risk & Insufficient information to make judgement. \\
\hline
\end{tabular}


2005-001474-27 (Continued)

Allocation concealment Unclear risk Insufficient information to make judgement. (selection bias)

Blinding of participants Low risk Patients were blinded.

and personnel (perfor-

mance bias)

All outcomes

Blinding of outcome as- Low risk Investigators were blinded.

sessment (detection bias)

All outcomes

Incomplete outcome data Low risk Only two participants lost to follow-up.
(attrition bias)

All outcomes

Selective reporting (re- High risk

porting bias)

Study prospectively registered. Complete remission (assessed histologically) 8

or 16 week time points (i.e. after each treatment cycle) were registered as the

time points for the primary outcome but only reported data for response rate

at 3-month follow-up which was not a registered outcome.

Abbade 2015

\section{Study characteristics}

\begin{tabular}{|c|c|}
\hline Methods & Single-centre, randomised, parallel RCT - abstract only. \\
\hline Participants & $\begin{array}{l}\text { Brazil. } \\
\text { Inclusion criteria: primary nodular HP BCC in the head and neck with a diameter up to two inches. } \\
\text { Exclusion criteria: Gorlin syndrome, contraindication to surgical resection or PDT. } \\
\text { Age: not stated. } \\
\text { Gender: not stated. }\end{array}$ \\
\hline Interventions & $\begin{array}{l}\text { Sample size: } 57 \text { participants. } 68 \text { HP nodular BCC. } \\
\text { Period of inclusion: not stated. } \\
\text { T1: Standard protocol MAL-PDT ( } \mathrm{n}=33 \text {, two treatment sessions with 1-week interval, lesions shaved } \\
\text { prior to treatment) } \\
\text { T2: SE ( } \mathrm{n}=35,4 \mathrm{~mm} \text { margin) }\end{array}$ \\
\hline Outcomes & $\begin{array}{l}\text { Clinical response at } 3 \text { months, confirmed histologically if deemed necessary. } \\
\text { Histologically-confirmed relapse at 6,12 and 18+ months. }\end{array}$ \\
\hline Notes & No declarations of interest. \\
\hline Risk of bias & \\
\hline Bias & Authors' judgement Support for judgement \\
\hline
\end{tabular}


Abbade 2015 (Continued)

\begin{tabular}{|c|c|c|}
\hline ra- & Low risk & Quote: "treatment was decided by random drawing." \\
\hline
\end{tabular}

Allocation concealment Unclear risk Insufficient information to make judgement.

(selection bias)

Blinding of participants Unclear risk Insufficient information to make judgement.
and personnel (perfor-
mance bias)
All outcomes

\begin{tabular}{|c|c|c|}
\hline $\begin{array}{l}\text { Blinding of outcome as- } \\
\text { sessment (detection bias) }\end{array}$ & Unclear risk & $\begin{array}{l}\text { Unclear if blinded but we felt that unable to truly blind due to the nature of the } \\
\text { interventions but not clear how this would affect outcomes. }\end{array}$ \\
\hline
\end{tabular}

All outcomes

Incomplete outcome data Unclear risk Insufficient information to make judgement.

(attrition bias)

All outcomes

\begin{tabular}{l}
\hline $\begin{array}{l}\text { Selective reporting (re- } \quad \text { Unclear risk } \\
\text { porting bias) }\end{array}$ \\
\hline
\end{tabular}

Abd El-Naby 2019

\section{Study characteristics}

\begin{tabular}{|c|c|c|}
\hline Methods & \multicolumn{2}{|c|}{ Single-centre, randomised, parallel RCT. } \\
\hline Participants & \multicolumn{2}{|c|}{$\begin{array}{l}\text { Egypt. } \\
\text { Inclusion criteria: HP BCC. } \\
\text { Exclusion criteria: wound healing abnormalities, immunosuppressive or dermatological disease, un- } \\
\text { dergoing chemical peel / dermabrasion / radiotherapy, cutaneous infection. } \\
\text { Age: not stated. } \\
\text { Gender: not stated. }\end{array}$} \\
\hline Interventions & \multicolumn{2}{|c|}{$\begin{array}{l}\text { Sample size: } 22 \text { participants. } \\
\text { Period of inclusion: May } 2015 \text { to May } 2016 \text {. } \\
\text { T1: One stacked PDL session }(n=11) \text {. } \\
\text { T2: Two stacked PDL sessions, two weeks apart }(n=11) \text {. }\end{array}$} \\
\hline Outcomes & \multicolumn{2}{|c|}{$\begin{array}{l}\text { Clinical and histological assessment for recurrence at } 1 \text { month. } \\
\text { Safety and tolerability assessments. } \\
\text { Recurrence at } 6 \text { months (unclear how assessment was made). }\end{array}$} \\
\hline Notes & \multicolumn{2}{|c|}{ There are no conflicts of interest. } \\
\hline \multicolumn{3}{|l|}{ Risk of bias } \\
\hline Bias & Authors' judgement & Support for judgement \\
\hline $\begin{array}{l}\text { Random sequence genera- } \\
\text { tion (selection bias) }\end{array}$ & Low risk & Computer-generated list. \\
\hline $\begin{array}{l}\text { Allocation concealment } \\
\text { (selection bias) }\end{array}$ & Low risk & Sequentially-numbered opaque-sealed envelopes. \\
\hline
\end{tabular}


Abd El-Naby 2019 (Continued)

Blinding of participants Unclear risk No blinding of participants and personnel during the study but unclear if this and personnel (perforwould influence study outcomes.

mance bias)

All outcomes

Blinding of outcome as- Low risk Investigators were blinded.

sessment (detection bias)

All outcomes

Incomplete outcome data Low risk

No loss to follow-up.

(attrition bias)

All outcomes

\begin{tabular}{|c|c|c|}
\hline $\begin{array}{l}\text { Selective reporting (re- } \\
\text { porting bias) }\end{array}$ & Unclear risk & $\begin{array}{l}\text { Study not registered prospectively. All expected outcomes from methods sec- } \\
\text { tion reported. }\end{array}$ \\
\hline
\end{tabular}

Alpsoy 1996

\section{Study characteristics}

\begin{tabular}{|c|c|}
\hline Methods & Single-centre, parallel-group RCT. ITT analysis - abstract only. \\
\hline \multirow[t]{4}{*}{ Participants } & $\begin{array}{l}\text { Turkey. } \\
\text { Inclusion criteria: HP BCC. }\end{array}$ \\
\hline & Exclusion criteria: recurrent lesions, genetic or nevoid conditions, deep tissue involvement. \\
\hline & Age range $38-74$, (mean in years): $\mathrm{T} 1=58.7, \mathrm{~T} 2=63.6, \mathrm{~T} 3=60.3$ \\
\hline & Gender: male $=23$, female $=22$ \\
\hline \multirow[t]{6}{*}{ Interventions } & Total: 45 participants, 45 BCCs. \\
\hline & Period of inclusion: not stated. \\
\hline & Intralesional interferon given three times weekly in all groups (10 injections): \\
\hline & T1: IFN alfa-2a $(n=15)$ \\
\hline & T2: IFN alfa $2 b(n=15)$ \\
\hline & T3: IFN alfa $2 a$ and $2 b(n=15)$ (alfa $2 a$ and alfa $2 b$ injected alternately) \\
\hline
\end{tabular}

Outcomes Clinical and histological clearance at 8 weeks.

Weekly laboratory testing during treatment and at week 1 post-treatment.

AEs.

Notes No declarations of interest.

\section{Risk of bias}

\begin{tabular}{lll}
\hline Bias & Authors' judgement & Support for judgement \\
\hline $\begin{array}{l}\text { Random sequence genera- } \\
\text { tion (selection bias) }\end{array}$ & Unclear risk & Insufficient information to make judgement. \\
\hline
\end{tabular}


Alpsoy 1996 (Continued)
Allocation concealment
Unclear risk
Insufficient information to make judgement. (selection bias)

Blinding of participants

Unclear risk

Insufficient information to make judgement. and personnel (performance bias)

All outcomes

Blinding of outcome as- Unclear risk Insufficient information to make judgement.
sessment (detection bias)
All outcomes

All outcomes

Incomplete outcome data Low risk Complete outcome data given for outcomes.
(attrition bias)

All outcomes

Selective reporting (re- Unclear risk Study not registered prospectively.

porting bias)

Arits 2013

\section{Study characteristics}

\begin{tabular}{|c|c|}
\hline Methods & Multi-centre, single-blinded, parallel group RCT. Modified ITT analysis. \\
\hline \multirow[t]{5}{*}{ Participants } & The Netherlands. \\
\hline & Inclusion criteria: primary, HP sBCC. \\
\hline & $\begin{array}{l}\text { Exclusion criteria: patient taking immunosuppressive drugs, genetic skin cancer disorders, breastfeed- } \\
\text { ing or pregnant, tumour located at high-risk areas of the face, or on the scalp. }\end{array}$ \\
\hline & Age (mean in years): $\mathrm{T} 1=63, \mathrm{~T} 2=62, \mathrm{~T} 3=64$ \\
\hline & Gender: male $=303$, female $=298$ \\
\hline \multirow[t]{5}{*}{ Interventions } & Sample size: 601 participants, 601 sBCC. \\
\hline & Period of inclusion: March 2008 to August 2010. \\
\hline & $\begin{array}{l}\text { T1: MAL-PDT ( } n=202 \text {, two treatment sessions with 1-week interval, surface prepared atraumatically } \\
\text { prior to treatment) }\end{array}$ \\
\hline & T2: Imiquimod 5 days per week for 6 weeks $(n=198)$ \\
\hline & T3: 5-FU twice daily for four weeks $(n=201)$ \\
\hline \multirow[t]{6}{*}{ Outcomes } & Primary outcome measure \\
\hline & $\begin{array}{l}\text { Clinical clearance as assessed by blinded assessor at } 3 \text { and } 12 \text { months post-treatment, confirmed histo- } \\
\text { logically if deemed necessary. }\end{array}$ \\
\hline & Secondary outcome measures \\
\hline & Aesthetic outcome assessed by blinded observer on a four-point scale at 12 months post-treatment. \\
\hline & Compliance according to patient diary \\
\hline & Weekly and during-treatment patient-reported pain and burning according to VAS. \\
\hline
\end{tabular}


Arits 2013 (Continued)

Local adverse reactions according to a four-point scale.

AEs.

\begin{tabular}{ll}
\hline Notes & Funded by a Grant of the Netherlands Organisation for Scientific Research. \\
No declarations of interest.
\end{tabular}

\section{Risk of bias}

\begin{tabular}{|c|c|c|}
\hline Bias & Authors' judgement & Support for judgement \\
\hline $\begin{array}{l}\text { Random sequence genera- } \\
\text { tion (selection bias) }\end{array}$ & Low risk & Telephone randomisation. \\
\hline $\begin{array}{l}\text { Allocation concealment } \\
\text { (selection bias) }\end{array}$ & Low risk & $\begin{array}{l}\text { Computer-generated list using random permuted blocks of six to ensure con- } \\
\text { cealment of allocation. }\end{array}$ \\
\hline $\begin{array}{l}\text { Blinding of participants } \\
\text { and personnel (perfor- } \\
\text { mance bias) } \\
\text { All outcomes }\end{array}$ & Unclear risk & $\begin{array}{l}\text { No blinding of participants and personnel during the study but unclear if this } \\
\text { would influence study outcomes. }\end{array}$ \\
\hline $\begin{array}{l}\text { Blinding of outcome as- } \\
\text { sessment (detection bias) } \\
\text { All outcomes }\end{array}$ & Low risk & Done by one physician blinded to treatment assignment. \\
\hline $\begin{array}{l}\text { Incomplete outcome data } \\
\text { (attrition bias) } \\
\text { All outcomes }\end{array}$ & Unclear risk & $\begin{array}{l}\text { Missing outcome data not balanced in numbers across intervention groups but } \\
\text { unclear if missing outcome data related to the nature of the interventions. }\end{array}$ \\
\hline $\begin{array}{l}\text { Selective reporting (re- } \\
\text { porting bias) }\end{array}$ & Unclear risk & $\begin{array}{l}\text { Retrospectively registered (ISRCTN } 79701845 \text { ). The trial registration stated } \\
\text { cosmetic outcome would be measured by participants and observers at } 3 \text { and } \\
12 \text { months' follow-up using the "Patient and Observer scale" and a "5-point } \\
\text { scale". However they reported an observer-rated 4-point scale at } 12 \text { months } \\
\text { only. The study group showed the 4-point scale had better reproducibility for } \\
\text { non-invasive scars in another study (Mosterd 2013) which may explain why } \\
\text { they switched to this outcome measure. }\end{array}$ \\
\hline
\end{tabular}

Avril 1997

\section{Study characteristics}

\begin{tabular}{|c|c|}
\hline Methods & Single-centre, single-blind, parallel-group RCT. ITT analysis. \\
\hline \multirow[t]{3}{*}{ Participants } & $\begin{array}{l}\text { France. } \\
\text { Inclusion criteria: previously untreated BCC of the face, histologically confirmed, smaller than } 4 \mathrm{~cm} \text { in } \\
\text { diameter. } \\
\text { Exclusion criteria: BCC on scalp or neck. Participants who had total removal of BCC at biopsy, with five } \\
\text { or more BCCs, life expectancy below three years. }\end{array}$ \\
\hline & Age: mean 66 years (SD 12). \\
\hline & Gender: male = "50\%", female = "50\%". \\
\hline \multirow[t]{2}{*}{ Interventions } & Sample size: 347 patients, 347 BCC. \\
\hline & Period of inclusion: February 1982 to November 1988 \\
\hline
\end{tabular}


Avril 1997 (Continued)

T1: SE ( $\mathrm{n}=174,2 \mathrm{~mm}$ margin, with or without frozen section margin control)

T2: radiotherapy ( $n=173$, interstitial brachytherapy, superficial contact therapy or conventional therapy)

\begin{tabular}{ll}
\hline Outcomes & Primary outcome measures: histological clearance at 4 years. \\
& Secondary outcome measures: cosmetic results at 3,6,12,24,36 and 48 months. Assessed by 1) patient \\
using VAS 2) dermatologists rating scars using three-point scale, and 3) three blinded independent as- \\
sessors rating appearance using three-point scale.
\end{tabular}

Notes No declarations of interest.

\section{Risk of bias}

\begin{tabular}{lll}
\hline Bias & Authors' judgement & Support for judgement \\
\hline $\begin{array}{l}\text { Random sequence genera- } \\
\text { tion (selection bias) }\end{array}$ & Unclear risk & Only stated that patients were randomly allocated. \\
\hline $\begin{array}{l}\text { Allocation concealment } \\
\text { (selection bias) }\end{array}$ & Unclear risk & Quote: "sequential sealed envelopes opened by the trial data manager." \\
\hline $\begin{array}{l}\text { Blinding of participants } \\
\text { and personnel (perfor- } \\
\text { mance bias) }\end{array}$ & Unclear risk & Unclear if participants or personnel blinded. \\
$\begin{array}{l}\text { All outcomes } \\
\begin{array}{l}\text { Blinding of outcome as- } \\
\text { sessment (detection bias) }\end{array}\end{array}$ & Unclear risk & $\begin{array}{l}\text { Unable to truly blind due to nature of interventions but unclear if this would } \\
\text { influence study outcomes. For cosmetic outcomes however it was felt there } \\
\text { would be a high-risk of bias. }\end{array}$ \\
\hline $\begin{array}{l}\text { Incomplete outcome data } \\
\text { (attrition bias) }\end{array}$ & Low risk & $\begin{array}{l}\text { No missing outcome data. } \\
\text { All outcomes }\end{array}$ \\
\hline $\begin{array}{l}\text { Selective reporting (re- } \\
\text { porting bias) }\end{array}$ & Unclear risk & $\begin{array}{l}\text { Study not registered prospectively. All expected outcomes from methods sec- } \\
\text { tion reported. }\end{array}$ \\
\hline
\end{tabular}

\section{Basset-Seguin 2008}

\section{Study characteristics}

\begin{tabular}{|c|c|}
\hline Methods & Multi-centre, open, parallel-group RCT. PP analysis. \\
\hline \multirow[t]{4}{*}{ Participants } & Europe. \\
\hline & $\begin{array}{l}\text { Inclusion criteria: BCC size between } 6 \mathrm{~mm} \text { and } 15 \mathrm{~mm} \text { on face } / \text { scalp, }<20 \mathrm{~mm} \text { on neck or }<30 \mathrm{~mm} \text { on } \\
\text { trunk }\end{array}$ \\
\hline & $\begin{array}{l}\text { Exclusion criteria: more than } 10 \text { lesions per patient, pigmented, morphoeaform or infiltrating, xeroder- } \\
\text { ma pigmentosum, porphyria, Gorlin's syndrome, arsenic exposure, allergy to MAL/topical photosensi- } \\
\text { tisers/excipients, participation in another trial within } 30 \text { days, pregnancy or breastfeeding, concomi- } \\
\text { tant treatment with immunosuppressive medication. }\end{array}$ \\
\hline & Age (mean in years): $\mathrm{T} 1=62 . \mathrm{T}=64$. \\
\hline
\end{tabular}




Sample size: 118 participants with 219 sBCC.
Period of inclusion: October 1999 to March 2000
T1: MAL-PDT ( $n=60$, initially debrided, one treatment session only)
T2: cryosurgery $(n=58$, double freeze-thaw cycle, with freeze time of up to 20 seconds and thaw time of
2-3 x freeze time).
Participants with an incomplete response at 3 months received further treatment (T1: 2 further MAL-
PDT sessions; T2: repeat cryosurgery).

Outcomes Clinical clearance at 3 months post-treatment, and at 1,2,3,4, and years, assessed as complete or incomplete resolution by assessor and confirmed histologically.

Cosmetic appearance as assessed by patient ( 3 months, 1 and 2 years) and assessor ( 3 months, 1,2,3,4 and 5 years) using four-point scale.

AEs at each follow-up visit up to 3 months post-treatment graded according to three-point severity scale.

Notes No declarations of interest.

\section{Risk of bias}

\begin{tabular}{|c|c|c|}
\hline Bias & Authors' judgement & Support for judgement \\
\hline $\begin{array}{l}\text { Random sequence genera- } \\
\text { tion (selection bias) }\end{array}$ & Low risk & Computer-generated. \\
\hline $\begin{array}{l}\text { Allocation concealment } \\
\text { (selection bias) }\end{array}$ & Unclear risk & Insufficient information to make judgement. \\
\hline $\begin{array}{l}\text { Blinding of participants } \\
\text { and personnel (perfor- } \\
\text { mance bias) } \\
\text { All outcomes }\end{array}$ & Unclear risk & $\begin{array}{l}\text { No blinding of participants and personnel during the study but unclear if this } \\
\text { would influence study outcomes. }\end{array}$ \\
\hline $\begin{array}{l}\text { Blinding of outcome as- } \\
\text { sessment (detection bias) } \\
\text { All outcomes }\end{array}$ & Unclear risk & Unblinded outcome assessor unclear if this would influence study outcomes. \\
\hline $\begin{array}{l}\text { Incomplete outcome data } \\
\text { (attrition bias) } \\
\text { All outcomes }\end{array}$ & Unclear risk & Per protocol analysis. \\
\hline $\begin{array}{l}\text { Selective reporting (re- } \\
\text { porting bias) }\end{array}$ & High risk & $\begin{array}{l}\text { Study prospectively registered (NCT00469417). Registration stated primary } \\
\text { outcome "number of patients in whom } 75 \% \text { or more of the BCC lesions have } \\
\text { responded completely at } 3 \text { months" but reported number of lesions with a } \\
\text { complete response which was a registered secondary outcome. }\end{array}$ \\
\hline
\end{tabular}

Bath-Hextall 2014

\section{Study characteristics}


Bath-Hextall 2014 (Continued)

Methods Multi-centre, non-inferiority, pragmatic parallel-group RCT. Modified ITT analysis.

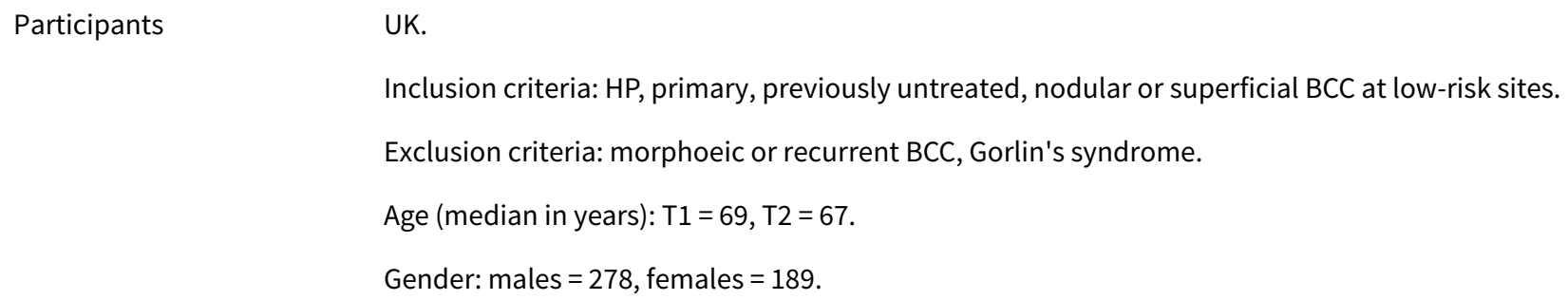

$\begin{array}{ll}\text { Outcomes } & \text { Primary outcome measure } \\ \text { Clinical clearance at } 3 \text { years from start of treatment. } \\ \text { Secondary outcome measures } \\ \text { Clinical clearance at years } 1,2 \text { and } 5 . \\ \text { Time to first failure (stratified). } \\ \text { Cosmetic appearance as rated by participant and dermatologist using six-point scale. } \\ \text { Pain during treatment and at } 16 \text { weeks using six-point scale. } \\ \text { Number of days of pain. } \\ \text { Cost-effectiveness of imiquimod versus surgery. } \\ \text { Funded by Cancer Research UK. } \\ \text { No declarations of interest. }\end{array}$

\section{Risk of bias}

\begin{tabular}{|c|c|c|}
\hline Bias & Authors' judgement & Support for judgement \\
\hline $\begin{array}{l}\text { Random sequence genera- } \\
\text { tion (selection bias) }\end{array}$ & Low risk & Computer-generated block randomisation. \\
\hline $\begin{array}{l}\text { Allocation concealment } \\
\text { (selection bias) }\end{array}$ & Low risk & $\begin{array}{l}\text { Research nurse was required to telephone RDSU to obtain the allocated treat- } \\
\text { ment for the next participant, which concealed the randomisation sequence } \\
\text { from investigators. }\end{array}$ \\
\hline $\begin{array}{l}\text { Blinding of participants } \\
\text { and personnel (perfor- } \\
\text { mance bias) } \\
\text { All outcomes }\end{array}$ & Unclear risk & $\begin{array}{l}\text { No blinding of participants and personnel during the study but unclear if this } \\
\text { would influence study outcomes. }\end{array}$ \\
\hline $\begin{array}{l}\text { Blinding of outcome as- } \\
\text { sessment (detection bias) } \\
\text { All outcomes }\end{array}$ & Unclear risk & Unable to blind but unclear if this will affect judgement of BCC recurrence. \\
\hline
\end{tabular}


Bath-Hextall 2014 (Continued)

Incomplete outcome data Low risk More losses to follow-up in the SE arm (70 vs 48 by 5 years). Modified ITT and (attrition bias) sensitivity analysis were done which found no change to the results.

All outcomes

Selective reporting (re- Low risk Study protocol available and all expected outcomes reported.
porting bias)

\section{Beutner 1999}

\section{Study characteristics}

\begin{tabular}{ll}
\hline Methods & Single-centre, parallel RCT. ITT analysis \\
\hline Participants & USA. \\
& Inclusion criteria: HP BCCS with clearly visible margins for nodular with area of $0.5 \mathrm{~cm}^{2}$ to $1.5 \mathrm{~cm}^{2}$ or su- \\
perficial with an area of $0.5 \mathrm{~cm}^{2}$ to $2 \mathrm{~cm}^{2}$. \\
Exclusion criteria: none described in paper. \\
Age range (years): 37 to 81 \\
Gender: not stated.
\end{tabular}

Interventions

Sample size: 35 participants ( $7 \mathrm{nBCC}, 28 \mathrm{sBCC})$

Period of inclusion: not stated.

Five treatment schedules with the imiquimod $5 \%$ cream or vehicle. All participants continued treatment until either 2 weeks after target tumour was clinically cleared or until 16 weeks.

T1: twice per day $(n=7)$

T2: once per day $(n=4)$

T3: three times per week $(n=4)$

T4: twice per week $(n=5)$

T5: once per week $(n=4)$

T6: vehicle $(n=11)$

Outcomes Complete response rate (histologic clearance of BCC at 6 weeks following treatment), safety evaluations.

Notes Funded by a grant from 3M.

\section{Risk of bias}

\begin{tabular}{lll}
\hline Bias & Authors' judgement & Support for judgement \\
\hline $\begin{array}{l}\text { Random sequence genera- } \\
\text { tion (selection bias) }\end{array}$ & Unclear risk & Insufficient information to make judgement. \\
\hline $\begin{array}{l}\text { Allocation concealment } \\
\text { (selection bias) }\end{array}$ & Unclear risk & Insufficient information to make judgement. \\
\hline $\begin{array}{l}\text { Blinding of participants } \\
\text { and personnel (perfor- } \\
\text { mance bias) }\end{array}$ & Low risk & Quote: "Double-blind" study. \\
\hline
\end{tabular}


Beutner 1999 (Continued)

All outcomes

Blinding of outcome as-
sessment (detection bias) $\quad$ Low risk Quote:"Double-blind" study.

sessment (detection bias)

All outcomes

Incomplete outcome data Low risk Complete outcome data.
(attrition bias)

All outcomes

Selective reporting (re- Unclear risk porting bias)

Study not registered prospectively. All expected outcomes from methods section reported.

Brinkhuizen 2016

\section{Study characteristics}

\begin{tabular}{ll}
\hline Methods & Single-centre, single-blinded, parallel-group RCT. ITT analysis. \\
\hline Participants & The Netherlands. \\
& Inclusion criteria: HP sBCC of $4 \mathrm{~mm}$ or larger. \\
& Exclusion criteria: tumours on the face or scalp, chronic NSAID use or Vitamin D supplementation in the \\
& preceding 30 days. \\
& Age (average in years): $\mathrm{SBCC}: \mathrm{T} 1=63, \mathrm{~T} 2=65.5, \mathrm{~T} 3=67.5, \mathrm{~T} 4=61.5 ; \mathrm{nBCC}: \mathrm{T} 1=78.5, \mathrm{~T} 2=68.6, \mathrm{~T} 3=71$, \\
& $\mathrm{T} 4=66$. \\
& Gender: male $=89$, female $=39$.
\end{tabular}

Interventions

Sample size: total: 128 participants, 128 BCCs

Period of inclusion: November 2011 to February 2013.

Treatment applied twice daily under occlusion for 8 weeks:

T1: diclofenac-3\% gel $(n=32)$

T2: calcitriol 3ug/g ointment $(n=32)$

T3: diclofenac-3\% gel and calcitriol 3ug/g ointment $(n=32)$

T4: no topical treatment $(n=32)$

Outcomes

Primary outcome measure

Post-treatment percentage of cells expressing $\mathrm{Ki}-67$ and $\mathrm{Bcl}-2$, determined by blinded assessor.

Secondary outcome measures

Histologic tumour regression at 8 weeks.

Adverse events (including application site reactions, using VAS) and patient compliance at 8 weeks according to patient diary.

Notes

Funded by the Dutch Cancer Society.

Calcipotriol supplied by Galderma. 
Brinkhuizen 2016 (Continued)

No declarations of interest.

\section{Risk of bias}

\begin{tabular}{|c|c|c|}
\hline Bias & Authors' judgement & Support for judgement \\
\hline $\begin{array}{l}\text { Random sequence genera- } \\
\text { tion (selection bias) }\end{array}$ & Low risk & $\begin{array}{l}\text { Computer-generated random allocation scheme was stratified for histologic } \\
\text { tumour type. }\end{array}$ \\
\hline $\begin{array}{l}\text { Allocation concealment } \\
\text { (selection bias) }\end{array}$ & Low risk & Random permuted blocks of 8 were used to ensure concealment of allocation. \\
\hline $\begin{array}{l}\text { Blinding of participants } \\
\text { and personnel (perfor- } \\
\text { mance bias) } \\
\text { All outcomes }\end{array}$ & Unclear risk & $\begin{array}{l}\text { No blinding of participants and personnel during the study but unclear if this } \\
\text { would influence study outcomes. }\end{array}$ \\
\hline $\begin{array}{l}\text { Blinding of outcome as- } \\
\text { sessment (detection bias) } \\
\text { All outcomes }\end{array}$ & Unclear risk & $\begin{array}{l}\text { Pathologists who assessed histologic tumour regression were blinded. Un- } \\
\text { blinded for rest of the outcomes. }\end{array}$ \\
\hline $\begin{array}{l}\text { Incomplete outcome data } \\
\text { (attrition bias) } \\
\text { All outcomes }\end{array}$ & Unclear risk & Attrition not clearly documented so unable to assess. \\
\hline $\begin{array}{l}\text { Selective reporting (re- } \\
\text { porting bias) }\end{array}$ & Low risk & $\begin{array}{l}\text { Study prospectively registered (NCTO1358045). Primary outcome reported in } \\
\text { publication as stated in register. Secondary outcome reported in publication } \\
\text { was "histologic tumor regression" but this was not a registered secondary out- } \\
\text { come. }\end{array}$ \\
\hline
\end{tabular}

Choi 2016

\section{Study characteristics}

\begin{tabular}{ll} 
Methods & Single-centre, single-blinded, parallel-group RCT. \\
& ITT and PP analysis. \\
\hline Participants & South Korea. \\
& Inclusion criteria: maximum tumour depth of $2 \mathrm{~mm}$, contraindications for surgical excision. \\
& Exclusion criteria: greater than 5 eligible lesions or those located on the midface/nose/periorbital/ocu- \\
& lar, greater than $15 \mathrm{~mm}$, non-nodular subtype, allergy to trial agents, pregnancy or lactation, active sys- \\
& temic infectious disease, immunosuppressive medication, history of melanoma/melasma/keloid, indi- \\
& cation of poor compliance. \\
& Age (mean in years): T1 $=66.9, \mathrm{~T} 2=63.3$. \\
& Gender: male $=21$, female $=18$ \\
& Sample size: 39 patients, $42 \mathrm{nBCC}$ \\
& Inclusion period: March 2011 to September 2012. \\
& T1: Er:YAG AFL MAL-PDT ( $\mathrm{n}=20)$ \\
& T2: MAL-PDT ( $\mathrm{n}=19)$
\end{tabular}


Choi 2016 (Continued)

Outcomes
Primary outcome measures:

Blinded investigator assessment of clinical response rate 3 and 12 months after final treatment.

Recurrence rate at 12 months, based on clinical and histological findings.

Secondary outcome measures:

AEs immediately after treatment, at 1 week, 3 and 12 months.

Investigator assessment of cosmetic outcome at 12 months using a four-point scale.

Tertiary outcome measure:

Patient-reported pain intensity during PDT according to 11-point VAS.

Notes Funded in part by research funds from the Dong-A University.

No declarations of interest.

\section{Risk of bias}

\begin{tabular}{lll}
\hline Bias & Authors' judgement & Support for judgement \\
\hline $\begin{array}{l}\text { Random sequence genera- } \\
\text { tion (selection bias) }\end{array}$ & Low risk & Computer-generated randomisation. \\
\hline $\begin{array}{l}\text { Allocation concealment } \\
\text { (selection bias) }\end{array}$ & Unclear risk & Insufficient information to make judgement. \\
\hline $\begin{array}{l}\text { Blinding of participants } \\
\text { and personnel (perfor- } \\
\text { mance bias) }\end{array}$ & Unclear risk & $\begin{array}{l}\text { Quote: "The patients may have been able to differentiate between the two } \\
\text { treatments because of the characteristics of Er:YAG pre-treatment". Unclear if } \\
\text { this would influence study outcomes. }\end{array}$ \\
\hline $\begin{array}{l}\text { Blinding of outcome as- } \\
\text { sessment (detection bias) }\end{array}$ & Low risk & $\begin{array}{l}\text { Blinded outcome assessors. } \\
\text { All outcomes }\end{array}$ \\
\hline $\begin{array}{l}\text { Incomplete outcome data } \\
\text { (attrition bias) } \\
\text { All outcomes }\end{array}$ & Low risk & Missing outcome data balanced in numbers across intervention groups. \\
\hline $\begin{array}{l}\text { Selective reporting (re- } \\
\text { porting bias) }\end{array}$ & Unclear risk & $\begin{array}{l}\text { Study retrospectively registered (NCT02018679). All expected outcomes from } \\
\text { methods section reported. }\end{array}$ \\
\hline
\end{tabular}

\section{Clover 2016}

\section{Study characteristics}

\begin{tabular}{ll}
\hline Methods & Single-centre, parallel-group, non-inferiority RCT. PP analysis. \\
\hline Participants & Ireland. \\
& Inclusion criteria: superficial, nodular, infiltrative and morphoeic subtypes \\
& $\begin{array}{l}\text { Exclusion criteria: previously treated lesions, advanced BCC, SCC, bleomycin contraindication, chronic } \\
\text { renal failure, coagulopathy, epilepsy, pacemaker, pregnancy or lactation. }\end{array}$
\end{tabular}


Clover 2016 (Continued)

Age (mean in years): T1: 66.8; T2: 63.8

Gender: male $=49$, female $=50$

\begin{tabular}{ll}
\hline Interventions & Sample size: 86 participants; 105 BCCs \\
& Inclusion period: not stated. \\
& T1: electrochemotherapy ( $\mathrm{n}=45$ patients, 60 lesions) \\
& T2: surgical excision ( $\mathrm{n}=41$ participants, 45 lesions) \\
\hline Outcomes & Recurrence rate at $1,2,3$ and 4 years. \\
\hline Notes & No declarations of interest.
\end{tabular}

\section{Risk of bias}

\begin{tabular}{lll}
\hline Bias & Authors' judgement & Support for judgement \\
\hline $\begin{array}{l}\text { Random sequence genera- } \\
\text { tion (selection bias) }\end{array}$ & Low risk & Shuffling of envelopes. \\
\hline $\begin{array}{l}\text { Allocation concealment } \\
\text { (selection bias) }\end{array}$ & Low risk & Sealed envelopes. \\
\hline $\begin{array}{l}\text { Blinding of participants } \\
\text { and personnel (perfor- } \\
\text { mance bias) }\end{array}$ & Unclear risk & $\begin{array}{l}\text { Unblinded but strict protocol and unclear if this would influence study out- } \\
\text { comes. }\end{array}$ \\
\hline $\begin{array}{l}\text { All outcomes } \\
\begin{array}{l}\text { Blinding of outcome as- } \\
\text { sessment (detection bias) }\end{array}\end{array}$ & Unclear risk & $\begin{array}{l}\text { Theoretically blinded outcome assessors but scars may have led to unblind- } \\
\text { ing. }\end{array}$ \\
\hline $\begin{array}{l}\text { Incomplete outcome data } \\
\text { (attrition bias) } \\
\text { All outcomes }\end{array}$ & Unclear risk & $\begin{array}{l}\text { Fairly balanced number of dropouts between groups (19 lesions ECT vs 15 le- } \\
\text { sions SE) but almost 1/5th lost in one group and small overall numbers. }\end{array}$ \\
\hline $\begin{array}{l}\text { Selective reporting (re- } \\
\text { porting bias) }\end{array}$ & Unclear risk & $\begin{array}{l}\text { Study prospectively registered (EUDRACT 2010-019260-37). All expected out- } \\
\text { comes reported but time-points and specific outcomes not stated in trial regis- } \\
\text { ter. }\end{array}$ \\
\hline
\end{tabular}

\section{Cornell 1990}

\section{Study characteristics}

\begin{tabular}{ll}
\hline Methods & Multi-centre, double-blind, parallel-group RCT. PP analysis \\
\hline Participants & USA. \\
Inclusion criteria: HP BCC (superficial or nodular), in good health, between 32 to 70 years of age. \\
Exclusion criteria: previously received therapy to test site, immunosuppressive or cytotoxic therapy \\
(within prior 4 weeks), exogenous interferon/interferon alfa-2b (Intron A), lesion in perioral or central \\
area of the face or penetrating to deep tissue, pregnancy. \\
Age (mean in years): $\mathrm{T} 1=56, \mathrm{~T} 2=57$
\end{tabular}


Cornell 1990 (Continued)

Gender: male $=136$, female $=29$.

Sample size: 172 participants
Inclusion period: not stated.
Both given 3 days per week for 3 consecutive weeks:
T1: Intralesional injections 1.5 million IU of interferon alfa- $2 \mathrm{~b}(\mathrm{n}=123)$
T2: placebo (vehicle for interferon preparation) $(\mathrm{n}=42)$

Outcomes Clinical clearance by blinded assessor using a four-point scale weekly after each of the treatment weeks, then at 5, 9, 13 weeks after completion of treatment, then every 3 months up to 52 weeks.

Adverse events weekly after each of the treatment weeks, then at 5, 9, 13 weeks after completion of treatment, then every 3 months up to 52 weeks.

Assessor- and patient-reported cosmetic outcome at week 16.

Histological assessment of efficacy by blinded assessor at 16 weeks.

Notes Supported by a grant from the Chering Coporation and a grant from Matt and Barbara Browning.

No declarations of interest.

\section{Risk of bias}

\begin{tabular}{|c|c|c|}
\hline Bias & Authors' judgement & Support for judgement \\
\hline $\begin{array}{l}\text { Random sequence genera- } \\
\text { tion (selection bias) }\end{array}$ & Low risk & Randomization quote: "by a computer-generated scheme". \\
\hline $\begin{array}{l}\text { Allocation concealment } \\
\text { (selection bias) }\end{array}$ & Unclear risk & Insufficient information to make judgement. \\
\hline $\begin{array}{l}\text { Blinding of participants } \\
\text { and personnel (perfor- } \\
\text { mance bias) } \\
\text { All outcomes }\end{array}$ & Unclear risk & $\begin{array}{l}\text { Quote: "Double-blind". Use of injection of vehicle in placebo arm however pa- } \\
\text { tients treated with interferon developed systemic symptoms which would like- } \\
\text { ly unblind them. }\end{array}$ \\
\hline $\begin{array}{l}\text { Blinding of outcome as- } \\
\text { sessment (detection bias) } \\
\text { All outcomes }\end{array}$ & Unclear risk & $\begin{array}{l}\text { Total blinding of the examining physician was not possible because systemic } \\
\text { symptoms developed in more patients who received interferon than in the } \\
\text { placebo group. Unclear if this would influence study outcomes. Histopatholo- } \\
\text { gist blinded. }\end{array}$ \\
\hline $\begin{array}{l}\text { Incomplete outcome data } \\
\text { (attrition bias) } \\
\text { All outcomes }\end{array}$ & Unclear risk & $\begin{array}{l}\text { Per protocol analysis. } 7 / 130 \text { vs } 0 / 42 \text { interferon group vs placebo lost to fol- } \\
\text { low-up. }\end{array}$ \\
\hline $\begin{array}{l}\text { Selective reporting (re- } \\
\text { porting bias) }\end{array}$ & Unclear risk & $\begin{array}{l}\text { Study not registered prospectively. All expected outcomes from methods sec- } \\
\text { tion reported. }\end{array}$ \\
\hline
\end{tabular}

\section{Edwards 1990}

\section{Study characteristics}

Methods Single-centre, parallel-group RCT. PP analysis.


Edwards 1990 (Continued)

Participants
USA.

Inclusion criteria: $\mathrm{nBCC}$ or $\mathrm{SBCC} 0.5 \mathrm{~cm}$ to $1.5 \mathrm{~cm}$ diameter, with well-defined borders that could be easily excised.

Exclusion criteria: serious or debilitating illness, history of thromboembolic or cardiovascular disease, radiation therapy to the test site area, history of arsenic ingestion, pregnancy, breast-feeding, immunosuppression as a result of medication or illness and receiving nonsteroidal anti-inflammatory medication. Morphoeic BCC, recurrent cancers, deeply invasive lesions, periorificial tumours and central facial BCC.

Age range (years): 35 to 65

Gender: not stated.
Sample size: 65 participants

Period of inclusion: not stated.

T1: single injection of 10 million IU zinc chelate interferon alfa- $2 b(n=33)$

T2: 1 dose of 10 million IU of zinc chelate interferon alfa-2b per week for 3 weeks $(n=32)$

Outcomes Clinical assessment at weeks 2, 8, 12 and 16.

Histological assessment at 16 weeks.

Notes No declarations of interest.

\section{Risk of bias}

\begin{tabular}{|c|c|c|}
\hline Bias & Authors' judgement & Support for judgement \\
\hline $\begin{array}{l}\text { Random sequence genera- } \\
\text { tion (selection bias) }\end{array}$ & Unclear risk & $\begin{array}{l}\text { Method of randomisation not given. Insufficient information to make judge- } \\
\text { ment. }\end{array}$ \\
\hline $\begin{array}{l}\text { Allocation concealment } \\
\text { (selection bias) }\end{array}$ & Unclear risk & $\begin{array}{l}\text { No information given on allocation concealment. Insufficient information to } \\
\text { make judgement. }\end{array}$ \\
\hline $\begin{array}{l}\text { Blinding of participants } \\
\text { and personnel (perfor- } \\
\text { mance bias) } \\
\text { All outcomes }\end{array}$ & Unclear risk & $\begin{array}{l}\text { Paper does not mention that participants were blinded. Probably not done but } \\
\text { unclear if this would influence study outcomes. }\end{array}$ \\
\hline $\begin{array}{l}\text { Blinding of outcome as- } \\
\text { sessment (detection bias) } \\
\text { All outcomes }\end{array}$ & Unclear risk & $\begin{array}{l}\text { Paper does not mention that outcome assessors were blinded. Probably not } \\
\text { done but unclear if this would influence study outcomes. }\end{array}$ \\
\hline $\begin{array}{l}\text { Incomplete outcome data } \\
\text { (attrition bias) } \\
\text { All outcomes }\end{array}$ & Low risk & Only 2/65 (both from T2) did not complete the study (due to side effects). \\
\hline $\begin{array}{l}\text { Selective reporting (re- } \\
\text { porting bias) }\end{array}$ & Unclear risk & $\begin{array}{l}\text { Study not registered prospectively. All expected outcomes from methods sec- } \\
\text { tion reported. }\end{array}$ \\
\hline
\end{tabular}


Eigentler 2007 (Continued)

Methods Open-label phase III RCT. ITT analysis.

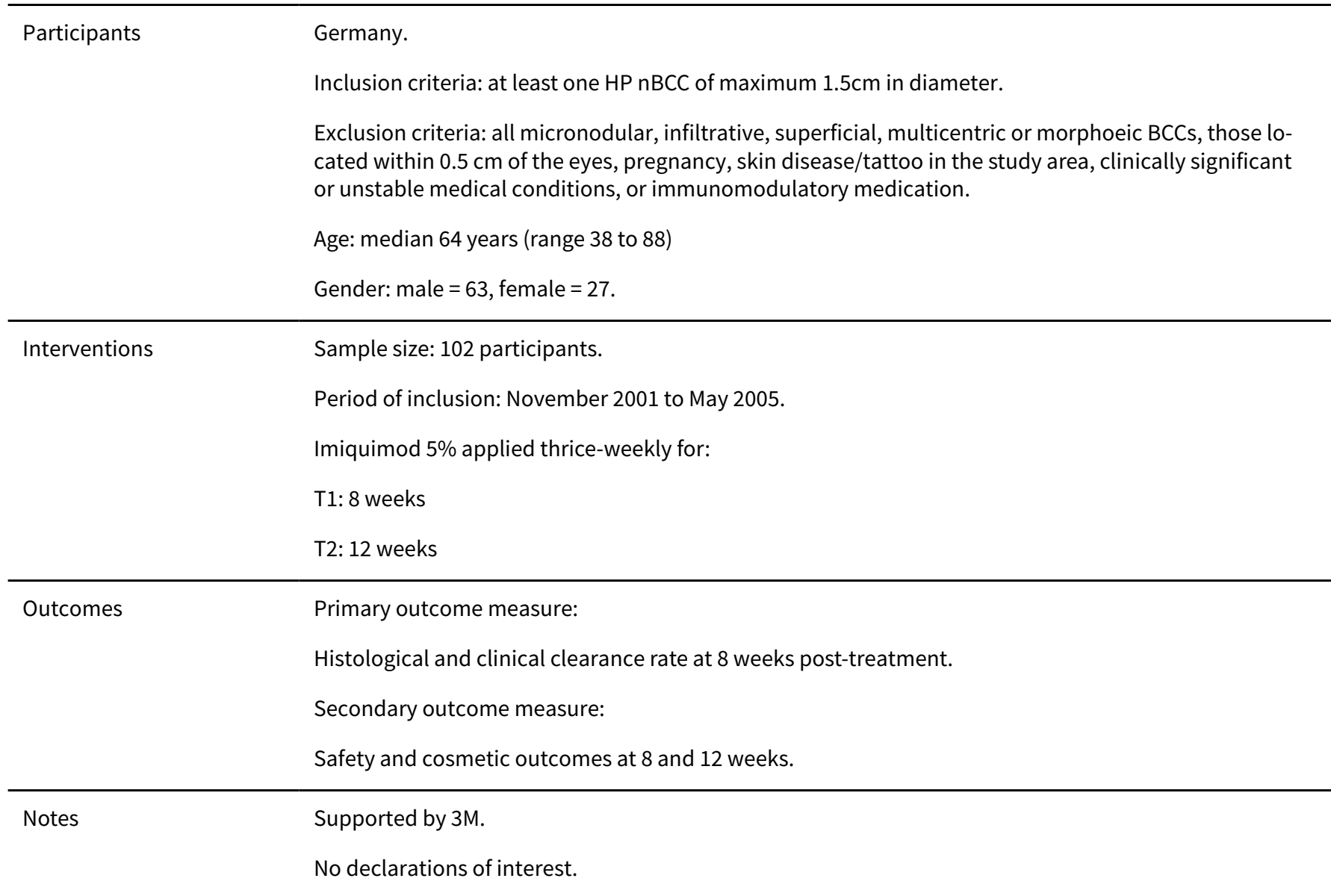

\section{Risk of bias}

\begin{tabular}{lll}
\hline Bias & Authors' judgement & Support for judgement \\
\hline $\begin{array}{l}\text { Random sequence genera- } \\
\text { tion (selection bias) }\end{array}$ & Unclear risk & Insufficient information to make judgement. \\
\hline $\begin{array}{l}\text { Allocation concealment } \\
\text { (selection bias) }\end{array}$ & Unclear risk & Insufficient information to make judgement. \\
\hline $\begin{array}{l}\text { Blinding of participants } \\
\text { and personnel (perfor- } \\
\text { mance bias) } \\
\text { All outcomes }\end{array}$ & Low risk & Open-label, unblinded study but unlikely to influence outcomes. \\
\hline
\end{tabular}

Blinding of outcome as-
sessment (detection bias) $\quad$ Unclear risk $\quad$ Open-label study but unclear if this would influence study outcomes.

All outcomes

\begin{tabular}{|c|c|c|}
\hline $\begin{array}{l}\text { Incomplete outcome data } \\
\text { (attrition bias) }\end{array}$ & Unclear risk & $\begin{array}{l}\text { Losses to follow-up not balanced between groups. Missing outcome data ad } \\
\text { dressed but unclear if related to true outcome. }\end{array}$ \\
\hline
\end{tabular}

All outcomes

Selective reporting (re- Unclear risk
porting bias)

Study retrospectively registered (NCT00204555). All expected outcomes from methods section reported. 
Eimpunth 2014

\section{Study characteristics}

\begin{tabular}{ll}
\hline Methods & Prospective RCT - pilot study, abstract only \\
\hline Participants & USA. \\
Inclusion criteria: HP BCC on trunk or extremities. \\
Exclusion criteria: none described. \\
Age range (years): 29 to 88. \\
Gender: male $=16$, female $=8$. \\
\hline
\end{tabular}

Sample size: 24 participants. $24 \mathrm{HP}$ nodular BCC.
Period of inclusion: not stated.
T1: one session of double-stacked PDL, $10 \mathrm{~mm}$ spot-size, $7.5 \mathrm{~J} / \mathrm{cm} 2$
T2: no treatment

\begin{tabular}{ll}
\hline Outcomes & Histological clearance and immediate / delayed adverse events. \\
\hline Notes & No declarations of interest.
\end{tabular}

\section{Risk of bias}

\begin{tabular}{|c|c|c|}
\hline Bias & Authors' judgement & Support for judgement \\
\hline $\begin{array}{l}\text { Random sequence genera- } \\
\text { tion (selection bias) }\end{array}$ & Unclear risk & Insufficient information to make judgement. \\
\hline $\begin{array}{l}\text { Allocation concealment } \\
\text { (selection bias) }\end{array}$ & Unclear risk & Insufficient information to make judgement. \\
\hline $\begin{array}{l}\text { Blinding of participants } \\
\text { and personnel (perfor- } \\
\text { mance bias) } \\
\text { All outcomes }\end{array}$ & Unclear risk & Insufficient information to make judgement. \\
\hline $\begin{array}{l}\text { Blinding of outcome as- } \\
\text { sessment (detection bias) } \\
\text { All outcomes }\end{array}$ & Unclear risk & Insufficient information to make judgement. \\
\hline $\begin{array}{l}\text { Incomplete outcome data } \\
\text { (attrition bias) } \\
\text { All outcomes }\end{array}$ & Low risk & No losses to follow-up. \\
\hline $\begin{array}{l}\text { Selective reporting (re- } \\
\text { porting bias) }\end{array}$ & Unclear risk & Study retrospectively registered (NCT01245972). \\
\hline
\end{tabular}

\section{Study characteristics}


Ezughah 2008 (Continued)

Methods Single-centre, open-label, parallel RCT. ITT analysis.

Participants UK.

Inclusion criteria: adults with histologically-proven non-invasive and non-infected sBCC with a minimum area and maximum depth of $2 \mathrm{~mm}$.

Exclusion criteria: anogenital tumours, high-risk tumours, nodular/basosquamous/severe squamous metaplastic or infiltrative, Gorlin's syndrome, tumours within $1 \mathrm{~cm}$ of hairline, metastatic/recurrent tumours, involvement in other clinical trials, immunosuppressive treatment, corticosteroid therapy, cryosurgery/cutaneous surgery, pregnant/breastfeeding, other dermatological disorders at target site.

Age: mean 68 (males) and 62 (females)

Gender: male $=17$, female $=13$

Sample size: 32 participants
Period of inclusion: not stated.
Imiquimod $5 \%$ applied:
T1: for 8 weeks with once daily dose for alternate weeks $(n=15)$
T2: for 5 weeks, once daily dose fortnightly with 1-week interval $(n=16)$

\begin{tabular}{ll}
\hline Outcomes & Primary outcome measure: \\
& Clinical clearance at weeks 19 and 52 \\
& Secondary outcome measures: \\
& Weekly patient-reported tolerability using VAS. \\
& Nurse-reported adverse events assessed using four-point severity scale at weeks $2,4,6$ and 8. \\
& AEs also assessed by telephone at weeks $1,3,5$ and 7. \\
Notes & Supported by 3 M. \\
No declarations of interest.
\end{tabular}

\section{Risk of bias}

\begin{tabular}{lll}
\hline Bias & Authors' judgement & Support for judgement \\
\hline $\begin{array}{l}\text { Random sequence genera- } \\
\text { tion (selection bias) }\end{array}$ & Low risk & $\begin{array}{l}\text { Quote: "Computer-generated randomization schedule assigned patients to } \\
\text { each dosing regimen." }\end{array}$ \\
\hline $\begin{array}{l}\text { Allocation concealment } \\
\text { (selection bias) }\end{array}$ & Low risk & A unique identification number in numerical sequence was allocated. \\
\hline $\begin{array}{l}\text { Blinding of participants } \\
\begin{array}{l}\text { and personnel (perfor- } \\
\text { mance bias) }\end{array}\end{array}$ & Unclear risk & Single-blinded but unclear if this would influence performance. \\
All outcomes & \\
\hline
\end{tabular}

\begin{tabular}{|c|c|c|}
\hline $\begin{array}{l}\text { Blinding of outcome as- } \\
\text { sessment (detection bias) } \\
\text { All outcomes }\end{array}$ & Low risk & $\begin{array}{l}\text { Quote:"clinician assessing for clinical clearance at weeks } 19 \text { and } 52 \text { and the } \\
\text { statistician were blinded to the dosing regimen." }\end{array}$ \\
\hline
\end{tabular}


Ezughah 2008 (Continued) Incomplete outcome data Low risk Reasons for missing outcome data unlikely to be related to true outcome. (attrition bias)

Reasons for missing outcome data unlikely to be related to true outcome.

All outcomes

\begin{tabular}{|c|c|c|}
\hline $\begin{array}{l}\text { Selective reporting (re- } \\
\text { porting bias) }\end{array}$ & Unclear risk & $\begin{array}{l}\text { Study not registered prospectively. All expected outcomes from methods sec- } \\
\text { tion reported. }\end{array}$ \\
\hline
\end{tabular}

Foley 2009a

\section{Study characteristics}

$\begin{array}{ll}\text { Methods } & \text { Multi-centre, randomised, double-blind, } \\ \text { placebo-controlled, parallel-group prospective studies performed in Australia (study 308) and the USA } \\ \text { (study 307). ITT analysis. }\end{array}$

(study 307). ITT analysis.

Participants
Inclusion criteria:aAdults with primary HP nodular BCC, suitable for simple excision.
Exclusion criteria: periorbital/perioral/nasal lesions, $<6 \mathrm{~mm},>15 \mathrm{~mm}$ (face or scalp), $>20 \mathrm{~mm}$ (extremi-
ties or neck), $>30 \mathrm{~mm}$ (trunk), pigmented/morphoeaform/infiltrating. Patients with porphyria, xeroder-
ma pigmentosum, arsenic exposure, allergy to study products, recent participation in a different trial,
poorly compliant or pregnant/breastfeeding, immunosuppressive medications.
Age (years): $\mathrm{T} 1=66, \mathrm{~T} 2=67$.
Gender: male $=99$, female $=32$.
Sample size: 66 participants, $75 \mathrm{nBCCs}$.
Period of inclusion: October 2000 to September2002.
Lesion was prepared by gentle tumour surface debridement using a curette prior to application of MAL
or placebo. Number of treatment cycles determined by response:
T1: MAL-PDT ( $\mathrm{n}=66$ participants, 75 lesions)
T2: placebo prior to illumination ( $\mathrm{n}=65$ participants, 75 lesions).
Note these numbers are from both studies combined. Individual study data not presented.

Outcomes Efficacy evaluated histologically by blinded assessor at 3 and 6 months.

Clinical clearance according to a four-point scale.

Safety assessments during treatment, at 2 and 4 weeks, and at 3 months after each treatment cycle.

Assessor- and patient- rated cosmetic outcome according to four-point scale.

Notes $\quad$ Funded byPhotoCure ASA, Oslo, Norway.

The authors declare multiple conflicts of interest.

\section{Risk of bias}

\begin{tabular}{lll}
\hline Bias & Authors' judgement & Support for judgement \\
\hline $\begin{array}{l}\text { Random sequence genera- } \\
\text { tion (selection bias) }\end{array}$ & Low risk & $\begin{array}{l}\text { Computer-generated randomisation scheme prepared by PAREXEL Interna- } \\
\text { tional. Randomisation was stratified by centre. }\end{array}$ \\
\hline
\end{tabular}


Foley 2009a (Continued)

\begin{tabular}{|c|c|c|}
\hline $\begin{array}{l}\text { Allocation concealment } \\
\text { (selection bias) }\end{array}$ & Low risk & Central allocation by PAREXEL. \\
\hline
\end{tabular}

\begin{tabular}{|c|c|c|}
\hline $\begin{array}{l}\text { Blinding of participants } \\
\text { and personnel (perfor- } \\
\text { mance bias) }\end{array}$ & Low risk & $\begin{array}{l}\text { To ensure blinding to treatment, a study nurse was responsible for illumina- } \\
\text { tion and for monitoring adverse events during treatment sessions and at fol- } \\
\text { low-up. }\end{array}$ \\
\hline
\end{tabular}

All outcomes

Blinding of outcome as- Low risk Blinded outcome assessor.

sessment (detection bias)

All outcomes

\begin{tabular}{|c|c|c|}
\hline $\begin{array}{l}\text { Incomplete outcome data } \\
\text { (attrition bias) } \\
\text { All outcomes }\end{array}$ & Low risk & Missing outcome data balanced in numbers between treatment groups. \\
\hline $\begin{array}{l}\text { Selective reporting (re- } \\
\text { porting bias) }\end{array}$ & Unclear risk & $\begin{array}{l}\text { These studies were reported together in the final publication and although } \\
\text { they were very similar in their design, they both had separate trial registra- } \\
\text { tions (NCT00472043 and NCT00472108) and there was no mention of combin- } \\
\text { ing the results of the studies in the trial registers. However it is not clear how } \\
\text { this would affect the overall results. Both studies were retrospectively regis- } \\
\text { tered. }\end{array}$ \\
\hline
\end{tabular}

Foley 2009b

Study characteristics

$\begin{array}{ll}\text { Methods } & \text { Multi-centre, randomised, double-blind, } \\ & \text { placebo-controlled, parallel-group prospective studies performed in Australia (study 308) and the USA } \\ & \text { (study 307). ITT analysis. }\end{array}$

(study 307). ITT analysis.

Participants

USA.

Inclusion criteria: adults with primary HP nodular BCC, suitable for simple excision.

Exclusion criteria: periorbital/perioral/nasal lesions, $<6 \mathrm{~mm},>15 \mathrm{~mm}$ (face or scalp), $>20 \mathrm{~mm}$ (extremities or neck), $>30 \mathrm{~mm}$ (trunk), pigmented/morphoeaform/infiltrating. Patients with porphyria, xeroderma pigmentosum, arsenic exposure, allergy to study products, recent participation in a different trial, poorly compliant or pregnant/breastfeeding, immunosuppressive medications.

Age (years): $\mathrm{T} 1=66, \mathrm{~T} 2=67$.

Gender: male $=99$, female $=32$.

Interventions Sample size: 65 participants, $75 \mathrm{nBCCs}$.

Period of inclusion: October 2000 to September2002.

Lesion was prepared by gentle tumour surface debridement using a curette prior to application of MAL or placebo. Number of treatment cycles determined by response:

T1: MAL-PDT ( $\mathrm{n}=66$ participants, 75 lesions)

T2: placebo prior to illumination ( $n=65$ participants, 75 lesions).

Note these numbers are from both studies combined. Individual study data not presented. 
Foley 2009b (Continued)

Clinical clearance according to a four-point scale.

Safety assessments during treatment, at 2 and 4 weeks, and at 3 months after each treatment cycle.

Assessor- and patient- rated cosmetic outcome according to four-point scale.

\begin{tabular}{ll}
\hline Notes & Funded by PhotoCure ASA, Oslo, Norway. \\
The authors declare multiple conflicts of interest.
\end{tabular}

\section{Risk of bias}

\begin{tabular}{|c|c|c|}
\hline Bias & Authors' judgement & Support for judgement \\
\hline $\begin{array}{l}\text { Random sequence genera- } \\
\text { tion (selection bias) }\end{array}$ & Low risk & $\begin{array}{l}\text { Computer-generated randomisation scheme prepared by PAREXEL Interna- } \\
\text { tional. Randomisation was stratified by centre. }\end{array}$ \\
\hline $\begin{array}{l}\text { Allocation concealment } \\
\text { (selection bias) }\end{array}$ & Low risk & Central allocation by PAREXEL. \\
\hline $\begin{array}{l}\text { Blinding of participants } \\
\text { and personnel (perfor- } \\
\text { mance bias) } \\
\text { All outcomes }\end{array}$ & Low risk & $\begin{array}{l}\text { To ensure blinding to treatment, a study nurse was responsible for illumina- } \\
\text { tion and for monitoring adverse events during treatment sessions and at fol- } \\
\text { low-up }\end{array}$ \\
\hline $\begin{array}{l}\text { Blinding of outcome as- } \\
\text { sessment (detection bias) } \\
\text { All outcomes }\end{array}$ & Low risk & Blinded outcome assessor. \\
\hline $\begin{array}{l}\text { Incomplete outcome data } \\
\text { (attrition bias) } \\
\text { All outcomes }\end{array}$ & Low risk & Missing outcome data balanced in numbers between treatment groups. \\
\hline $\begin{array}{l}\text { Selective reporting (re- } \\
\text { porting bias) }\end{array}$ & Unclear risk & $\begin{array}{l}\text { These studies were reported together in the final publication and although } \\
\text { they were very similar in their design, they both had separate trial registra- } \\
\text { tions (NCT00472043 and NCT00472108) and there was no mention of combin- } \\
\text { ing the results of the studies in the trial registers. However it is not clear how } \\
\text { this would affect the overall results. Both studies were retrospectively regis- } \\
\text { tered. }\end{array}$ \\
\hline
\end{tabular}

Garcia-Martin 2011

\section{Study characteristics}

\begin{tabular}{ll}
\hline Methods & Single-centre, parallel-group RCT. ITT analysis. \\
\hline Participants & Spain. \\
& Inclusion criteria: HP nBCC of the eyelid \\
& Exclusion criteria: previous BCC treatment, concomitant dermatological disease, immunocompro- \\
& mised. \\
& Age: mean 73.7 years (range $53-84)$ \\
Gender: male $=16$, female $=11$.
\end{tabular}

Interventions Sample size: 27 participants


Garcia-Martin 2011 (Continued)

Period of inclusion: not stated.

T1: $5 \%$ imiquimod applied once daily after initial curettage, five times per week for six weeks $(n=15)$

T2: Radiotherapy, standard protocol $(n=12)$

Outcomes
Assessor and patient-evaluated cosmetic outcomes according to a three-point scale at six weeks post-
treatment.
Functional assessment.
Adverse effects and overall tolerability according to three-point rating scale at 3, 6, 12 and 24 months
post-treatment.

Notes No declarations of interest.

\section{Risk of bias}

\begin{tabular}{lll}
\hline Bias & Authors' judgement & Support for judgement \\
\hline $\begin{array}{l}\text { Random sequence genera- } \\
\text { tion (selection bias) }\end{array}$ & High risk & $\begin{array}{l}\text { Randomised to imiquimod if born on even day of month and randomised to RT } \\
\text { if born on odd day of month. }\end{array}$ \\
\hline $\begin{array}{l}\text { Allocation concealment } \\
\text { (selection bias) }\end{array}$ & High risk & Clear risk due to randomisation method. \\
\hline $\begin{array}{l}\text { Blinding of participants } \\
\text { and personnel (perfor- } \\
\text { mance bias) } \\
\text { All outcomes }\end{array}$ & Unclear risk & $\begin{array}{l}\text { Not able to blind participants or personal but unclear if this would influence } \\
\text { performance. }\end{array}$ \\
\hline $\begin{array}{l}\text { Blinding of outcome as- } \\
\text { sessment (detection bias) }\end{array}$ & Unclear risk & Not clear if ophthalmologist was blinded. \\
$\begin{array}{l}\text { All outcomes } \\
\begin{array}{l}\text { Incomplete outcome data } \\
\text { (attrition bias) } \\
\text { All outcomes }\end{array}\end{array}$ & Low risk & None lost to follow-up. \\
\hline $\begin{array}{l}\text { Selective reporting (re- } \\
\text { porting bias) }\end{array}$ & Unclear risk & $\begin{array}{l}\text { Study not registered prospectively. All expected outcomes from methods sec- } \\
\text { tion reported. }\end{array}$ \\
\hline
\end{tabular}

\section{Geisse 2002}

\section{Study characteristics}

Methods Multi-centre phase II, double-blind, parallel-group vehicle-controlled RCT. ITT analysis.

\section{Participants} USA.

Inclusion criteria:aAdults with primary, $\mathrm{HP} \mathrm{sBCC}$ measuring $0.5 \mathrm{~cm}^{\wedge} 2$. to $2.0 \mathrm{~cm}^{\wedge} 2$.

Exclusion criteria: recurrent tumour, within $1 \mathrm{~cm}$ of hairline, eyes, nose, mouth or ears, in the anogenital area or on hands and feet, or within $5 \mathrm{~cm}$ of another BCC tumour.

Age: mean 59 years (range 35 to 85 ) 
Geisse 2002 (Continued)

Gender: male $=82$, female $=46$

Sample size: 128 participants.
Period of inclusion: not stated.
Applied for 12 weeks:
T1: Imiquimod twice daily $(n=10)$
T2: Imiquimod once daily $(n=31)$
T3: Imiquimod 5 consecutive days per week $(n=26)$
T4: Imiquimod 3 days per week $(n=29)$
T5: Vehicle $(n=32)$

Clinical and histological clearance at six weeks post-treatment.
Histological clearance as per two blinded assessors.
Local skin reactions (descriptive and as per a four-point rating scale) and adverse events at each visit.
Total amount of Imiquimod applied
Number of patients taking rest periods.

Notes Supported by 3M.

Multiple authors received funding from or were employed by $3 \mathrm{M}$.

\section{Risk of bias}

\begin{tabular}{lll}
\hline Bias & Authors' judgement & Support for judgement \\
\hline $\begin{array}{l}\text { Random sequence genera- } \\
\text { tion (selection bias) }\end{array}$ & Low risk & Compute- generated randomisation schedule. \\
\hline $\begin{array}{l}\text { Allocation concealment } \\
\text { (selection bias) }\end{array}$ & Unclear risk & Insufficient information to make judgement. \\
\hline $\begin{array}{l}\text { Blinding of participants } \\
\text { and personnel (perfor- } \\
\text { mance bias) }\end{array}$ & Low risk & $\begin{array}{l}\text { Double-blind study. Quote: "Imiquimod cream and vehicle cream were in iden- } \\
\text { tical packaging...Both patients and investigators were blinded to the content } \\
\text { of the treatment cream dispensed". }\end{array}$ \\
\hline $\begin{array}{l}\text { Blinding of outcome as- } \\
\text { sessment (detection bias) }\end{array}$ & Low risk & $\begin{array}{l}\text { Quote: "Biopsy specimens were...read by 2 independent, blinded der- } \\
\text { matopathologists". }\end{array}$ \\
\hline $\begin{array}{l}\text { Incomplete outcome data } \\
\text { (attrition bias) } \\
\text { All outcomes }\end{array}$ & Low risk & \begin{tabular}{l} 
125/128 patients completed study procedures and histological evaluations. \\
\hline $\begin{array}{l}\text { Selective reporting (re- } \\
\text { porting bias) }\end{array}$
\end{tabular} \\
\hline
\end{tabular}


Geisse 2004

\section{Study characteristics}

\begin{tabular}{|c|c|}
\hline Methods & Two identical multicentre phase III randomised, vehicle-controlled studies. ITT analysis. \\
\hline \multirow[t]{4}{*}{ Participants } & $\begin{array}{l}\text { USA. } \\
\text { Inclusion criteria: adults with primary HP sBCC suitable for excision at least } 0.5 \mathrm{~cm}^{2} \text { in size with a maxi- } \\
\text { mum diameter of } 2 \mathrm{~cm} \text {, located on limbs, trunk, neck or head. }\end{array}$ \\
\hline & $\begin{array}{l}\text { Exclusion criteria: lesions located on the facial } \mathrm{H} \text {-zone or in the anogenital area, with no evidence of ag- } \\
\text { gressive growth pattern, dermatological disease in the target site other than BCC and syndromes pre- } \\
\text { disposing to BCCs. }\end{array}$ \\
\hline & Age (years): $\mathrm{T} 1=58.4, \mathrm{~T} 2=59.9, \mathrm{~T} 3=59.4, \mathrm{~T} 4=57.6$ \\
\hline & Gender: male $=445$, female $=279$ \\
\hline
\end{tabular}

Interventions

Sample size: 724 participants.

Period of inclusion: not stated.

For a six week period:

T1: imiquimod $5 \%$ cream 5 days per week

T2: vehicle 5 days per week

T3: imiquimod 5\% cream 7 days per week

T4: vehicle 7 days per week

\section{Outcomes}

Clinical clearance at 12 weeks post-treatment using a four-point rating scale with histological correlation.

Local skin reactions assessed using 4-point rating scale, and adverse reactions at weeks 1,3, and 6 and at post-treatment weeks 4 and 12 .

Supported by $3 \mathrm{M}$.
Multiple authors received funding from or were employed by $3 \mathrm{M}$.

\section{Risk of bias}

\begin{tabular}{lll}
\hline Bias & Authors' judgement & Support for judgement \\
\hline $\begin{array}{l}\text { Random sequence genera- } \\
\text { tion (selection bias) }\end{array}$ & Low risk & Computer-generated randomisation schedule. \\
\hline $\begin{array}{l}\text { Allocation concealment } \\
\text { (selection bias) }\end{array}$ & Low risk & Blocks of four. \\
\hline
\end{tabular}

$\begin{array}{ll}\text { Blinding of participants } & \text { Low risk } \\ \text { and personnel (perfor- } & \text { Quote: "Study personnel and subjects were blinded to the study cream identi- } \\ \text { fication until the database was completed." }\end{array}$

mance bias)

All outcomes

\begin{tabular}{ll}
\hline Blinding of outcome as- & Unclear risk
\end{tabular}

All outcomes

Incomplete outcome data Unclear risk $\quad$ Insufficient information to make judgement.
(attrition bias)


Geisse 2004 (Continued)

All outcomes

\begin{tabular}{|c|c|c|}
\hline $\begin{array}{l}\text { Selective reporting (re- } \\
\text { porting bias) }\end{array}$ & Unclear risk & $\begin{array}{l}\text { Study not registered prospectively. All expected outcomes from methods sec- } \\
\text { tion reported. }\end{array}$ \\
\hline
\end{tabular}

Selective reporting (re porting bias)
Study not registered prospectively. All expected outcomes from methods secreported.

Haak 2015

\section{Study characteristics}

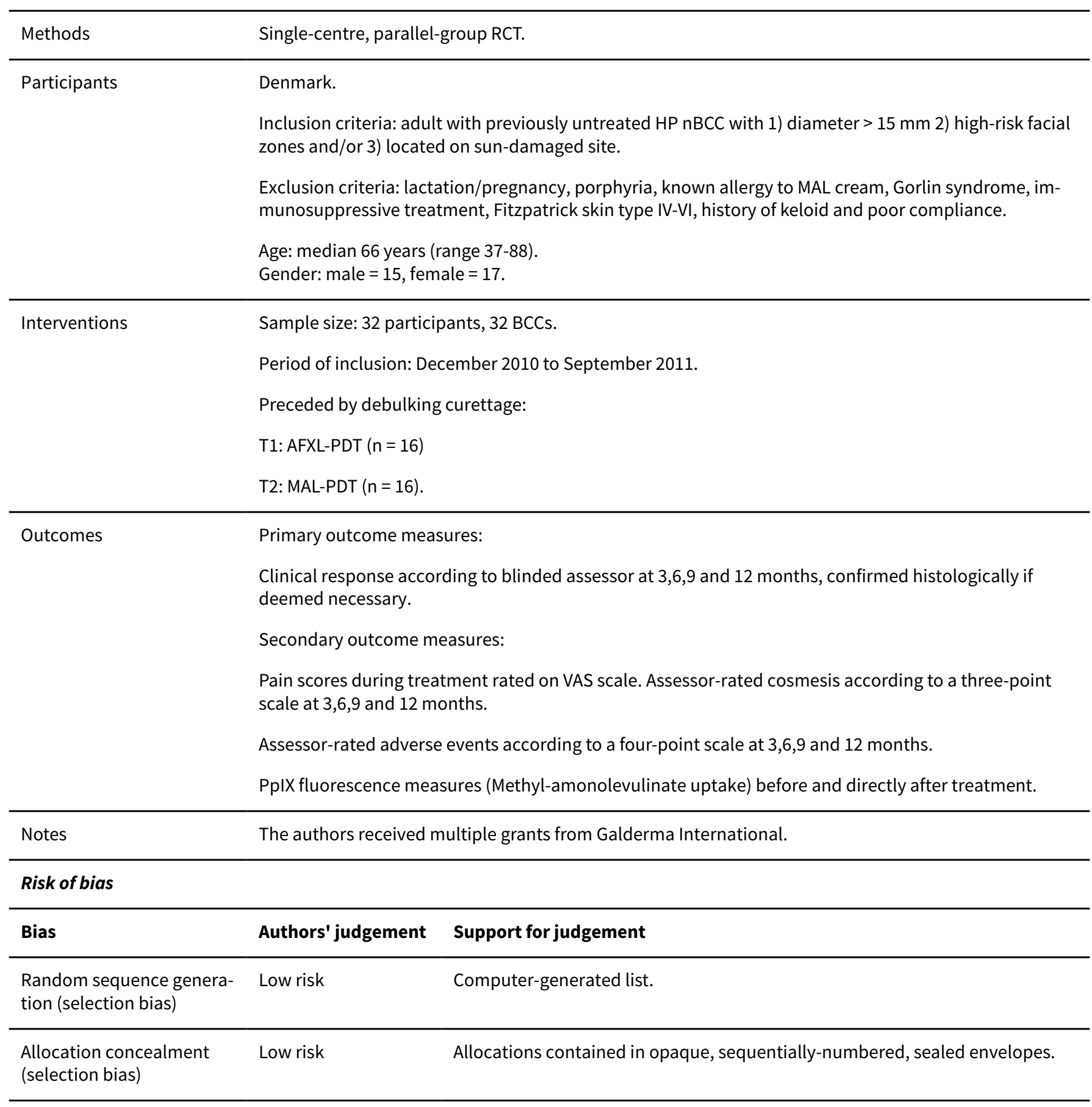


Haak 2015 (Continued)

Blinding of participants Unclear risk Participants and personnel not blinded but unclear if this would influence perand personnel (perforformance. mance bias)

All outcomes

Blinding of outcome as- Low risk Blinded clinical and histological assessments.

sessment (detection bias)

All outcomes

Incomplete outcome data Unclear risk

Unclear reasons for lost to follow-up.

(attrition bias)

u

All outcomes

$\begin{array}{ll}\begin{array}{l}\text { Selective reporting (re- } \\ \text { porting bias) }\end{array} & \text { Low risk } \\ \end{array}$

Hall 1986

\section{Study characteristics}

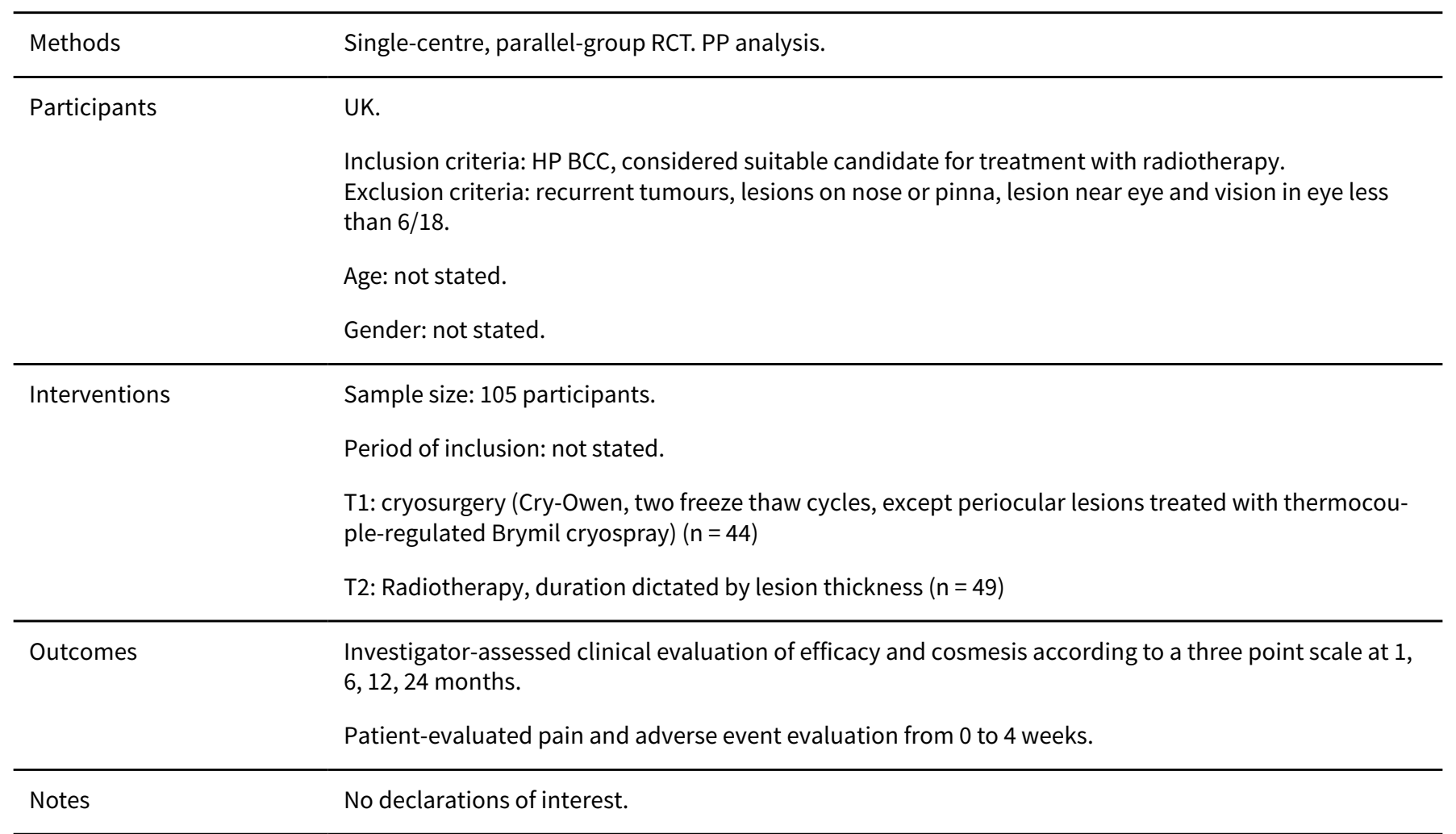

\section{Risk of bias}

\begin{tabular}{lll}
\hline Bias & Authors' judgement & Support for judgement \\
\hline $\begin{array}{l}\text { Random sequence genera- } \\
\text { tion (selection bias) }\end{array}$ & Unclear risk & Insufficient information to make judgement. \\
\hline $\begin{array}{l}\text { Allocation concealment } \\
\text { (selection bias) }\end{array}$ & Unclear risk & Insufficient information to make judgement. \\
\hline
\end{tabular}


Hall 1986 (Continued)

Blinding of participants Unclear risk Insufficient information to make judgement. and personnel (perfor-

mance bias)

All outcomes

Blinding of outcome as-
sessment (detection bias) $\quad$ Unclear risk Insufficient information to make judgement.

All outcomes

Incomplete outcome data Unclear risk Insufficient information to make judgement.

(attrition bias)

All outcomes

\begin{tabular}{|c|c|c|}
\hline $\begin{array}{l}\text { Selective reporting (re- } \\
\text { porting bias) }\end{array}$ & Unclear risk & $\begin{array}{l}\text { Study not registered prospectively. All expected outcomes from methods sec- } \\
\text { tion reported. }\end{array}$ \\
\hline
\end{tabular}

\section{Karsai 2015}

\section{Study characteristics}

\begin{tabular}{ll}
\hline Methods & Multi-centre, parallel-group, double-blinded RCT. \\
\hline Participants & Germany. \\
Inclusion criteria: HP, unpigmented BCC on trunk and extremities, maximum diameter 30 mm. \\
Exclusion criteria: Fitzpatrick skin type $>$ III, pregnancy, previous BCC treatment within 4 weeks, coagu- \\
lation disorders or anticoagulant therapy, photosensitising medications, poor compliance. \\
Age (mean in years): $\mathrm{T} 1=75.3, \mathrm{~T} 2=68.4$. \\
Gender: male $=27$, female $=12$.
\end{tabular}

Interventions

Sample size: 39 participants, 100 sBCCs.

Period of inclusion: November 2012 to October 2013.

T1: PDL $(\mathrm{n}=56)$

T2: sham treatment $(n=44)$.

Outcomes Primary outcome measure:

Clinical and histological remission at 6 months post-treatment.

Secondary outcome measures:

Patient-recorded adverse events and pain intensity graded according to a VAS at 6 weeks and 6 months post-treatment.

Patient satisfaction according to a 3 point scale at 6 weeks and 6 months post-treatment.

Notes Cynosure Multiplex-brand pulsed dye laser provided by Cynosure Inc.

No other conflicts of interests or funding declared.

\section{Risk of bias}


Karsai 2015 (Continued)

\begin{tabular}{|c|c|c|}
\hline Bias & Authors' judgement & Support for judgement \\
\hline $\begin{array}{l}\text { Random sequence genera- } \\
\text { tion (selection bias) }\end{array}$ & Low risk & Randomisation list was generated via a web-based program. \\
\hline $\begin{array}{l}\text { Allocation concealment } \\
\text { (selection bias) }\end{array}$ & Low risk & $\begin{array}{l}\text { Allocation concealed by using sequentially numbered envelopes by the study } \\
\text { nurse. }\end{array}$ \\
\hline $\begin{array}{l}\text { Blinding of participants } \\
\text { and personnel (perfor- } \\
\text { mance bias) } \\
\text { All outcomes }\end{array}$ & Low risk & All blinded except laser practitioner. \\
\hline $\begin{array}{l}\text { Blinding of outcome as- } \\
\text { sessment (detection bias) } \\
\text { All outcomes }\end{array}$ & Unclear risk & $\begin{array}{l}\text { Blinded outcome assessor but residual dyspigmentation after laser treatment } \\
\text { persisted up to } 6 \text { months following treatment so likely to have become un- } \\
\text { blinded. }\end{array}$ \\
\hline $\begin{array}{l}\text { Incomplete outcome data } \\
\text { (attrition bias) } \\
\text { All outcomes }\end{array}$ & Low risk & Quote: "No patients dropped out or lost to follow-up". \\
\hline $\begin{array}{l}\text { Selective reporting (re- } \\
\text { porting bias) }\end{array}$ & Unclear risk & $\begin{array}{l}\text { Study retrospectively registered (NCT02125669). All expected outcomes from } \\
\text { methods section reported. }\end{array}$ \\
\hline
\end{tabular}

\section{Kessels 2017}

\section{Study characteristics}

\begin{tabular}{|c|c|}
\hline Methods & Single-centre, double-blinded, parallel-group, placebo controlled RCT. ITT and PP analysis. \\
\hline \multirow[t]{5}{*}{ Participants } & The Netherlands. \\
\hline & $\begin{array}{l}\text { Inclusion criteria: adults with primary HP sBCC with a diameter between } 4 \mathrm{~mm} \text { and } 20 \mathrm{~mm} \text {, without co- } \\
\text { morbidities interfering with study treatment, and ability to understand instructions. }\end{array}$ \\
\hline & $\begin{array}{l}\text { Exclusion criteria: recurrent BCC, breastfeeding/pregnant, serious co-morbidities, immunosuppression, } \\
\text { genetic skin cancer disorders, high-risk tumour site. }\end{array}$ \\
\hline & Age: not stated. \\
\hline & Gender: not stated. \\
\hline \multirow[t]{5}{*}{ Interventions } & Sample size: 42 participants \\
\hline & Period of inclusion: November 2014 to September 2015. \\
\hline & Applied twice daily for 6 weeks: \\
\hline & T1: topical sinecatechins ointment $10 \%(n=21)$ \\
\hline & T2: placebo ointment $(n=21)$. \\
\hline \multirow[t]{4}{*}{ Outcomes } & Primary outcome measure: \\
\hline & Histological regression as assessed by two blinded investigators at 8 weeks. \\
\hline & Secondary outcome measures: \\
\hline & Patient-reported adverse events at weeks 1,4 , and 6. \\
\hline
\end{tabular}


No conflicts of interest declared.

\section{Risk of bias}

\begin{tabular}{|c|c|c|}
\hline Bias & Authors' judgement & Support for judgement \\
\hline $\begin{array}{l}\text { Random sequence genera- } \\
\text { tion (selection bias) }\end{array}$ & Low risk & $\begin{array}{l}\text { Quote: "a generated randomization list using random permuted blocks of } \\
\text { four". An independent statistician prepared the randomisation list. }\end{array}$ \\
\hline $\begin{array}{l}\text { Allocation concealment } \\
\text { (selection bias) }\end{array}$ & Low risk & $\begin{array}{l}\text { Quote: "The randomization list will not be available to investigators or the } \\
\text { project team." }\end{array}$ \\
\hline $\begin{array}{l}\text { Blinding of participants } \\
\text { and personnel (perfor- } \\
\text { mance bias) } \\
\text { All outcomes }\end{array}$ & Low risk & $\begin{array}{l}\text { Patients and investigators, including the assessing pathologists, blinded to } \\
\text { treatment assignment until the end of the study. Placebo was of identical } \\
\text { colour and consistency as sinecatechins ointment to ensure blinding. }\end{array}$ \\
\hline $\begin{array}{l}\text { Blinding of outcome as- } \\
\text { sessment (detection bias) } \\
\text { All outcomes }\end{array}$ & Low risk & See above. \\
\hline $\begin{array}{l}\text { Incomplete outcome data } \\
\text { (attrition bias) } \\
\text { All outcomes }\end{array}$ & Low risk & Numbers lost to follow-up balanced between groups. \\
\hline $\begin{array}{l}\text { Selective reporting (re- } \\
\text { porting bias) }\end{array}$ & Low risk & $\begin{array}{l}\text { Study prospectively registered (NCT02029352). All expected outcomes report- } \\
\text { ed. }\end{array}$ \\
\hline
\end{tabular}

\section{Kessels 2018}

\section{Study characteristics}

\begin{tabular}{ll}
\hline Methods & Multi-centre, single-blind, parallel-group RCT. ITT and PP analysis. \\
\hline Participants & The Netherlands. \\
Inclusion criteria: adults with primary HP sBCC. \\
Exclusion criteria: immunosuppressive drugs, genetic skin cancer disorder, previous treatment, por- \\
phyria, pregnancy/breastfeeding, known allergy to study materials, tumours located on H-zone on the \\
face, hairy scalp and convex or concave areas such as the ears/fingers. \\
Age (mean in years): T1 $=63.6$, T2 $=65.9$. \\
Gender: male $=75$, female $=87$. \\
Sample size: 162 participants \\
Period of inclusion: September 2013 to May 2015. \\
T1: MAL-PDT ( $\mathrm{n}=80$, standard treatment repeated after 1 week) \\
T2: two-fold fractionated ALA-PDT ( $\mathrm{n}=82$ ). \\
\hline
\end{tabular}


Kessels 2018 (Continued)

Outcomes
Primary outcome measure:

Clinical clearance at 12 months, confirmed histologically if deemed necessary.

Secondary outcome measures:

Aesthetic outcome measured on a 4-point scale and independently scored by two blinded investigators at 3 and 12 months.

Patient-reported adverse events on a 4-point scale 1 week after treatment.

Patient-reported pain on a VAS scale immediately and 1 week after treatment.

Notes

Funded by a grant from the Health Foundation Limburg, the Netherlands.

No conflicts of interests declared.

\section{Risk of bias}

\begin{tabular}{|c|c|c|}
\hline Bias & Authors' judgement & Support for judgement \\
\hline $\begin{array}{l}\text { Random sequence genera- } \\
\text { tion (selection bias) }\end{array}$ & Low risk & Quote: "Computer-generated lists in permuted blocks of six" \\
\hline $\begin{array}{l}\text { Allocation concealment } \\
\text { (selection bias) }\end{array}$ & Low risk & $\begin{array}{l}\text { Quote:"The research physicians did not have access to the randomization } \\
\text { lists". }\end{array}$ \\
\hline $\begin{array}{l}\text { Blinding of participants } \\
\text { and personnel (perfor- } \\
\text { mance bias) } \\
\text { All outcomes }\end{array}$ & Unclear risk & $\begin{array}{l}\text { Quote:"The patients could not be blinded for treatment allocation". Unclear if } \\
\text { this would influence performance. }\end{array}$ \\
\hline $\begin{array}{l}\text { Blinding of outcome as- } \\
\text { sessment (detection bias) } \\
\text { All outcomes }\end{array}$ & Low risk & $\begin{array}{l}\text { Quote:"All study visits... were performed by two investigators who were blind- } \\
\text { ed to treatment allocation". }\end{array}$ \\
\hline $\begin{array}{l}\text { Incomplete outcome data } \\
\text { (attrition bias) } \\
\text { All outcomes }\end{array}$ & Low risk & Numbers lost to follow-up fairly balanced between both groups. \\
\hline $\begin{array}{l}\text { Selective reporting (re- } \\
\text { porting bias) }\end{array}$ & Low risk & $\begin{array}{l}\text { Study registered prospectively (NCT01491711). Primary outcome reported as } \\
\text { described in registration. Secondary outcomes in registration different from } \\
\text { publication (cosmetic outcome and adverse effects reported in publication; } \\
\text { registration reported patient preferences and health care costs as secondary } \\
\text { outcomes). }\end{array}$ \\
\hline
\end{tabular}

\section{Kuijpers 2006}

\section{Study characteristics}

\begin{tabular}{ll}
\hline Methods & Single-centre, parallel RCT - pilot study. \\
\hline Participants & The Netherlands. \\
& $\begin{array}{l}\text { Inclusion criteria:nNodular, primary BCC anywhere except periocular/hairy scalp areas, measuring }<20 \\
\mathrm{~mm} .\end{array}$
\end{tabular}


Kuijpers 2006 (Continued)

Exclusion criteria: pigmented BCC, contraindications to surgery, hypersensitivity to daylight, known allergy to study materials, porphyria, more than $5 \mathrm{BCC}$ per patient.

Age: mean 68.4 years (range $39-87$ ).

Gender: male $=24$, female $=15$

Period of inclusion: not stated.

In each group, half of the tumours were debulked using curettage prior to PDT:

T1: ALA-PDT $(n=22)$

T2: MAL-PDT $(n=21)$

\section{Outcomes}

Histological clearance accorded to blinded assessor at 8 weeks post-treatment.

Pain reported using visual analogue scale and descriptive terms, during and after treatment.

Notes No declarations of interest

\section{Risk of bias}

\begin{tabular}{lll}
\hline Bias & Authors' judgement & Support for judgement \\
\hline $\begin{array}{l}\text { Random sequence genera- } \\
\text { tion (selection bias) }\end{array}$ & Unclear risk & Insufficient information to make judgement. \\
\hline $\begin{array}{l}\text { Allocation concealment } \\
\text { (selection bias) }\end{array}$ & Unclear risk & Insufficient information to make judgement. \\
\hline $\begin{array}{l}\text { Blinding of participants } \\
\text { and personnel (perfor- } \\
\text { mance bias) }\end{array}$ & Low risk & Participants were blinded to allocation. \\
$\begin{array}{l}\text { All outcomes } \\
\text { Blinding of outcome as- } \\
\text { sessment (detection bias) } \\
\text { All outcomes }\end{array}$ & Low risk & Pathologist examining the sections was blinded to treatment. \\
\hline $\begin{array}{l}\text { Incomplete outcome data } \\
\text { (attrition bias) } \\
\text { All outcomes }\end{array}$ & Low risk & No patients dropped out or lost to follow-up. \\
\hline $\begin{array}{l}\text { Selective reporting (re- } \\
\text { porting bias) }\end{array}$ & Unclear risk & Study not registered. All expected outcomes from methods section reported. \\
\hline
\end{tabular}

Kuijpers 2007

\section{Study characteristics}

\begin{tabular}{ll}
\hline Methods & Single-centre, parallel-group RCT. PP analysis. \\
\hline Participants & The Netherlands.
\end{tabular}


Kuijpers 2007 (Continued)

Inclusion criteria: adults with HP primary BCC (nodular or superficial) in the head and neck area, $<20$ $\mathrm{mm}$.

Exclusion criteria: recurrent BCC, other histological subtypes, $>20 \mathrm{~mm}$, contraindications to surgery or cryosurgery, > 5 BCCs.

Age: mean 67 years (range 34-92)

Gender: male $=50$, female $=38$.

Interventions

Sample size: 88 participants, 100 HP BCCs.

Period of inclusion: March 1996 and January 1999.

T1: SE ( $n=49,3$ mm margin)

T2: cryosurgery ( $n=51$, after debulking with curette, using two cycles and cone-spray technique).

\begin{tabular}{ll} 
Outcomes & Histological evidence of recurrence within $5 \mathrm{~mm}$ of the scar at 5 years. \\
& Adverse events. \\
\hline Notes & No declarations of interest.
\end{tabular}

Risk of bias

\begin{tabular}{|c|c|c|}
\hline Bias & Authors' judgement & Support for judgement \\
\hline $\begin{array}{l}\text { Random sequence genera- } \\
\text { tion (selection bias) }\end{array}$ & Low risk & Computer-generated. \\
\hline $\begin{array}{l}\text { Allocation concealment } \\
\text { (selection bias) }\end{array}$ & Low risk & Performed by telephone via uninvolved party \\
\hline $\begin{array}{l}\text { Blinding of participants } \\
\text { and personnel (perfor- } \\
\text { mance bias) } \\
\text { All outcomes }\end{array}$ & Unclear risk & $\begin{array}{l}\text { No blinding of participants and personnel during the study but unclear if this } \\
\text { would influence study outcomes. }\end{array}$ \\
\hline $\begin{array}{l}\text { Blinding of outcome as- } \\
\text { sessment (detection bias) } \\
\text { All outcomes }\end{array}$ & Unclear risk & $\begin{array}{l}\text { No blinding of outcome assessors but unclear if this would influence study } \\
\text { outcomes. For cosmetic outcomes however it was felt there would be a high- } \\
\text { risk of bias. }\end{array}$ \\
\hline $\begin{array}{l}\text { Incomplete outcome data } \\
\text { (attrition bias) } \\
\text { All outcomes }\end{array}$ & Unclear risk & $\begin{array}{l}\text { Difference in numbers lost to follow-up but unclear if due to allocated treat- } \\
\text { ments. }\end{array}$ \\
\hline $\begin{array}{l}\text { Selective reporting (re- } \\
\text { porting bias) }\end{array}$ & Unclear risk & Study not registered. All expected outcomes from methods section reported. \\
\hline
\end{tabular}

Landthaler 1989

\section{Study characteristics}

\begin{tabular}{ll}
\hline Methods & Single-centre, parallel RCT. \\
\hline Participants & Germany.
\end{tabular}


Landthaler 1989 (Continued)

Inclusion criteria: HP BCC and SCC

Exclusion criteria: not stated.

Age: median age 71-75 for both $\mathrm{T} 1$ and $\mathrm{T} 2$.

Gender: T1: 93 = males, $71=$ female; T2: $94=$ male, $61=$ female.

Sample size: 319 participants (BCC and SCC)
Period of inclusion: $1980-1983$
T1: 60 Gy total ( $n=142$ BCCs, 24 SCC, sGy on 20 consecutive days)
T2 48 Gy total ( $n=148$ BCCs, 20 SCCs, 4Gy 3 x/week for 12 sessions).

Outcomes Recurrence rate at 3 years.

Notes No declarations of interest.

\section{Risk of bias}

Bias Authors' judgement Support for judgement

Random sequence genera- Unclear risk Insufficient information to make judgement.

tion (selection bias)

Allocation concealment $\quad$ Unclear risk Insufficient information to make judgement.
(selection bias)

Blinding of participants Unclear risk Insufficient information to make judgement.
and personnel (perfor-
mance bias)
All outcomes

\begin{tabular}{|c|c|c|}
\hline $\begin{array}{l}\text { Blinding of outcome as- } \\
\text { sessment (detection bias) } \\
\text { All outcomes }\end{array}$ & Unclear risk & Insufficient information to make judgement. \\
\hline $\begin{array}{l}\text { Incomplete outcome data } \\
\text { (attrition bias) } \\
\text { All outcomes }\end{array}$ & Low risk & Low numbers lost to follow-up. \\
\hline $\begin{array}{l}\text { Selective reporting (re- } \\
\text { porting bias) }\end{array}$ & Unclear risk & Study not registered. Insufficient information to make judgement. \\
\hline
\end{tabular}

Marks 2001

\section{Study characteristics}

\begin{tabular}{ll}
\hline Methods & Multi-centre, open-label, parallel-group RCT. ITT and PP analysis. \\
\hline Participants & Australia and New Zealand. \\
& $\begin{array}{l}\text { Inclusion criteria: adults with primary HP sBCC on the head, neck, trunk of limbs, with a surface area of } \\
\text { between } 5 \mathrm{~mm}^{2} \text { to } 20 \mathrm{~mm}^{2} .\end{array}$
\end{tabular}


Marks 2001 (Continued)

Exclusion criteria: recurrent, previously treated, within $1 \mathrm{~cm}$ of the hairline/anogenital/facial ori-

fices/distal extremities.

Age: mean 61 years (range 23 to 83 ).

Gender: male $=72$, female $=27$.

Sample size: 99 participants.
Period of inclusion: Not stated.
All groups treated with 5\% imiquimod for six weeks:
T1: twice per day $(n=3)$
T2: once per day $(n=33)$
T3: twice per day for 3 days each week $(n=30)$
T4: once per day for 2 days each week $(n=33)$

Outcomes Histological assessment of efficacy at 6 weeks, and patient-reported adverse events on a three-point scale including characterisation of local reactions.

Notes

Supported by 3M Pharmaceuticals, by whom some authors were employed.

\section{Risk of bias}

\begin{tabular}{lll}
\hline Bias & Authors' judgement & Support for judgement \\
\hline $\begin{array}{l}\text { Random sequence genera- } \\
\text { tion (selection bias) }\end{array}$ & Unclear risk & Insufficient information given to make judgement.
\end{tabular}

tion (selection bias)

$\begin{aligned} & \text { Allocation concealment } \\ & \text { (selection bias) }\end{aligned}$
Unclear risk Insufficient information given to make judgement.

\begin{tabular}{ll}
\hline $\begin{array}{l}\text { Blinding of participants } \\
\text { and personnel (perfor- }\end{array}$ & Unclear risk \\
No blinding of participants and personnel during the study but unclear if this \\
would influence study outcomes.
\end{tabular}
mance bias)

All outcomes

\begin{tabular}{|c|c|c|}
\hline $\begin{array}{l}\text { Blinding of outcome as- } \\
\text { sessment (detection bias) } \\
\text { All outcomes }\end{array}$ & Unclear risk & Insufficient information given to make judgement. \\
\hline
\end{tabular}

Incomplete outcome data Low risk Missing outcome data balanced in numbers between treatment groups.
(attrition bias)

All outcomes

Selective reporting (re- Unclear risk Study not registered. Insufficient information to make judgement.
porting bias)

\section{Miller 1997 \\ Study characteristics}

Methods Multi-centre, open-label, parallel-group RCT. ITT and PP analysis.

Participants USA.


Miller 1997 (Continued)

Inclusion criteria: Single HP BCCs, $6 \mathrm{~mm}$ to $15 \mathrm{~mm}$ in diameter, with well-defined margins, at least 50 $\mathrm{mm}$ away from another malignancy.

Exclusion criteria: lesions that had already received treatment, those at high-risk sites (e.g. eyelids, nose, ears and central part of the face), tumours considered to be more appropriately treated with Mohs micrographic surgery, lesions with deep tissue involvement, morphoeic lesions, basal cell nevus syndrome, hypersensitivities or allergies to 5-FU, sulphites, epinephrine, bovine collagen, history of autoimmune disease or immunosuppression, pregnant or lactating women.

Age: Mean 61 yrs (range 29 - 86).

Gender: male $=97$ male and female $=25$.

Sample size: 122 participants.
Period of inclusion: Not stated.
Intralesional sustained-release 6fluorouracil/epinephrine injectable gel (5FU/epi gel)
T1: $1.0 \mathrm{ml} 5$ FU/epi gel once weekly for 6 weeks $(n=20)$
T2: $0.5 \mathrm{ml}$ 5-FU/epi gel once weekly for 6 weeks $(n=21)$
T3: $1.0 \mathrm{ml} 5$-FU/epi gel twice weekly for 3 weeks $(n=18)$
T4: $0.5 \mathrm{ml} \mathrm{5-FU/epi} \mathrm{gel} \mathrm{twice} \mathrm{weekly} \mathrm{for} 3$ weeks $(n=19)$
T5: $0.5 \mathrm{ml} 5$-FU/epi gel twice weekly for 4 weeks $(n=21)$
T6: $0.5 \mathrm{ml}$ 5-FU/epi gel 3 times weekly for 2 weeks $(n=17)$

Outcomes

Clinical investigator-led examinations at 1, 4, 8 and 12 weeks. Patient-reported assessment of adverse effects at 1, 4, 8 and 12 weeks. Participant- and investigator-reported evaluation of cosmetic appearance according to a five-point scale. Clinical and histological regression assessed at 12 weeks.

\begin{tabular}{|c|c|c|}
\hline Notes & \multicolumn{2}{|c|}{ No declarations of interest } \\
\hline \multicolumn{3}{|l|}{ Risk of bias } \\
\hline Bias & Authors' judgement & Support for judgement \\
\hline $\begin{array}{l}\text { Random sequence genera- } \\
\text { tion (selection bias) }\end{array}$ & Unclear risk & Insufficient information given to make judgement. \\
\hline $\begin{array}{l}\text { Allocation concealment } \\
\text { (selection bias) }\end{array}$ & Unclear risk & Insufficient information given to make judgement. \\
\hline $\begin{array}{l}\text { Blinding of participants } \\
\text { and personnel (perfor- } \\
\text { mance bias) } \\
\text { All outcomes }\end{array}$ & Unclear risk & $\begin{array}{l}\text { Study does not mention blinding of participants and personnel during the } \\
\text { study but unclear if this would influence study outcomes. }\end{array}$ \\
\hline $\begin{array}{l}\text { Blinding of outcome as- } \\
\text { sessment (detection bias) } \\
\text { All outcomes }\end{array}$ & Unclear risk & $\begin{array}{l}\text { Study does not mention blinding of participants and personnel during the } \\
\text { study but unclear if this would influence study outcomes. }\end{array}$ \\
\hline $\begin{array}{l}\text { Incomplete outcome data } \\
\text { (attrition bias) } \\
\text { All outcomes }\end{array}$ & Unclear risk & Most participants completed the study (116/122). \\
\hline
\end{tabular}


Miller 1997 (Continued)

Selective reporting (re- Unclear risk Study not registered. All expected outcomes from methods section reported. porting bias)

Morton 2018

\section{Study characteristics}

\begin{tabular}{|c|c|}
\hline Methods & Randmonised, multinational, multi-centre, non-inferiority RCT. PP analysis. \\
\hline \multirow[t]{5}{*}{ Participants } & Germany, UK. \\
\hline & $\begin{array}{l}\text { Inclusion criteria: One to three non-aggressive } \mathrm{BCC} \text { with less than or equal to } 2 \mathrm{~mm} \text { thickness, } 0.5 \mathrm{~cm} \text { to } \\
2 \mathrm{~cm} \text { diameter, on face/scalp, neck/trunk or extremities. }\end{array}$ \\
\hline & $\begin{array}{l}\text { Exclusion criteria: patients with porphyria, photodermatoses, intolerances to ingredients, recent treat- } \\
\text { ments potentially influencing the ocutome. }\end{array}$ \\
\hline & Age: 66.5 in MAL group (+/- 11.5 SD), 67.3 in ALA group (+/- 11.6 SD). \\
\hline & Gender: male $=131$, female $=100$ \\
\hline \multirow[t]{5}{*}{ Interventions } & Sample size: 281 participants \\
\hline & Period of inclusion: January 2014 to November 2015 \\
\hline & $\begin{array}{l}\text { One obligatory PDT cycle with } 2 \text { PDT sessions } 1 \text { week apart, and a second PDT cycle in case of partial or } \\
\text { no response } 12 \text { weeks after the first cycle: }\end{array}$ \\
\hline & T1: BF-200 ALA PDT $(n=121)$ \\
\hline & T2: MAL PDT $(n=110)$ \\
\hline \multirow[t]{2}{*}{ Outcomes } & Primary outcome measure: overall complete clinical repose of all lesions in each patient at 12 weeks \\
\hline & $\begin{array}{l}\text { Secondary outcome measures: clinical clearance rate of individual lesions at weeks } 4 \text { and } 12 \text {. Cosmetic } \\
\text { outcome as rated by patient satisfaction on a four-point scale. Patient-reported local adverse reactions } \\
\text { as rated on a three-point scale, pain as rated on a ten-point scale and adverse events on a four-point } \\
\text { scale. }\end{array}$ \\
\hline \multirow[t]{2}{*}{ Notes } & Supported by Biofrontera. \\
\hline & Multiple conflicts of interest declared. \\
\hline
\end{tabular}

\section{Risk of bias}

\begin{tabular}{lll}
\hline Bias & Authors' judgement & Support for judgement \\
\hline $\begin{array}{l}\text { Random sequence genera- } \\
\text { tion (selection bias) }\end{array}$ & Low risk & $\begin{array}{l}\text { Quote: "a validated program that automates the random assignment of treat- } \\
\text { ments to randomizations numbers." }\end{array}$ \\
\hline $\begin{array}{l}\text { Allocation concealment } \\
\text { (selection bias) }\end{array}$ & Low risk & $\begin{array}{l}\text { Randomization schedule was generated by Accovion } \\
\text { GmbH (Eschborn, Germany). Specific details not given but likely to have been } \\
\text { concealed adequately. }\end{array}$ \\
\hline
\end{tabular}

Blinding of participants Unclear risk Participants not blinded but unclear if this would influence study outcomes.
and personnel (perfor-

mance bias)

All outcomes 
Morton 2018 (Continued)

Blinding of outcome as- Low risk Observer-blinded. Quote: "To guarantee the blind status of investigators assessment (detection bias) sessing efficacy and safety, a second investigator or delegated person was to All outcomes perform the PDT treatment and all safety evaluations at all visits where PDT was performed."

Incomplete outcome data Low risk Numbers lost to follow-up relatively small and balanced between groups.
(attrition bias)

All outcomes

Selective reporting (re- Low risk porting bias)

\section{Study characteristics}

\begin{tabular}{ll}
\hline Methods & Single-centre, parallel group RCT. ITT analysis. \\
\hline Participants & The Netherlands. \\
& Inclusion criteria: adults with previously untreated HP nBCC with a largest diameter of 20 mm \\
& $\begin{array}{l}\text { Exclusion criteria: pregnancy, life expectancy of }<5 \text { years, known skin cancer syndromes, phototox- } \\
\text { ic/photosensitive drugs, hypersensitivity to light or ALA cream, recurrent or pigmented BCC, histologi- } \\
\text { cal subtypes other than nodular BCC, localisation on concave areas or hairy skin. }\end{array}$ \\
& Age: mean 64.7 years $+/-13$ \\
& Gender: male $=75$, female $=74$
\end{tabular}

Sample size: 149 participants with 173 BCC
Period of inclusion: August 2002 and February 2006.
3 weeks prior to PDT lesions underwent partial debulking curettage under local anaesthesia:
T1: ALA-PDT ( $n=83)$
T2: SE ( $n=88,3 \mathrm{~mm}$ margin)

\section{Outcomes}

Clinical recurrence at 3,6,12,18 months and 2,3,4, and 5 years (confirmed histologically as no recurrence within $5 \mathrm{~mm}$ of the scar).

Notes No declarations of interest

\section{Risk of bias}

\begin{tabular}{lll}
\hline Bias & Authors' judgement & Support for judgement \\
\hline $\begin{array}{l}\text { Random sequence genera- } \\
\text { tion (selection bias) }\end{array}$ & Low risk & $\begin{array}{l}\text { Randomisation per tumour using a computer-generated random allocation } \\
\text { scheme. }\end{array}$ \\
\hline $\begin{array}{l}\text { Allocation concealment } \\
\text { (selection bias) }\end{array}$ & Low risk & $\begin{array}{l}\text { Quote: "A person who was not involved in the trial performed randomization } \\
\text { per tumour." }\end{array}$ \\
\hline $\begin{array}{l}\text { Blinding of participants } \\
\begin{array}{l}\text { and personnel (perfor- } \\
\text { mance bias) }\end{array}\end{array}$ & Unclear risk & $\begin{array}{l}\text { Patients and treating physicians unblinded but unclear if this would influence } \\
\text { performance. }\end{array}$ \\
\hline
\end{tabular}


Mosterd 2008 (Continued)

All outcomes

\begin{tabular}{|c|c|c|}
\hline $\begin{array}{l}\text { Blinding of outcome as- } \\
\text { sessment (detection bias) } \\
\text { All outcomes }\end{array}$ & Unclear risk & $\begin{array}{l}\text { Quote: "Dermatologists other than the research physician conducted the on- } \\
\text { cological follow-up in most cases." } \\
\text { An "independent investigator" looked at the histopathology. }\end{array}$ \\
\hline $\begin{array}{l}\text { Incomplete outcome data } \\
\text { (attrition bias) } \\
\text { All outcomes }\end{array}$ & Unclear risk & $\begin{array}{l}\text { Missing outcome data presented. Fairly large dropouts over the study- unclear } \\
\text { if related to true outcomes. }\end{array}$ \\
\hline $\begin{array}{l}\text { Selective reporting (re- } \\
\text { porting bias) }\end{array}$ & Unclear risk & Study not registered. All expected outcomes from methods section reported. \\
\hline
\end{tabular}

Selective reporting (re- Unclear risk
porting bias)

(20)

Punjabi 2008

\section{Study characteristics}

\begin{tabular}{|c|c|}
\hline Methods & Multi-centre, parallel, double-blinded RCT. ITT analysis. \\
\hline \multirow[t]{5}{*}{ Participants } & UK. \\
\hline & $\begin{array}{l}\text { Inclusion criteria: adults with HP BCC of any type except morphoeic, measuring at least } 0.5 \mathrm{~cm} \text { diame- } \\
\text { ter. }\end{array}$ \\
\hline & $\begin{array}{l}\text { Exclusion criteria: pregnant or lactating patients, those with known sensitivities, immunosuppression, } \\
\text { recent 5-FU or topical tretinoin treatments, history of recurrent BCC after surgery/cryosurgery/radio- } \\
\text { therapy. }\end{array}$ \\
\hline & Age: Range $32-95$ years. \\
\hline & Gender: male $=50$, female $=44$ \\
\hline \multirow[t]{4}{*}{ Interventions } & Sample size: 94 participants. \\
\hline & Period of inclusion: not stated. \\
\hline & T1: Zycure cream, containing $0.005 \%$ of solasodine glycosides every 12 hours for 8 weeks $(n=62)$ \\
\hline & T2: Vehicle (control) every 12 hours for 8 weeks $(n=32)$ \\
\hline \multirow[t]{2}{*}{ Outcomes } & Primary outcome measure: histologically-confirmed clearance of test lesion BCC at 8 weeks. \\
\hline & $\begin{array}{l}\text { Secondary outcome measures: physician's global evaluation of response to treatment, assessment of } \\
\text { local irritation and cosmetic outcome on a four-point scale, and adverse events at screening and end of } \\
\text { treatment. }\end{array}$ \\
\hline Notes & Sponsored by GlycoMed Sciences Lt \\
\hline
\end{tabular}

\section{Risk of bias}

\begin{tabular}{lll}
\hline Bias & Authors' judgement & Support for judgement \\
\hline $\begin{array}{l}\text { Random sequence genera- } \\
\text { tion (selection bias) }\end{array}$ & Unclear risk & Insufficient information to make judgement. \\
\hline $\begin{array}{l}\text { Allocation concealment } \\
\text { (selection bias) }\end{array}$ & Unclear risk & Insufficient information to make judgement. \\
\hline
\end{tabular}


Punjabi 2008 (Continued)

Blinding of participants Low risk The investigator, pharmacist, and the patients were blinded to treatment. and personnel (performance bias)

All outcomes

\begin{tabular}{lll}
\hline $\begin{array}{l}\text { Blinding of outcome as- } \\
\text { sessment (detection bias) } \\
\text { All outcomes }\end{array}$ & Low risk & See above. \\
\hline $\begin{array}{l}\text { Incomplete outcome data } \\
\text { (attrition bias) }\end{array}$ & High risk & $\begin{array}{l}\text { Large difference in proportion of patients withdrawing between groups. } \\
\text { Quote: "For participants who withdrew and missing data, we carried forward } \\
\text { the last observation." }\end{array}$ \\
\hline $\begin{array}{l}\text { Selective reporting (re- } \\
\text { porting bias) }\end{array}$ & Unclear risk & Study not registered. All expected outcomes from methods section reported. \\
\hline
\end{tabular}

Rhodes 2004

\section{Study characteristics}

\begin{tabular}{|c|c|}
\hline Methods & Multi-centre RCT. PP analysis. \\
\hline \multirow[t]{5}{*}{ Participants } & UK. \\
\hline & Inclusion criteria: previously untreated nodular BCC in adults \\
\hline & $\begin{array}{l}\text { Exclusion criteria: more than } 10 \text { lesions, midface region/orbital/ears, lesions }<6 \mathrm{~mm} \text { or }>15 \mathrm{~mm} \text { on the } \\
\text { face/scalp, }>20 \mathrm{~mm} \text { on limbs/neck or }>30 \mathrm{~mm} \text { on trunk, pigmented/morpheaform BCC, porphyria, Gor- } \\
\text { lin syndrome, arsenic exposure, involved in other study, poor compliance, immunosuppression, preg- } \\
\text { nancy or breastfeeding. }\end{array}$ \\
\hline & Age: Mean 69 in T1 (40-95) and 67 in T2 (38-82) \\
\hline & Gender: male $=61$, female $=40$ \\
\hline
\end{tabular}

\begin{tabular}{ll}
\hline Interventions & Sample size: 103 participants, $105 \mathrm{BCC}$ \\
& Period of inclusion: October 1999 to September 2000 \\
T1: MAL-PDT $\left(\mathrm{n}=52,75 \mathrm{~J} / \mathrm{cm}^{2}\right.$ red light, $570 \mathrm{~nm}$ to $\left.670 \mathrm{~nm}\right)$ \\
T2: SE ( $\mathrm{n}=49$, with $5 \mathrm{~mm}$ margin)
\end{tabular}

Outcomes Primary outcome measure: clinical clearance at 3 months.

Secondary outcome measures: sustained response rate at 12 and 24 months. Patient and assessor-reported cosmetic outcome at 3, 12 and 24 months on a four-point rated scale.

Notes Supported by PhotoCure ASA.

\section{Risk of bias}

\begin{tabular}{lll}
\hline Bias & Authors' judgement & Support for judgement \\
\hline $\begin{array}{l}\text { Random sequence genera- } \\
\text { tion (selection bias) }\end{array}$ & Low risk & Computer randomisation \\
\hline
\end{tabular}


Rhodes 2004 (Continued)

\begin{tabular}{|c|c|c|}
\hline $\begin{array}{l}\text { Allocation concealment } \\
\text { (selection bias) }\end{array}$ & Low risk & $\begin{array}{l}\text { Quote: "The randomization list was kept centrally, and investigators called or } \\
\text { faxed to the monitor when a new patient was included to find out the treat- } \\
\text { ment allocated to that patient." }\end{array}$ \\
\hline
\end{tabular}

\begin{tabular}{|c|c|c|}
\hline $\begin{array}{l}\text { Blinding of participants } \\
\text { and personnel (perfor- } \\
\text { mance bias) }\end{array}$ & Unclear risk & $\begin{array}{l}\text { Unable to blind due to nature of interventions but unclear if this would influ- } \\
\text { ence study outcomes. For cosmetic outcomes however it was felt there would } \\
\text { be a high risk of bias. }\end{array}$ \\
\hline
\end{tabular}

Blinding of outcome as- Unclear risk sessment (detection bias)

All outcomes
Unclear risk Unable to blind due to nature of interventions but unclear if this would influence study outcomes.

Incomplete outcome data Unclear risk Large number of dropouts but unclear if related to study interventions.
(attrition bias)

All outcomes Selective reporting (re- Unclear risk Study not registered. All expected outcomes from methods section reported.
porting bias)

\section{Rogozinski 1997}

\section{Study characteristics}

\begin{tabular}{|c|c|c|}
\hline Methods & \multicolumn{2}{|c|}{ Single-centre, parallel RCT. ITT analysis. } \\
\hline \multirow[t]{5}{*}{ Participants } & \multicolumn{2}{|l|}{ Poland. } \\
\hline & \multicolumn{2}{|c|}{$\begin{array}{l}\text { Inclusion criteria: unclear. } \\
\text { Exclusion criteria: unclear. }\end{array}$} \\
\hline & \multicolumn{2}{|l|}{ Total: 35 participants. } \\
\hline & \multicolumn{2}{|l|}{ Age: unclear. } \\
\hline & \multicolumn{2}{|l|}{ Gender: unclear. } \\
\hline \multirow[t]{3}{*}{ Interventions } & \multicolumn{2}{|c|}{ T1: recombinant IFN-beta } \\
\hline & \multicolumn{2}{|l|}{ T2: placebo } \\
\hline & \multicolumn{2}{|c|}{ T1: 1 MU three times a week. } \\
\hline Outcomes & \multicolumn{2}{|c|}{ Early treatment failure at 16 weeks and recurrence at 2 years. } \\
\hline Notes & \multicolumn{2}{|c|}{ Translated for 2007 review. No declarations of interest. } \\
\hline \multicolumn{3}{|l|}{ Risk of bias } \\
\hline Bias & Authors' judgement & Support for judgement \\
\hline $\begin{array}{l}\text { Random sequence genera- } \\
\text { tion (selection bias) }\end{array}$ & Unclear risk & Insufficient information to make judgement. \\
\hline $\begin{array}{l}\text { Allocation concealment } \\
\text { (selection bias) }\end{array}$ & Unclear risk & Insufficient information to make judgement. \\
\hline
\end{tabular}


Rogozinski 1997 (Continued)

Blinding of participants Unclear risk Insufficient information to make judgement. and personnel (performance bias)

All outcomes

\begin{tabular}{lll}
\hline $\begin{array}{l}\text { Blinding of outcome as- } \\
\text { sessment (detection bias) } \\
\text { All outcomes }\end{array}$ & Unclear risk & Insufficient information to make judgement. \\
\hline $\begin{array}{l}\text { Incomplete outcome data } \\
\text { (attrition bias) }\end{array}$ & Unclear risk & Insufficient information to make judgement. \\
All outcomes & &
\end{tabular}

Selective reporting (re- Unclear risk Study not registered. Insufficient information to make judgement.
porting bias)

\section{Romagosa 2000}

\section{Study characteristics}

\begin{tabular}{ll}
\hline Methods & Single-centre, double-blind parallel-group RCT. ITT analysis. Pilot study. \\
\hline Participants & USA. \\
Inclusion criteria: at least one HP, non-superficial BCC measuring at least $0.7 \mathrm{~cm}$ in diameter, in a \\
healthy individual. \\
Exclusion criteria: systemic disease, women of childbearing age, facial BCCs. \\
Age: not stated \\
Gender: not stated.
\end{tabular}

Interventions

Sample size: 13 participants, 17 BCCs.

Period of inclusion: not stated.

Applied twice daily for 4 consecutive weeks:

$\mathrm{T} 1: 5 \%$ 5-FU in PC vehicle $(\mathrm{n}=$ not stated $)$

T2: $5 \% 5-\mathrm{FU}$ in a petrolatum base $(\mathrm{n}=$ not stated $)$

Outcomes Clinical assessment on four-point scale by blinded investigator, cosmetic appearance, AEs and laboratory assessments at 4, 8,12 and 16 weeks. Histological assessment by blinded assessor at week 16 .

Notes No declarations of interest

\section{Risk of bias}

\begin{tabular}{lll}
\hline Bias & Authors' judgement & Support for judgement \\
\hline $\begin{array}{l}\text { Random sequence genera- } \\
\text { tion (selection bias) }\end{array}$ & Unclear risk & Insufficient information given to make judgement. \\
\hline $\begin{array}{l}\text { Allocation concealment } \\
\text { (selection bias) }\end{array}$ & Unclear risk & Insufficient information given to make judgement. \\
\hline
\end{tabular}


Romagosa 2000 (Continued)

Blinding of participants Low risk Double-blinded. and personnel (performance bias)

All outcomes

\section{Blinding of outcome as- Low risk} sessment (detection bias)

All outcomes
Quote: "lesions were evaluated by a blinded investigator","specimens were evaluated by a dermatopathologist who was blinded with respect to whether the biopsy specimens were from treatment or control groups". Probably done.

Incomplete outcome data Low risk No missing outcome data.
(attrition bias)

(attrition bias)

All outcomes

Selective reporting (re- Unclear risk $\quad$ Study not registered. Insufficient information to make judgement.
porting bias)

\section{Salmanpoor 2012}

\section{Study characteristics}

\begin{tabular}{|c|c|c|}
\hline Methods & \multicolumn{2}{|c|}{ Single-centre, parallel group. ITT analysis. } \\
\hline \multirow[t]{5}{*}{ Participants } & \multirow{2}{*}{\multicolumn{2}{|c|}{$\begin{array}{l}\text { Iran. } \\
\text { Inclusion criteria: HP BCC. }\end{array}$}} \\
\hline & & \\
\hline & \multicolumn{2}{|c|}{ Exclusion criteria: indication for Mohs surgery } \\
\hline & \multicolumn{2}{|c|}{ Age: mean 57.3 years (range 21 - 84 years) } \\
\hline & \multicolumn{2}{|c|}{ Gender: male $=31$, female $=24$} \\
\hline \multirow[t]{5}{*}{ Interventions } & \multicolumn{2}{|c|}{ Sample size: 55 participants, (69 lesions) } \\
\hline & \multicolumn{2}{|c|}{ Period of inclusion: not stated. } \\
\hline & \multicolumn{2}{|c|}{$\mathrm{T} 1: \mathrm{SE}$ ( $\mathrm{n}=24,4 \mathrm{~mm}$ margin) } \\
\hline & \multicolumn{2}{|l|}{ T2: curettage $(n=20)$} \\
\hline & \multicolumn{2}{|c|}{ T3: curettage and cautery $(n=25)$} \\
\hline Outcomes & \multicolumn{2}{|c|}{ Recurrence rate at 28 months } \\
\hline Notes & \multicolumn{2}{|c|}{ No declarations of interest } \\
\hline \multicolumn{3}{|l|}{ Risk of bias } \\
\hline Bias & Authors' judgement & Support for judgement \\
\hline $\begin{array}{l}\text { Random sequence genera- } \\
\text { tion (selection bias) }\end{array}$ & Unclear risk & Quote: "Randomisation" but no further details given. \\
\hline $\begin{array}{l}\text { Allocation concealment } \\
\text { (selection bias) }\end{array}$ & Unclear risk & Insufficient information to make judgement \\
\hline
\end{tabular}


Salmanpoor 2012 (Continued)
Blinding of participants
Unclear risk
Insufficient information to make judgement and personnel (performance bias)

All outcomes

\begin{tabular}{lll}
\hline $\begin{array}{l}\text { Blinding of outcome as- } \\
\text { sessment (detection bias) }\end{array}$ & Unclear risk & Insufficient information to make judgement \\
All outcomes & \\
\hline $\begin{array}{l}\text { Incomplete outcome data } \\
\text { (attrition bias) }\end{array}$ & Unclear risk & Insufficient information to make judgement \\
All outcomes & &
\end{tabular}

\begin{tabular}{l}
$\begin{array}{l}\text { Selective reporting (re- } \\
\text { porting bias) }\end{array}$ \\
\hline
\end{tabular}

Salmivuori 2019

\section{Study characteristics}

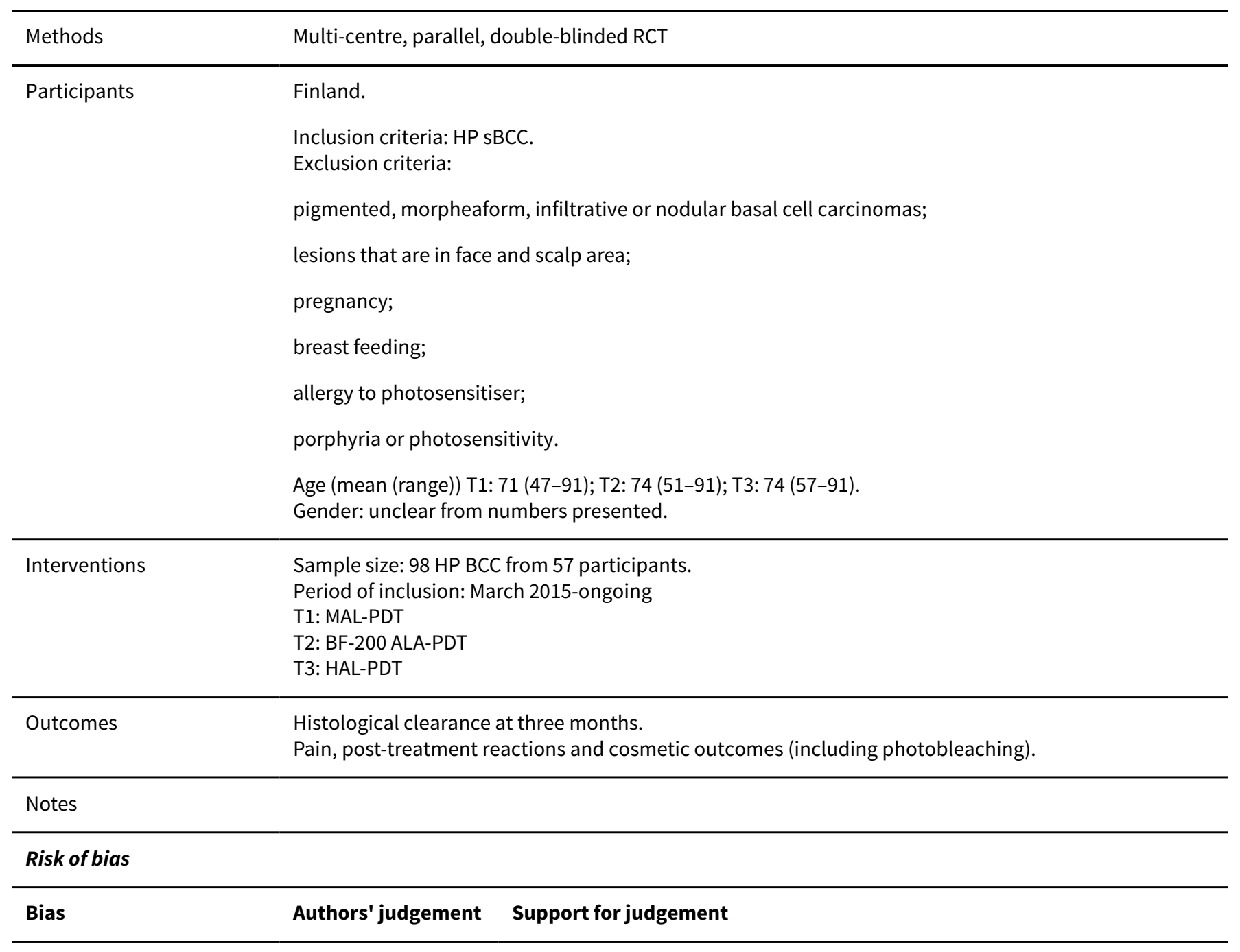


Salmivuori 2019 (Continued)

Random sequence genera- Unclear risk Insufficient information to make judgement. tion (selection bias)

Allocation concealment $\quad$ Unclear risk Insufficient information to make judgement.
(selection bias)

ection bias)

Blinding of participants Unclear risk Insufficient information to make judgement.
and personnel (perfor-
mance bias)
All outcomes

Blinding of outcome as- Unclear risk Insufficient information to make judgement.
sessment (detection bias)

All outcomes

Incomplete outcome data Unclear risk Insufficient information to make judgement.
(attrition bias)

All outcomes

\begin{tabular}{|c|c|c|}
\hline $\begin{array}{l}\text { Selective reporting (re- } \\
\text { porting bias) }\end{array}$ & Unclear risk & $\begin{array}{l}\text { Prospectively registered (NCT02367547). Only abstract with interim results so } \\
\text { unable to assess. }\end{array}$ \\
\hline
\end{tabular}

Schulze 2005

\section{Study characteristics}

\begin{tabular}{ll}
\hline Methods & Multi-centre, phase III, parallel-group, double-blind, vehicle-controlled RCT. ITT analysis. \\
\hline Participants & $\begin{array}{l}\text { Finland, France, Germany, Italy, Spain. } \\
\text { Inclusion criteria: adults with HP sBCC on limbs, trunk, neck or head. Minimum area } 0.5 \mathrm{~cm}^{2} \text { and maxi- } \\
\text { mum diameter } 2 \mathrm{~cm} .\end{array}$ \\
& $\begin{array}{l}\text { Exclusion criteria: clinically significant, unstable medical conditions, pregnancy, metastatic tumours or } \\
\text { high probability thereof, tumours located within } 1 \mathrm{~cm} \text { of the hairline, nose, mouth, ears and eyes, with } \\
\text { no evidence of morphoeic, metaplastic, infiltrative or desmoplastic features, participants with derma- } \\
\text { tological disease in target tumour site. } \\
\text { Age (median): } \mathrm{T} 1=67, \mathrm{~T} 2=68 \\
\text { Gender: male }=101, \text { female }=65\end{array}$ \\
\hline
\end{tabular}

Interventions
Sample size: 166 participants
Period of inclusion: not stated.
Once daily application for six weeks:
T1: imiquimod 5\% $(n=84)$
T2: vehicle $(n=82)$

Outcomes

Clinical clearance at 12 weeks post-treatment, confirmed by blinded histopathological assessment. Investigator-reported skin quality assessment at week 12 post-treatment.

Adverse events at weeks 2, 4 and 6 and post-treatment weeks 4 and 12 .

LSR at all post-treatment visits, assessed according to four-point scale.

Notes Supported by $3 \mathrm{M}$.


Schulze 2005 (Continued)

Multiple authors received funding from or were employed by 3M.

\section{Risk of bias}

\begin{tabular}{|c|c|c|}
\hline Bias & Authors' judgement & Support for judgement \\
\hline $\begin{array}{l}\text { Random sequence genera- } \\
\text { tion (selection bias) }\end{array}$ & Low risk & Quote: "computer generated randomisation schedule" \\
\hline $\begin{array}{l}\text { Allocation concealment } \\
\text { (selection bias) }\end{array}$ & Low risk & $\begin{array}{l}\text { Quote: "Subjects, study personnel and the sponsor's clinical research team } \\
\text { were blinded to study cream identity and treatment assignment." }\end{array}$ \\
\hline $\begin{array}{l}\text { Blinding of participants } \\
\text { and personnel (perfor- } \\
\text { mance bias) } \\
\text { All outcomes }\end{array}$ & Low risk & $\begin{array}{l}\text { Blinding of participants and key study personnel ensured, and unlikely that } \\
\text { the blinding could have been broken. }\end{array}$ \\
\hline $\begin{array}{l}\text { Blinding of outcome as- } \\
\text { sessment (detection bias) } \\
\text { All outcomes }\end{array}$ & Low risk & Blinded investigators including dermatopathologists. \\
\hline $\begin{array}{l}\text { Incomplete outcome data } \\
\text { (attrition bias) } \\
\text { All outcomes }\end{array}$ & Low risk & $\begin{array}{l}\text { Missing outcome data balanced in numbers across intervention groups, with } \\
\text { similar reasons for missing data across groups. }\end{array}$ \\
\hline $\begin{array}{l}\text { Selective reporting (re- } \\
\text { porting bias) }\end{array}$ & Unclear risk & Study not registered. All expected outcomes from methods section reported. \\
\hline
\end{tabular}

\section{Shumack 2002a}

\section{Study characteristics}

\begin{tabular}{|c|c|}
\hline Methods & $\begin{array}{l}\text { Mult-icentre, open-label, dose-response RCT. ITT analysis. First of two studies included in the paper } \\
\text { (see Shumack } 2002 \mathrm{~b} \text { for } 12 \text {-week study). }\end{array}$ \\
\hline Participants & $\begin{array}{l}\text { Australia and New Zealand } \\
\text { Inclusion criteria: adult with single, primary, } \mathrm{HP}, \mathrm{nBCC} \text { (measuring } 0.5 \mathrm{~cm}^{2} \text { to } 1.5 \mathrm{~cm}^{2} \text { ), greater than } 1 \\
\mathrm{~cm} \text { from eyes, nose, mouth, ear and hairline. } \\
\text { Exclusion criteria: } \mathrm{BCC} \text { with morphoeic infiltrating and micronodular patterns. } \\
\text { Age (mean in years): } \mathrm{T} 1=72, \mathrm{~T} 2=66, \mathrm{~T} 3=69, \mathrm{~T} 4=63 \\
\text { Gender: } \text { male }=81 \text {, female }=18\end{array}$ \\
\hline Interventions & $\begin{array}{l}\text { Sample size: } 99 \text { participants } \\
\text { Period of inclusion: Not stated } \\
\text { Topical } 5 \% \text { imiquimod applied for six weeks: } \\
\text { T1: twice daily, seven days per week }(n=1) \\
\text { T2: once daily, seven days per week }(n=35) \\
\text { T3: twice daily, three alternating days per week }(n=31) \\
\text { T4: once daily, three alternating days per week }(n=32)\end{array}$ \\
\hline
\end{tabular}


Shumack 2002a (Continued)

Outcomes Clinical and histological clearance at six weeks post-treatment.

Safety and efficacy evaluations at 1, 2, 4 and 6 weeks.

Local skin reactions according to four-point scale at all visits.

Notes Supported by $3 \mathrm{M}$.

\section{Risk of bias}

\begin{tabular}{|c|c|c|}
\hline Bias & Authors' judgement & Support for judgement \\
\hline $\begin{array}{l}\text { Random sequence genera- } \\
\text { tion (selection bias) }\end{array}$ & Unclear risk & Insufficient information to make judgement. \\
\hline $\begin{array}{l}\text { Allocation concealment } \\
\text { (selection bias) }\end{array}$ & Unclear risk & Insufficient information to make judgement. \\
\hline $\begin{array}{l}\text { Blinding of participants } \\
\text { and personnel (perfor- } \\
\text { mance bias) } \\
\text { All outcomes }\end{array}$ & Unclear risk & Open-label but unclear if this would influence study outcomes. \\
\hline $\begin{array}{l}\text { Blinding of outcome as- } \\
\text { sessment (detection bias) } \\
\text { All outcomes }\end{array}$ & Unclear risk & Open-label but unclear if this would influence study outcomes. \\
\hline $\begin{array}{l}\text { Incomplete outcome data } \\
\text { (attrition bias) } \\
\text { All outcomes }\end{array}$ & Unclear risk & 15/92 discontinued study but unclear in which treatment arms. \\
\hline $\begin{array}{l}\text { Selective reporting (re- } \\
\text { porting bias) }\end{array}$ & Unclear risk & Study not registered. All expected outcomes from methods section reported. \\
\hline
\end{tabular}

Shumack 2002b

\section{Study characteristics}

Multi-centre, open-label, dose-response RCT. ITT analysis. Second of two studies included in the paper
(see Shumack 2002a for 6-week study).

\begin{tabular}{ll}
\hline Participants & USA. \\
Inclusion criteria: adult with single, primary, $\mathrm{HP}, \mathrm{nBCC}$ (measuring $0.5 \mathrm{~cm} 2$ to $1.5 \mathrm{~cm} 2)$, greater than 1 \\
cm from eyes, nose, mouth, ear and hairline. \\
Exclusion criteria: $\mathrm{BCC}$ with morphoeic infiltrating and micronodular patterns. \\
Age (mean in years): $\mathrm{T} 1=53, \mathrm{~T} 2=63, \mathrm{~T} 3=63, \mathrm{~T} 4=58, \mathrm{~T} 5=62$ \\
Gender: male $=63$, female $=29$
\end{tabular}


Shumack 2002b (Continued)

T2: imiquimod once daily, seven days per week $(n=21)$

T3: imiquimod once daily for 5 consecutive days per week $(n=23)$

T4: imiquimod once daily for 3 alternating days per week $(n=20)$

T5: Vehicle $(n=24)$

Outcomes Clinical and histological clearance at six weeks post-treatment.

Safety and efficacy evaluations at 1, 2, 4, 6, 8, 10 and 12 weeks.

Local skin reactions according to four-point scale at all visits.

Notes Supported by $3 \mathrm{M}$.

\section{Risk of bias}

\begin{tabular}{|c|c|c|}
\hline Bias & Authors' judgement & Support for judgement \\
\hline $\begin{array}{l}\text { Random sequence genera- } \\
\text { tion (selection bias) }\end{array}$ & Unclear risk & Insufficient information to make judgement. \\
\hline $\begin{array}{l}\text { Allocation concealment } \\
\text { (selection bias) }\end{array}$ & Unclear risk & Insufficient information to make judgement. \\
\hline $\begin{array}{l}\text { Blinding of participants } \\
\text { and personnel (perfor- } \\
\text { mance bias) } \\
\text { All outcomes }\end{array}$ & Unclear risk & Open-label but unclear if this would influence study outcomes. \\
\hline $\begin{array}{l}\text { Blinding of outcome as- } \\
\text { sessment (detection bias) } \\
\text { All outcomes }\end{array}$ & Unclear risk & Open-label but unclear if this would influence study outcomes. \\
\hline $\begin{array}{l}\text { Incomplete outcome data } \\
\text { (attrition bias) } \\
\text { All outcomes }\end{array}$ & Low risk & Only 3 patients discontinued study. \\
\hline $\begin{array}{l}\text { Selective reporting (re- } \\
\text { porting bias) }\end{array}$ & Unclear risk & Study not registered. All expected outcomes from methods section reported. \\
\hline
\end{tabular}

Siller 2010

\section{Study characteristics}

\begin{tabular}{ll}
\hline Methods & Multi-entre RCT. Modified ITT analysis. \\
\hline Participants & Australia. \\
& Inclusion criteria: adults with HP sBCC, $4 \mathrm{~mm}$ to $15 \mathrm{~mm}$ diameter, maximum thickness $4 \mathrm{~mm}$. \\
& Exclusion criteria: recurrent or atypical lesions, immunosuppression, and prior, concomitant or antici- \\
pated therapy with the potential to confound the study results. women of childbearing potential were \\
also excluded. \\
Mean age years 59 (34 to 86$)$
\end{tabular}


Siller 2010 (Continued)

Gender: male $=88$, female $=32$

Sample size: 60 participants
Period of inclusion: April to December 2005
Topical application by investigator using micro-pipette
T1: ingenol mebutate gel $0.0025 \%(n=16)$
T2: ingenol mebutate gel $0.01 \%(n=16)$
T3: ingenol mebutate gel $0.05 \%(n=16)$
T4: identical vehicle $(n=12)$

Outcomes Clinical response (according to a six-point scale), and histological response at day 85. Local skin reactions on a three-point scale and with descriptive analysis.

Notes Supported by Peplin Ltd.

\section{Risk of bias}

\begin{tabular}{|c|c|c|}
\hline Bias & Authors' judgement & Support for judgement \\
\hline $\begin{array}{l}\text { Random sequence genera- } \\
\text { tion (selection bias) }\end{array}$ & Low risk & $\begin{array}{l}\text { An independent clinical research organisation used a computer-generated } \\
\text { randomisation schedule with a block size of eight. Participants assigned to } \\
\text { treatment or vehicle in a ratio of } 1: 2\end{array}$ \\
\hline $\begin{array}{l}\text { Allocation concealment } \\
\text { (selection bias) }\end{array}$ & Low risk & See above. \\
\hline $\begin{array}{l}\text { Blinding of participants } \\
\text { and personnel (perfor- } \\
\text { mance bias) } \\
\text { All outcomes }\end{array}$ & Unclear risk & $\begin{array}{l}\text { Both the investigator and the participants were blinded. The authors state that } \\
\text { complete blinding could not be guaranteed because of the skin responses ex- } \\
\text { pected with active treatment. The active and vehicle gels were physically in- } \\
\text { distinguishable, and identical packaging used to maintain blinding. }\end{array}$ \\
\hline $\begin{array}{l}\text { Blinding of outcome as- } \\
\text { sessment (detection bias) } \\
\text { All outcomes }\end{array}$ & Low risk & $\begin{array}{l}\text { The post-treatment excision specimen at day } 85 \text { looked at by central patholo- } \\
\text { gist blinded to treatment allocation. }\end{array}$ \\
\hline $\begin{array}{l}\text { Incomplete outcome data } \\
\text { (attrition bias) } \\
\text { All outcomes }\end{array}$ & Low risk & All 60 patients completed follow-up. \\
\hline $\begin{array}{l}\text { Selective reporting (re- } \\
\text { porting bias) }\end{array}$ & Unclear risk & $\begin{array}{l}\text { Study retrospectively registered. All expected outcomes from methods section } \\
\text { reported. }\end{array}$ \\
\hline
\end{tabular}

Smeets 2004

\section{Study characteristics}

\begin{tabular}{ll}
\hline Methods & Multi-centre, unblinded, parallel-group RCT. ITT analysis. \\
\hline Participants & The Netherlands. \\
& $\begin{array}{l}\text { Inclusion criteria: HP primary or recurrent BCC located on the face, at least } 1 \mathrm{~cm} \text { diameter, located in } \\
\text { the H-zone or high-risk/aggressive histopathological subtype. }\end{array}$
\end{tabular}


Smeets 2004 (Continued)

Exclusion: life expectancy less than 3 years.

Age: mean 67.7 years (SD 12.65)

Gender: male $(n=224)$, female $(n=150)$

Sample size: primary BCC 374 participants (408 lesions) and recurrent BCCs 191 participants (204 le-
sions).
Period of inclusion: October 1999 to January 2001
T1: MMS ( $n=204)$
T2: SE $(n=204,3 \mathrm{~mm}$ margin)

$\begin{array}{ll}\text { Outcomes } & \text { Recurrence at } 30 \text { months and } 5 \text { years and incomplete excision rate. Cost-effectiveness after } 5 \text { years. } \\ \text { Participant assessment of cosmetic outcome at } 6 \text { and } 18 \text { months, some lesions also retrospectively as- } \\ \text { sessed by investigators and laymen. }\end{array}$
sessed by investigators and laymen.

Notes Supported by the Dutch Fund for Investigative Medicine

\section{Risk of bias}

\begin{tabular}{|c|c|c|}
\hline Bias & Authors' judgement & Support for judgement \\
\hline $\begin{array}{l}\text { Random sequence genera- } \\
\text { tion (selection bias) }\end{array}$ & Low risk & Randomly assigned using computer programme. \\
\hline $\begin{array}{l}\text { Allocation concealment } \\
\text { (selection bias) }\end{array}$ & Low risk & Secretary not involved in trial undertook the randomisation. \\
\hline $\begin{array}{l}\text { Blinding of participants } \\
\text { and personnel (perfor- } \\
\text { mance bias) } \\
\text { All outcomes }\end{array}$ & Unclear risk & Blinding not possible but unclear if this would influence performance. \\
\hline $\begin{array}{l}\text { Blinding of outcome as- } \\
\text { sessment (detection bias) } \\
\text { All outcomes }\end{array}$ & Unclear risk & $\begin{array}{l}\text { Quote: "For practical reasons, the research physician was not blinded to the } \\
\text { treatment, nor was the patients' own dermatologist" but unclear if this would } \\
\text { influence detection. }\end{array}$ \\
\hline $\begin{array}{l}\text { Incomplete outcome data } \\
\text { (attrition bias) } \\
\text { All outcomes }\end{array}$ & Low risk & $\begin{array}{l}\text { Participants lost to follow-up clearly documented and fairly even between } \\
\text { groups. }\end{array}$ \\
\hline $\begin{array}{l}\text { Selective reporting (re- } \\
\text { porting bias) }\end{array}$ & Unclear risk & $\begin{array}{l}\text { Study retrospectively registered (ISRCTN65009900). All expected outcomes re- } \\
\text { ported. }\end{array}$ \\
\hline
\end{tabular}

\section{Soler 2000}

\section{Study characteristics}

\begin{tabular}{ll}
\hline Methods & Single-centre parallel RCT. ITT analysis. \\
\hline Participants & Norway. \\
& $\begin{array}{l}\text { Inclusion criteria: HP BCC, clinical thickness less than } 1 \mathrm{~mm} \text {, diameter less than } 3 \mathrm{~cm}, \text { fewer than six le- } \\
\text { sions per patient }\end{array}$
\end{tabular}


Soler 2000 (Continued)

Exclusion criteria: not stated.

Age (mean, in years): $\mathrm{T} 1=62.4, \mathrm{~T} 2=62$

Gender: male $=39$, female $=44$

Sample size: 83 participants and 245 lesions.
Inclusion period: study dates not reported.
All lesions in both groups were treated with the same drug (topical application of 20\% ALA). Three
hours later the cream was removed and light source applied. Two different light sources used:
T1: laser light ( $\mathrm{n}=41$ participants, 111 lesions, $630 \mathrm{~nm})$
T2: broadband light $(\mathrm{n}=42$ participants, 134 lesions, $570 \mathrm{~nm}$ to $740 \mathrm{~nm})$

Outcomes Clinical response at 3 and 6 months after treatment according to a three-point scale. Adverse events at one week, patient-reported pain during and throughout follow-up. Cosmetic outcome according to a four-point scale.

Notes No declarations of interest

\section{Risk of bias}

\begin{tabular}{|c|c|c|}
\hline Bias & Authors' judgement & Support for judgement \\
\hline $\begin{array}{l}\text { Random sequence genera- } \\
\text { tion (selection bias) }\end{array}$ & Unclear risk & Method of random sequence generation not given. \\
\hline $\begin{array}{l}\text { Allocation concealment } \\
\text { (selection bias) }\end{array}$ & Low risk & $\begin{array}{l}\text { Quote: "The primary investigator was provided with the randomization num- } \\
\text { bers in locked envelopes, one envelope for each patient." Allocation in blocks } \\
\text { of } 4 \text {. Locked envelopes. }\end{array}$ \\
\hline $\begin{array}{l}\text { Blinding of participants } \\
\text { and personnel (perfor- } \\
\text { mance bias) } \\
\text { All outcomes }\end{array}$ & Unclear risk & $\begin{array}{l}\text { Investigator and patients unblinded but unclear if this would influence study } \\
\text { outcomes. }\end{array}$ \\
\hline $\begin{array}{l}\text { Blinding of outcome as- } \\
\text { sessment (detection bias) } \\
\text { All outcomes }\end{array}$ & Unclear risk & $\begin{array}{l}\text { Investigator and patients unblinded but unclear if this would influence study } \\
\text { outcomes. }\end{array}$ \\
\hline $\begin{array}{l}\text { Incomplete outcome data } \\
\text { (attrition bias) } \\
\text { All outcomes }\end{array}$ & Low risk & No missing outcome data for outcomes of interest. \\
\hline $\begin{array}{l}\text { Selective reporting (re- } \\
\text { porting bias) }\end{array}$ & Unclear risk & Study not registered. All expected outcomes from methods section reported. \\
\hline
\end{tabular}

Spelman 2014

\section{Study characteristics}

\begin{tabular}{ll}
\hline Methods & RCT. Conference abstract. \\
\hline Participants & Australia
\end{tabular}


Exclusion criteria:

Age: 60.8 (mean)

Gender: male $=35$; female $=40$

Sample size: 75 participants
Period of inclusion: April 2011- March 2012
Ingenol mebutate $0.05 \%$ gel applied for three consecutive days with:
T1: full occlusive aluminium disk $(n=27)$
T2: semi-occlusive dressing with OpsiteTM $(n=24)$
T3: no occlusion $(n=24)$

Outcomes Complete histological clearance at day 120. AEs and local skin responses.

Notes No declarations of interest

\section{Risk of bias}

\begin{tabular}{|c|c|c|}
\hline Bias & Authors' judgement & Support for judgement \\
\hline $\begin{array}{l}\text { Random sequence genera- } \\
\text { tion (selection bias) }\end{array}$ & Unclear risk & Insufficient information to make judgement. \\
\hline $\begin{array}{l}\text { Allocation concealment } \\
\text { (selection bias) }\end{array}$ & Unclear risk & Insufficient information to make judgement. \\
\hline $\begin{array}{l}\text { Blinding of participants } \\
\text { and personnel (perfor- } \\
\text { mance bias) } \\
\text { All outcomes }\end{array}$ & Unclear risk & Insufficient information to make judgement. \\
\hline $\begin{array}{l}\text { Blinding of outcome as- } \\
\text { sessment (detection bias) } \\
\text { All outcomes }\end{array}$ & Unclear risk & Insufficient information to make judgement. \\
\hline $\begin{array}{l}\text { Incomplete outcome data } \\
\text { (attrition bias) } \\
\text { All outcomes }\end{array}$ & Unclear risk & Insufficient information to make judgement. \\
\hline $\begin{array}{l}\text { Selective reporting (re- } \\
\text { porting bias) }\end{array}$ & Unclear risk & $\begin{array}{l}\text { Study prospectively registered (NCT01325688). All expected outcomes report- } \\
\text { ed. }\end{array}$ \\
\hline
\end{tabular}

\section{Sterry 2002a}

\section{Study characteristics}

\begin{tabular}{ll}
\hline Methods & Multi-centre (two studies), parallel, open-label. ITT analysis. \\
\hline Participants & Two studies in Europe. \\
\end{tabular}


Sterry 2002a (Continued)

Inclusion criteria: adults with HP primary sBCC on limbs, trunk, neck or head, measuring $0.25 \mathrm{~cm}^{2}$ to 1.5 $\mathrm{cm}^{2}$ if $\mathrm{sBCC}$

Exclusion criteria: previous treatment to target tumour, dermatological conditions interfering with local assessments, tumours located on hands and feet, anogenital area. High-risk areas within $1 \mathrm{~cm}$ of hairline, eyes, nose, mouth or ears were excluded if sBCC but included if nBCC.

Age: mean 63 years (+/- $13.8 \mathrm{SD})$

Gender: male $=59$, female $=34$

Interventions
Period of inclusion: not stated
All treated with imiquimod $5 \%$ for 6 weeks:
T1: 3 times per week with occlusion $(n=23)$
T2 3 times per week without occlusion $(n=25)$
T3: twice per week with occlusion $(n=21)$
T4: twice per week without occlusion $(n=24)$

Outcomes Primary outcome measure: histological confirmation of response at 6 weeks.

Secondary outcome measures: safety and efficacy at 1, 2, 4, 6 and 6 weeks post-treatment. AEs rated on four-point scale Clinical evaluation at 6 weeks.

Notes $\quad$ Funded by $3 \mathrm{M}$.

\section{Risk of bias}

\begin{tabular}{lll}
\hline Bias & Authors' judgement & Support for judgement \\
\hline $\begin{array}{l}\text { Random sequence genera- } \\
\text { tion (selection bias) }\end{array}$ & Low risk & $\begin{array}{l}\text { A computer-generated randomisation schedule assigned patients in blocks of } \\
\text { four to each dosing regimen. }\end{array}$ \\
\hline $\begin{array}{l}\text { Allocation concealment } \\
\text { (selection bias) }\end{array}$ & Unclear risk & $\begin{array}{l}\text { Quote: "Study personnel were blinded to this randomization schedule until af- } \\
\text { ter subject treatment assignments had been made". }\end{array}$ \\
\hline $\begin{array}{l}\text { Blinding of participants } \\
\text { and personnel (perfor- } \\
\text { mance bias) }\end{array}$ & Unclear risk & $\begin{array}{l}\text { Participants and personnel not blinded but unclear if this would influence } \\
\text { study outcomes. }\end{array}$ \\
\hline $\begin{array}{l}\text { Blinding of outcome as- } \\
\text { sessment (detection bias) } \\
\text { All outcomes }\end{array}$ & Unclear risk & Does not state that the dermatopathologists were blinded. \\
\hline $\begin{array}{l}\text { Incomplete outcome data } \\
\begin{array}{l}\text { (attrition bias) } \\
\text { All outcomes }\end{array}\end{array}$ & Low risk & Reasons for withdrawals detailed and balanced between groups. \\
\hline $\begin{array}{l}\text { Selective reporting (re- } \\
\text { porting bias) }\end{array}$ & Unclear risk & Study not registered. All expected outcomes from methods section reported. \\
\hline
\end{tabular}


Sterry 2002b

\section{Study characteristics}

\begin{tabular}{ll}
\hline Methods & Multi-centre (two studies), parallel, open-label. ITT analysis. \\
\hline Participants & Two studies in Europe. \\
Inclusion criteria: adults with HP primary nBCC on limbs, trunk, neck or head, measuring $0.5 \mathrm{~cm} 2$ to 2 \\
$\mathrm{~cm}^{2}$ \\
Exclusion criteria: previous treatment to target tumour, dermatological conditions interfering with lo- \\
cal assessments, tumours located on hands and feet, anogenital area. High-risk areas within $1 \mathrm{~cm}$ of \\
hairline, eyes, nose, mouth or ears were excluded if sBCC but included if nBCC. \\
Age: mean 63 years (+/- 13.8 SD) \\
Gender: male = 59, female $=34$ \\
Sample size: 90 with nBCC \\
Period of inclusion: not stated \\
All treated with imiquimod $5 \%$ for 6 weeks: \\
T1: 3 times per week with occlusion $(\mathrm{n}=23)$ \\
T2 3 times per week without occlusion $(\mathrm{n}=24)$ \\
T3: twice per week with occlusion $(\mathrm{n}=22)$ \\
T4: twice per week without occlusion $(\mathrm{n}=21)$
\end{tabular}

Primary outcome measure: histological confirmation of response at 6 weeks.
Secondary outcome measures: Safety and efficacy at 1, 2, 4, 6 and 6 weeks post-treatment. AEs rated
on four-point scale Clinical evaluation at 6 weeks.

Notes Funded by 3M.

\section{Risk of bias}

\begin{tabular}{lll}
\hline Bias & Authors' judgement & Support for judgement \\
\hline $\begin{array}{l}\text { Random sequence genera- } \\
\text { tion (selection bias) }\end{array}$ & Low risk & $\begin{array}{l}\text { A computer-generated randomisation schedule assigned patients in blocks of } \\
\text { four to each dosing regimen. }\end{array}$ \\
\hline $\begin{array}{l}\text { Allocation concealment } \\
\text { (selection bias) }\end{array}$ & Unclear risk & $\begin{array}{l}\text { Quote: "Study personnel were blinded to this randomization schedule until af- } \\
\text { ter subject treatment assignments had been made". }\end{array}$ \\
\hline $\begin{array}{l}\text { Blinding of participants } \\
\text { and personnel (perfor- } \\
\text { mance bias) }\end{array}$ & Unclear risk & $\begin{array}{l}\text { Participants and personnel not blinded but unclear if this would influence } \\
\text { study outcomes. }\end{array}$ \\
\hline
\end{tabular}

\begin{tabular}{|c|c|c|}
\hline $\begin{array}{l}\text { Blinding of outcome as- } \\
\text { sessment (detection bias) } \\
\text { All outcomes }\end{array}$ & Unclear risk & Does not state that the dermatopathologists were blinded. \\
\hline
\end{tabular}

Incomplete outcome data Low risk $\quad$ Reasons for withdrawals detailed and balanced between groups.
(attrition bias)

(attrition bias)
All outcomes 
Sterry 2002b (Continued)

Selective reporting (reporting bias)

\section{Study characteristics}

\begin{tabular}{ll}
\hline Methods & Multi-centre, parallel-group RCT. PP analysis. \\
\hline Participants & UK, Germany, Switzerland and Australia \\
& Inclusion criteria: adults with HP sBCC, suitable for excision, with no evidence of aggressive growth pat- \\
terns. & \\
& Exclusion criteria: more than 5 lesions, lesions in mid-face region, lesions with a largest diameter < \\
& 8 mm or $>20$ mm, recurrent lesions, lesions in areas of severe sun damage, lesions proximal to cur- \\
rent/previous SCC, pigmented/morphoeaform/infiltrating lesions, pregnancy or breastfeeding, con- \\
traindications to MAL-PDT. \\
Age: mean 63.8 years (range 31 to 92) \\
Gender: male $=194$, female $=132$
\end{tabular}

Interventions

Sample size: 196 participants, 246 sBCC(1.4 lesions per participant).

Period of investigation: not stated.

T1: MAL-PDT $(n=100)$

T2: $\mathrm{SE}(\mathrm{n}=96)$

Outcomes Primary outcome measures: Clinical response at 3 months and investigator-assessed cosmetic out-
come according to a four-point scale at 12 months.

Secondary outcome measures: Clinical response at 12 months, investigator-assessed cosmetic outcome according to a four-point scale at 3, 6 and 12 months.

Other outcomes: AEs at each visit.

Notes Funded by Galderma R\&D, Sophia Antipolis, France.

The investigating authors received payments for the project, and two are Galderma employees.

\section{Risk of bias}

\begin{tabular}{lll}
\hline Bias & Authors' judgement & Support for judgement \\
\hline $\begin{array}{l}\text { Random sequence genera- } \\
\text { tion (selection bias) }\end{array}$ & Low risk & $\begin{array}{l}\text { A block of numbered randomisation cards were provided to each site. Whenev- } \\
\text { er a patient was considered eligible, the investigator had to scratch a randomi- } \\
\text { sation card, following an ascending number order, to disclose the procedure } \\
\text { assigned to the patient. }\end{array}$ \\
\hline $\begin{array}{l}\text { Allocation concealment } \\
\text { (selection bias) }\end{array}$ & Low risk & see above. \\
\hline $\begin{array}{l}\text { Blinding of participants } \\
\text { and personnel (perfor- } \\
\text { mance bias) }\end{array}$ & Unclear risk & $\begin{array}{l}\text { Unable to blind due to nature of treatments but unclear if this would influence } \\
\text { performance. }\end{array}$
\end{tabular}


Szeimies 2008 (Continued)

All outcomes

\begin{tabular}{lll}
\hline $\begin{array}{l}\text { Blinding of outcome as- } \\
\text { sessment (detection bias) }\end{array}$ & Unclear risk & $\begin{array}{l}\text { Unable to blind due to nature of treatments but unclear if this would influence } \\
\text { detection. For cosmetic outcomes however it was felt there would be a high- } \\
\text { All outcomes }\end{array}$
\end{tabular}

All outcomes

Reasons for missing outcome unlikely to be related to true outcome

Incomplete outcome data Low risk

(attrition bias)

All outcomes

Selective reporting (re- Unclear risk porting bias)

Study not registered. All expected outcomes from methods section reported.

Tran 2012

\section{Study characteristics}

\begin{tabular}{ll}
\hline Methods & Single-centre, parallel, pilot RCT. \\
\hline Participants & USA. \\
& Inclusion criteria: HP superficial, nodular, multicentric BCC measuring $0.4 \mathrm{~cm}$ to $3 \mathrm{~cm}$ on trunk and ex- \\
& tremities in Caucasian patients with Fitzpatrick skin types I and II. \\
& Exclusion criteria: morphoeaform, infiltrating and recurrent BCCs or those on the head and neck, feet \\
& or genitals. \\
& Age: range 46 to 84 years, median age 62 (SD $+/-12$ years). \\
& Gender: Male $=10$, female $=10$. \\
\hline
\end{tabular}

Interventions Sample size: 17 participants with $20 \mathrm{HP} \mathrm{BCC.}$

Period of inclusion: not stated.

T1: PDL $7 \mathrm{~mm}$ spot-size, single pulses, $15 \mathrm{~J} / \mathrm{cm} 2(\mathrm{n}=8)$.

T2: PDL $10 \mathrm{~mm}$ spot-size, stacked pulses, $7.5 \mathrm{~J} / \mathrm{cm} 2(\mathrm{n}=8)$.

T3: Control group (no treatment) $(n=7)$.

Outcomes Histological clearance and adverse events at 0 to 157 days.

Notes No declarations of interest.

\section{Risk of bias}

\begin{tabular}{lll}
\hline Bias & Authors' judgement & Support for judgement \\
\hline $\begin{array}{l}\text { Random sequence genera- } \\
\text { tion (selection bias) }\end{array}$ & Unclear risk & Insufficient information to make judgement. \\
\hline $\begin{array}{l}\text { Allocation concealment } \\
\text { (selection bias) }\end{array}$ & Unclear risk & Insufficient information to make judgement. \\
\hline $\begin{array}{l}\text { Blinding of participants } \\
\text { and personnel (perfor- } \\
\text { mance bias) } \\
\text { All outcomes }\end{array}$ & Low risk & Participants were blinded to treatment allocation. \\
\hline $\begin{array}{l}\text { Blinding of outcome as- } \\
\text { sessment (detection bias) }\end{array}$ & Low risk & \\
\hline
\end{tabular}


Tran 2012 (Continued)

All outcomes

\begin{tabular}{lll}
\hline $\begin{array}{l}\text { Incomplete outcome data } \\
\text { (attrition bias) } \\
\text { All outcomes }\end{array}$ & No losses to follow-up. \\
\hline $\begin{array}{l}\text { Selective reporting (re- } \\
\text { porting bias) }\end{array}$ & Unclear risk & $\begin{array}{l}\text { Study not prospectively registered. All expected outcomes from the methods } \\
\text { section reported. }\end{array}$ \\
\hline
\end{tabular}

Wang 2001

\section{Study characteristics}

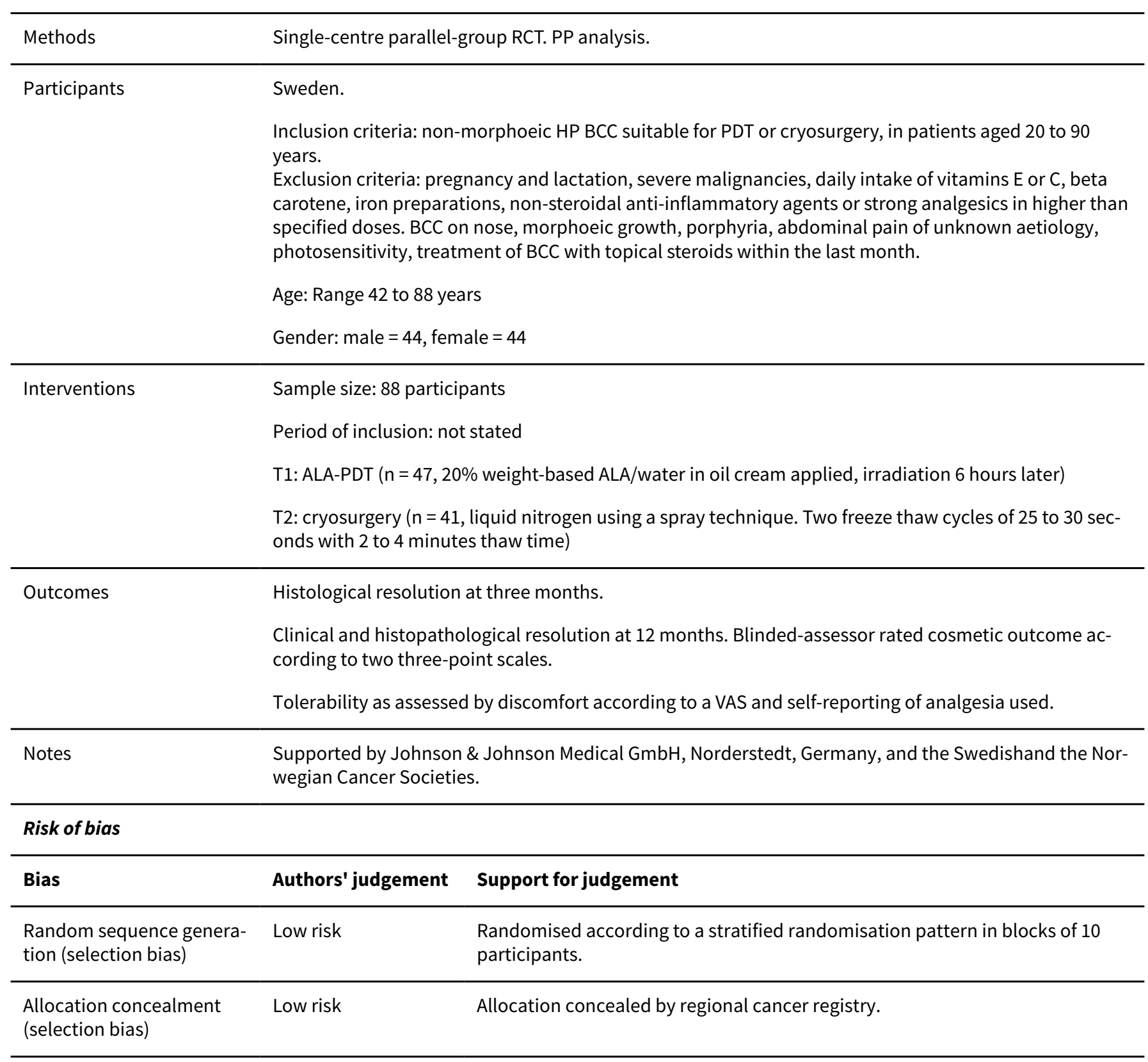


Wang 2001 (Continued)

$\begin{array}{ll}\begin{array}{l}\text { Blinding of participants } \\ \text { and personnel (perfor- }\end{array} & \text { Unclear risk } \\ \text { procedures". Unclear if this would influence study outcomes. }\end{array}$
procedures". Unclear if this would influence study outcomes. mance bias)

All outcomes

\begin{tabular}{|c|c|c|}
\hline $\begin{array}{l}\text { Blinding of outcome as- } \\
\text { sessment (detection bias) } \\
\text { All outcomes }\end{array}$ & Unclear risk & $\begin{array}{l}\text { Quote: "No blinding regimen was possible due to the nature of the treatment } \\
\text { procedures." For cosmetic outcomes however it was felt there would be a high } \\
\text { risk of bias. }\end{array}$ \\
\hline
\end{tabular}

All outcomes risk of bias.

Incomplete outcome data Low risk

No missing outcome data.

(attrition bias)

All outcomes

Selective reporting (re- Unclear risk Study not registered. All expected outcomes from methods section reported.
porting bias)

AEs: advere events;ALA: aminolevulinic acid; BCC: basal cell carcinoma; nBCC: nodular BCC; sBCC: superficial BCC; HP: histologically proven; IFN: interferons; ITT: intention-to-treat; IU: international unit; MAL: methyl aminolevulinate; MMS: Mohs micrographic surgery; NSAIDs: non-steroidal anti-inflammatories; MU: megaunits; PC: phosphatidyl choline;PDL: pulsed dye laser; PDT: photodynamic therapy; PP: per protocol;RCT: randomised controlled trial; SCC: squamous cell carcinoma; SE: surgical excision; T1: treatment group 1; T2: treatment group 2; T3: treatment group 3; 5-FU: 5-fluorouracil; 5-FU/epi: 5-fluorouracil/epinephrine; VAS: visual analogue scale.

Characteristics of excluded studies [ordered by study ID]

\begin{tabular}{|c|c|}
\hline Study & Reason for exclusion \\
\hline Al-Niaimi 2015 & $\begin{array}{l}\text { No outcomes of interest measured - study measured outcomes relating to number of sections and } \\
\text { stages. }\end{array}$ \\
\hline Arits 2014 & $\begin{array}{l}\text { No outcomes of interest measured - study addressed cost-effectiveness. Arits } 2013 \text { study is includ- } \\
\text { ed which measured outcomes of interest. }\end{array}$ \\
\hline Berroeta 2007 & Not clear if biopsy-proven BCC. \\
\hline Butler 2009 & No outcomes of interest measured, study addressed stages and defect size. \\
\hline Čarija 2016 & Not an RCT, intra-patient study. \\
\hline Cho 2013 & Not an RCT. \\
\hline de Haas 2006 & Not all BCCs histologically proven. \\
\hline Dessinioti 2014 & Not an RCT. \\
\hline EudraCT 2013-000092-33 & Not all BCCs histologically proven. \\
\hline Genouw 2018 & Not an RCT, intra-patient randomised study. \\
\hline Groselj 2018 & Not an RCT. \\
\hline Huang 2004 & $\begin{array}{l}\text { No outcomes of interest measured. This study was about the utility of preoperative tumour curet- } \\
\text { tage in Mohs micrographic surgery for primary or recurrent BCC. The end points were per cent sur- } \\
\text { face area increase from tumour surface area to wound surface area; actual surface area increase } \\
\text { from tumour surface area to wound surface area; and number of layers removed to clear tumour. }\end{array}$ \\
\hline
\end{tabular}




\begin{tabular}{|c|c|}
\hline Study & Reason for exclusion \\
\hline Ibbotson 2018 & Not all BCCs histologically proven. \\
\hline Lippert 2013 & Included previously treated lesions. \\
\hline Lu 2017 & Included several tumour types. Data related to BCC alone not available. \\
\hline Lui 2004 & Included Gorlin syndrome patients. \\
\hline Migden 2015 & Included previously treated lesions. \\
\hline NCT01033019 & No outcomes of interest measured. \\
\hline Nguyen 2018 & No outcomes of interest measured. \\
\hline Quirk 2010 & Not an RCT. \\
\hline Radiotis 2014 & Not an RCT. \\
\hline Schleier 2007 & Included participants with Gorlin syndrome. \\
\hline Sekulic 2012 & Not an RCT \\
\hline Spencer 2006 & Adjuvant therapy study. \\
\hline Tang 2012 & Included participants with Gorlin syndrome. \\
\hline van der Geer 2012 & No outcomes of interest measured. \\
\hline Vijlder 2012 & Not all lesions histologically proven. \\
\hline Wettstein 2013 & Not all lesions histologically proven. \\
\hline Zane 2017 & Not all lesions histologically proven. \\
\hline
\end{tabular}

BCC: basal cell carcinoma;RCT: randomised controlled trial.

Characteristics of studies awaiting classification [ordered by study ID]

Bunker 2000

\begin{tabular}{ll}
\hline Methods & Unknown \\
\hline Participants & Unknown \\
\hline Interventions & 5- Fluorouracil/adrenaline injectable gel. \\
\hline Outcomes & Unknown \\
\hline Notes & $\begin{array}{l}\text { This trial was identified from the National Research Register in our old review searches but no more } \\
\text { details of the study have been found and we are uncertain if this is an RCT despite attempts to con- } \\
\text { tact the author. }\end{array}$ \\
\hline
\end{tabular}


IRCT $2017030732933 \mathrm{~N} 1$

\begin{tabular}{ll}
\hline Methods & Single-centre, randomised, parallel RCT. \\
\hline Participants & Iran. \\
& $\begin{array}{l}\text { Inclusion criteria: pathologically documented BCC; maximum tumour diameter of } 30 \text { mm. } \\
\text { Exclusion criteria: skins with Fitzpatrick type of higher than 3; coagulation disorders or recent re- } \\
\text { ceiving of anticoagulant agents; consumption of light-sensitive drugs. }\end{array}$ \\
\hline Interventions & $\begin{array}{l}\text { T1: } 585 \text {-nanometer PDL laser with power of } 9 \text { joules, in } 450 \text { microseconds, for } 2 \text { treatment sessions } \\
\text { with 3-week intervals; } 72 \text { 585-nanometer PDL laser with power of } 9 \text { joules, in } 450 \text { microseconds, } \\
\text { followed by cryosurgery with one unit of liquid nitrogen (CRY-ACW) using spray method during two } \\
\text { freeze cycles each of which being } 4 \pm 2 \text { minutes, for } 2 \text { sessions with intervals of } 3 \text { weeks. }\end{array}$ \\
\hline Outcomes & Primary: tumour remission 6 months after the last treatment session. \\
\hline Notes & Recruitment completed in 2017. Results not published and author not responded to email. \\
\hline
\end{tabular}

\section{ISRCTN 92678315}

\begin{tabular}{ll}
\hline Methods & Single-centre, randomised, parallel RCT. \\
\hline Participants & UK. \\
& $\begin{array}{l}\text { Participant inclusion criteria } \\
\text { 1. Patients referred for PDT as judged appropriate by dermatologists in local area } \\
\text { 2. Patients will have BCCs, Bowen's disease or actinic keratoses and will be randomised depending } \\
\text { on diagnosis. }\end{array}$ \\
\hline Interventions & $\begin{array}{l}\text { Two treatment arms in the study, randomised in double-blind fashion to receive either topical } \\
\text { which the cream has been applied. Comparison of the two groups will be by change in size of skin } \\
\text { lesion by any tolerance of procedure. }\end{array}$ \\
\hline Outcomes & Response of skin lesion clinically by size change at 3-month follow-up. \\
\hline Notes & Recruitment completed in 2003. Results not published and author not responded to email. \\
\hline
\end{tabular}

Kang 2018

\begin{tabular}{ll}
\hline Methods & Single-centre, randomised, parallel RCT - abstract only. \\
\hline Participants & China. \\
& Unclear inclusion criteria \\
& Age: not stated. \\
& Gender: not stated. \\
\hline Interventions & T2 participants. Unclear BCC subtype. Unclear if HP. \\
T1: 635 nm Ella light once a week for 4 weeks. \\
T2: imiquimod pre-treatment and 635 nm Ella light once a week for 4 weeks. \\
\hline
\end{tabular}


Kang 2018 (Continued)

Outcomes Immune index changes, clinical efficacy, cosmetic effect and adverse reactions.

Notes

Chinese study. Only translated English abstract obtained. Unable to access full paper or contact authors.

Ma 2018

\begin{tabular}{ll}
\hline Methods & Randomised, parallel RCT - abstract only. \\
\hline Participants & China. \\
& Unclear inclusion criteria \\
& Age: not stated. \\
& Gender: not stated. \\
\hline
\end{tabular}

Interventions
$\begin{aligned} & 40 \text { participants. } \\ & \text { T1: adriamycin combined with cisplatin. } \\ & \text { T2: cisplatin. }\end{aligned}$

Outcomes

Quote: "Observe and compare the clinical effects, adverse reactions, serum VEGF level, pain score and self-efficacy of two groups."

Notes

Chinese study. Only translated English abstract obtained. Unable to access full paper or contact authors.

\section{RPCEC00000147}

\begin{tabular}{ll}
\hline Methods & Multi-centre, randomised, parallel RCT. \\
\hline Participants & Cuba. \\
& Inclusion criteria \\
1. Clinical and histological diagnosis of BCC & 2. $>18$ years of age. \\
3. Lesions between $1.5 \mathrm{~cm}^{2}$ to $10.0 \mathrm{~cm}^{2}$. \\
4.Lesions of any subtype, localisation and size \\
5. Non recurrent lesion. \\
6. Without previous specific treatments. \\
Study group (CIGB-128-A). Perilesional treatment with CIGB-128-A (dose of 10.5 MIU) 2 times per \\
week for 4 weeks Study group (CIGB-128-A). Perilesional treatment with CIGB-128-A (dose of 10.5 \\
MIU) 2 times per week for 6 weeks Study group (CIGB-128-A). Perilesional treatment with CIGB-128- \\
A (dose of 10.5 MIU) 2 times per week for 8 weeks Control group (CIGB-128-A). Perilesional treat- \\
ment with CIGB-128-A (dose of 10.5 MIU) 3 times per week for 4 weeks Study group (CIGB-128- \\
A). Perilesional treatment with CIGB-128-A (dose of 10.5 MIU) 3 times per week for 6 weeks Study \\
group (CIGB-128-A). Perilesional treatment with CIGB-128-A (dose of 10.5 MIU) 3 times per week for \\
8 weeks.
\end{tabular}

\section{Outcomes}

Primary outcomes 
Clinical response (Size of the lesion. RECIST criteria. Complete Response (CR); Partial Response (PR); Stable Disease (SD) and Progression Disease (PD)). Measuring time: at baseline and at week 16 after starting the treatment.

Secondary outcomes

Clinical response time (time to reach partial or complete response). Measuring time: weeks 1, 4, 8, 12 and 16. Quality of scarring; Will be evaluated since the clinical standpoint, in patients that respond completely to treatment. Measuring time: at a week 16 and annually for 10 years. Time to recurrence (time elapsing from the first day provided the absence of neoplastic cells according to the evaluation histological or clinical (patient refusal to biopsy end or biopsy no useful), until appears an injury histologically demonstrated in the treated site). Measuring time: annually for 10 years. Presence of clinical adverse effects. Anti-IFN alpha and gamma antibodies.

Notes

Date of first enrolment: 01/02/2013. Recruitment status: Pending. No reply to email to trial registration contact.

ALA: aminolevulinic acid; BCC: basal cell carcinoma; EDTA: Ethylenediaminetetraacetic acid; HP: histologically proven; PDL: pulsed dye laser; PDT: photodynamic therapy; RCT: randomised controlled trial; VEGF: Vascular endothelial growth factor.

\section{Characteristics of ongoing studies [ordered by study ID]}

EudraCT 2016-002255-25

\begin{tabular}{ll}
\hline Study name & Electrochemotherapy versus standard radiotherapy for the treatment of basal cell carcinoma. \\
\hline Methods & Single-centre, randomised, parallel RCT. \\
\hline Participants & Denmark. \\
& Age > 18 with HP BCC. \\
& Aiming for 52 participants. \\
\hline Interventions & T1: bleomycin electrochemotherapy. \\
\hline T2: standard radiotherapy (no further detail given). \\
\hline $\begin{array}{l}\text { Primary: evaluate tumour response and compare efficacy of electrochemotherapy versus standard } \\
\text { radiotherapy (time point: 3 months). } \\
\text { Secondary: adverse effects (up to 5 years after treatment). }\end{array}$ \\
\hline Contact information & 2016. \\
\hline
\end{tabular}

\section{Notes}

\section{NCT02242929}

\begin{tabular}{ll}
\hline Study name & $\begin{array}{l}\text { Surgical excision versus combined treatment with curettage and imiquimod for nodular basal cell } \\
\text { carcinoma: an open, non-inferiority, randomized controlled trial }\end{array}$ \\
\hline Methods & Single-centre, randomised, parallel RCT \\
\hline Participants & Netherlands. \\
& Age:18-90.
\end{tabular}


NCT02242929 (Continued)

Primary $\mathrm{HP} \mathrm{nBCC} \geq 4 \mathrm{~mm}$ and $\leq 20 \mathrm{~mm}$ in diameter.

Aiming for 145 participants.

T2: Imiquimod 5\% cream with prior curettage

Outcomes

Primary: proportion of participants tumour-free [Time Frame: At 1 year after end of treatment]

Secondary: 5-year cumulative probability of recurrence-free survival after end of treatment [Time

Frame: At 5 years after end of treatment].

Compliance [Time Frame: At 3 months after end of treatment ].

Cosmetic appearance [ Time Frame: At 1 and 5-year after end of treatment ].

Patient satisfaction [ Time Frame: At 1 and 5-year after end of treatment ].

Level of Pain [ Time Frame: 2 weeks after end of treatment ].

Cost-effectiveness [ Time Frame: At 5-year after end of treatment ].

Adverse events [ Time Frame: Up to 3 months after end of treatment ].

\begin{tabular}{ll}
\hline Starting date & January 2016. \\
\hline Contact information & Principal Investigator: Klara Mosterd, MD, PhD Maastricht University Medical Centre. \\
\hline Notes & Ongoing study, results expected 2022. \\
\hline
\end{tabular}

NCT03573401

Study name

A randomized, double blind, vehicle-controlled multicenter phase III study to evaluate the safety and efficacy of BF-200 ALA (Ameluz ${ }^{\circledR}$ ) and BF-RhodoLED ${ }^{\circledR}$ in the treatment of superficial basal cell carcinoma (SBCC) With photodynamic therapy (PDT).

\begin{tabular}{ll}
\hline Methods & Multi-centre, randomised, parallel RCT. \\
\hline Participants & USA. \\
& Aiming for 186 participants (over 18 years old) across 15 centres.
\end{tabular}

\begin{tabular}{ll}
\hline Interventions & Experimental: BF-200 ALA \\
& Topical application of BF-200 ALA containing 7.8\% 5-ALA (5-aminolevulinic acid).
\end{tabular}

Placebo comparator: vehicle

Topical application of vehicle to BF-200 ALA containing no active ingredient.

\section{Outcomes}

Primary:

Composite clinical and histological response of the participant's Main Target Lesion as assessed 12 weeks after the start of the last PDT cycle that included treatment of the main target lesion. [Time Frame: 12 weeks after the start of the last PDT cycle that included treatment of the main target lesion]

The composite clinical and histological response rate of the participant's main target lesion is the percentage of participants with clinically and histologically cleared main target lesion 12 weeks after the start of the last PDT cycle that included treatment of the main target lesion (Visit 5 or Visit 8).

\begin{tabular}{ll}
\hline Starting date & 2018. \\
\hline Contact information & b.schmitz@biofrontera.com \\
\hline Notes & Study aiming to complete 2024. \\
\hline
\end{tabular}


ALA: aminolevulinic acid; BCC: basal cell carcinoma; nBCC: nodular basal cell carcinoma; HP: histologically proven; PDT: photodynamic therapy; $\mathbf{R C T}$ : randomised controlled trial.

\section{DATA AND ANALYSES}

Comparison 1. Mohs micrographic surgery vs surgical excision

\begin{tabular}{lllll}
\hline Outcome or subgroup title & $\begin{array}{l}\text { No. of } \\
\text { studies }\end{array}$ & $\begin{array}{l}\text { No. of } \\
\text { partici- } \\
\text { pants }\end{array}$ & Statistical method & Effect size \\
\hline 1.1 Recurrence at 3 years (lesions) & 1 & Risk Ratio (M-H, Random, 95\% Cl) & Totals not selected \\
\hline 1.2 Recurrence at 5 years (lesions) & 1 & Risk Ratio (M-H, Random, 95\% Cl) & Totals not selected \\
\hline
\end{tabular}

Analysis 1.1. Comparison 1: Mohs micrographic surgery vs surgical excision, Outcome 1: Recurrence at 3 years (lesions)

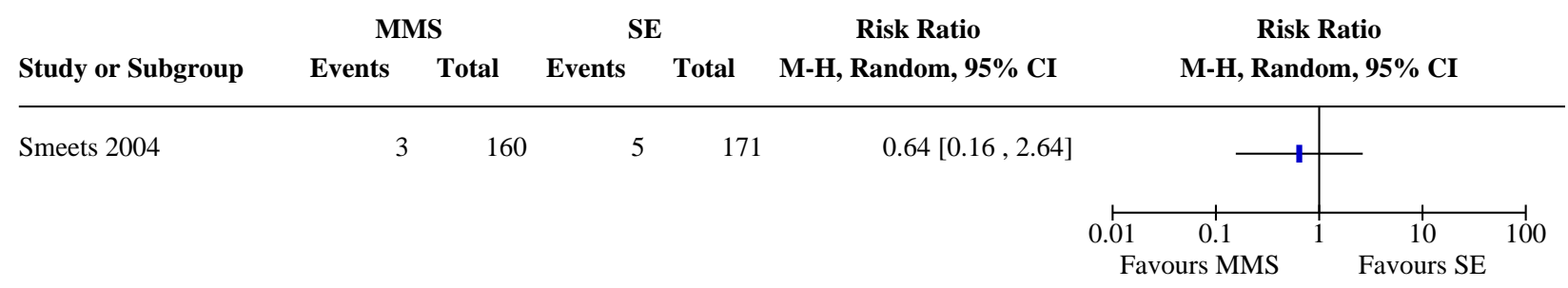

Analysis 1.2. Comparison 1: Mohs micrographic surgery vs surgical excision, Outcome 2: Recurrence at 5 years (lesions)

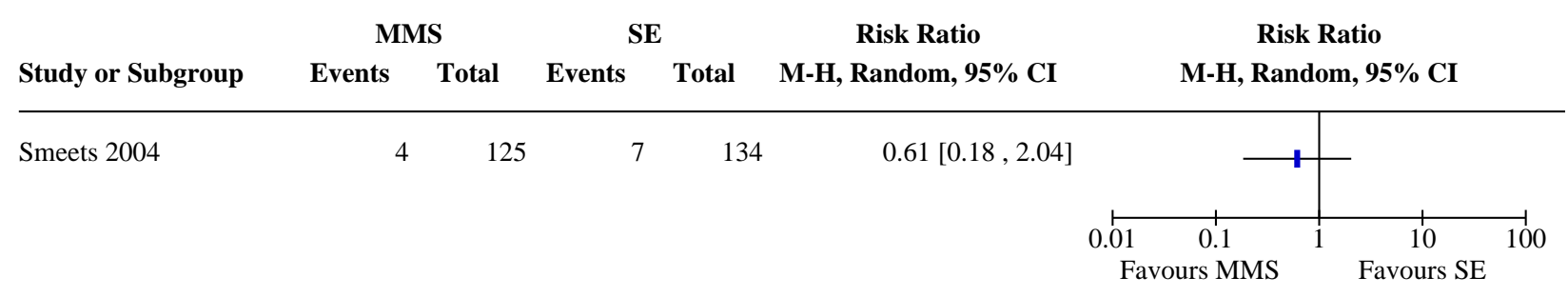

\section{Comparison 2. Imiquimod vs surgical excision}

\begin{tabular}{lllll}
\hline Outcome or subgroup title & $\begin{array}{l}\text { No. of } \\
\text { studies }\end{array}$ & $\begin{array}{l}\text { No. of } \\
\text { partici- } \\
\text { pants }\end{array}$ & Statistical method & Effect size \\
\hline 2.1 Recurrence at 3 years & 1 & & $\begin{array}{l}\text { Risk Ratio (M-H, Random, 95\% } \\
\text { Cl) }\end{array}$ & Totals not selected \\
\hline 2.2 Recurrence at 5 years & 1 & $\begin{array}{l}\text { Risk Ratio (M-H, Random, 95\% } \\
\text { Cl) }\end{array}$ & Totals not selected \\
\hline
\end{tabular}




\begin{tabular}{lllll}
\hline Outcome or subgroup title & $\begin{array}{l}\text { No. of } \\
\text { studies }\end{array}$ & $\begin{array}{l}\text { No. of } \\
\text { partici- } \\
\text { pants }\end{array}$ & Statistical method & Effect size \\
\hline 2.3 Cosmetic outcome (good/excellent) & 1 & & $\begin{array}{l}\text { Risk Ratio (M-H, Random, 95\% } \\
\text { Cl) }\end{array}$ & Totals not selected \\
\hline 2.3.1 Observer rated at 3 years & 1 & $\begin{array}{l}\text { Risk Ratio (M-H, Random, 95\% } \\
\text { Cl) }\end{array}$ & Totals not selected \\
\hline 2.3.2 Participant rated at 3 years & 1 & $\begin{array}{l}\text { Risk Ratio (M-H, Random, 95\% } \\
\text { Cl) }\end{array}$ & Totals not selected \\
\hline 2.4 Pain (moderate/severe) & 1 & $\begin{array}{l}\text { Risk Ratio (M-H, Random, 95\% } \\
\text { Cl) }\end{array}$ & Totals not selected \\
\hline 2.4.1 During treatment & 1 & $\begin{array}{l}\text { Risk Ratio (M-H, Random, 95\% } \\
\text { Cl) }\end{array}$ & Totals not selected \\
\hline 2.4.2 During follow-up & 1 & $\begin{array}{l}\text { Risk Ratio (M-H, Random, 95\% } \\
\text { Cl) }\end{array}$ & Totals not selected
\end{tabular}

\section{Analysis 2.1. Comparison 2: Imiquimod vs surgical excision, Outcome 1: Recurrence at 3 years}

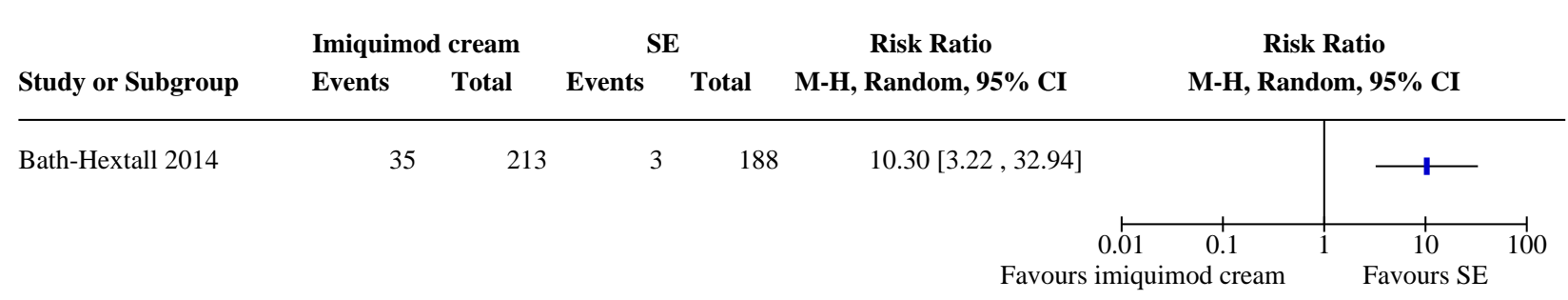

Analysis 2.2. Comparison 2: Imiquimod vs surgical excision, Outcome 2: Recurrence at 5 years

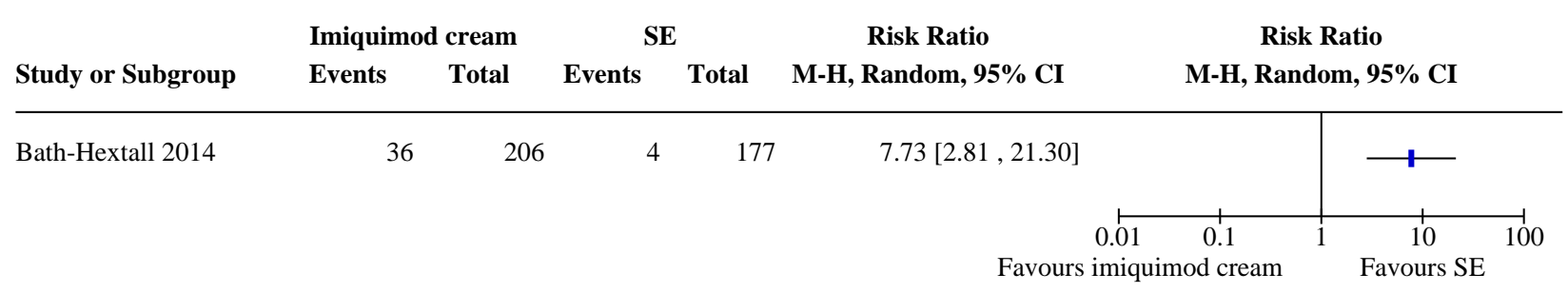


Analysis 2.3. Comparison 2: Imiquimod vs surgical excision, Outcome 3: Cosmetic outcome (good/excellent)

\begin{tabular}{|c|c|c|c|c|c|}
\hline \multirow[b]{2}{*}{ Study or Subgroup } & \multicolumn{2}{|c|}{ Imiquimod cream } & \multicolumn{2}{|c|}{ SE } & \multirow{2}{*}{$\begin{array}{c}\text { Risk Ratio } \\
\text { M-H, Random, 95\% CI }\end{array}$} \\
\hline & Events & Total & Events & Total & \\
\hline \multicolumn{6}{|c|}{ 2.3.1 Observer rated at 3 years } \\
\hline Bath-Hextall 2014 & 103 & 170 & 62 & 174 & $1.70[1.35,2.15]$ \\
\hline \multicolumn{6}{|c|}{ 2.3.2 Participant rated at 3 years } \\
\hline Bath-Hextall 2014 & 147 & 160 & 153 & 166 & $1.00[0.94,1.06]$ \\
\hline
\end{tabular}

2.3.2 Participant rated at 3 years

Bathext

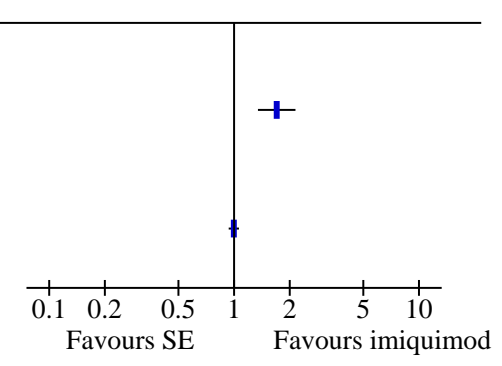

Analysis 2.4. Comparison 2: Imiquimod vs surgical excision, Outcome 4: Pain (moderate/severe)

\begin{tabular}{|c|c|c|c|c|c|c|}
\hline \multirow[b]{2}{*}{ Study or Subgroup } & \multicolumn{2}{|c|}{ Imiquimod cream } & \multicolumn{2}{|c|}{ SE } & Risk Ratio & Risk Ratio \\
\hline & Events & Total & Events & Total & M-H, Random, 95\% CI & M-H, Random, 95\% CI \\
\hline
\end{tabular}

\subsubsection{During treatment}

Bath-Hextall 2014

72

242

$44 \quad 201$

$1.36[0.98,1.88]$

2.4.2 During follow-up

Bath-Hextall 2014

22

233

41

206

$0.47[0.29,0.77]$

M-H, Random, 95\% CI

Comparison 3. Radiotherapy vs surgical excision (with or without frozen section margin control)

\begin{tabular}{|c|c|c|c|c|}
\hline Outcome or subgroup title & $\begin{array}{l}\text { No. of } \\
\text { studies }\end{array}$ & $\begin{array}{l}\text { No. of } \\
\text { partici- } \\
\text { pants }\end{array}$ & Statistical method & Effect size \\
\hline 3.1 Recurrence at 3 years & 1 & & Risk Ratio (M-H, Random, 95\% Cl) & Totals not selected \\
\hline 3.2 Recurrence at 4 years & 1 & & Risk Ratio (M-H, Random, 95\% Cl) & Totals not selected \\
\hline 3.3 Cosmetic outcome (good) & 1 & & Risk Ratio (M-H, Random, 95\% Cl) & Totals not selected \\
\hline 3.3.1 Participant rated at 4 years & 1 & & Risk Ratio (M-H, Random, 95\% Cl) & Totals not selected \\
\hline 3.3.2 Observer rated at 4 years & 1 & & Risk Ratio (M-H, Random, 95\% Cl) & Totals not selected \\
\hline
\end{tabular}


Analysis 3.1. Comparison 3: Radiotherapy vs surgical excision (with or without frozen section margin control), Outcome 1: Recurrence at 3 years

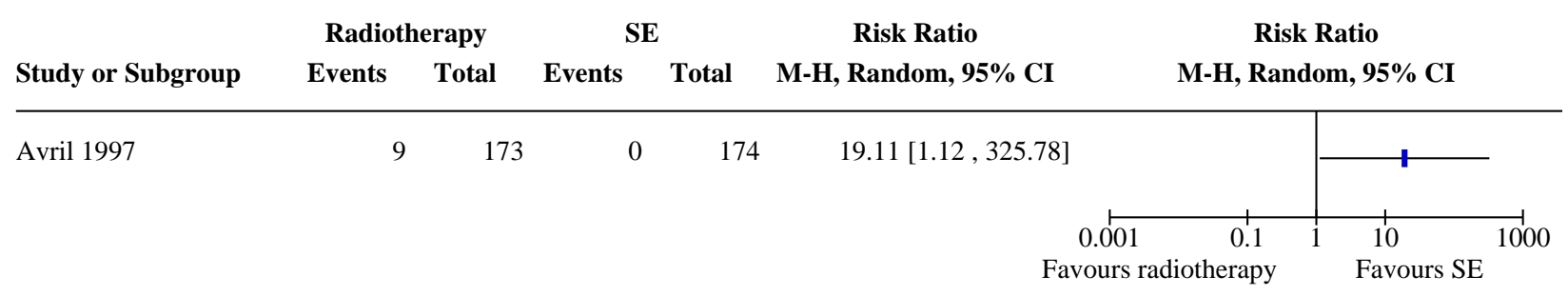

Analysis 3.2. Comparison 3: Radiotherapy vs surgical excision (with or without frozen section margin control), Outcome 2: Recurrence at 4 years

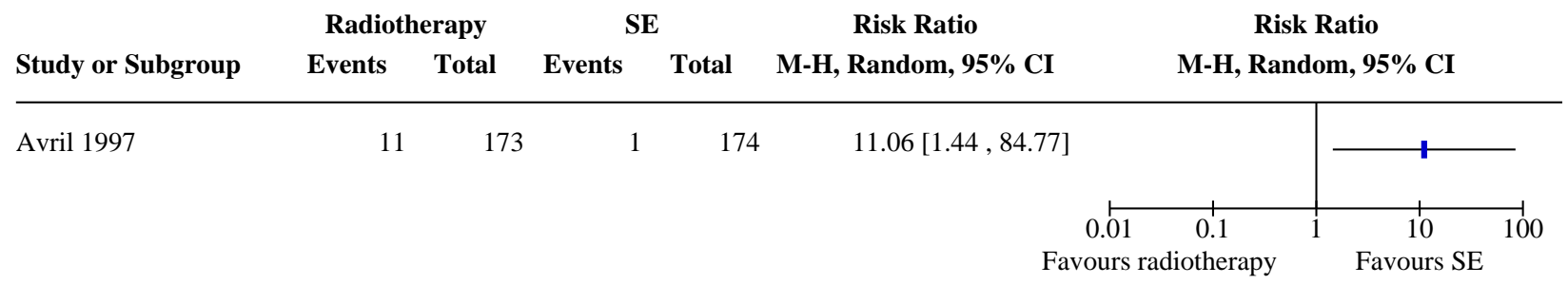

Analysis 3.3. Comparison 3: Radiotherapy vs surgical excision (with or without frozen section margin control), Outcome 3: Cosmetic outcome (good)

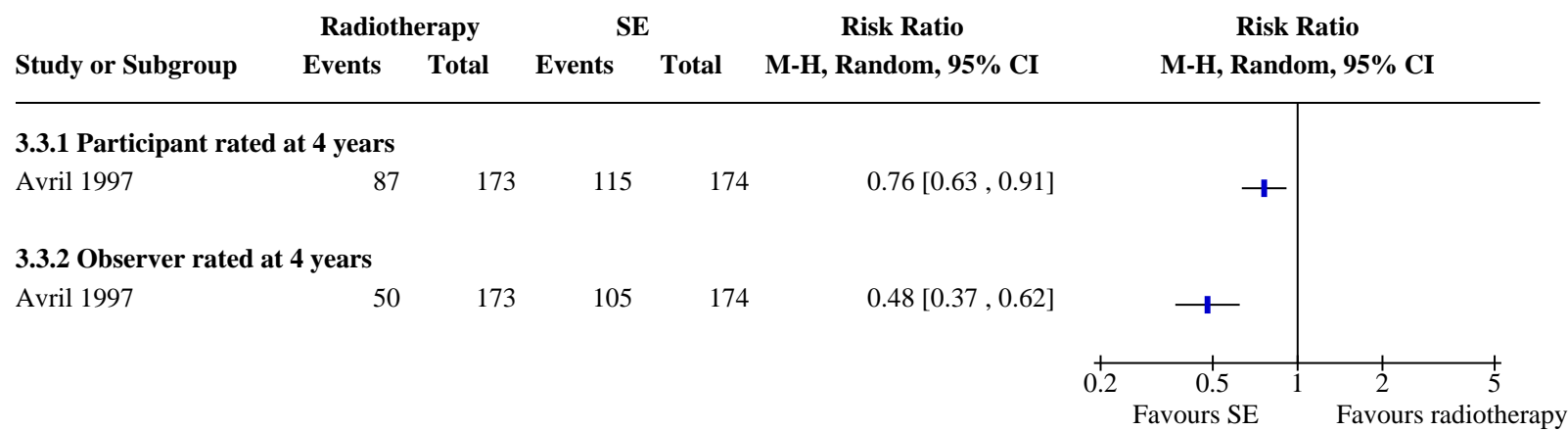

\section{Comparison 4. Curettage vs surgical excision}

\begin{tabular}{lllll}
\hline Outcome or subgroup title & $\begin{array}{l}\text { No. of } \\
\text { studies }\end{array}$ & $\begin{array}{l}\text { No. of par- } \\
\text { ticipants }\end{array}$ & Statistical method & Effect size \\
\hline 4.1 Recurrence at 2 years (lesions) & 1 & Risk Ratio (M-H, Random, 95\% Cl) & Totals not selected \\
\hline
\end{tabular}


Analysis 4.1. Comparison 4: Curettage vs surgical excision, Outcome 1: Recurrence at 2 years (lesions)

$$
\begin{array}{llll}
\text { Curettage } & \text { SE } & \text { Risk Ratio } & \text { Risk Ratio }
\end{array}
$$

Study or Subgroup $\quad$ Events $\quad$ Total $\quad$ Events $\quad$ Total $\quad$ M-H, Random, 95\% CI $\quad$ M-H, Random, 95\% CI

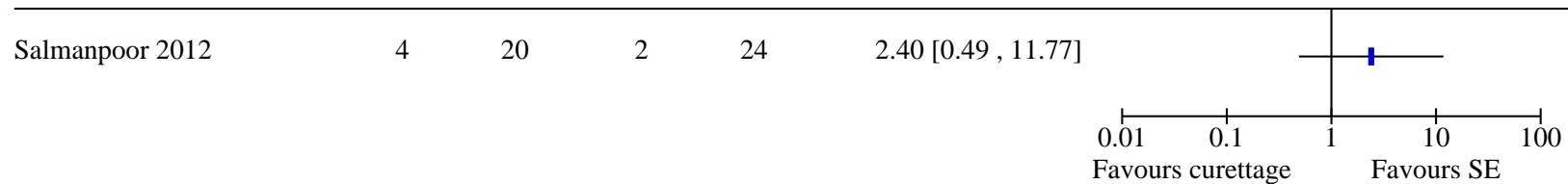

\section{Comparison 5. Curettage and cautery vs surgical excision}

\begin{tabular}{lllll}
\hline Outcome or subgroup title & $\begin{array}{l}\text { No. of } \\
\text { studies }\end{array}$ & $\begin{array}{l}\text { No. of par- } \\
\text { ticipants }\end{array}$ & Statistical method & Effect size \\
\hline 5.1 Recurrence at 2 years (lesions) & 1 & & Risk Ratio (M-H, Random, 95\% Cl) & Totals not selected \\
\hline
\end{tabular}

Analysis 5.1. Comparison 5: Curettage and cautery vs surgical excision, Outcome 1: Recurrence at 2 years (lesions)

$$
\text { C\&C SE Risk Ratio } \quad \text { Risk Ratio }
$$

Study or Subgroup Events Total Events Total M-H, Random, 95\% CI $\quad$ M-H, Random, 95\% CI

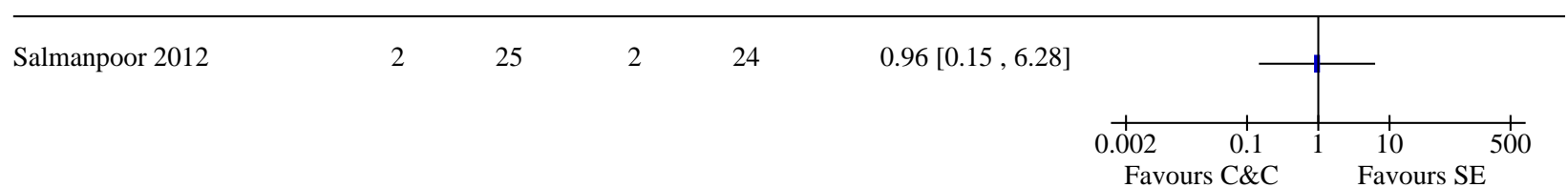

\section{Comparison 6. Curettage and cautery vs curettage}

\begin{tabular}{lllll}
\hline Outcome or subgroup title & $\begin{array}{l}\text { No. of } \\
\text { studies }\end{array}$ & $\begin{array}{l}\text { No. of par- } \\
\text { ticipants }\end{array}$ & Statistical method & Effect size \\
\hline 6.1 Recurrence at 2 years (lesions) & 1 & Risk Ratio (M-H, Random, 95\% Cl) & Totals not selected \\
\hline
\end{tabular}

Analysis 6.1. Comparison 6: Curettage and cautery vs curettage, Outcome 1: Recurrence at 2 years (lesions)

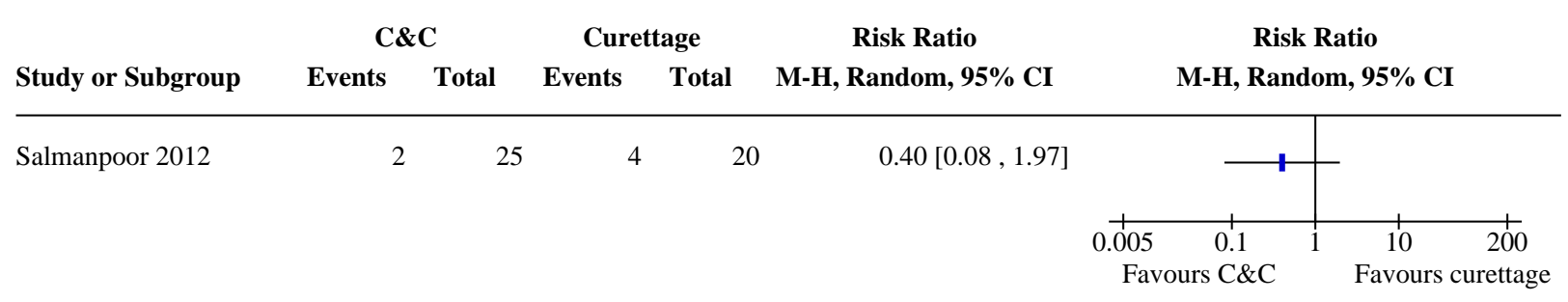


Comparison 7. Low dose radiotherapy vs high dose radiotherapy

\begin{tabular}{lllll}
\hline Outcome or subgroup title & $\begin{array}{l}\text { No. of } \\
\text { studies }\end{array}$ & $\begin{array}{l}\text { No. of par- } \\
\text { ticipants }\end{array}$ & Statistical method & Effect size \\
\hline 7.1 Early treatment failure & 1 & Risk Ratio (M-H, Random, 95\% Cl) & Totals not selected \\
\hline
\end{tabular}

Analysis 7.1. Comparison 7: Low dose radiotherapy vs high dose radiotherapy, Outcome 1: Early treatment failure

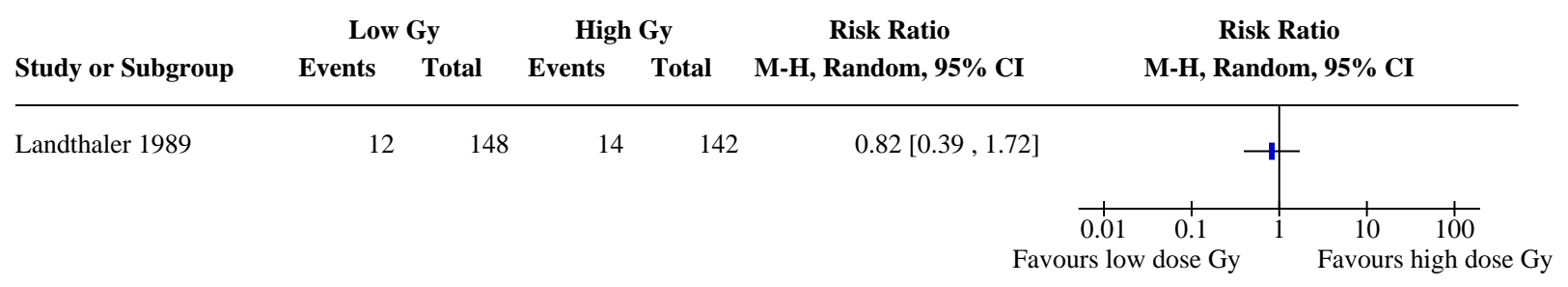

Comparison 8. Radiotherapy vs cryosurgery

\begin{tabular}{lllll}
\hline Outcome or subgroup title & $\begin{array}{l}\text { No. of } \\
\text { studies }\end{array}$ & $\begin{array}{l}\text { No. of par- } \\
\text { ticipants }\end{array}$ & Statistical method & Effect size \\
\hline 8.1 Recurrence at 2 years & 1 & Risk Ratio (M-H, Random, 95\% Cl) & Totals not selected \\
\hline
\end{tabular}

\section{Analysis 8.1. Comparison 8: Radiotherapy vs cryosurgery, Outcome 1: Recurrence at 2 years}

\begin{tabular}{|c|c|c|c|c|c|c|c|}
\hline \multirow{3}{*}{$\begin{array}{l}\text { Study or Subgroup } \\
\text { Hall } 1986\end{array}$} & \multicolumn{2}{|c|}{ Radiotherapy } & \multicolumn{2}{|c|}{ Cryosurgery } & \multirow{2}{*}{$\begin{array}{c}\text { Risk Ratio } \\
\text { M-H, Random, 95\% CI }\end{array}$} & \multirow{2}{*}{\multicolumn{2}{|c|}{$\begin{array}{c}\text { Risk Ratio } \\
\text { M-H, Random, 95\% CI }\end{array}$}} \\
\hline & Events & Total & Events & Total & & & \\
\hline & 2 & 49 & 17 & 44 & $0.11[0.03,0.43]$ & 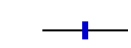 & \\
\hline & & & & & Fav & $\begin{array}{c}0.1 \\
\text { diotherapy }\end{array}$ & $\begin{array}{c}10 \\
\text { Favours c }\end{array}$ \\
\hline
\end{tabular}

Comparison 9. Curettage and cryosurgery vs surgical excision

\begin{tabular}{lllll}
\hline Outcome or subgroup title & $\begin{array}{l}\text { No. of } \\
\text { studies }\end{array}$ & $\begin{array}{l}\text { No. of } \\
\text { partici- } \\
\text { pants }\end{array}$ & Statistical method & Effect size \\
\hline $\begin{array}{l}\text { 9.1 Recurrence at 3 years (le- } \\
\text { sions) }\end{array}$ & 1 & Risk Ratio (M-H, Random, 95\% Cl) & Totals not selected \\
\hline $\begin{array}{l}\text { 9.2 Recurrence at 5 years (le- } \\
\text { sions) }\end{array}$ & 1 & Risk Ratio (M-H, Random, $95 \% \mathrm{Cl})$ & Totals not selected \\
\hline 9.3 Cosmetic outcome (good) & 1 & Risk Ratio (M-H, Random, $95 \% \mathrm{Cl})$ & Totals not selected \\
\hline 9.3 .1 Participant rated at 1 year & 1 & Risk Ratio (M-H, Random, $95 \% \mathrm{Cl})$ & Totals not selected \\
\hline \hline
\end{tabular}




\begin{tabular}{llll}
\hline Outcome or subgroup title & $\begin{array}{l}\text { No. of } \\
\text { studies }\end{array}$ & $\begin{array}{l}\text { No. of } \\
\text { partici- } \\
\text { pants }\end{array}$ & Statistical method size \\
\hline
\end{tabular}

9.3.2 Obsever rated at 1 year $\quad 1 \quad$ Risk Ratio $(\mathrm{M}-\mathrm{H}$, Random, $95 \% \mathrm{Cl}) \quad$ Totals not selected

Analysis 9.1. Comparison 9: Curettage and cryosurgery vs surgical excision, Outcome 1: Recurrence at 3 years (lesions)

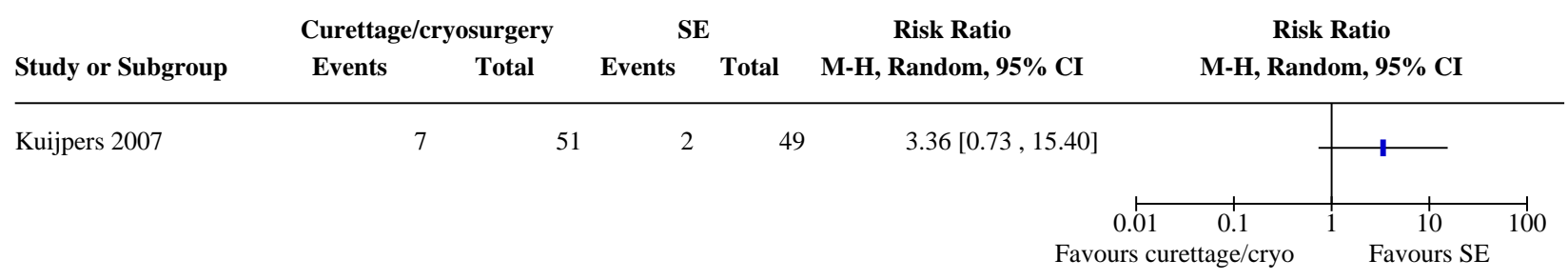

Analysis 9.2. Comparison 9: Curettage and cryosurgery vs surgical excision, Outcome 2: Recurrence at 5 years (lesions)

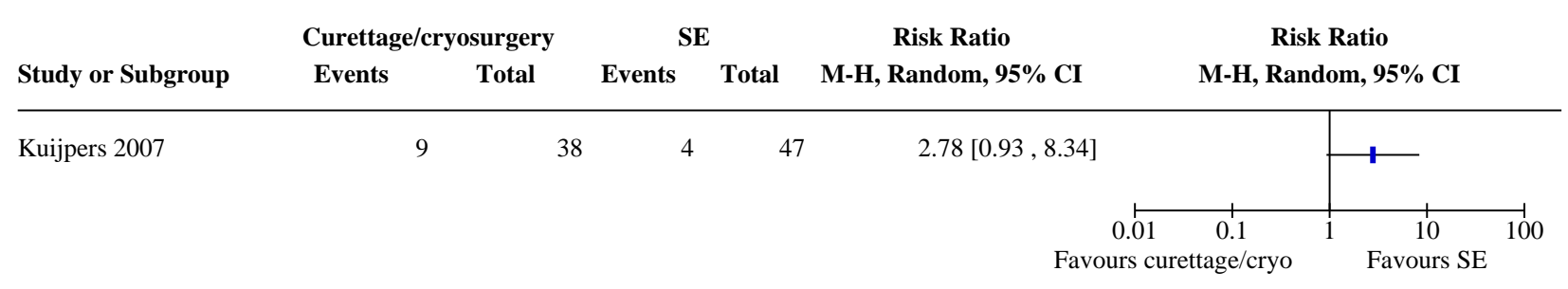

\section{Analysis 9.3. Comparison 9: Curettage and cryosurgery vs surgical excision, Outcome 3: Cosmetic outcome (good)}

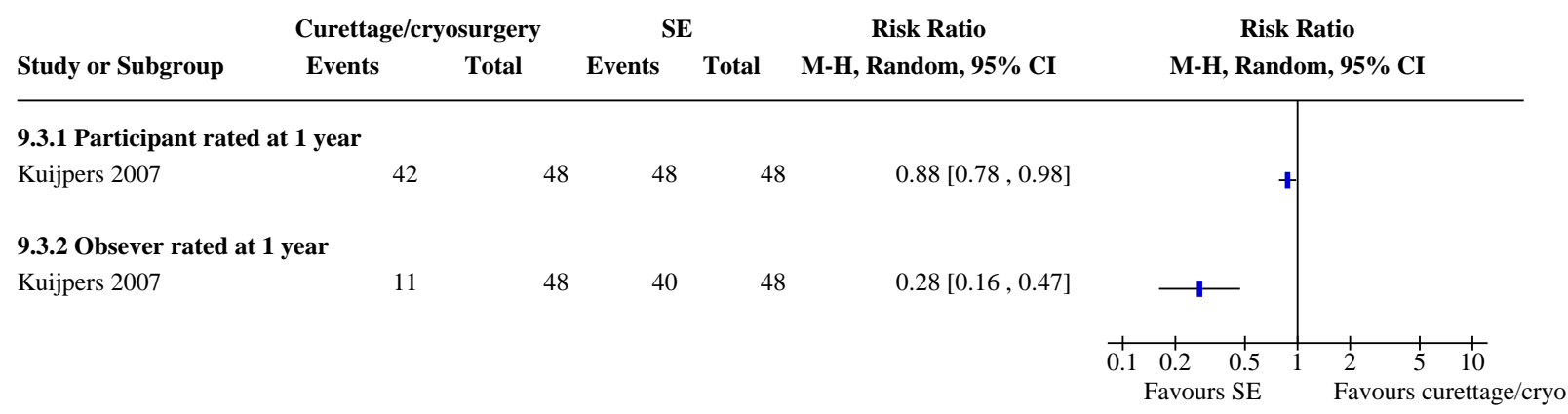

\section{Comparison 10. MAL-PDT vs surgical excision}

\begin{tabular}{lllll}
\hline Outcome or subgroup title & $\begin{array}{l}\text { No. of } \\
\text { studies }\end{array}$ & $\begin{array}{l}\text { No. of } \\
\text { partici- } \\
\text { pants }\end{array}$ & Statistical method & Effect size \\
\hline 10.1 Recurrence at 3 years (lesions) & 1 & & Risk Ratio (M-H, Random, 95\% Cl) & Totals not selected \\
\hline
\end{tabular}




\begin{tabular}{llllll}
\hline Outcome or subgroup title & $\begin{array}{l}\text { No. of } \\
\text { studies }\end{array}$ & $\begin{array}{l}\text { No. of } \\
\text { partici- } \\
\text { pants }\end{array}$ & Statistical method & Effect size \\
\hline $\begin{array}{l}10.2 \text { Cosmetic outcome (good/excel- } \\
\text { lent) }\end{array}$ & 2 & & Risk Ratio (M-H, Random, 95\% Cl) & Subtotals only \\
\hline $\begin{array}{l}10.2 .1 \text { Participant rated at 1 year } \\
10.2 .2 \text { Observer rated 1 year }\end{array}$ & 2 & 309 & Risk Ratio (M-H, Random, 95\% Cl) & $1.18[1.09,1.27]$ \\
\hline $\begin{array}{l}10.3 \text { Pain } \\
10.4 \text { Early treatment failure (lesions) }\end{array}$ & 1 & 256 & Risk Ratio (M-H, Random, 95\% Cl) & $1.87[1.54,2.26]$ \\
\hline
\end{tabular}

Analysis 10.1. Comparison 10: MAL-PDT vs surgical excision, Outcome 1: Recurrence at 3 years (lesions)

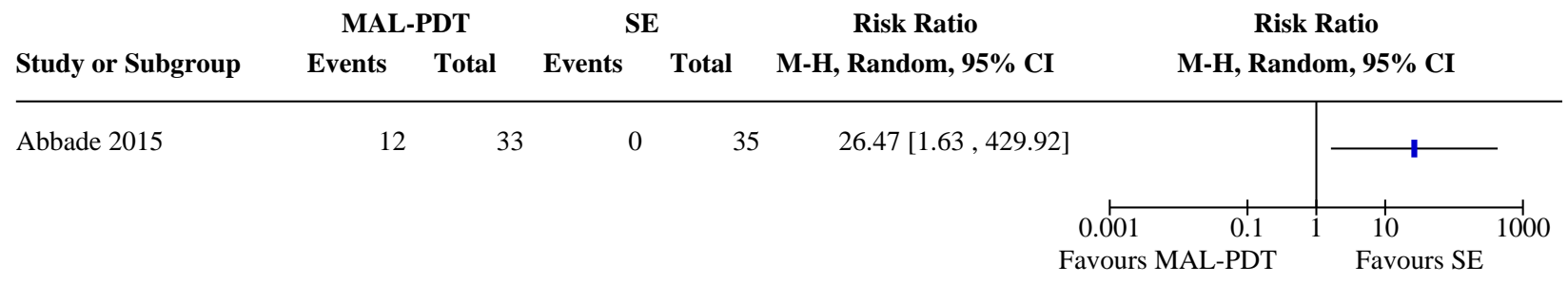

Analysis 10.2. Comparison 10: MAL-PDT vs surgical excision, Outcome 2: Cosmetic outcome (good/excellent)

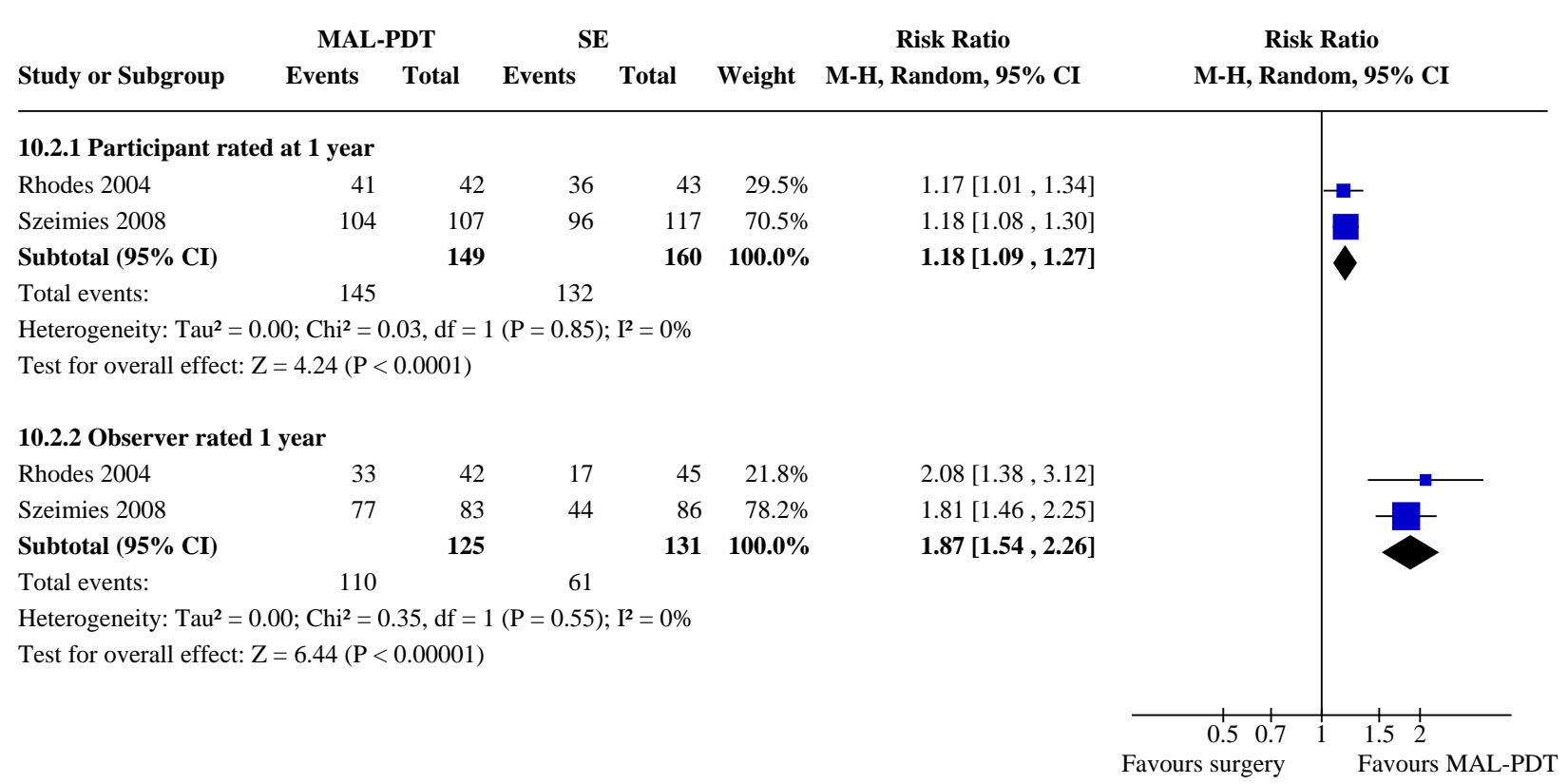


Analysis 10.3. Comparison 10: MAL-PDT vs surgical excision, Outcome 3: Pain

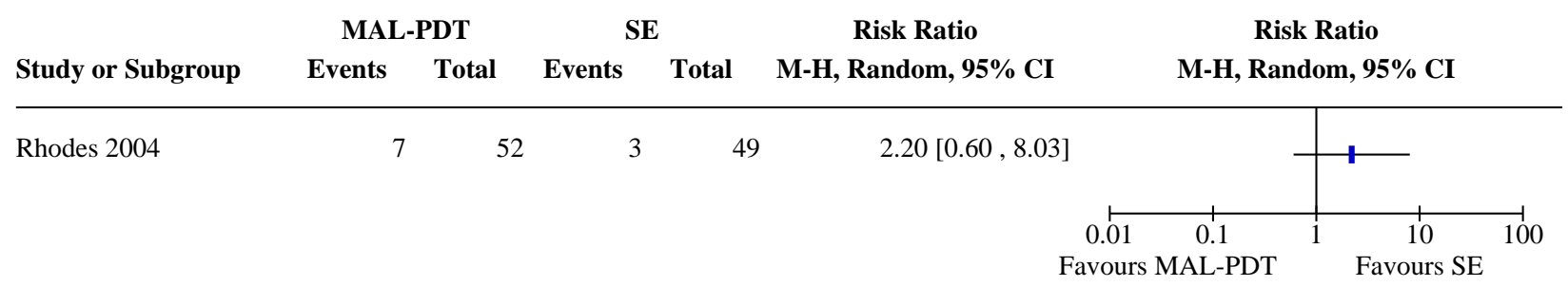

Analysis 10.4. Comparison 10: MAL-PDT vs surgical excision, Outcome 4: Early treatment failure (lesions)
MAL-PDT
SE
Risk Ratio
Risk Ratio

Study or Subgroup Events Total Events Total M-H, Random, 95\% CI M-H, Random, $95 \%$ CI

$\begin{array}{llllll}\text { Abbade } 2015 & 5 & 33 & 0 & 35 & 11.65[0.67,202.74]\end{array}$

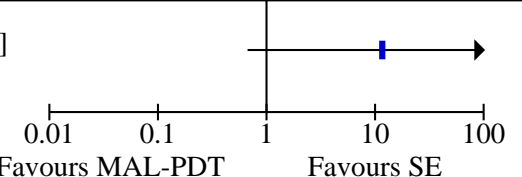

\section{Comparison 11. ALA-PDT vs surgical excision}

\begin{tabular}{lllll}
\hline Outcome or subgroup title & $\begin{array}{l}\text { No. of } \\
\text { studies }\end{array}$ & $\begin{array}{l}\text { No. of } \\
\text { partici- } \\
\text { pants }\end{array}$ & Statistical method & Effect size \\
\hline 11.1 Recurrence at 3 years (lesions) & 1 & Risk Ratio (M-H, Random, 95\% Cl) & Totals not selected \\
\hline 11.2 Recurrence at 5 years (lesions) & 1 & Risk Ratio (M-H, Random, 95\% Cl) & Totals not selected \\
\hline 11.3 Early treatment failure & 1 & Risk Ratio (M-H, Random, 95\% Cl) & Totals not selected \\
\hline
\end{tabular}

Analysis 11.1. Comparison 11: ALA-PDT vs surgical excision, Outcome 1: Recurrence at 3 years (lesions)

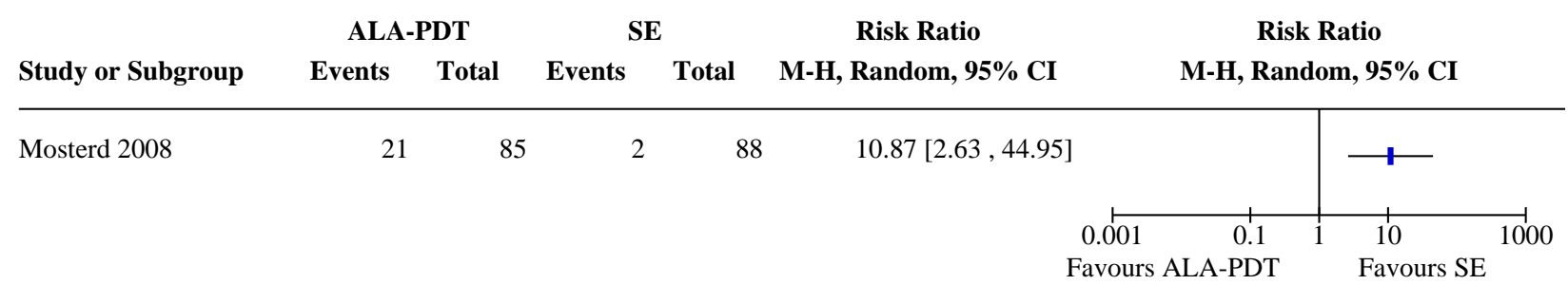


Analysis 11.2. Comparison 11: ALA-PDT vs surgical excision, Outcome 2: Recurrence at 5 years (lesions)
ALA-PDT
SE
Risk Ratio
Risk Ratio

Study or Subgroup Events Total Events Total M-H, Random, 95\% CI M-H, Random, $95 \%$ CI

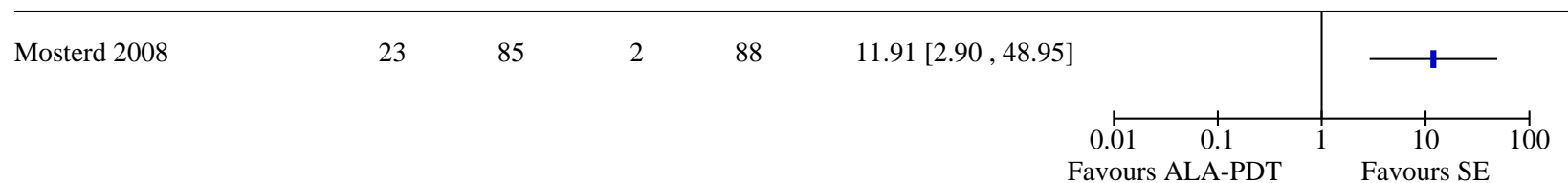

\section{Analysis 11.3. Comparison 11: ALA-PDT vs surgical excision, Outcome 3: Early treatment failure}

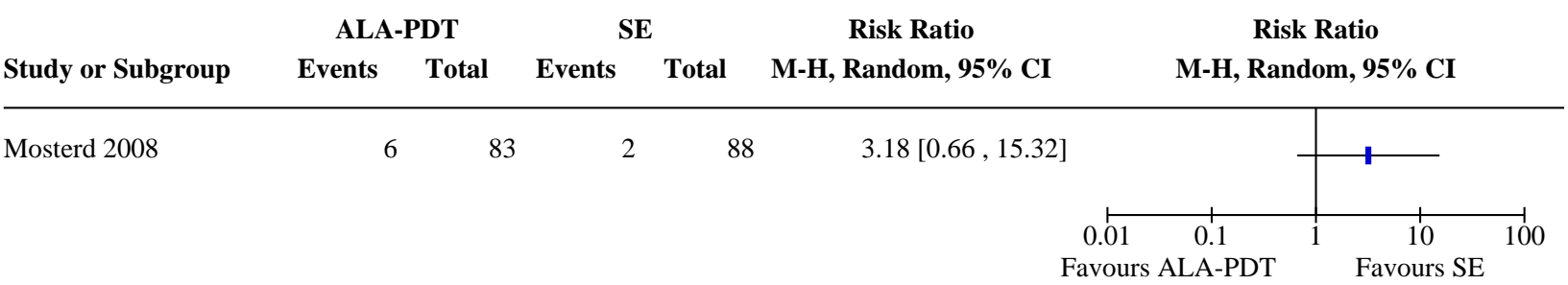

\section{Comparison 12. ALA-PDT vs MAL-PDT}

\begin{tabular}{|c|c|c|c|c|}
\hline Outcome or subgroup title & $\begin{array}{l}\text { No. of } \\
\text { studies }\end{array}$ & $\begin{array}{l}\text { No. of } \\
\text { partici- } \\
\text { pants }\end{array}$ & Statistical method & Effect size \\
\hline $\begin{array}{l}12.1 \text { Cosmetic outcome (good/very } \\
\text { good) }\end{array}$ & 2 & & Risk Ratio (M-H, Random, 95\% Cl) & Totals not selected \\
\hline 12.2 Pain (visual analogue score) & 2 & & Mean Difference (IV, Random, 95\% CI) & Subtotals only \\
\hline 12.2.1 Cycle 1 & 2 & 313 & Mean Difference (IV, Random, 95\% CI) & $0.56[-0.76,1.88]$ \\
\hline 12.2.2 Cycle 2 & 2 & 300 & Mean Difference (IV, Random, 95\% CI) & $0.43[-0.18,1.05]$ \\
\hline 12.3 Pain (numerical rating scale) & 1 & & Mean Difference (IV, Random, 95\% CI) & Totals not selected \\
\hline 12.3.1 Cycle 1 & 1 & & Mean Difference (IV, Random, 95\% CI) & Totals not selected \\
\hline 12.3.2 Cycle 2 & 1 & & Mean Difference (IV, Random, 95\% CI) & Totals not selected \\
\hline 12.4 Early treatment failure & 2 & & Risk Ratio (M-H, Random, 95\% Cl) & Totals not selected \\
\hline
\end{tabular}


Analysis 12.1. Comparison 12: ALA-PDT vs MAL-PDT, Outcome 1: Cosmetic outcome (good/very good)
ALA-PDT
MAL-PDT
Risk Ratio
Risk Ratio

Study or Subgroup Events Tota

Events Total

M-H, Random, 95\% CI

M-H, Random, 95\% CI

$\begin{array}{llllll}\text { Kessels } 2018 & 58 & 73 & 48 & 72 & 1.19[0.98,1.46] \\ \text { Morton } 2018 & 41 & 56 & 39 & 57 & 1.07[0.84,1.36]\end{array}$

\section{Analysis 12.2. Comparison 12: ALA-PDT vs MAL-PDT, Outcome 2: Pain (visual analogue score)}

\begin{tabular}{|c|c|c|c|c|c|c|c|c|c|}
\hline \multirow{2}{*}{\multicolumn{4}{|c|}{$\begin{array}{l}\text { ALA-PDT } \\
\end{array}$}} & \multicolumn{3}{|c|}{ MAL-PDT } & \multicolumn{2}{|r|}{ Mean Difference } & \multirow{2}{*}{$\begin{array}{c}\text { Mean Difference } \\
\text { IV, Random, 95\% CI }\end{array}$} \\
\hline & & & Total & Mean & SD & Total & Weight & IV, Random, 95\% CI & \\
\hline
\end{tabular}

\subsubsection{Cycle 1}

Kuijpers 2006

Morton 2018

Subtotal $(95 \%$ CI)

$\begin{array}{lllll}4.38 & 2.57 & 13 & 2.84 & 2.41\end{array}$

$19 \quad 32.2 \%$

$143 \quad 67.8 \%$

Subtotal (95\% CI)

$162100.0 \%$

$1.54[-0.23,3.31]$
$0.10[-0.44,0.64]$

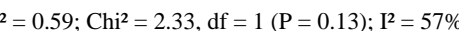

$0.56[-0.76,1.88]$

Test for overall effect: $\mathrm{Z}=0.84(\mathrm{P}=0.40)$

\subsubsection{Cycle 2}

Kuijpers 2006

Morton 2018

$4.83 \quad 3.21$

12

3.89

Subtotal (95\% CI)

150

$138 \quad 93.5 \%$

$0.40[-0.24,1.04]$

Heterogeneity: $\mathrm{Tau}^{2}=0.00 ; \mathrm{Chi}^{2}=0.18, \mathrm{df}=1(\mathrm{P}=0.67) ; \mathrm{I}^{2}=0 \%$

$150 \quad 100.0 \%$

$0.43[-0.18,1.05]$

Test for overall effect: $\mathrm{Z}=1.38(\mathrm{P}=0.17)$

, Random, 95\% CI

Analysis 12.3. Comparison 12: ALA-PDT vs MAL-PDT, Outcome 3: Pain (numerical rating scale)

\begin{tabular}{|c|c|c|c|c|c|c|c|c|}
\hline & \multicolumn{3}{|c|}{ ALA-PDT } & \multicolumn{3}{|c|}{ MAL-PDT } & Mean Difference & Mean Difference \\
\hline Study or Subgroup & Mean & SD & Total & Mean & SD & Total & IV, Random, $95 \%$ CI & IV, Random, $95 \%$ CI \\
\hline
\end{tabular}

12.3.1 Cycle 1

Kessels 2018

$\begin{array}{lllllll}1.88 & 2.36 & 82 & 2.25 & 2.54 & 79 & -0.37[-1.13,0.39]\end{array}$

12.3.2 Cycle 2

Kessels 2018

$\begin{array}{llllllll}\text { Kessels } 2018 & 3.36 & 2.57 & 82 & 2.48 & 2.57 & 79 & 0.88[0.09,1.67]\end{array}$

82

2.57

79

$0.88[0.09,1.67]$

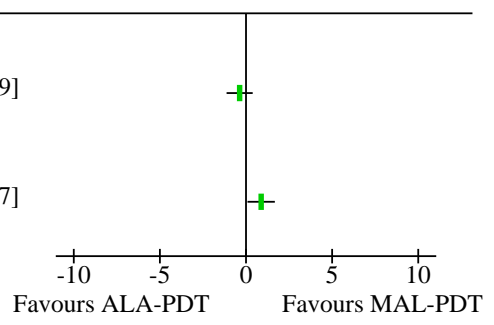


Analysis 12.4. Comparison 12: ALA-PDT vs MAL-PDT, Outcome 4: Early treatment failure

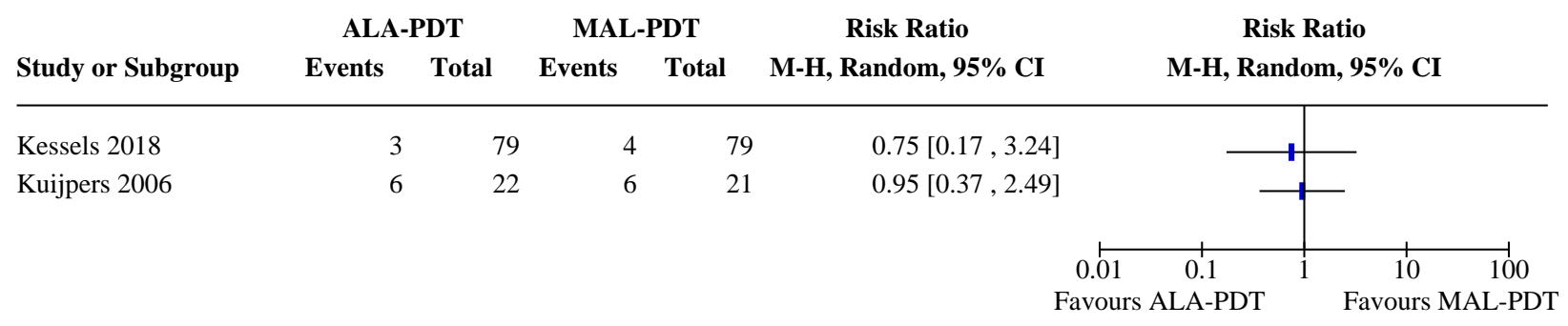

Comparison 13. BF-200 ALA-PDT vs MAL-PDT

\begin{tabular}{lllll}
\hline Outcome or subgroup title & $\begin{array}{l}\text { No. of } \\
\text { studies }\end{array}$ & $\begin{array}{l}\text { No. of par- } \\
\text { ticipants }\end{array}$ & Statistical method & Effect size \\
\hline 13.1 Early treatment failure & 1 & Risk Ratio (M-H, Random, 95\% Cl) & Totals not selected \\
\hline
\end{tabular}

Analysis 13.1. Comparison 13: BF-200 ALA-PDT vs MAL-PDT, Outcome 1: Early treatment failure

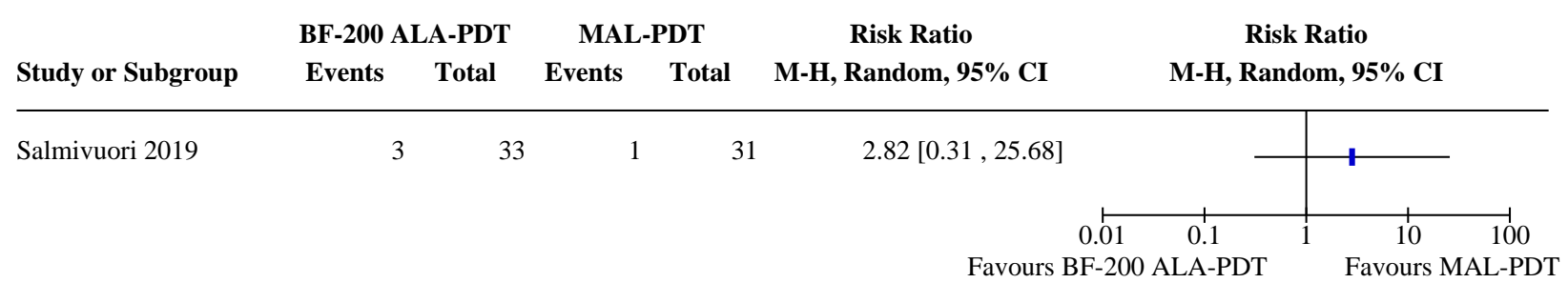

\section{Comparison 14. HAL-PDT vs MAL-PDT}

\begin{tabular}{lllll}
\hline Outcome or subgroup title & $\begin{array}{l}\text { No. of } \\
\text { studies }\end{array}$ & $\begin{array}{l}\text { No. of par- } \\
\text { ticipants }\end{array}$ & Statistical method & Effect size \\
\hline 14.1 Early treatment failure & 1 & Risk Ratio (M-H, Random, 95\% Cl) & Totals not selected \\
\hline
\end{tabular}

Analysis 14.1. Comparison 14: HAL-PDT vs MAL-PDT, Outcome 1: Early treatment failure

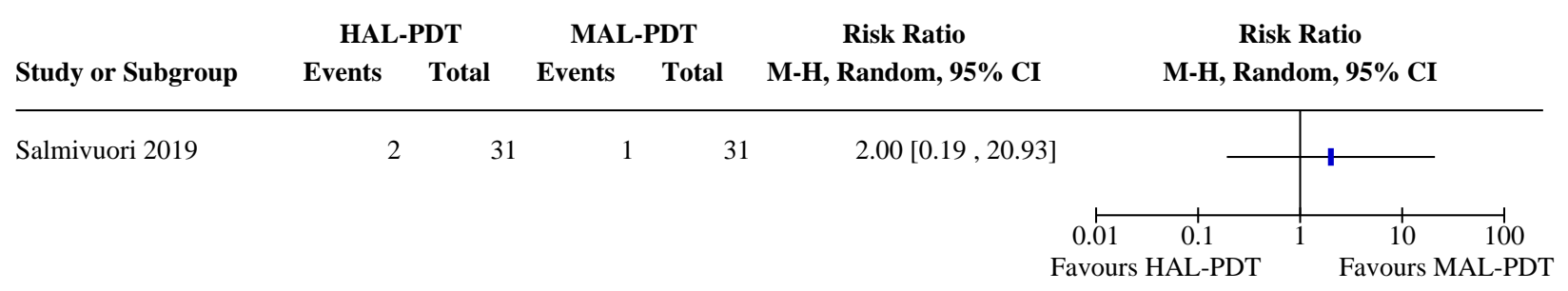


Comparison 15. HAL-PDT vs BF-200 ALA-PDT

\begin{tabular}{llll}
\hline Outcome or subgroup title & $\begin{array}{l}\text { No. of } \\
\text { studies }\end{array}$ & $\begin{array}{l}\text { No. of par- } \\
\text { ticipants }\end{array}$ & Statistical method \\
\hline 15.1 Early treatment failure & 1 & Risk Ratio (M-H, Random, 95\% Cl) & Totals not selected \\
\hline
\end{tabular}

Analysis 15.1. Comparison 15: HAL-PDT vs BF-200 ALA-PDT, Outcome 1: Early treatment failure

\begin{tabular}{|c|c|c|c|c|c|c|c|}
\hline \multirow[b]{2}{*}{ Study or Subgroup } & \multicolumn{2}{|c|}{ HAL-PDT } & \multicolumn{2}{|c|}{ BF-200-PDT } & \multirow{2}{*}{$\begin{array}{c}\text { Risk Ratio } \\
\text { M-H, Random, 95\% CI }\end{array}$} & \multirow{2}{*}{\multicolumn{2}{|c|}{$\begin{array}{c}\text { Risk Ratio } \\
\text { M-H, Random, 95\% CI }\end{array}$}} \\
\hline & Events & Total & Events & Total & & & \\
\hline \multirow[t]{2}{*}{ Salmivuori 2019} & 2 & 31 & 3 & 33 & $0.71[0.13,3.97]$ & 1 & - \\
\hline & & & & & & $\begin{array}{c}0.1 \\
\text { HAL-PDT }\end{array}$ & $\begin{array}{c}10 \\
\text { Favours B }\end{array}$ \\
\hline
\end{tabular}

\section{Comparison 16. MAL-PDT vs cryosurgery}

\begin{tabular}{|c|c|c|c|c|}
\hline Outcome or subgroup title & $\begin{array}{l}\text { No. of } \\
\text { studies }\end{array}$ & $\begin{array}{l}\text { No. of } \\
\text { partici- } \\
\text { pants }\end{array}$ & Statistical method & Effect size \\
\hline $\begin{array}{l}16.1 \text { Recurrence at } 3 \text { years (le- } \\
\text { sions) }\end{array}$ & 1 & & Risk Ratio (M-H, Random, 95\% Cl) & Totals not selected \\
\hline $\begin{array}{l}16.2 \text { Recurrence at } 5 \text { years (le- } \\
\text { sions) }\end{array}$ & 1 & & Risk Ratio (M-H, Random, 95\% Cl) & Totals not selected \\
\hline $\begin{array}{l}16.3 \text { Cosmetic outcome (good/ } \\
\text { excellent) }\end{array}$ & 1 & & Risk Ratio (M-H, Random, 95\% Cl) & Totals not selected \\
\hline $\begin{array}{l}\text { 16.3.1 Participant rated at } 1 \\
\text { year }\end{array}$ & 1 & & Risk Ratio (M-H, Random, 95\% Cl) & Totals not selected \\
\hline 16.3.2 Observer rated at 1 year & 1 & & Risk Ratio (M-H, Random, 95\% Cl) & Totals not selected \\
\hline 16.4 Pain & 1 & & Risk Ratio (M-H, Random, 95\% Cl) & Totals not selected \\
\hline $\begin{array}{l}16.5 \text { Early treatment failure (le- } \\
\text { sions) }\end{array}$ & 1 & & Risk Ratio (M-H, Random, 95\% Cl) & Totals not selected \\
\hline
\end{tabular}


Analysis 16.1. Comparison 16: MAL-PDT vs cryosurgery, Outcome 1: Recurrence at 3 years (lesions)

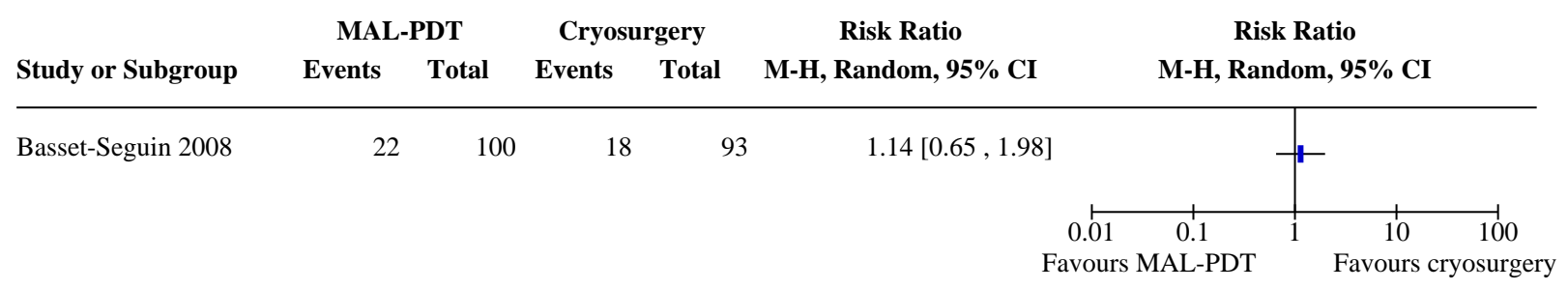

Analysis 16.2. Comparison 16: MAL-PDT vs cryosurgery, Outcome 2: Recurrence at 5 years (lesions)

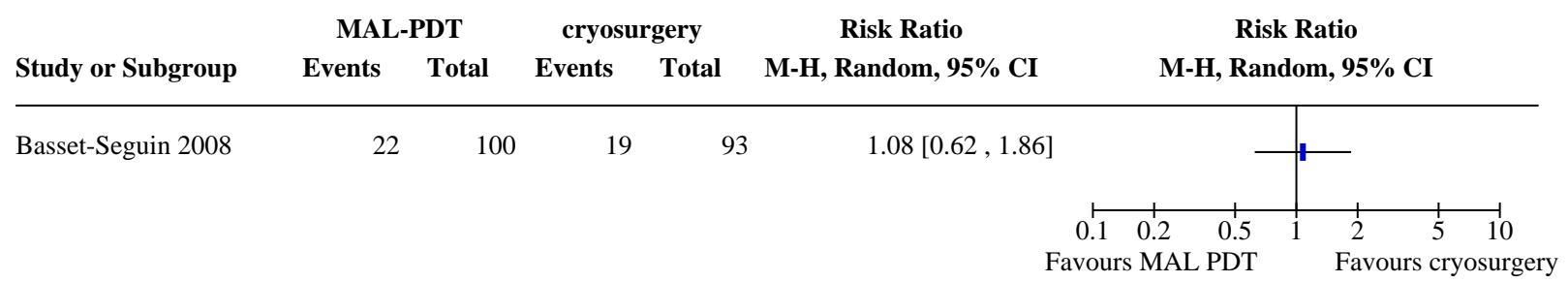

Analysis 16.3. Comparison 16: MAL-PDT vs cryosurgery, Outcome 3: Cosmetic outcome (good/excellent)
PDT
cryosurgery
Risk Ratio
Risk Ratio

Study or Subgroup $\quad$ Events $\quad$ Total $\quad$ Events $\quad$ Total $\quad$ M-H, Random, 95\% CI $\quad$ M-H, Random, 95\% CI

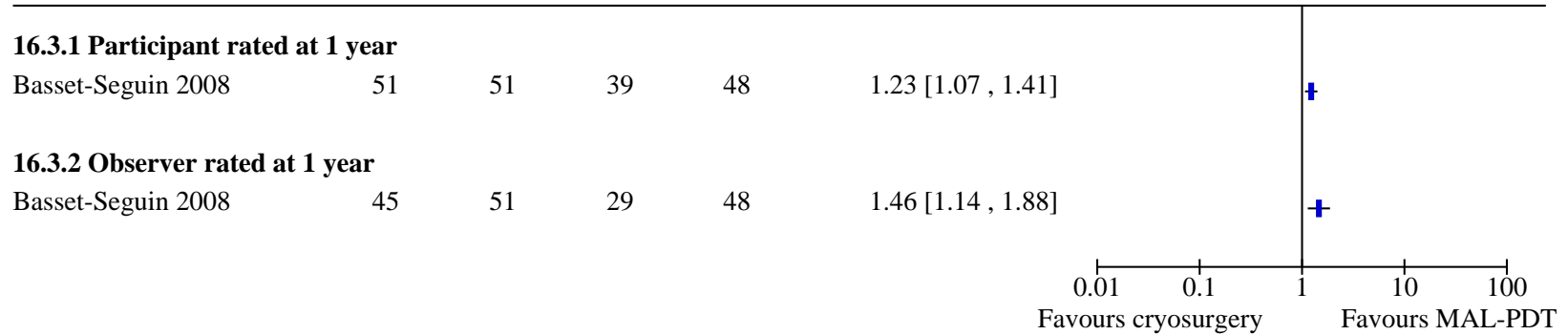

Analysis 16.4. Comparison 16: MAL-PDT vs cryosurgery, Outcome 4: Pain

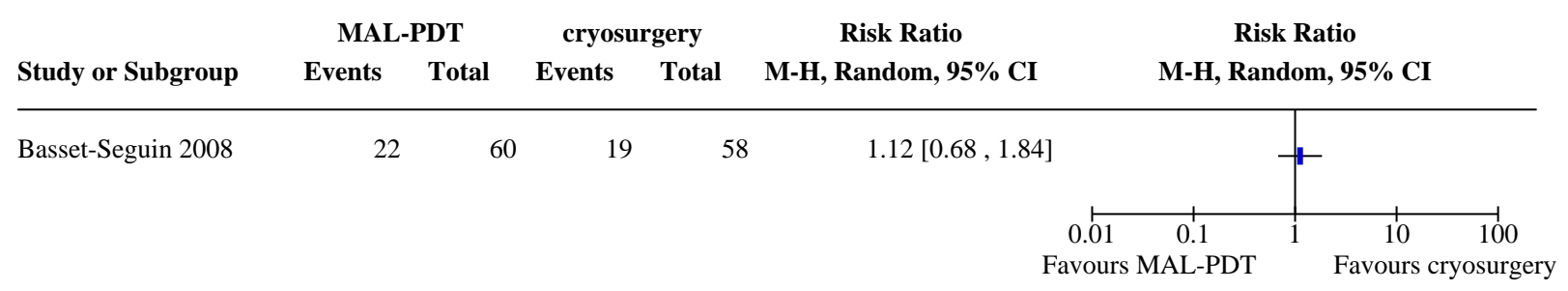


Analysis 16.5. Comparison 16: MAL-PDT vs cryosurgery, Outcome 5: Early treatment failure (lesions)

\begin{tabular}{|c|c|c|c|c|c|c|c|c|}
\hline \multirow{3}{*}{$\begin{array}{l}\text { Study or Subgroup } \\
\text { Basset-Seguin } 2008\end{array}$} & \multicolumn{2}{|c|}{ MAL PDT } & \multicolumn{2}{|c|}{ cryosurgery } & \multirow{2}{*}{$\begin{array}{c}\text { Risk Ratio } \\
\text { M-H, Random, } 95 \% \text { CI }\end{array}$} & \multirow{2}{*}{\multicolumn{2}{|c|}{$\begin{array}{c}\text { Risk Ratio } \\
\text { M-H, Random, 95\% CI }\end{array}$}} & \\
\hline & Events & Total & Events & Total & & & & \\
\hline & 3 & 103 & 5 & 98 & $0.57[0.14,2.33]$ & 1 & - & \\
\hline & & & & & & $0 . \mathfrak{\longmapsto}^{0}$ & 10 & 100 \\
\hline & & & & & & Favours PDT & Favours c & surgery \\
\hline
\end{tabular}

Comparison 17. ALA-PDT vs cryosurgery

\begin{tabular}{lllll}
\hline Outcome or subgroup title & $\begin{array}{l}\text { No. of } \\
\text { studies }\end{array}$ & $\begin{array}{l}\text { No. of } \\
\text { partici- } \\
\text { pants }\end{array}$ & Statistical method & Effect size \\
\hline $\begin{array}{l}17.1 \text { Cosmetic outcome (good/ex- } \\
\text { cellent) }\end{array}$ & 1 & & Risk Ratio (M-H, Random, 95\% Cl) & Totals not selected \\
\hline 17.2 Pain & 1 & & Mean Difference (IV, Random, 95\% Cl) & Totals not selected \\
\hline
\end{tabular}

Analysis 17.1. Comparison 17: ALA-PDT vs cryosurgery, Outcome 1: Cosmetic outcome (good/excellent)

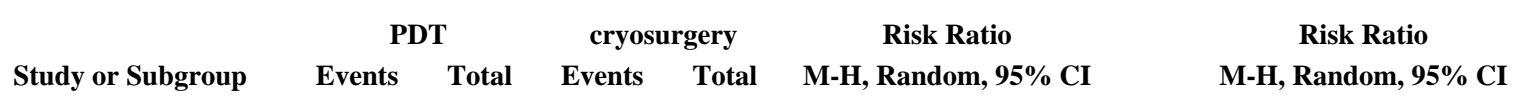

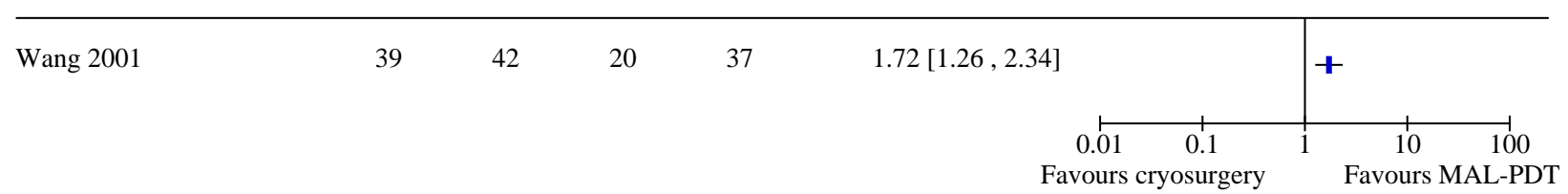

Analysis 17.2. Comparison 17: ALA-PDT vs cryosurgery, Outcome 2: Pain

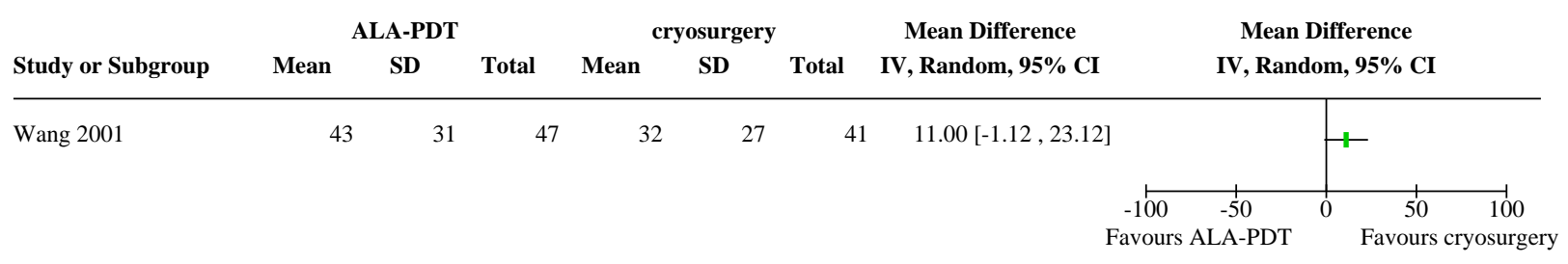

Comparison 18. Imiquimod cream vs MAL-PDT

\begin{tabular}{lllll}
\hline Outcome or subgroup title & $\begin{array}{l}\text { No. of } \\
\text { studies }\end{array}$ & $\begin{array}{l}\text { No. of } \\
\text { partici- } \\
\text { pants }\end{array}$ & Statistical method & Effect size \\
\hline 18.1 Recurrence at 3 years & 1 & Risk Ratio (M-H, Random, 95\% Cl) & Totals not selected \\
\hline 18.2 Recurrence at 5 years & 1 & Risk Ratio (M-H, Random, 95\% Cl) & Totals not selected \\
\hline
\end{tabular}




\begin{tabular}{lllll}
\hline Outcome or subgroup title & $\begin{array}{l}\text { No. of } \\
\text { studies }\end{array}$ & $\begin{array}{l}\text { No. of } \\
\text { partici- } \\
\text { pants }\end{array}$ & Statistical method & Effect size \\
\hline $\begin{array}{l}18.3 \text { Cosmetic outcome (good/ex- } \\
\text { cellent, observer rated at 1 year) }\end{array}$ & 1 & Risk Ratio (M-H, Random, 95\% Cl) & Totals not selected \\
\hline 18.4 Pain (moderate/severe) & 1 & Risk Ratio (M-H, Random, 95\% Cl) & Totals not selected \\
\hline 18.5 Early treatment failure & 1 & Risk Ratio (M-H, Random, 95\% Cl) & Totals not selected \\
\hline
\end{tabular}

Analysis 18.1. Comparison 18: Imiquimod cream vs MAL-PDT, Outcome 1: Recurrence at 3 years

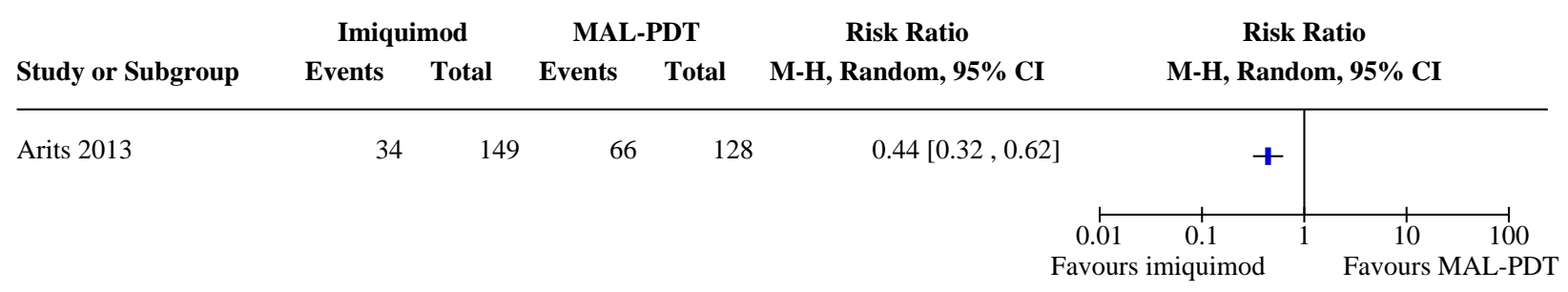

Analysis 18.2. Comparison 18: Imiquimod cream vs MAL-PDT, Outcome 2: Recurrence at 5 years

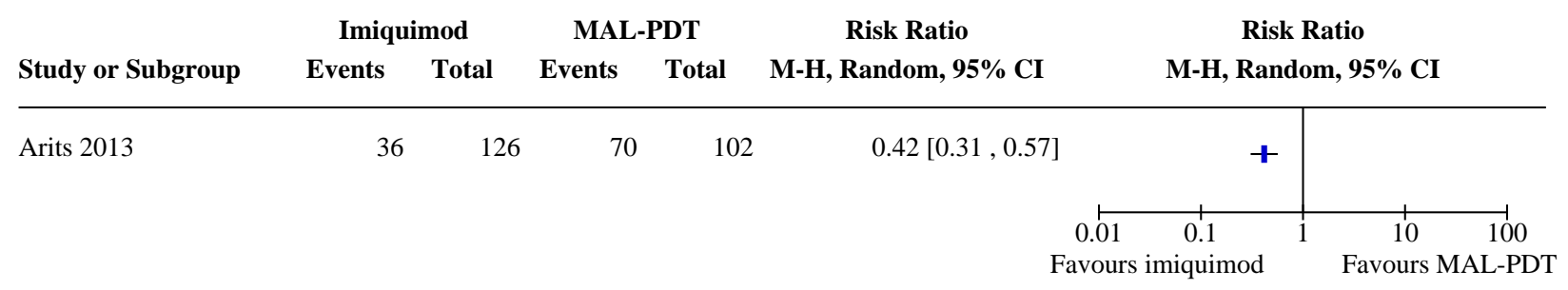

Analysis 18.3. Comparison 18: Imiquimod cream vs MAL-PDT, Outcome 3: Cosmetic outcome (good/excellent, observer rated at 1 year)

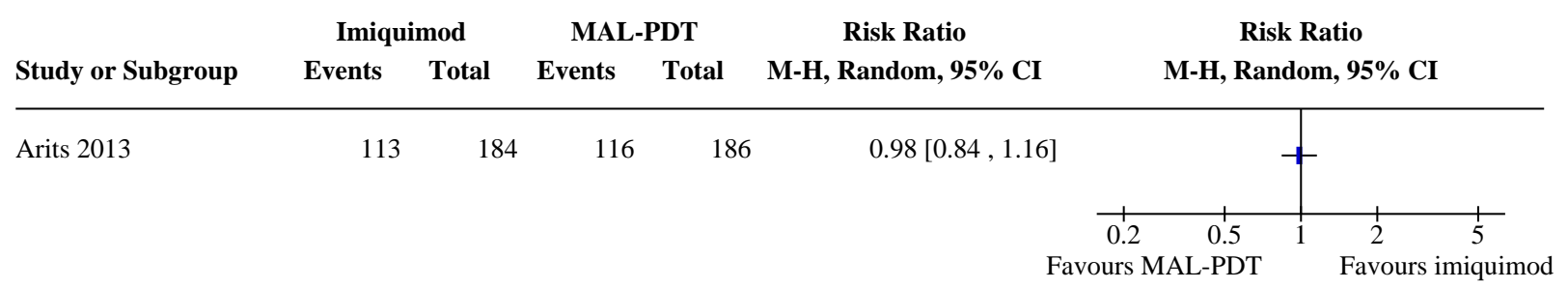


Analysis 18.4. Comparison 18: Imiquimod cream vs MAL-PDT, Outcome 4: Pain (moderate/severe)

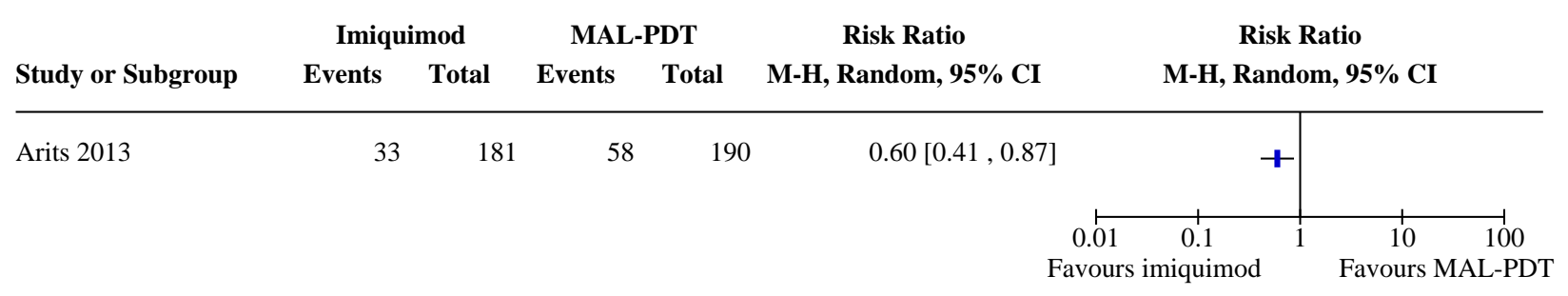

Analysis 18.5. Comparison 18: Imiquimod cream vs MAL-PDT, Outcome 5: Early treatment failure

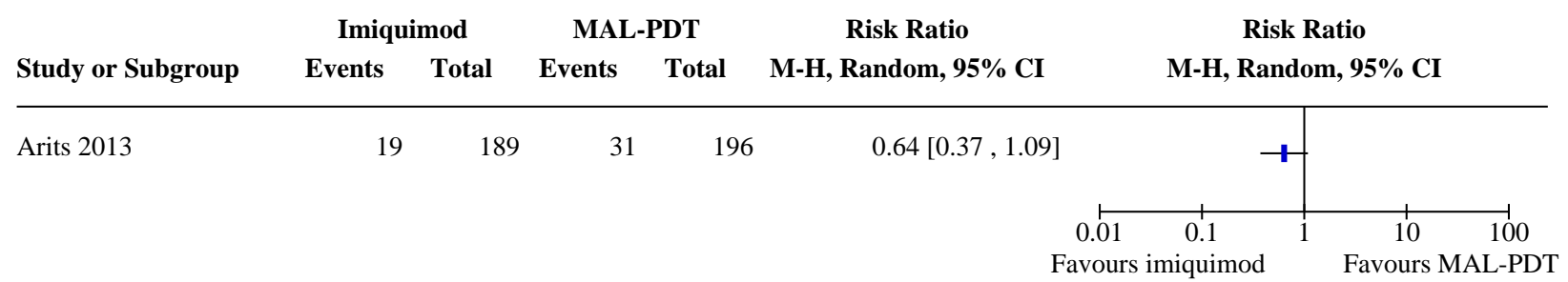

Comparison 19. 5-FU cream vs MAL-PDT

\begin{tabular}{lllll}
\hline Outcome or subgroup title & $\begin{array}{l}\text { No. of } \\
\text { studies }\end{array}$ & $\begin{array}{l}\text { No. of } \\
\text { partici- } \\
\text { pants }\end{array}$ & Statistical method & Effect size \\
\hline 19.1 Recurrence at 3 years & 1 & Risk Ratio (M-H, Random, 95\% Cl) & Totals not selected \\
\hline 19.2 Recurrence at 5 years & 1 & Risk Ratio (M-H, Random, 95\% Cl) & Totals not selected \\
\hline $\begin{array}{l}19.3 \text { Cosmetic outcome (good/ex- } \\
\text { cellent, observer rated at 1 year) }\end{array}$ & 1 & Risk Ratio (M-H, Random, 95\% Cl) & Totals not selected \\
\hline 19.4 Pain (moderate/severe) & 1 & Risk Ratio (M-H, Random, 95\% Cl) & Totals not selected \\
\hline 19.5 Early treatment failure & 1 & Risk Ratio (M-H, Random, 95\% Cl) & Totals not selected \\
\hline
\end{tabular}

Analysis 19.1. Comparison 19: 5-FU cream vs MAL-PDT, Outcome 1: Recurrence at 3 years

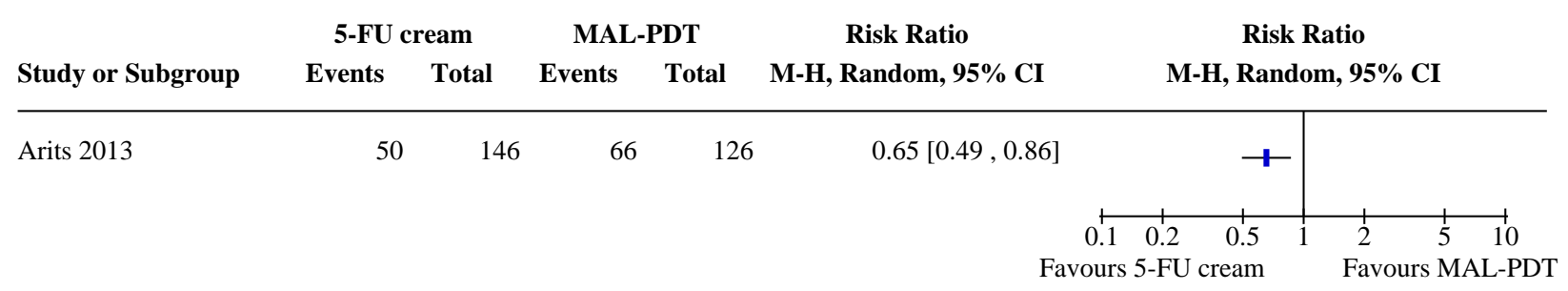


Analysis 19.2. Comparison 19: 5-FU cream vs MAL-PDT, Outcome 2: Recurrence at 5 years

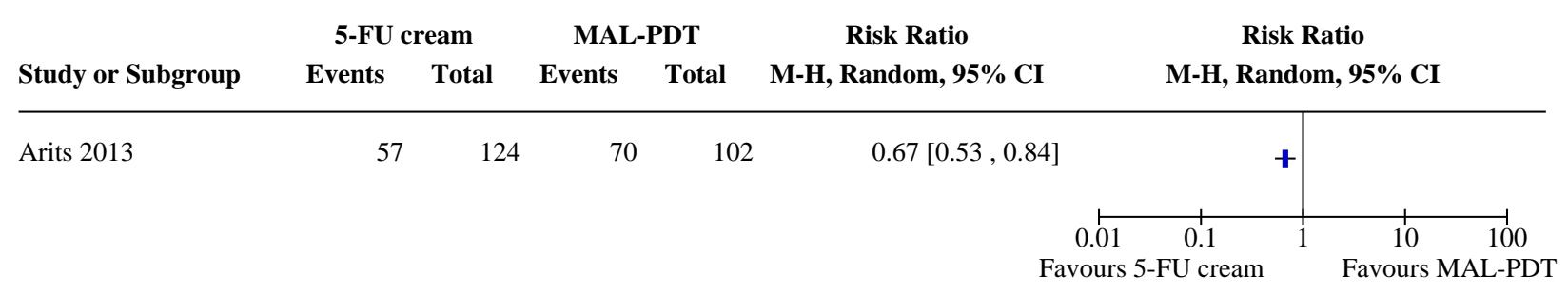

\section{Analysis 19.3. Comparison 19: 5-FU cream vs MAL-PDT, Outcome} 3: Cosmetic outcome (good/excellent, observer rated at 1 year)

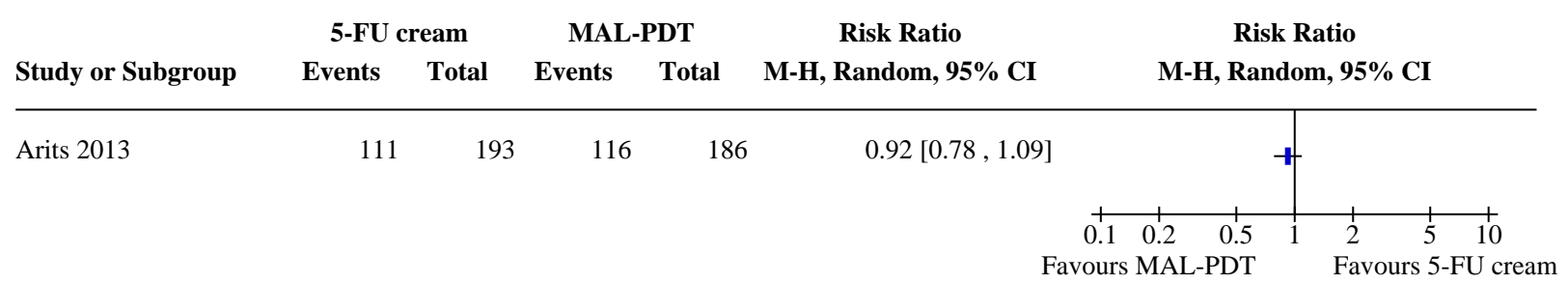

Analysis 19.4. Comparison 19: 5-FU cream vs MAL-PDT, Outcome 4: Pain (moderate/severe)

\begin{tabular}{|c|c|c|c|c|c|c|}
\hline & 5-FU & eam & MAI & DT & Risk Ratio & Risk Ratio \\
\hline Study or Subgroup & Events & Total & Events & Total & M-H, Random, 95\% CI & M-H, Random, 95\% CI \\
\hline
\end{tabular}

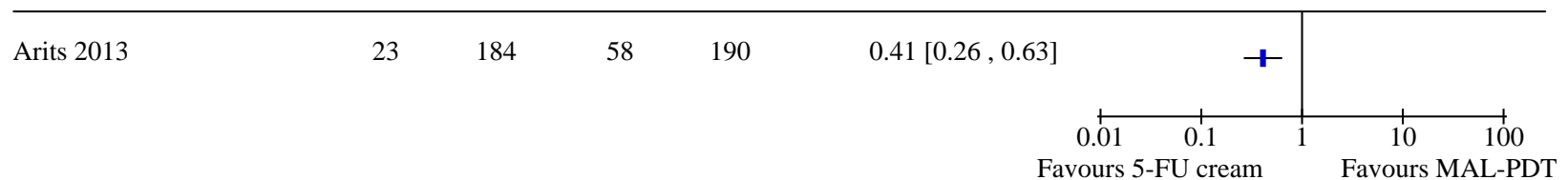

Analysis 19.5. Comparison 19: 5-FU cream vs MAL-PDT, Outcome 5: Early treatment failure

\begin{tabular}{|c|c|c|c|c|c|c|c|}
\hline \multirow{3}{*}{$\begin{array}{l}\text { Study or Subgroup } \\
\text { Arits } 2013\end{array}$} & \multicolumn{2}{|c|}{ 5-FU cream } & \multicolumn{2}{|c|}{ MAL-PDT } & \multirow{2}{*}{$\begin{array}{c}\text { Risk Ratio } \\
\text { M-H, Random, 95\% CI }\end{array}$} & \multirow{2}{*}{\multicolumn{2}{|c|}{$\begin{array}{c}\text { Risk Ratio } \\
\text { M-H, Random, } 95 \% \text { CI }\end{array}$}} \\
\hline & Events & Total & Events & Total & & & \\
\hline & 24 & 198 & 31 & 196 & $0.77[0.47,1.26]$ & + & \\
\hline
\end{tabular}

\section{Comparison 20. Laser ALA-PDT vs broadband light ALA-PDT}

\begin{tabular}{lllll}
\hline Outcome or subgroup title & $\begin{array}{l}\text { No. of } \\
\text { studies }\end{array}$ & $\begin{array}{l}\text { No. of } \\
\text { partici- } \\
\text { pants }\end{array}$ & Statistical method & Effect size \\
\hline $\begin{array}{l}20.1 \text { Cosmetic outcome (good/excellent, } \\
\text { observer rated at } 6 \text { months) }\end{array}$ & 1 & Risk Ratio (M-H, Random, 95\% Cl) & Subtotals only \\
\hline
\end{tabular}




\begin{tabular}{lllll}
\hline Outcome or subgroup title & $\begin{array}{l}\text { No. of } \\
\text { studies }\end{array}$ & $\begin{array}{l}\text { No. of } \\
\text { partici- } \\
\text { pants }\end{array}$ & Statistical method & Effect size \\
\hline 20.2 Pain & 1 & Risk Ratio (M-H, Random, 95\% Cl) & Totals not selected \\
\hline $\begin{array}{l}\text { 20.2.1 Discomfort during/after illumina- } \\
\text { tion }\end{array}$ & 1 & Risk Ratio (M-H, Random, 95\% Cl) & Totals not selected \\
\hline $\begin{array}{l}\text { 20.2.2 Discomfort during 1st week after } \\
\text { treatment }\end{array}$ & 1 & Risk Ratio (M-H, Random, 95\% Cl) & Totals not selected \\
\hline
\end{tabular}

Analysis 20.1. Comparison 20: Laser ALA-PDT vs broadband light ALA-PDT, Outcome 1: Cosmetic outcome (good/excellent, observer rated at 6 months)

\begin{tabular}{|c|c|c|c|c|c|c|c|}
\hline \multirow[b]{2}{*}{ Study or Subgroup } & \multicolumn{2}{|c|}{ Laser } & \multicolumn{2}{|c|}{ Broad band } & \multirow{2}{*}{$\begin{array}{c}\text { Risk Ratio } \\
\text { M-H, Random, 95\% CI }\end{array}$} & \multirow{2}{*}{\multicolumn{2}{|c|}{$\begin{array}{c}\text { Risk Ratio } \\
\text { M-H, Random, 95\% CI }\end{array}$}} \\
\hline & Events & Total & Events & Total & & & \\
\hline Soler 2000 & 80 & 95 & 102 & 110 & $0.91[0.82,1.01]$ & $H$ & \\
\hline & & & & & $\mathrm{Fa}$ & $\begin{array}{cc}0.5 & 0.7 \\
\text { 3road band }\end{array}$ & $\begin{array}{cc}1.5 \quad 2 \\
\text { Favours 1 }\end{array}$ \\
\hline
\end{tabular}

\section{Analysis 20.2. Comparison 20: Laser ALA-PDT vs broadband light ALA-PDT, Outcome 2: Pain}

\begin{tabular}{|c|c|c|c|c|c|c|}
\hline & \multicolumn{2}{|c|}{ Laser } & \multicolumn{2}{|c|}{ Broad band } & Risk Ratio & Risk Ratio \\
\hline Study or Subgroup & Events & Total & Events & Total & M-H, Random, $95 \%$ CI & M-H, Random, 95\% CI \\
\hline
\end{tabular}

20.2.1 Discomfort during/after illumination

$\begin{array}{llllll}\text { Soler } 2000 & 34 & 41 & 32 & 42 & 1.09[0.87,1.35]\end{array}$

20.2.2 Discomfort during 1st week after treatment

$\begin{array}{llllll}\text { Soler } 2000 & 28 & 41 & 31 & 42 & 0.93[0.70,1.22]\end{array}$

$\begin{array}{lllll}28 & 41 & 31 & 42 & 0.93[0.70,1.22]\end{array}$

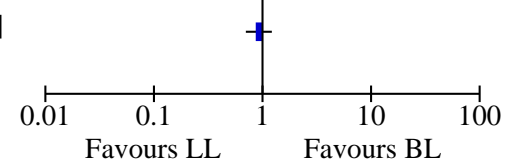

Comparison 21. AFXL MAL-PDT vs MAL-PDT

\begin{tabular}{lllll}
\hline Outcome or subgroup title & $\begin{array}{l}\text { No. of } \\
\text { studies }\end{array}$ & $\begin{array}{l}\text { No. of } \\
\text { partici- } \\
\text { pants }\end{array}$ & Statistical method & Effect size \\
\hline $\begin{array}{l}\text { 21.1 Cosmetic outcome (good/excel- } \\
\text { lent) }\end{array}$ & 2 & & Risk Ratio (M-H, Random, 95\% Cl) & Subtotals only \\
\hline 21.1 .1 Observer rated at 1 year & 2 & 57 & Risk Ratio (M-H, Random, 95\% Cl) & $1.12[0.54,2.31]$ \\
\hline 21.1 .2 Participant rated at 1 year & 1 & 23 & Risk Ratio (M-H, Random, 95\% Cl) & $0.94[0.75,1.17]$ \\
\hline
\end{tabular}




\begin{tabular}{lllll}
\hline Outcome or subgroup title & $\begin{array}{l}\text { No. of } \\
\text { studies }\end{array}$ & $\begin{array}{l}\text { No. of } \\
\text { partici- } \\
\text { pants }\end{array}$ & Statistical method & Effect size \\
\hline 21.2 Early treatment failure & 2 & 71 & Risk Ratio (M-H, Random, 95\% Cl) & $0.38[0.17,0.84]$ \\
\hline
\end{tabular}

Analysis 21.1. Comparison 21: AFXL MAL-PDT vs MAL-PDT, Outcome 1: Cosmetic outcome (good/excellent)

\begin{tabular}{|c|c|c|c|c|c|}
\hline & AFXL MAL-PDT & MAL-PDT & & Risk Ratio & Risk Ratio \\
\hline Study or Subgroup & Events & Events & Weight & M-H, Random, $95 \%$ CI & M-H, Random, $95 \%$ CI \\
\hline
\end{tabular}

\begin{tabular}{lrrrrrr}
\hline 21.1.1 Observer rated at 1 year & & & & & & \\
Choi 2016 & 17 & 18 & 16 & 16 & $65.8 \%$ & $0.95[0.81,1.11]$ \\
Haak 2015 & 8 & 13 & 4 & 10 & $34.2 \%$ & $1.54[0.64,3.68]$ \\
Subtotal (95\% CI) & & $\mathbf{3 1}$ & & $\mathbf{2 6}$ & $\mathbf{1 0 0 . 0 \%}$ & $\mathbf{1 . 1 2}[\mathbf{0 . 5 4 , 2 . 3 1}]$ \\
Total events: & 25 & & 20 & & &
\end{tabular}

Heterogeneity: $\mathrm{Tau}^{2}=0.20 ; \mathrm{Chi}^{2}=2.97, \mathrm{df}=1(\mathrm{P}=0.08) ; \mathrm{I}^{2}=66 \%$

Test for overall effect: $\mathrm{Z}=0.31(\mathrm{P}=0.76)$

21.1.2 Participant rated at 1 year

Haak 201512

Subtotal (95\% CI)

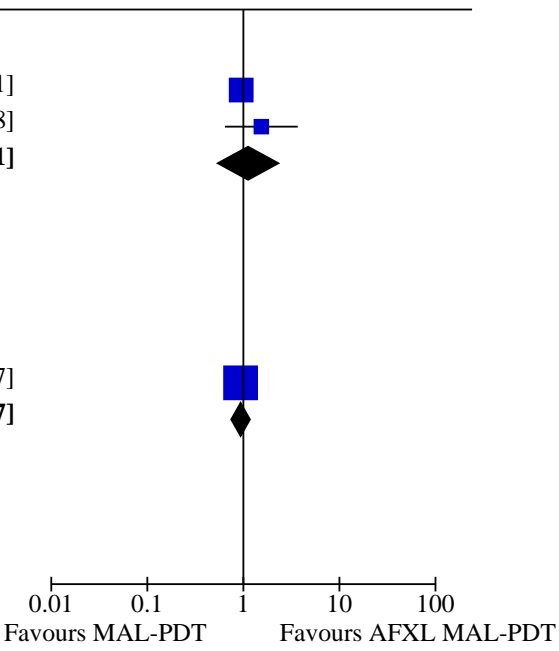

\section{Analysis 21.2. Comparison 21: AFXL MAL-PDT vs MAL-PDT, Outcome 2: Early treatment failure}

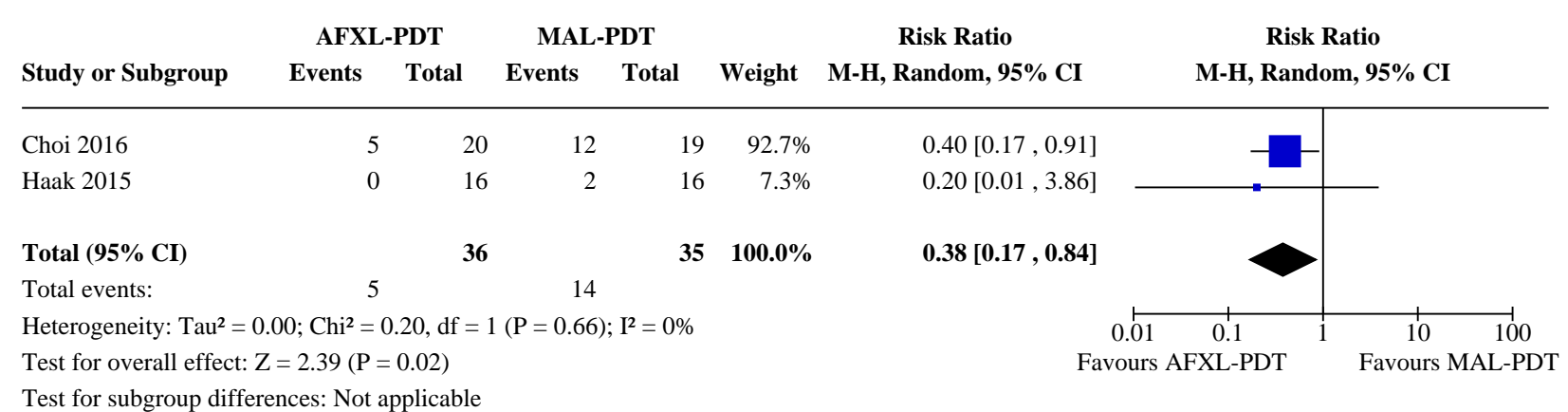

\section{Comparison 22. MAL-PDT vs placebo}

\begin{tabular}{lllll}
\hline Outcome or subgroup title & $\begin{array}{l}\text { No. of } \\
\text { studies }\end{array}$ & $\begin{array}{l}\text { No. of } \\
\text { partici- } \\
\text { pants }\end{array}$ & Statistical method & Effect size \\
\hline $\begin{array}{l}22.1 \text { Cosmetic outcome (good/excellent, ob- } \\
\text { server rated at } 6 \text { months)) }\end{array}$ & 1 & Risk Ratio (M-H, Random, 95\% Cl) & $\begin{array}{l}\text { Totals not select- } \\
\text { ed }\end{array}$ \\
\hline
\end{tabular}




\begin{tabular}{lllll}
\hline Outcome or subgroup title & $\begin{array}{l}\text { No. of } \\
\text { studies }\end{array}$ & $\begin{array}{l}\text { No. of } \\
\text { partici- } \\
\text { pants }\end{array}$ & Statistical method & Effect size \\
\hline 22.2 Pain (frequency reported as local AE) & 1 & & Risk Ratio (M-H, Random, 95\% Cl) & $\begin{array}{l}\text { Totals not select- } \\
\text { ed }\end{array}$ \\
\hline 22.3 Early treatment failure (lesions) & 1 & Risk Ratio (M-H, Random, 95\% Cl) & $\begin{array}{l}\text { Totals not select- } \\
\text { ed }\end{array}$ \\
\hline
\end{tabular}

Analysis 22.1. Comparison 22: MAL-PDT vs placebo, Outcome 1:

Cosmetic outcome (good/excellent, observer rated at 6 months))

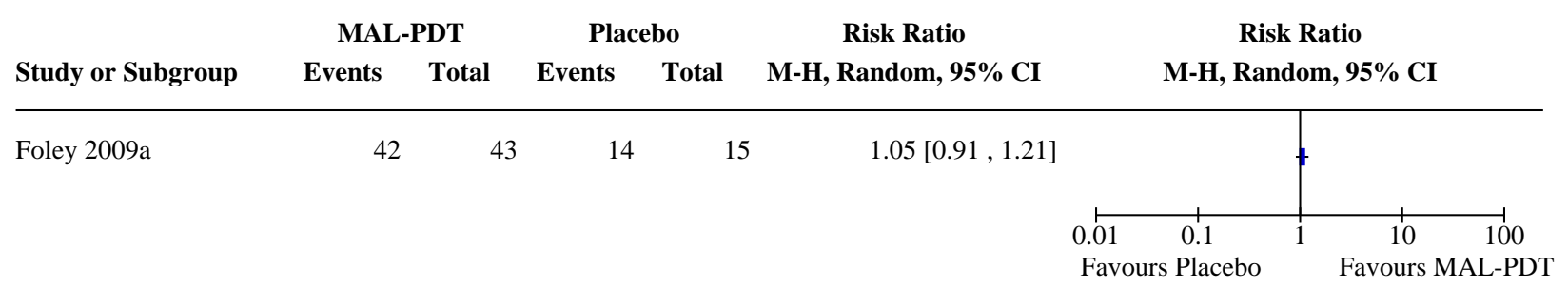

Analysis 22.2. Comparison 22: MAL-PDT vs placebo, Outcome 2: Pain (frequency reported as local AE)

\begin{tabular}{|c|c|c|c|c|c|c|}
\hline & MA & PDT & Pla & & Risk Ratio & Risk Ratio \\
\hline Study or Subgroup & Events & Total & Events & Total & M-H, Random, 95\% CI & M-H, Random, 95\% CI \\
\hline
\end{tabular}

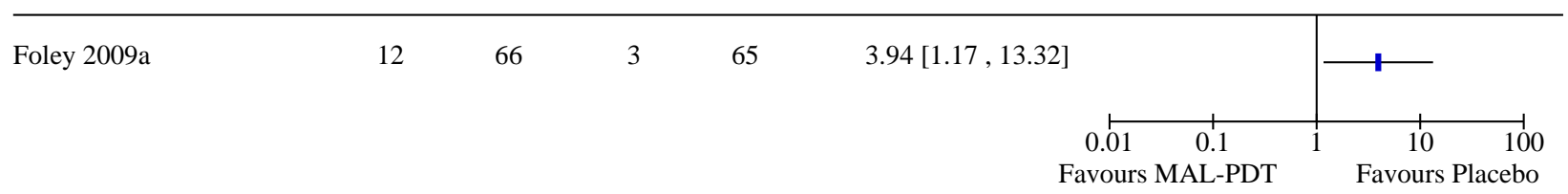

Analysis 22.3. Comparison 22: MAL-PDT vs placebo, Outcome 3: Early treatment failure (lesions)

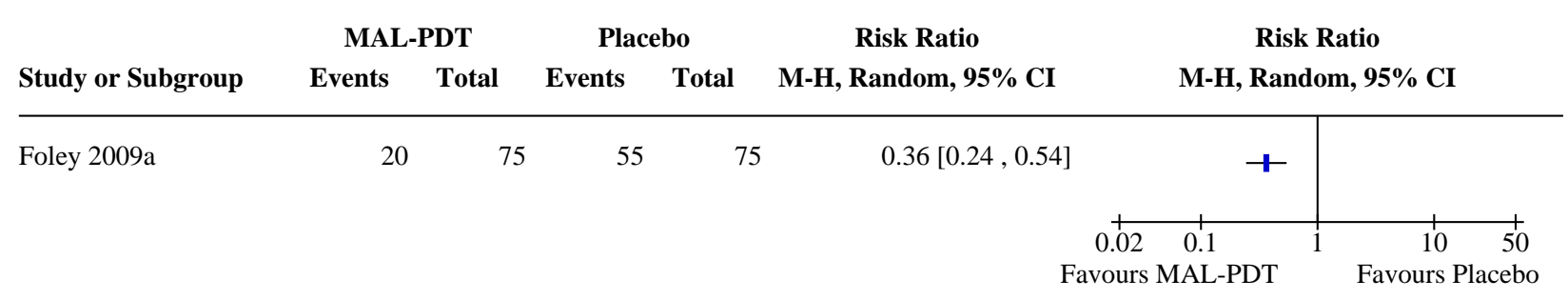

Comparison 23. Imiquimod cream vs 5-FU cream

\begin{tabular}{lllll}
\hline Outcome or subgroup title & $\begin{array}{l}\text { No. of } \\
\text { studies }\end{array}$ & $\begin{array}{l}\text { No. of } \\
\text { partici- } \\
\text { pants }\end{array}$ & Statistical method & Effect size \\
\hline 23.1 Recurrence at 3 years & 1 & Risk Ratio (M-H, Random, 95\% Cl) & Totals not selected \\
\hline
\end{tabular}




\begin{tabular}{lllll}
\hline Outcome or subgroup title & $\begin{array}{l}\text { No. of } \\
\text { studies }\end{array}$ & $\begin{array}{l}\text { No. of } \\
\text { partici- } \\
\text { pants }\end{array}$ & Statistical method & Effect size \\
\hline 23.2 Recurrence at 5 years & 1 & Risk Ratio (M-H, Random, 95\% Cl) & Totals not selected \\
\hline $\begin{array}{l}\text { 23.3 Cosmetic outcome (good/ex- } \\
\text { cellent, observer rated at 1 year) }\end{array}$ & 1 & Risk Ratio (M-H, Random, 95\% Cl) & Totals not selected \\
\hline 23.4 Pain (moderate/severe) & 1 & Risk Ratio (M-H, Random, 95\% Cl) & Totals not selected \\
\hline 23.5 Early treatment failure & 1 & Risk Ratio (M-H, Random, 95\% Cl) & Totals not selected \\
\hline
\end{tabular}

Analysis 23.1. Comparison 23: Imiquimod cream vs 5-FU cream, Outcome 1: Recurrence at 3 years

\begin{tabular}{|c|c|c|c|c|c|c|c|}
\hline \multirow[b]{2}{*}{ Study or Subgroup } & \multicolumn{2}{|c|}{ Imiquimod cream } & \multicolumn{2}{|c|}{ 5-FU cream } & \multirow{2}{*}{$\begin{array}{c}\text { Risk Ratio } \\
\text { M-H, Random, 95\% CI }\end{array}$} & \multirow{2}{*}{\multicolumn{2}{|c|}{$\begin{array}{c}\text { Risk Ratio } \\
\text { M-H, Random, 95\% CI }\end{array}$}} \\
\hline & Events & Total & Events & Total & & & \\
\hline Arits 2013 & 34 & 145 & 50 & 146 & $0.68[0.47,0.99]$ & 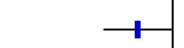 & \\
\hline & & & & & Favours & $\begin{array}{lc}0.2 & 0.5 \\
\text { limod cream }\end{array}$ & $\begin{array}{c}2 \\
\text { Favours } 5\end{array}$ \\
\hline
\end{tabular}

Analysis 23.2. Comparison 23: Imiquimod cream vs 5-FU cream, Outcome 2: Recurrence at 5 years

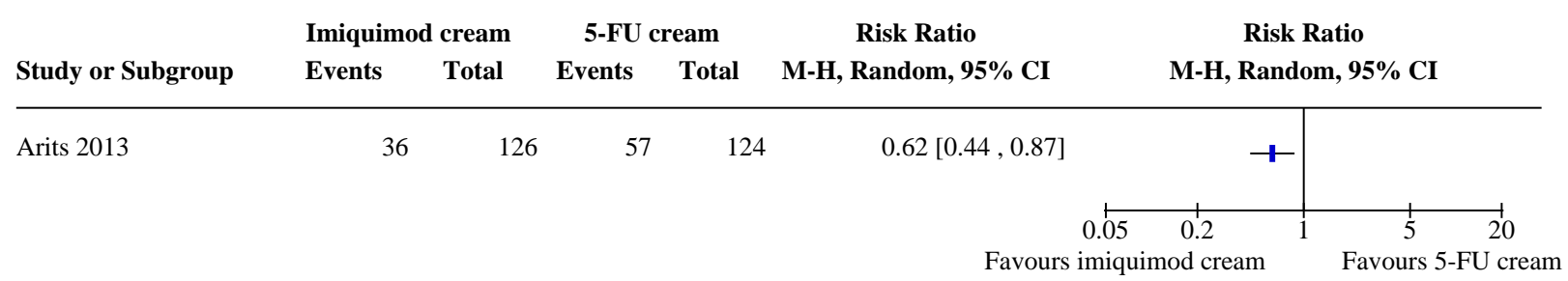

Analysis 23.3. Comparison 23: Imiquimod cream vs 5-FU cream, Outcome 3: Cosmetic outcome (good/excellent, observer rated at 1 year)

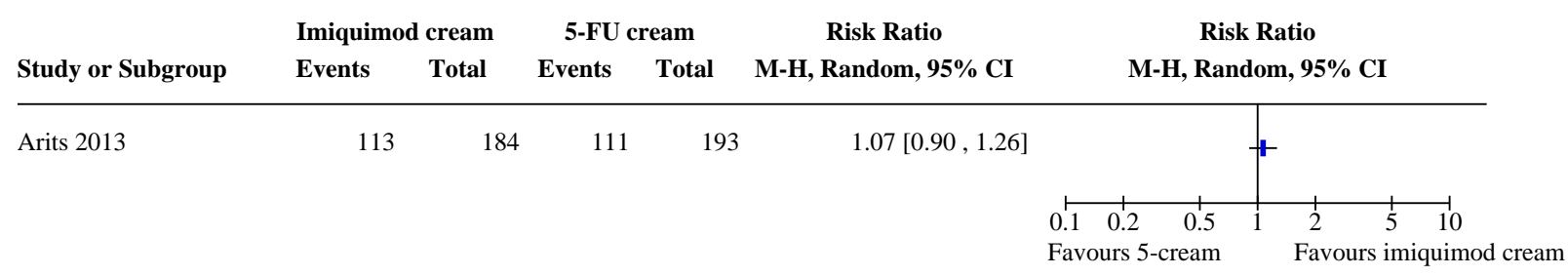


Analysis 23.4. Comparison 23: Imiquimod cream vs 5-FU cream, Outcome 4: Pain (moderate/severe)

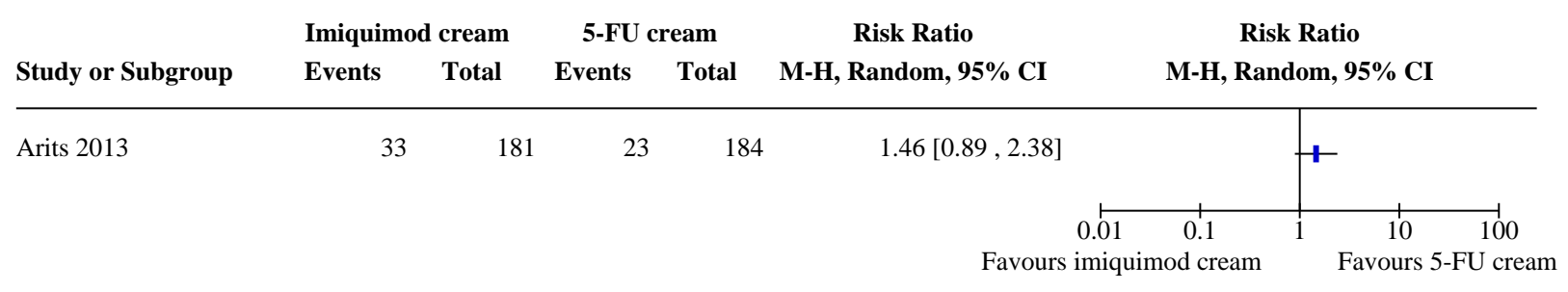

Analysis 23.5. Comparison 23: Imiquimod cream vs 5-FU cream, Outcome 5: Early treatment failure

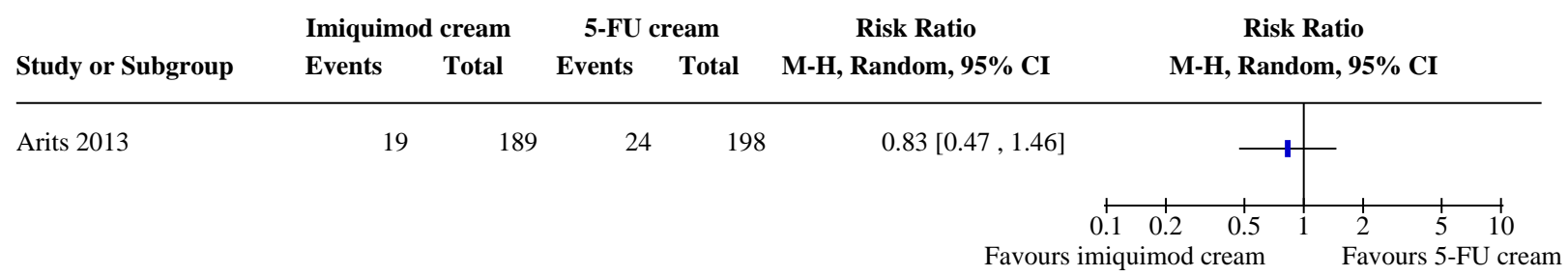

Comparison 24. Imiquimod vs radiotherapy

\begin{tabular}{lllll}
\hline Outcome or subgroup title & $\begin{array}{l}\text { No. of } \\
\text { studies }\end{array}$ & $\begin{array}{l}\text { No. of } \\
\text { partici- } \\
\text { pants }\end{array}$ & Statistical method & Effect size \\
\hline $\begin{array}{l}24.1 \text { Cosmetic outcome (excellent, participant rated } \\
\text { at } 6 \text { weeks) }\end{array}$ & 1 & & $\begin{array}{l}\text { Risk Ratio (M-H, Random, } \\
95 \% \mathrm{Cl})\end{array}$ & $\begin{array}{l}\text { Totals not se- } \\
\text { lected }\end{array}$ \\
\hline
\end{tabular}

Analysis 24.1. Comparison 24: Imiquimod vs radiotherapy, Outcome 1: Cosmetic outcome (excellent, participant rated at 6 weeks)

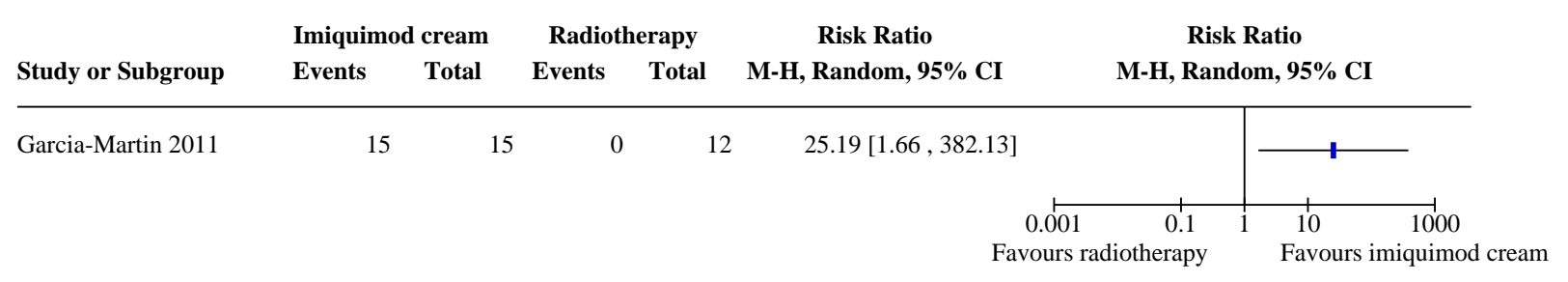

Comparison 25. Imiquimod cream under occlusion vs no occlusion

\begin{tabular}{lllll}
\hline Outcome or subgroup title & $\begin{array}{l}\text { No. of } \\
\text { studies }\end{array}$ & $\begin{array}{l}\text { No. of } \\
\text { partici- } \\
\text { pants }\end{array}$ & Statistical method & Effect size \\
\hline 25.1 Early treatment failure & 2 & & Risk Ratio $(\mathrm{M}-\mathrm{H}$, Random, 95\% Cl) & Totals not selected \\
\hline $25.1 .1 \mathrm{sBCC}$ & 1 & Risk Ratio $(\mathrm{M}-\mathrm{H}$, Random, 95\% Cl) & Totals not selected \\
\hline
\end{tabular}




\begin{tabular}{lllll}
\hline Outcome or subgroup title & $\begin{array}{l}\text { No. of } \\
\text { studies }\end{array}$ & $\begin{array}{l}\text { No. of } \\
\text { partici- } \\
\text { pants }\end{array}$ & Statistical method & Effect size \\
\hline $25.1 .2 \mathrm{nBCC}$ & 1 & & Risk Ratio (M-H, Random, $95 \% \mathrm{Cl})$ & Totals not selected \\
\hline
\end{tabular}

Analysis 25.1. Comparison 25: Imiquimod cream under occlusion vs no occlusion, Outcome 1: Early treatment failure

\begin{tabular}{|c|c|c|c|c|c|c|}
\hline \multirow[b]{2}{*}{ Study or Subgroup } & \multicolumn{2}{|c|}{ With occlusion } & \multicolumn{2}{|c|}{ Without occlusion } & \multirow{2}{*}{$\begin{array}{c}\text { Risk Ratio } \\
\text { M-H, Random, 95\% CI }\end{array}$} & \multirow{2}{*}{$\begin{array}{c}\text { Risk Ratio } \\
\text { M-H, Random, 95\% CI }\end{array}$} \\
\hline & Events & Total & Events & Total & & \\
\hline
\end{tabular}

\begin{tabular}{|c|c|c|c|c|c|}
\hline \multicolumn{6}{|l|}{ 25.1.1 sBCC } \\
\hline Sterry $2002 a$ & 15 & 44 & 18 & 49 & $0.93[0.53,1.61]$ \\
\hline \multicolumn{6}{|l|}{ 25.1.2 nBCC } \\
\hline Sterry $2002 b$ & 19 & 45 & 21 & 45 & $0.90[0.57,1.44]$ \\
\hline
\end{tabular}

Sterry $2002 b$

$19 \quad 21 \quad 45$

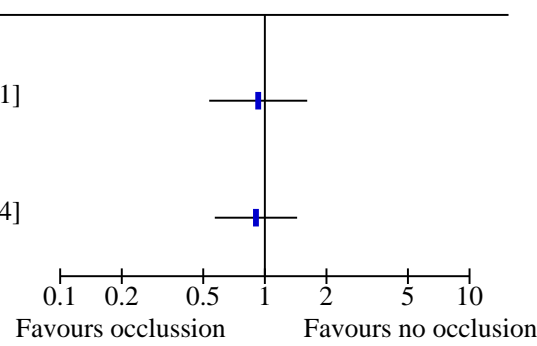

\section{Comparison 26. Imiquimod cream vs vehicle}

\begin{tabular}{lllll}
\hline Outcome or subgroup title & $\begin{array}{l}\text { No. of } \\
\text { studies }\end{array}$ & $\begin{array}{l}\text { No. of partici- } \\
\text { pants }\end{array}$ & Statistical method & Effect size \\
\hline 26.1 Early treatment failure & 5 & 1145 & Risk Ratio (M-H, Random, 95\% Cl) & $0.25[0.19,0.32]$ \\
\hline
\end{tabular}

Analysis 26.1. Comparison 26: Imiquimod cream vs vehicle, Outcome 1: Early treatment failure

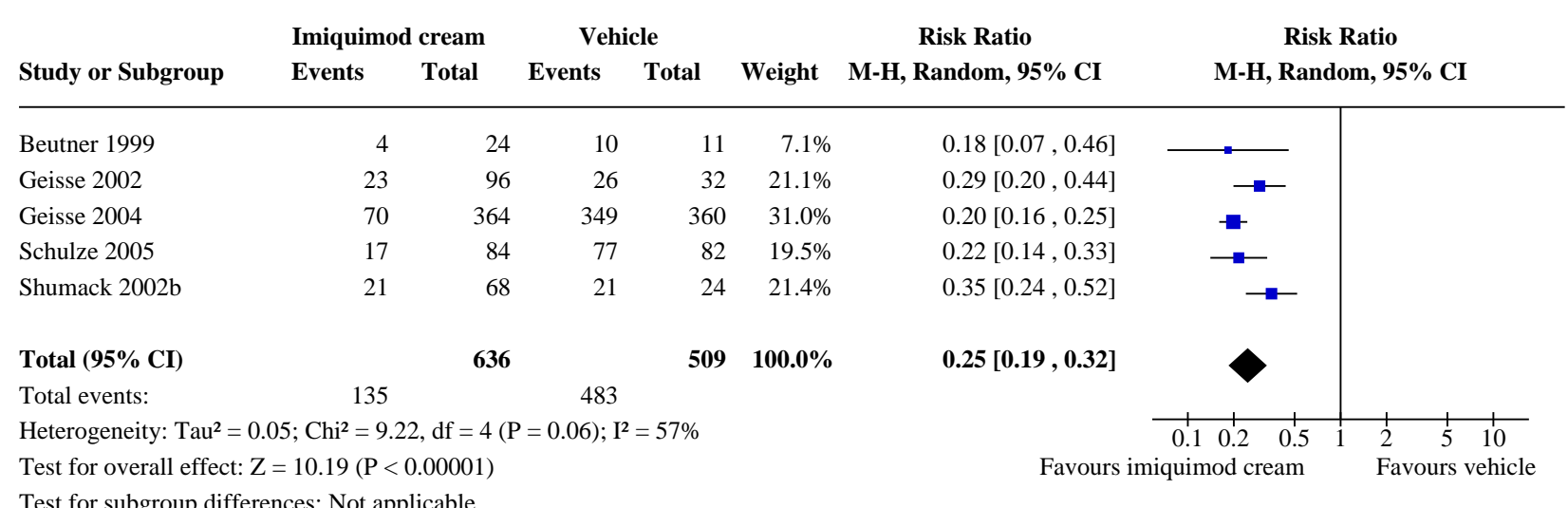


Comparison 27. Imiquimod 5 weeks vs 8 weeks

\begin{tabular}{llll}
\hline Outcome or subgroup title & $\begin{array}{l}\text { No. of } \\
\text { studies }\end{array}$ & $\begin{array}{l}\text { No. of par- } \\
\text { ticipants }\end{array}$ & Statistical method \\
\hline 27.1 Recurrence at 1 year & 1 & Risk Ratio (M-H, Random, 95\% Cl) & Totals not selected \\
\hline
\end{tabular}

Analysis 27.1. Comparison 27: Imiquimod 5 weeks vs 8 weeks, Outcome 1: Recurrence at 1 year

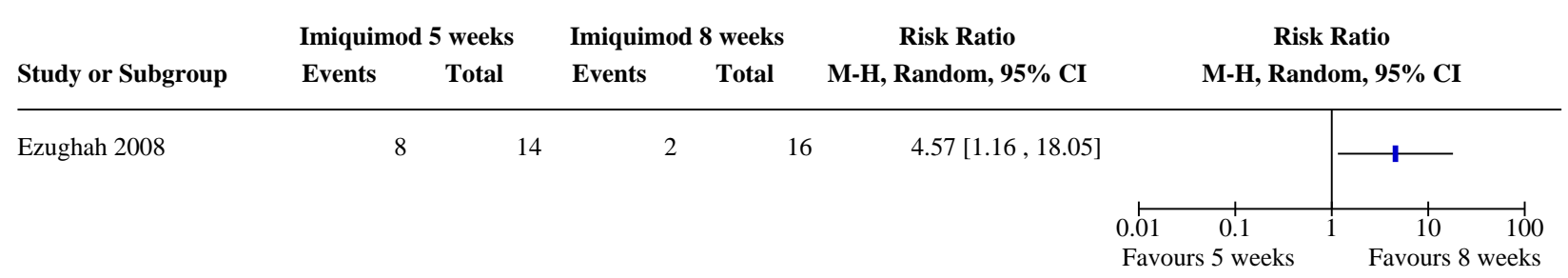

Comparison 28. Imiquimod 8 weeks vs 12 weeks

\begin{tabular}{lllll}
\hline Outcome or subgroup title & $\begin{array}{l}\text { No. of } \\
\text { studies }\end{array}$ & $\begin{array}{l}\text { No. of par- } \\
\text { ticipants }\end{array}$ & Statistical method & Effect size \\
\hline 28.1 Early treatment failure & 1 & Risk Ratio (M-H, Random, 95\% Cl) & Totals not selected \\
\hline
\end{tabular}

Analysis 28.1. Comparison 28: Imiquimod 8 weeks vs 12 weeks, Outcome 1: Early treatment failure

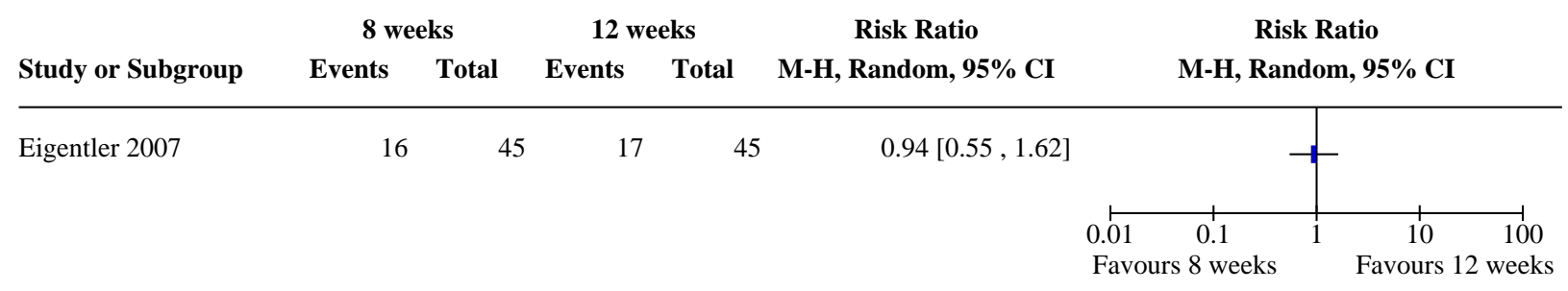

Comparison 29. 5-FU in PC vs 5-FU in petrolatum

\begin{tabular}{lllll}
\hline Outcome or subgroup title & $\begin{array}{l}\text { No. of } \\
\text { studies }\end{array}$ & $\begin{array}{l}\text { No. of par- } \\
\text { ticipants }\end{array}$ & Statistical method & Effect size \\
\hline 29.1 Early treatment failure (lesions) & 1 & Risk Ratio (M-H, Random, 95\% Cl) & Subtotals only \\
\hline
\end{tabular}


Analysis 29.1. Comparison 29: 5-FU in PC vs 5-FU in petrolatum, Outcome 1: Early treatment failure (lesions)

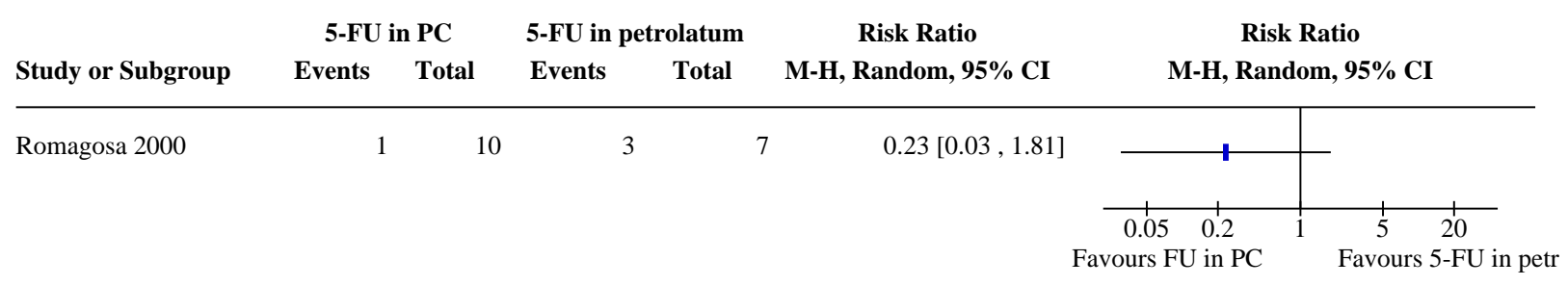

Comparison 30. 5-FU/epinephrine gel (1.0 mL once a week for 6 weeks vs $0.5 \mathrm{~mL}$ once a week for 6 weeks

\begin{tabular}{lllll}
\hline Outcome or subgroup title & $\begin{array}{l}\text { No. of } \\
\text { studies }\end{array}$ & $\begin{array}{l}\text { No. of par- } \\
\text { ticipants }\end{array}$ & Statistical method & Effect size \\
\hline 30.1 Early treatment failure & 1 & Risk Ratio (M-H, Random, 95\% Cl) & Subtotals only \\
\hline
\end{tabular}

Analysis 30.1. Comparison 30: 5-FU/epinephrine gel (1.0 mL once a week for 6 weeks vs $0.5 \mathrm{~mL}$ once a week for 6 weeks, Outcome 1: Early treatment failure

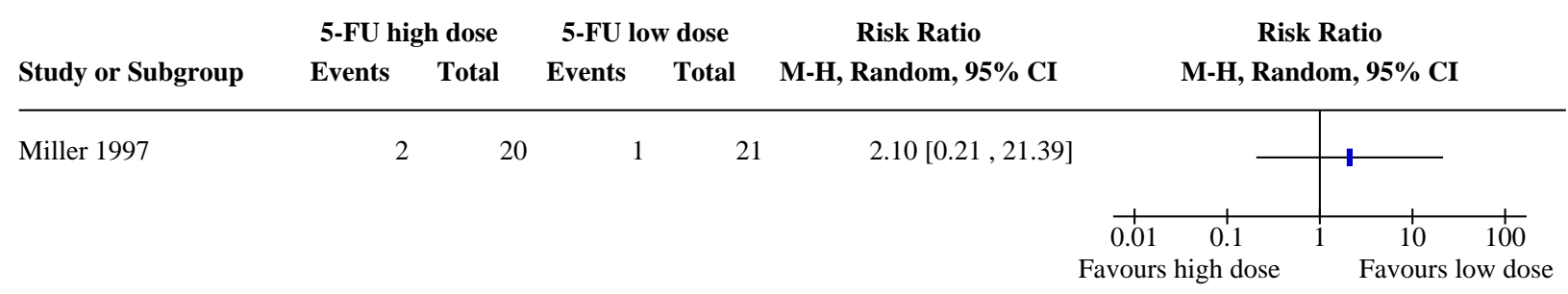

Comparison 31. 5-FU/epinephrine gel(1.0 mL twice a week for 3 weeks vs $0.5 \mathrm{~mL}$ twice a week for 3 weeks)

\begin{tabular}{lllll}
\hline Outcome or subgroup title & $\begin{array}{l}\text { No. of } \\
\text { studies }\end{array}$ & $\begin{array}{l}\text { No. of par- } \\
\text { ticipants }\end{array}$ & Statistical method & Effect size \\
\hline 31.1 Early treatment failure & 1 & & Risk Ratio (M-H, Random, 95\% Cl) & Subtotals only \\
\hline
\end{tabular}

Analysis 31.1. Comparison 31: 5-FU/epinephrine gel(1.0 mL twice a week for 3 weeks vs $0.5 \mathrm{~mL}$ twice a week for 3 weeks), Outcome 1: Early treatment failure

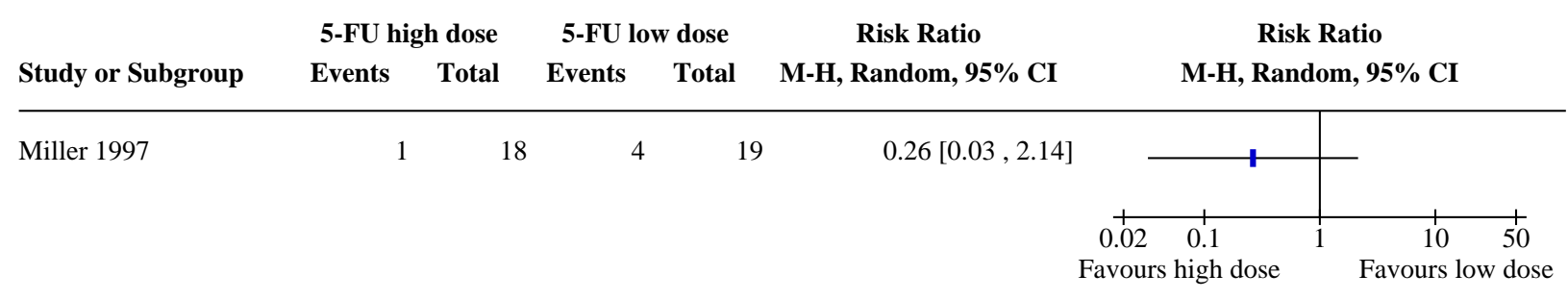


Comparison 32. 5-FU/epinephrine gel( $0.5 \mathrm{ml}$ twice a week for 4 weeks vs $0.5 \mathrm{~mL}$ three times a week for 2 weeks.

\begin{tabular}{lllll}
\hline Outcome or subgroup title & $\begin{array}{l}\text { No. of } \\
\text { studies }\end{array}$ & $\begin{array}{l}\text { No. of par- } \\
\text { ticipants }\end{array}$ & Statistical method & Effect size \\
\hline 32.1 Early treatment failure & 1 & Risk Ratio (M-H, Random, 95\% Cl) & Subtotals only \\
\hline
\end{tabular}

Analysis 32.1. Comparison 32: 5-FU/epinephrine gel( $0.5 \mathrm{ml}$ twice a week for 4 weeks vs $0.5 \mathrm{~mL}$ three times a week for 2 weeks., Outcome 1: Early treatment failure

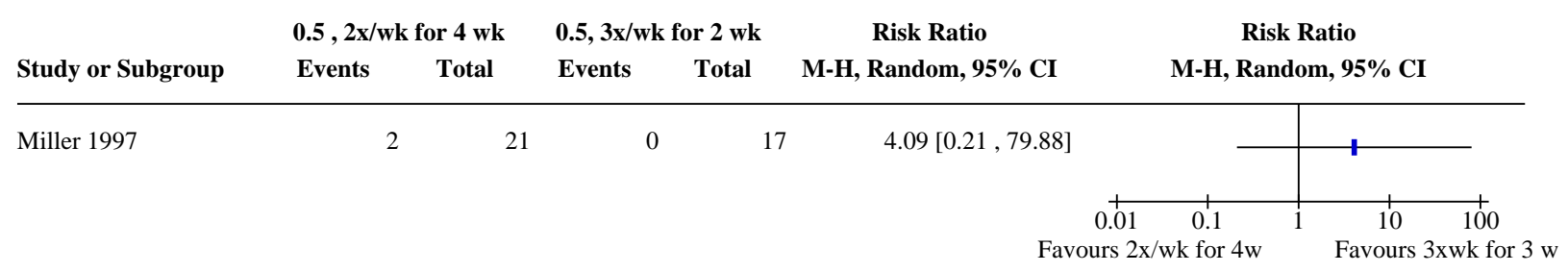

Comparison 33. Electrochemotherapy vs surgical excision

\begin{tabular}{lllll}
\hline Outcome or subgroup title & $\begin{array}{l}\text { No. of } \\
\text { studies }\end{array}$ & $\begin{array}{l}\text { No. of } \\
\text { partici- } \\
\text { pants }\end{array}$ & Statistical method & Effect size \\
\hline 33.1 Recurrence at 3 years & 1 & Risk Ratio (M-H, Random, 95\% Cl) & Totals not selected \\
\hline 33.2 Recurrence at 5 years & 1 & Risk Ratio (M-H, Random, 95\% Cl) & Totals not selected \\
\hline
\end{tabular}

Analysis 33.1. Comparison 33: Electrochemotherapy vs surgical excision, Outcome 1: Recurrence at 3 years

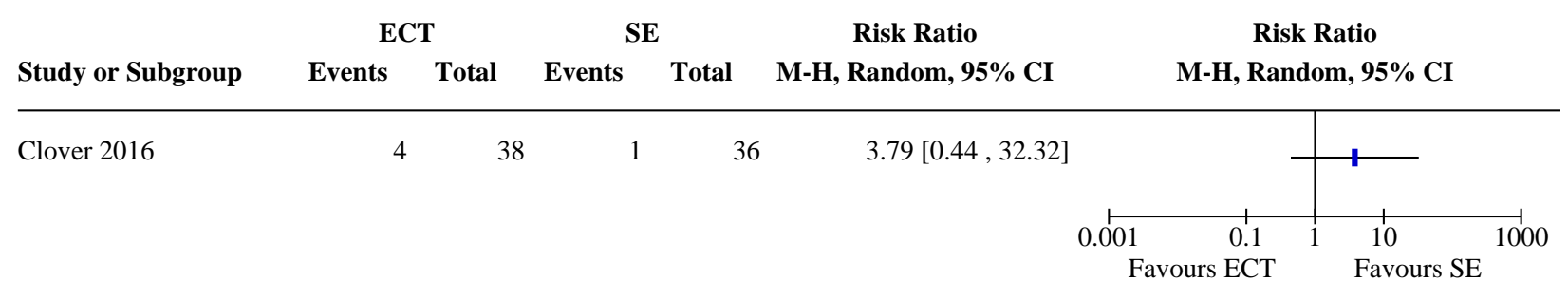

Analysis 33.2. Comparison 33: Electrochemotherapy vs surgical excision, Outcome 2: Recurrence at 5 years

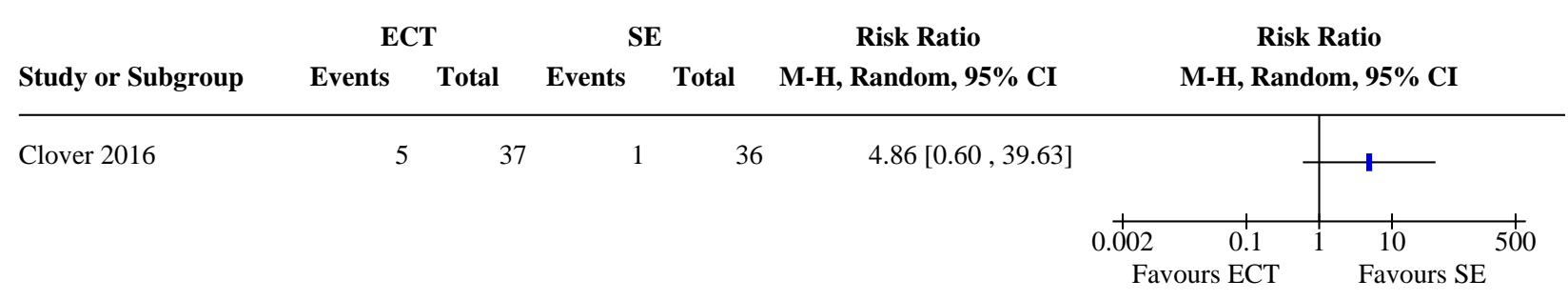


Comparison 34. Ingenol vs vehicle (day 1+2)

\begin{tabular}{|c|c|c|c|c|}
\hline Outcome or subgroup title & $\begin{array}{l}\text { No. of } \\
\text { studies }\end{array}$ & $\begin{array}{l}\text { No. of } \\
\text { partici- } \\
\text { pants }\end{array}$ & Statistical method & Effect size \\
\hline 34.1 Early treatment failure & 1 & & Risk Ratio (M-H, Random, 95\% Cl) & Totals not selected \\
\hline 34.1 .1 Ingenol $0.05 \%$ vs vehicle & 1 & & Risk Ratio (M-H, Random, 95\% Cl) & Totals not selected \\
\hline 34.1 .2 Ingenol $0.01 \%$ vs vehicle & 1 & & Risk Ratio (M-H, Random, 95\% Cl) & Totals not selected \\
\hline 34.1.3 Ingenol $0.0025 \%$ vs vehicle & 1 & & Risk Ratio (M-H, Random, 95\% Cl) & Totals not selected \\
\hline
\end{tabular}

Analysis 34.1. Comparison 34: Ingenol vs vehicle (day 1+2), Outcome 1: Early treatment failure

\begin{tabular}{|c|c|c|c|c|c|c|}
\hline & \multicolumn{2}{|c|}{ Ingenol } & \multicolumn{2}{|c|}{ Vehicle } & Risk Ratio & Risk Ratio \\
\hline Study or Subgroup & Events & Total & Events & Total & M-H, Random, $95 \%$ CI & M-H, Random, $95 \%$ CI \\
\hline
\end{tabular}

34.1.1 Ingenol $0.05 \%$ vs vehicle

Siller 2010

3

34.1.2 Ingenol 0.01\% vs vehicle

Siller 2010

6

8

$6 \quad 6$

$0.78[0.49,1.23]$

34.1.3 Ingenol $0.0025 \%$ vs vehicle

Siller 2010

8

$8 \quad 6 \quad 6$

$1.00[0.77,1.30]$

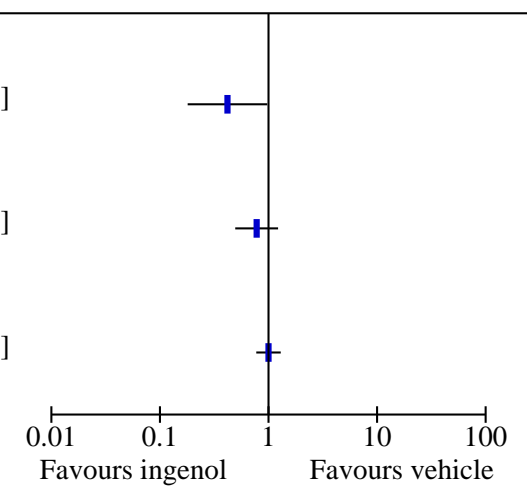

Comparison 35. Ingenol vs vehicle (day 1+8)

\begin{tabular}{lllll}
\hline Outcome or subgroup title & $\begin{array}{l}\text { No. of } \\
\text { studies }\end{array}$ & $\begin{array}{l}\text { No. of } \\
\text { partici- } \\
\text { pants }\end{array}$ & Statistical method & Effect size \\
\hline 35.1 Early treatment failure & 1 & Risk Ratio $(\mathrm{M}-\mathrm{H}$, Random, 95\% Cl) & Totals not selected \\
\hline 35.1 .1 Ingenol 0.05\% vs vehicle & 1 & Risk Ratio (M-H, Random, 95\% Cl) & Totals not selected \\
\hline 35.1 .2 Ingenol 0.01\% vs vehicle & 1 & Risk Ratio (M-H, Random, 95\% Cl) & Totals not selected \\
\hline 35.1 .3 Ingenol 0.0025\% vs vehicle & 1 & Risk Ratio (M-H, Random, 95\% Cl) & Totals not selected \\
\hline
\end{tabular}


Analysis 35.1. Comparison 35: Ingenol vs vehicle (day 1+8), Outcome 1: Early treatment failure

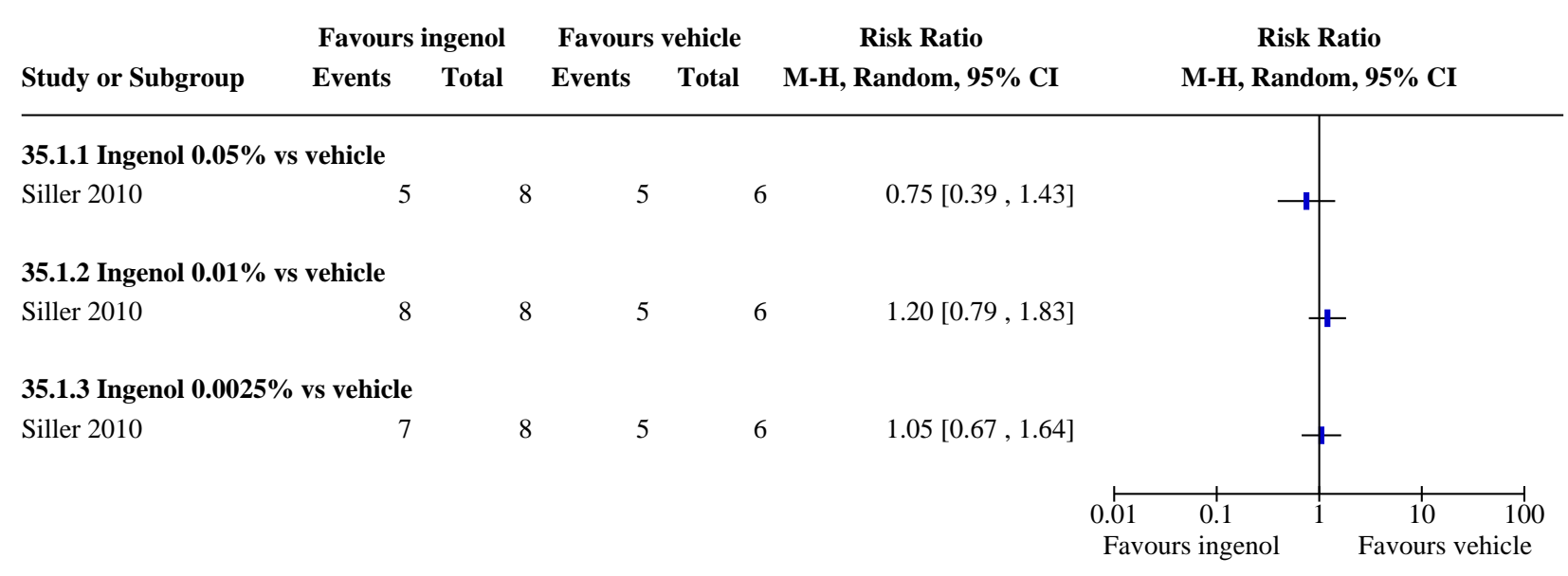

Comparison 36. Ingenol under occlusion vs no occlusion

\begin{tabular}{lllll}
\hline Outcome or subgroup title & $\begin{array}{l}\text { No. of } \\
\text { studies }\end{array}$ & $\begin{array}{l}\text { No. of } \\
\text { partici- } \\
\text { pants }\end{array}$ & Statistical method & Effect size \\
\hline 36.1 Early treatment failure & 1 & Risk Ratio (M-H, Random, 95\% Cl) & Totals not selected \\
\hline 36.1 .1 Full occlusion vs no occlusion & 1 & Risk Ratio (M-H, Random, 95\% Cl) & Totals not selected \\
\hline 36.1 .2 Semi-occlusion vs no occlusion & 1 & Risk Ratio (M-H, Random, 95\% Cl) & Totals not selected \\
\hline
\end{tabular}

Analysis 36.1. Comparison 36: Ingenol under occlusion vs no occlusion, Outcome 1: Early treatment failure

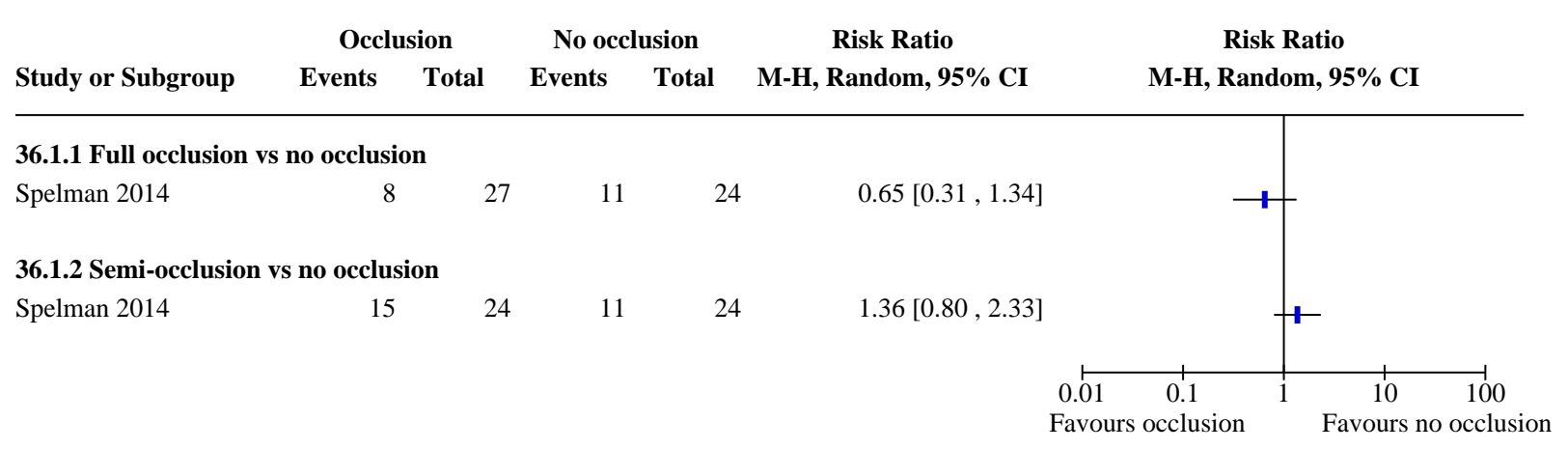

Comparison 37. Solasodine glycoside cream vs vehicle

\begin{tabular}{lllll}
\hline Outcome or subgroup title & $\begin{array}{l}\text { No. of } \\
\text { studies }\end{array}$ & $\begin{array}{l}\text { No. of par- } \\
\text { ticipants }\end{array}$ & Statistical method & Effect size \\
\hline 37.1 Early treatment failure & 1 & Risk Ratio (M-H, Random, 95\% Cl) & Subtotals only \\
\hline
\end{tabular}


Analysis 37.1. Comparison 37: Solasodine glycoside cream vs vehicle, Outcome 1: Early treatment failure

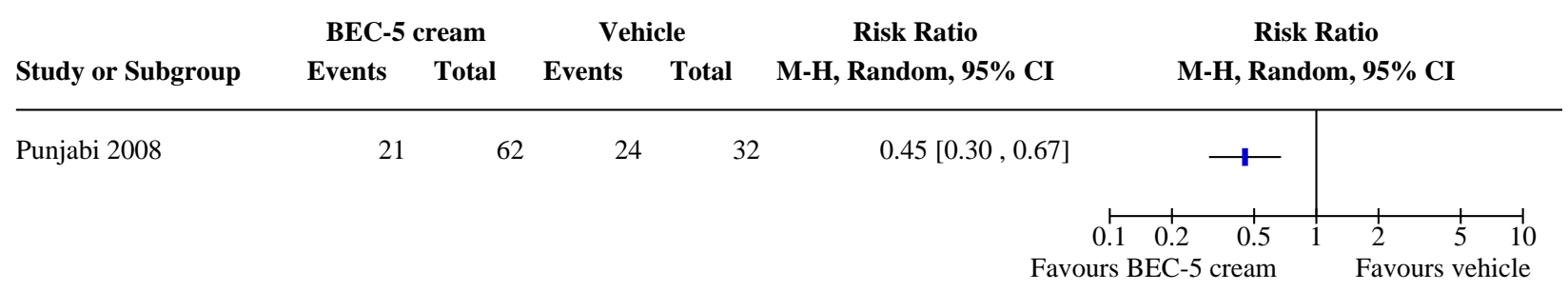

Comparison 38. Valproic acid gel + Tazarotene gel vs placebo + Tazarotene

\begin{tabular}{lllll}
\hline Outcome or subgroup title & $\begin{array}{l}\text { No. of } \\
\text { studies }\end{array}$ & $\begin{array}{l}\text { No. of par- } \\
\text { ticipants }\end{array}$ & Statistical method & Effect size \\
\hline 38.1 Early treatment failure & 1 & Risk Ratio (M-H, Random, 95\% Cl) & Totals not selected \\
\hline
\end{tabular}

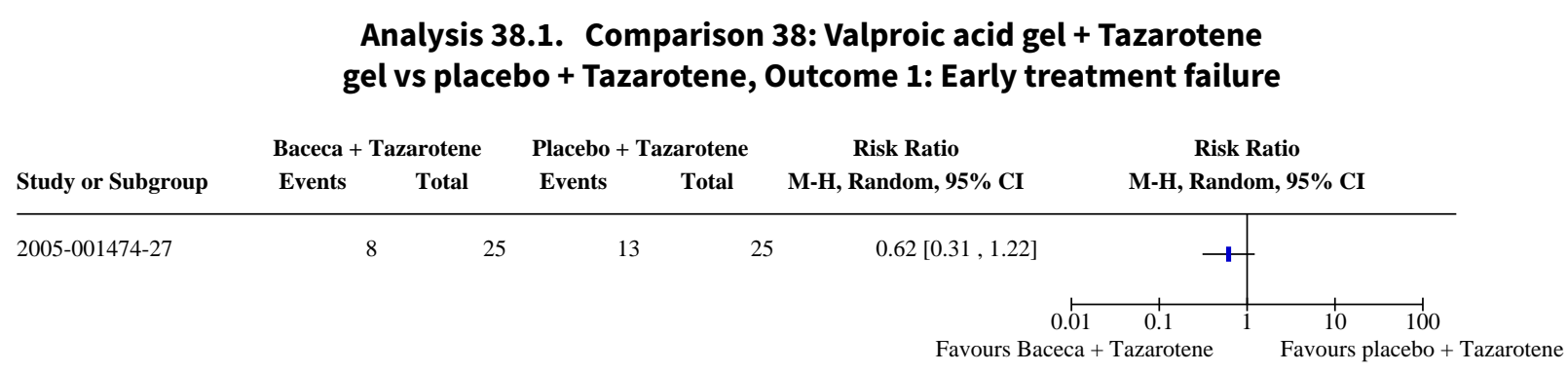

\section{Comparison 39. Diclofenac gel vs calcitriol ointment}

\begin{tabular}{lllll}
\hline Outcome or subgroup title & $\begin{array}{l}\text { No. of } \\
\text { studies }\end{array}$ & $\begin{array}{l}\text { No. of par- } \\
\text { ticipants }\end{array}$ & Statistical method & Effect size \\
\hline 39.1 Early treatment failure & 1 & Risk Ratio (M-H, Random, 95\% Cl) & Totals not selected \\
\hline
\end{tabular}

Analysis 39.1. Comparison 39: Diclofenac gel vs calcitriol ointment, Outcome 1: Early treatment failure

\begin{tabular}{|c|c|c|c|c|c|c|c|}
\hline \multirow[b]{2}{*}{ Study or Subgroup } & \multicolumn{2}{|c|}{ Diclofenac gel } & \multicolumn{2}{|c|}{ Calcitriol oint } & \multirow{2}{*}{$\begin{array}{c}\text { Risk Ratio } \\
\text { M-H, Random, 95\% CI }\end{array}$} & \multirow{2}{*}{\multicolumn{2}{|c|}{$\begin{array}{c}\text { Risk Ratio } \\
\text { M-H, Random, 95\% CI }\end{array}$}} \\
\hline & Events & Total & Events & Total & & & \\
\hline \multirow[t]{2}{*}{ Brinkhuizen 2016} & 16 & 30 & 31 & 32 & $0.55[0.39,0.77]$ & 1 & \\
\hline & & & & & & $\begin{array}{rr}0.5 & 0.7 \\
\text { diclofenac }\end{array}$ & $\begin{array}{c}1.52 \\
\text { Favours c }\end{array}$ \\
\hline
\end{tabular}


Comparison 40. Diclofenac gel vs diclofenac+calcitriol

\begin{tabular}{lllll}
\hline Outcome or subgroup title & $\begin{array}{l}\text { No. of } \\
\text { studies }\end{array}$ & $\begin{array}{l}\text { No. of par- } \\
\text { ticipants }\end{array}$ & Statistical method & Effect size \\
\hline 40.1 Early treatment failure & 1 & Risk Ratio (M-H, Random, 95\% Cl) & Totals not selected \\
\hline
\end{tabular}

Analysis 40.1. Comparison 40: Diclofenac gel vs diclofenac+calcitriol, Outcome 1: Early treatment failure

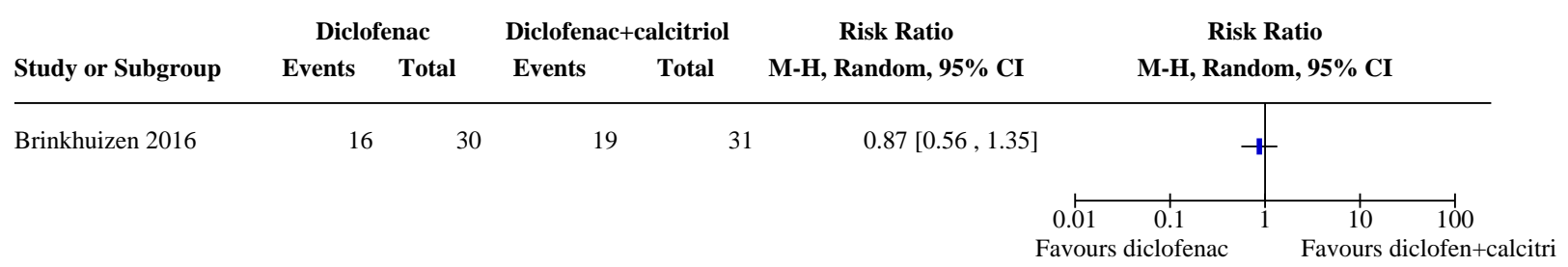

Comparison 41. Calcitriol ointment vs calcitriol+diclofenac

\begin{tabular}{lllll}
\hline Outcome or subgroup title & $\begin{array}{l}\text { No. of } \\
\text { studies }\end{array}$ & $\begin{array}{l}\text { No. of par- } \\
\text { ticipants }\end{array}$ & Statistical method & Effect size \\
\hline 41.1 Early treatment failure & 1 & Risk Ratio (M-H, Random, 95\% Cl) & Totals not selected \\
\hline
\end{tabular}

Analysis 41.1. Comparison 41: Calcitriol ointment vs calcitriol+diclofenac, Outcome 1: Early treatment failure

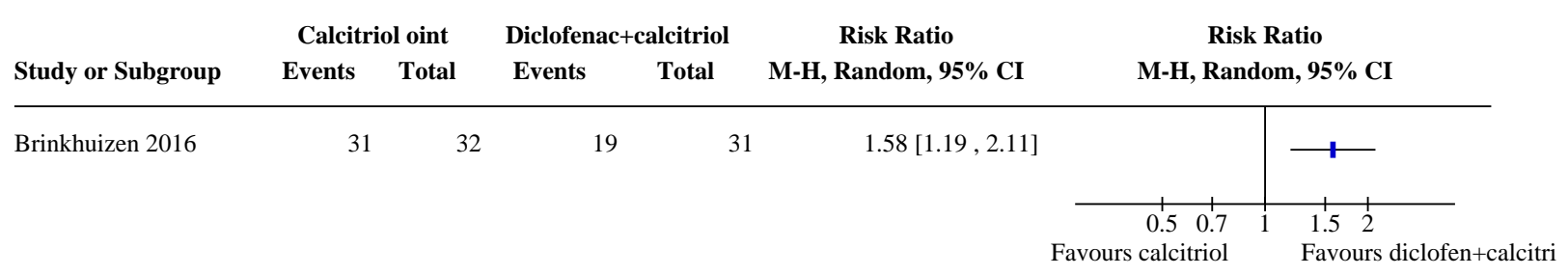

Comparison 42. PDL one session vs PDL two sessions

\begin{tabular}{lllll}
\hline Outcome or subgroup title & $\begin{array}{l}\text { No. of } \\
\text { studies }\end{array}$ & $\begin{array}{l}\text { No. of par- } \\
\text { ticipants }\end{array}$ & Statistical method & Effect size \\
\hline 42.1 Early treatment failure & 1 & Risk Ratio (M-H, Random, 95\% Cl) & Totals not selected \\
\hline
\end{tabular}


Analysis 42.1. Comparison 42: PDL one session vs PDL two sessions, Outcome 1: Early treatment failure

\begin{tabular}{|c|c|c|c|c|c|c|c|}
\hline \multirow[b]{2}{*}{ Study or Subgroup } & \multicolumn{2}{|c|}{ Two sessions } & \multicolumn{2}{|c|}{ One session } & \multirow{2}{*}{$\begin{array}{c}\text { Risk Ratio } \\
\text { M-H, Random, } 95 \% \text { CI }\end{array}$} & \multirow{2}{*}{\multicolumn{2}{|c|}{$\begin{array}{c}\text { Risk Ratio } \\
\text { M-H, Random, 95\% Cl }\end{array}$}} \\
\hline & Events & Total & Events & Total & & & \\
\hline \multirow[t]{2}{*}{ Abd El-Naby 2019} & 9 & 11 & 11 & 11 & $0.83[0.60,1.13]$ & $\longrightarrow$ & - \\
\hline & & & & & $\mathrm{Fa}$ & $\begin{array}{cc} & 1 \\
0.5 & 0.7 \\
\text { vo sessions }\end{array}$ & $\begin{array}{c}1.52 \\
\text { Favours o }\end{array}$ \\
\hline
\end{tabular}

Comparison 43. PDL $7 \mathrm{~mm}$ spot-size single pulses vs PDL $10 \mathrm{~mm}$ spot-size stacked pulses

\begin{tabular}{lllll}
\hline Outcome or subgroup title & $\begin{array}{l}\text { No. of } \\
\text { studies }\end{array}$ & $\begin{array}{l}\text { No. of par- } \\
\text { ticipants }\end{array}$ & Statistical method & Effect size \\
\hline 43.1 Early treatment failure (lesions) & 1 & Risk Ratio (M-H, Random, 95\% Cl) & Totals not selected \\
\hline
\end{tabular}

Analysis 43.1. Comparison 43: PDL $7 \mathrm{~mm}$ spot-size single pulses vs PDL $10 \mathrm{~mm}$ spot-size stacked pulses, Outcome 1: Early treatment failure (lesions)

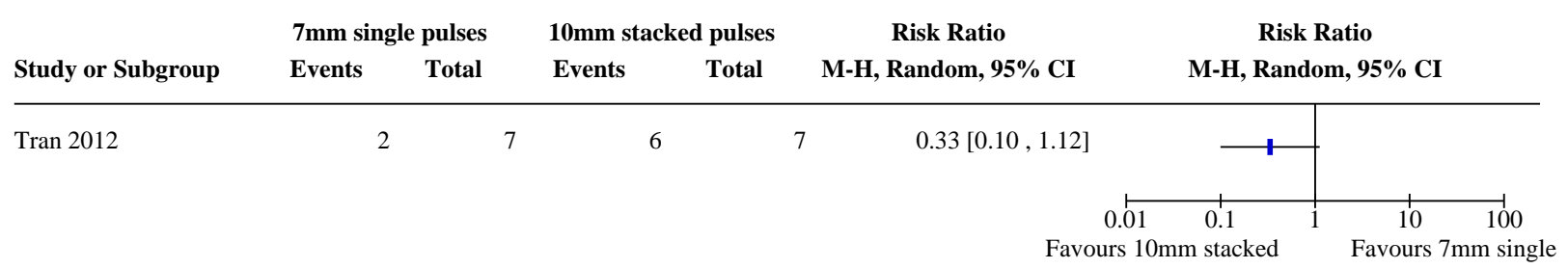

Comparison 44. PDL vs sham laser

\begin{tabular}{lllll}
\hline Outcome or subgroup title & $\begin{array}{l}\text { No. of } \\
\text { studies }\end{array}$ & $\begin{array}{l}\text { No. of par- } \\
\text { ticipants }\end{array}$ & Statistical method & Effect size \\
\hline 44.1 Early treatment failure (lesions) & 1 & Risk Ratio (M-H, Random, 95\% Cl) & Totals not selected \\
\hline
\end{tabular}

Analysis 44.1. Comparison 44: PDL vs sham laser, Outcome 1: Early treatment failure (lesions)

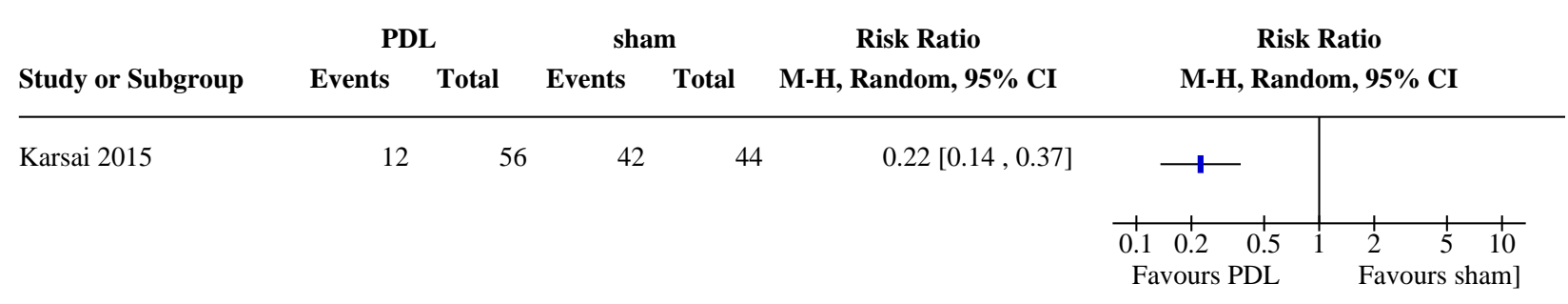


Comparison 45. PDL vs no treatment

\begin{tabular}{llll}
\hline Outcome or subgroup title & $\begin{array}{l}\text { No. of } \\
\text { studies }\end{array}$ & $\begin{array}{l}\text { No. of par- } \\
\text { ticipants }\end{array}$ & Statistical method \\
\hline 45.1 Early treatment failure & 1 & Risk Ratio (M-H, Random, 95\% Cl) & Totals not selected \\
\hline
\end{tabular}

Analysis 45.1. Comparison 45: PDL vs no treatment, Outcome 1: Early treatment failure

\begin{tabular}{|c|c|c|c|c|c|c|c|}
\hline \multirow[b]{2}{*}{ Study or Subgroup } & \multicolumn{2}{|c|}{ PDL } & \multicolumn{2}{|c|}{ No treatment } & \multirow{2}{*}{$\begin{array}{c}\text { Risk Ratio } \\
\text { M-H, Random, 95\% CI }\end{array}$} & \multirow{2}{*}{\multicolumn{2}{|c|}{$\begin{array}{c}\text { Risk Ratio } \\
\text { M-H, Random, 95\% CI }\end{array}$}} \\
\hline & Events & Total & Events & Total & & & \\
\hline Eimpunth 2014 & 4 & 14 & 8 & 10 & $0.36[0.15,0.86]$ & $\longrightarrow$ & \\
\hline & & & & & & $\begin{array}{cc}0.01 & 0.1 \\
\text { Favours PDL }\end{array}$ & $\begin{array}{c}10 \\
1 \\
\text { Favours n }\end{array}$ \\
\hline
\end{tabular}

Comparison 46. Interferon alpha $2 \mathrm{~b}$ vs placebo

\begin{tabular}{lllll}
\hline Outcome or subgroup title & $\begin{array}{l}\text { No. of } \\
\text { studies }\end{array}$ & $\begin{array}{l}\text { No. of par- } \\
\text { ticipants }\end{array}$ & Statistical method & Effect size \\
\hline 46.1 Early treatment failure & 1 & Risk Ratio (M-H, Random, 95\% Cl) & Totals not selected \\
\hline
\end{tabular}

\section{Analysis 46.1. Comparison 46: Interferon alpha 2b vs placebo, Outcome 1: Early treatment failure}

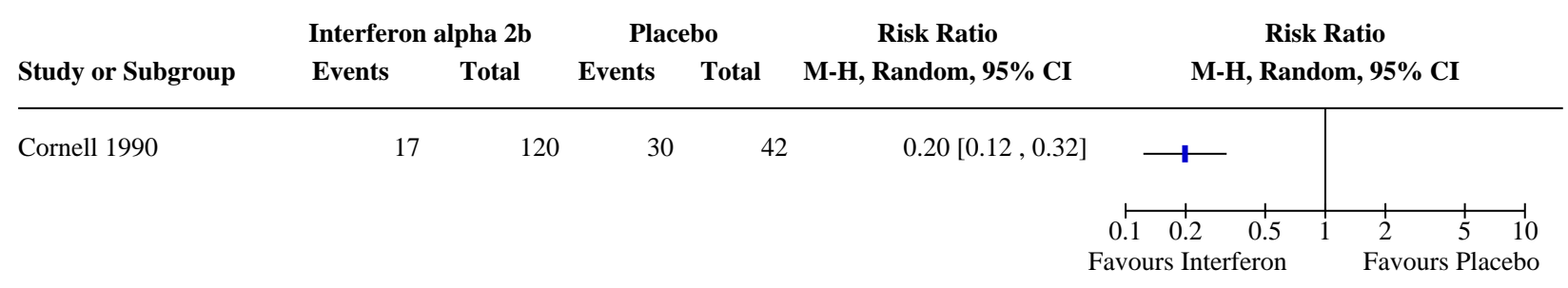

\section{Comparison 47. Interferon beta vs placebo}

\begin{tabular}{lllll}
\hline Outcome or subgroup title & $\begin{array}{l}\text { No. of } \\
\text { studies }\end{array}$ & $\begin{array}{l}\text { No. of par- } \\
\text { ticipants }\end{array}$ & Statistical method & Effect size \\
\hline 47.1 Early treatment failure & 1 & Risk Ratio (M-H, Random, 95\% Cl) & Subtotals only \\
\hline
\end{tabular}


Analysis 47.1. Comparison 47: Interferon beta vs placebo, Outcome 1: Early treatment failure

\begin{tabular}{|c|c|c|c|c|c|c|c|}
\hline \multirow{3}{*}{$\begin{array}{l}\text { Study or Subgroup } \\
\text { Rogozinski } 1997\end{array}$} & \multicolumn{2}{|c|}{ Interferon beta } & \multicolumn{2}{|c|}{ Placebo } & \multirow{2}{*}{$\begin{array}{c}\text { Risk Ratio } \\
\text { M-H, Random, 95\% CI }\end{array}$} & \multirow{2}{*}{\multicolumn{2}{|c|}{$\begin{array}{c}\text { Risk Ratio } \\
\text { M-H, Random, 95\% CI }\end{array}$}} \\
\hline & Events & Total & Events & Total & & & \\
\hline & 8 & 15 & 18 & 18 & $0.55[0.34,0.87]$ & 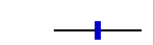 & \\
\hline
\end{tabular}

Comparison 48. Interferon alpha $2 a+2 b$ vs interferon alpha $2 a$

\begin{tabular}{lllll}
\hline Outcome or subgroup title & $\begin{array}{l}\text { No. of } \\
\text { studies }\end{array}$ & $\begin{array}{l}\text { No. of par- } \\
\text { ticipants }\end{array}$ & Statistical method & Effect size \\
\hline 48.1 Early treatment failure & 1 & Risk Ratio (M-H, Random, 95\% Cl) & Subtotals only \\
\hline
\end{tabular}

Analysis 48.1. Comparison 48: Interferon alpha 2a+2b vs interferon alpha 2a, Outcome 1: Early treatment failure

\begin{tabular}{|c|c|c|c|c|c|c|c|}
\hline \multirow[b]{2}{*}{ Study or Subgroup } & \multicolumn{2}{|c|}{ IFN $2 a+2 b$} & \multicolumn{2}{|c|}{ Interferon alpha 2a } & \multirow{2}{*}{$\begin{array}{c}\text { Risk Ratio } \\
\text { M-H, Random, 95\% CI }\end{array}$} & \multirow{2}{*}{\multicolumn{2}{|c|}{$\begin{array}{c}\text { Risk Ratio } \\
\text { M-H, Random, 95\% CI }\end{array}$}} \\
\hline & Events & Total & Events & Total & & & \\
\hline Alpsoy 1996 & 4 & 15 & 5 & 15 & $0.80[0.27,2.41]$ & $\longrightarrow$ & - \\
\hline & & & & & & $\begin{array}{lr} & 1 \\
.2 & 0.5 \\
\text { FN } 2 a+2 b\end{array}$ & $\begin{array}{ccc}1 & 1 & 10 \\
2 & 5 & 10 \\
\text { Favours } & \text { IFN } & 2 \mathrm{a}\end{array}$ \\
\hline
\end{tabular}

Comparison 49. Interferon alpha $2 a+2 b$ vs interferon alpha $2 b$

\begin{tabular}{lllll}
\hline Outcome or subgroup title & $\begin{array}{l}\text { No. of } \\
\text { studies }\end{array}$ & $\begin{array}{l}\text { No. of par- } \\
\text { ticipants }\end{array}$ & Statistical method & Effect size \\
\hline 49.1 Early treatment failure & 1 & Risk Ratio (M-H, Random, 95\% Cl) & Subtotals only \\
\hline
\end{tabular}

Analysis 49.1. Comparison 49: Interferon alpha $2 a+2 b$ vs interferon alpha $2 b$, Outcome 1: Early treatment failure

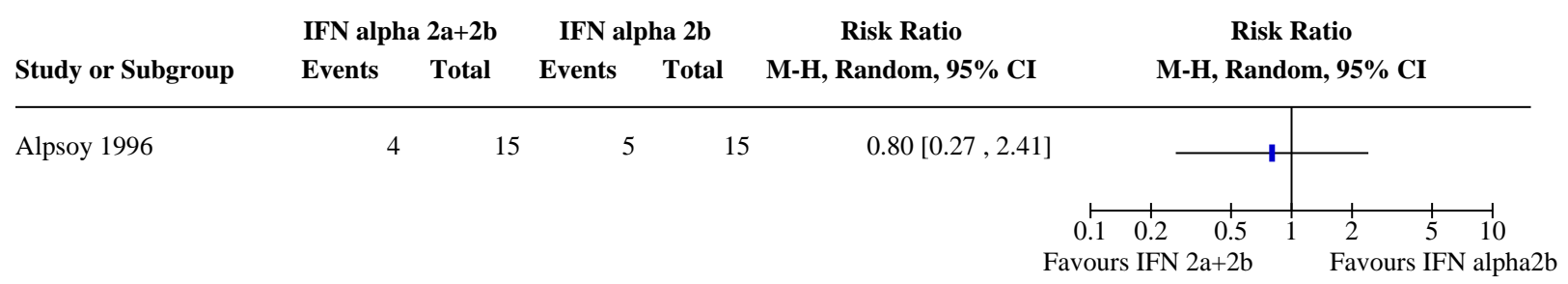


Comparison 50 . Interferon alpha $2 \mathrm{~b}$ vs interferon alpha $2 \mathrm{a}$

\begin{tabular}{llll}
\hline Outcome or subgroup title & $\begin{array}{l}\text { No. of } \\
\text { studies }\end{array}$ & $\begin{array}{l}\text { No. of par- } \\
\text { ticipants }\end{array}$ & Statistical method \\
\hline 50.1 Early treatment failure & 1 & Risk Ratio (M-H, Random, $95 \% \mathrm{Cl})$ \\
\hline
\end{tabular}

Analysis 50.1. Comparison 50: Interferon alpha $2 \mathrm{~b}$ vs interferon alpha 2a, Outcome 1: Early treatment failure

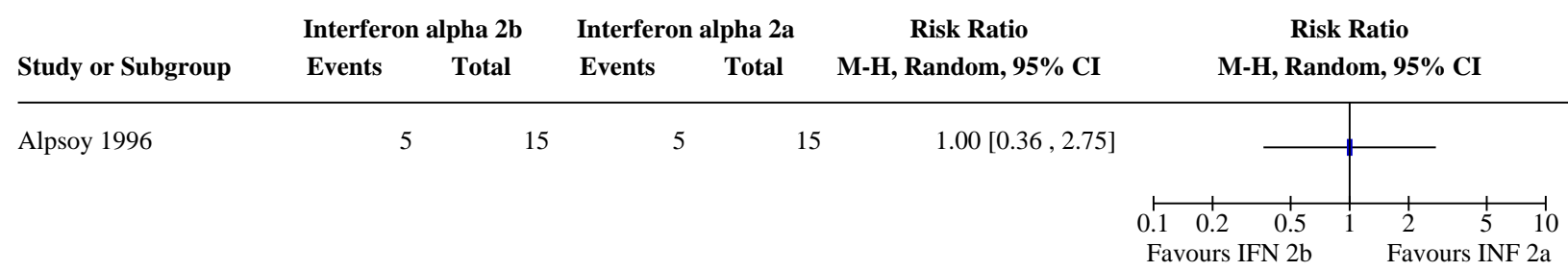

Comparison 51 . $3 x$ a week vs single dose protamine zinc chelate IFN alpha $2 b$

\begin{tabular}{lllll}
\hline Outcome or subgroup title & $\begin{array}{l}\text { No. of } \\
\text { studies }\end{array}$ & $\begin{array}{l}\text { No. of par- } \\
\text { ticipants }\end{array}$ & Statistical method & Effect size \\
\hline 51.1 Early treatment failure & 1 & & Risk Ratio (M-H, Random, 95\% Cl) & Subtotals only \\
\hline
\end{tabular}

Analysis 51.1. Comparison 51: $3 x$ a week vs single dose protamine zinc chelate IFN alpha 2 b, Outcome 1: Early treatment failure

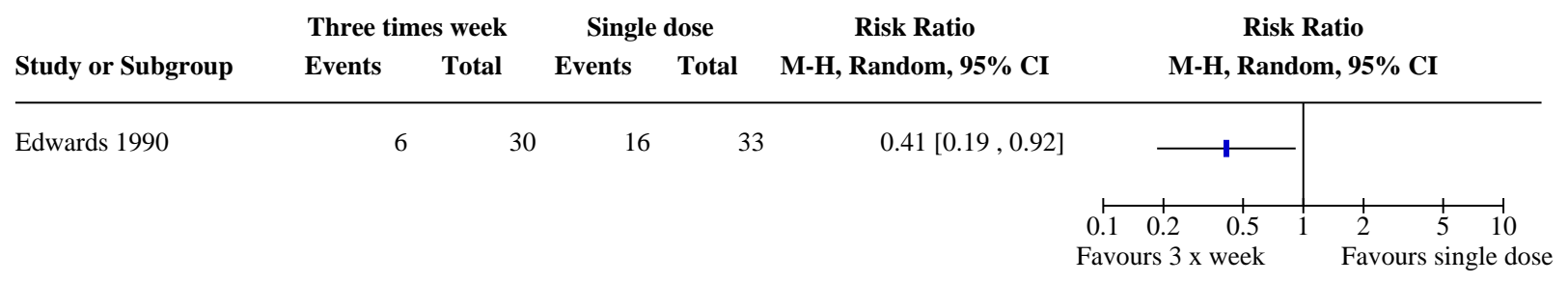

\section{Comparison 52. Topical sinecatechin vs placebo}

\begin{tabular}{lllll}
\hline Outcome or subgroup title & $\begin{array}{l}\text { No. of } \\
\text { studies }\end{array}$ & $\begin{array}{l}\text { No. of par- } \\
\text { ticipants }\end{array}$ & Statistical method & Effect size \\
\hline 52.1 Early treatment failure & 1 & Risk Ratio $(\mathrm{M}-\mathrm{H}$, Random, $95 \% \mathrm{Cl})$ & Totals not selected \\
\hline
\end{tabular}


Analysis 52.1. Comparison 52: Topical sinecatechin vs placebo, Outcome 1: Early treatment failure

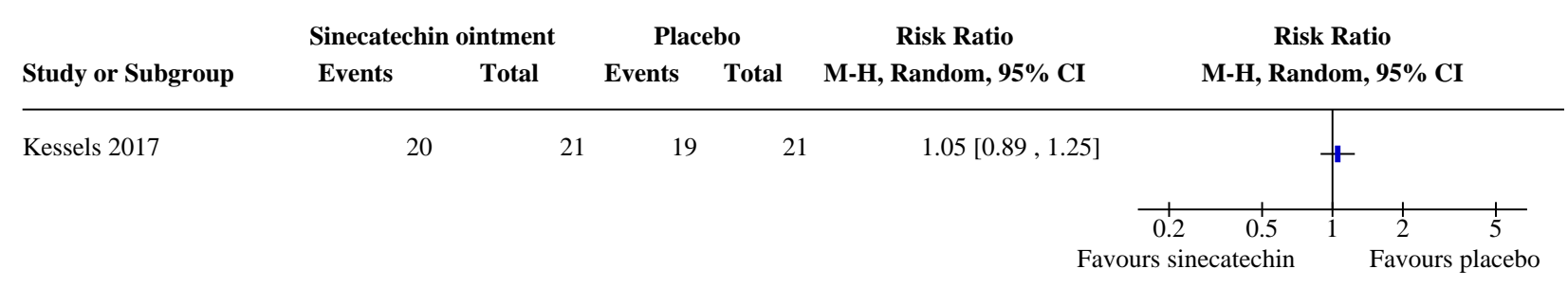

ADDITIONAL TABLES

Table 1. Pain table

\begin{tabular}{|c|c|c|c|c|}
\hline $\begin{array}{l}\text { Study (com- } \\
\text { parator) }\end{array}$ & Analgesia & Assessment & Severe pain & Other notable outcomes \\
\hline $\begin{array}{l}\text { Abd El-Na- } \\
\text { by } 2019 \text { (one } \\
\text { PDL session / } \\
\text { two PDL ses- } \\
\text { sions) }\end{array}$ & Not stated. & Not stated. & Not stated. & $\begin{array}{l}\text { Only } 2 \text { patients experienced pain and ulceration in the } \\
\text { single-session group. There was no significant differ- } \\
\text { ence between the two groups. }\end{array}$ \\
\hline $\begin{array}{l}\text { Alpsoy } 1996 \\
\text { (Interferon } \\
\text { alpha 2a / } \\
2 b / 2 a \& b)\end{array}$ & Not stated. & Not stated. & Not stated. & $\begin{array}{l}\text { Transient local discomfort at injection site (requiring } \\
\text { no treatment) experienced in all patients during injec- } \\
\text { tion. }\end{array}$ \\
\hline $\begin{array}{l}\text { Arits } 2013 \\
\text { (MAL PDT / } \\
\text { Imiquimod / } \\
\text { 5-FU) }\end{array}$ & Not stated. & $\begin{array}{l}\text { Weekly post-treatment } \\
\text { patient-reported maxi- } \\
\text { mum score for pain and } \\
\text { burning sensation on a } \\
\text { visual analogue scale (0- } \\
\text { 10) for six weeks, and } \\
\text { maximum score during } \\
\text { the first and second pho- } \\
\text { todynamic therapy ses- } \\
\text { sions. Categorised as 0-3 } \\
=\text { mild, } 4-6 \text { moderate, } \\
7-10=\text { severe. }\end{array}$ & $\begin{array}{l}\text { Highest over- } \\
\text { all percentage } \\
\text { experienced } \\
\text { pain during } \\
\text { second PDT } \\
\text { treatment } \\
(10 \%) \text {. High- } \\
\text { est severe } \\
\text { pain rating in } \\
\text { imiquimod } \\
\text { group found } \\
\text { at week } 6 \text { ( } 7 \%) \\
\text { and in Fluo- } \\
\text { rouracil group } \\
\text { at week } 4 \\
\text { (4\%). }\end{array}$ & $\begin{array}{l}\text { Maximum duration of pain and burning sensation: } \\
\text { Imiquimod group }=6 \text { weeks, Fluorouracil group }=4 \\
\text { weeks, PDT group }=2 \text { weeks. }\end{array}$ \\
\hline $\begin{array}{l}\text { Bas- } \\
\text { set-Seguin } \\
2008 \text { (MAL- } \\
\text { PDT / } \\
\text { cryosurgery) }\end{array}$ & Not stated. & $\begin{array}{l}\text { AEs at each follow-up } \\
\text { visit up to three months } \\
\text { post-treatment accord- } \\
\text { ing to three-point sever- } \\
\text { ity scale (mild/moder- } \\
\text { ate/severe). }\end{array}$ & $\begin{array}{l}1 \% \text { in } \\
\text { cryosurgery } \\
\text { group and } 5 \% \\
\text { in MAL- PDT } \\
\text { group. }\end{array}$ & $\begin{array}{l}\text { Local pain in } 33 \% \text { in cryosurgery group compared to } \\
37 \% \text { in MAL-PDT group. }\end{array}$ \\
\hline $\begin{array}{l}\text { Bath-Hex- } \\
\text { tall } 2014 \\
\text { (Imiquimod / } \\
\text { SE) }\end{array}$ & $\begin{array}{l}\text { Routine } \\
\text { analgesia af- } \\
\text { ter surgery. } 1 \\
\text { week break } \\
\text { and restart } \\
\text { at lower fre- }\end{array}$ & $\begin{array}{l}\text { During treatment and at } \\
16 \text { weeks using six-point } \\
\text { scale. Number of days of } \\
\text { moderate to severe pain. }\end{array}$ & $\begin{array}{l}30 \% \text { of pa- } \\
\text { tients in the } \\
\text { imiquimod } \\
\text { group and } \\
22 \% \text { patients } \\
\text { in the SE }\end{array}$ & $\begin{array}{l}\text { More patients reported pain during treatment in im- } \\
\text { iquimod group }\end{array}$ \\
\hline
\end{tabular}


Table 1. Pain table (Continued)

quency of
application
if unable
to tolerate
Imiquimod.

group had moderate or severe pain at some time during treatment.

$9 \%$ of patients treated with imiquimod had moderate or severe pain in the 16 weeks after treatment compared with $20 \%$ of patients who underwent SE.

Brinkhuizen Not stated.
2016 (di-
clofenac /

\section{Weekly patient-reported \\ Not stated.} pain score on a VAS $(0-$ 10).
Pain at application site most likely to occur in the combination therapy group (40.6\%) as compared to the diclofenac group (25.8\%) and the calcitriol group (12.5\%).

\begin{tabular}{|c|c|c|c|c|}
\hline $\begin{array}{l}\text { Choi } 2016 \\
\text { (Er:YAG AFL- } \\
\text { PDT / MAL- } \\
\text { PDT) }\end{array}$ & $\begin{array}{l}\text { Anaesthet- } \\
\text { ic cream pri- } \\
\text { or to proce- } \\
\text { dure. }\end{array}$ & $\begin{array}{l}\text { VAS scores (11-point } \\
\text { scale) during illumina- } \\
\text { tion. }\end{array}$ & Not stated. & $\begin{array}{l}\text { All patients experienced mild to moderate pain during } \\
\text { PDT illumination. After illumination had ceased, the } \\
\text { pain intensity immediately lessened, resolving over } \\
\text { the next few hours. VAS scores (11-point scale) during } \\
\text { illumination were similar with Er:YAG AFL-PDT ( } 4.632 \\
+/-1.257) \text { and MAL-PDT }(4.222+/-1.865 ; P=0.437) \text {. }\end{array}$ \\
\hline
\end{tabular}

\begin{tabular}{|c|c|c|c|c|}
\hline $\begin{array}{l}\text { Ezughah } \\
2008 \\
\text { (Imiquimod } \\
8 \text { weeks / } 5 \\
\text { weeks) }\end{array}$ & $\begin{array}{l}\text { Patients } \\
\text { with intol- } \\
\text { erable reac- } \\
\text { tions were } \\
\text { allowed a } \\
\text { rest period. }\end{array}$ & $\begin{array}{l}\text { Weekly visual analogue } \\
\text { scale scores (10-point } \\
\text { scale). }\end{array}$ & $\begin{array}{l}\text { One patient } \\
\text { in 8-week arm } \\
\text { failed to com- } \\
\text { plete course } \\
\text { due to severe } \\
\text { pain. }\end{array}$ & $\begin{array}{l}\text { Composite weekly median VAS scores for tolerabili- } \\
\text { ty are low, suggesting little discomfort in both study } \\
\text { arms; there was no overall difference between the } \\
\text { two groups. }\end{array}$ \\
\hline $\begin{array}{l}\text { Foley } \\
\text { 2009a/Foley } \\
\text { 2009b (MAL- } \\
\text { PDT / place- } \\
\text { bo) }\end{array}$ & Not stated. & $\begin{array}{l}\text { Adverse effects noted } \\
\text { at each follow-up visit } \\
\text { and rated as mild/mod- } \\
\text { erate/severe. }\end{array}$ & Not stated. & $\begin{array}{l}\text { Burning more common than stinging or other skin } \\
\text { pain. } 29 \% \text { in treatment arm compared to } 12 \% \text { in } \\
\text { placebo group. Pain }=18 \% \text { and } 5 \% \text {, respectively, } \\
\text { stinging }=15 \% \text { and } 8 \% \text {, respectively. }\end{array}$ \\
\hline $\begin{array}{l}\text { Garcia-Mar- } \\
\text { tin } 2011 \\
\text { (Imiquimod / } \\
\text { radiothera- } \\
\text { py) }\end{array}$ & $\begin{array}{l}\text { Tobramycin } \\
\text { and dexam- } \\
\text { ethasone } \\
\text { drops sup- } \\
\text { plied if pain } \\
\text { reported. }\end{array}$ & $\begin{array}{l}\text { Adverse effects noted at } \\
\text { each follow-up visit. }\end{array}$ & $\begin{array}{l}\text { Intense con- } \\
\text { junctival ir- } \\
\text { ritation re- } \\
\text { ported in } 2 \\
\text { patents in the } \\
\text { Imiquimod } \\
\text { group. }\end{array}$ & $\begin{array}{l}50 \% \text { of the patients in the radiotherapy group com- } \\
\text { pared to } 60 \% \text { in the imiquimod group reported symp- } \\
\text { toms of discomfort with blinking during treatment. } \\
\text { One patient complained of slight pain in the lower } \\
\text { eyelid during radiotherapy treatment. }\end{array}$ \\
\hline $\begin{array}{l}\text { Geisse } 2002 \\
\text { (Imiquimod } \\
\mathrm{BD} / \mathrm{OD} /\end{array}$ & $\begin{array}{l}\text { Rest peri- } \\
\text { od of up to }\end{array}$ & $\begin{array}{l}\text { Patient-reported adverse } \\
\text { effects at each visit. }\end{array}$ & Not stated. & $\begin{array}{l}\text { Pain and tenderness at the target site were reported } \\
\text { less frequently than itching. }\end{array}$ \\
\hline
\end{tabular}


Table 1. Pain table (Continued)

5xpw / 14 days al-

3xpw/vehi- lowed.

cle)

$\begin{array}{lllll}\begin{array}{l}\text { Geisse 2004 } \\ \text { (Imiquimod }\end{array} & \begin{array}{l}\text { Rest period } \\ \text { allowed dur- }\end{array} & \begin{array}{l}\text { Patient-reported adverse } \\ \text { effects at each visit. }\end{array} & \begin{array}{l}\text { Patient-re- } \\ \text { ported ad- } \\ \text { ing which }\end{array} & \text { Not stated. } \\ \begin{array}{ll}\text { ixpw / vehi- } \\ \text { cle) }\end{array} & \begin{array}{l}\text { light dress- } \\ \text { ing / emol- }\end{array} & & \\ & \begin{array}{l}\text { at each visit. } \\ \text { lient could } \\ \text { be used. }\end{array} & & \\ & & & \end{array}$

\begin{tabular}{|c|c|c|c|c|}
\hline $\begin{array}{l}\text { Haak } 2015 \\
\text { (AFXL-PDT / } \\
\text { MAL-PDT) }\end{array}$ & $\begin{array}{l}\text { Mepivacaine } \\
10 \mathrm{mg} / \mathrm{mL} \text { in- } \\
\text { jected pri- } \\
\text { or to proce- } \\
\text { dure. }\end{array}$ & $\begin{array}{l}\text { Pain scores during LED il- } \\
\text { lumination, scored from } \\
0-10 .\end{array}$ & Not stated. & $\begin{array}{l}\text { "AFXL pre-treatment did not influence median pain } \\
\text { scores during LED illumination and pain intensities } \\
\text { were similar at first and second illumination: first } \\
\text { treatment AFXL-PDT median } 3 \text { (IQR 2-55) vs MAL-PDT } \\
35 \text { ( } 25-5) \text {, second treatment AFXL-PDT } 35 \text { (3-65) vs } \\
\text { MAL-PDT } 3 \text { (3-45), (P>0519). The presumed enhanced } \\
\text { uptake of MAL did not affect the pain level during illu- } \\
\text { mination. Pain scores were similar during AFXL-PDT } \\
\text { and MAL-PDT and rated as mild to moderate." }\end{array}$ \\
\hline
\end{tabular}

\begin{tabular}{|c|c|c|c|c|}
\hline $\begin{array}{l}\text { Karsai } 2015 \\
\text { (PDL / sham) }\end{array}$ & Not stated. & $\begin{array}{l}\text { Pain intensity using a VAS } \\
(0 \mathrm{~cm}=\text { 'no pain' to } 10 \\
\mathrm{cm}=\text { 'maximal pain') at } \\
\text { every visit. }\end{array}$ & Not stated. & $\begin{array}{l}\text { "The following median values were indicated on the } \\
\text { pain intensity scale (ordinal scale } 0-10) \text { among pa- } \\
\text { tients at the four treatment sessions surveyed (mini- } \\
\text { mum-maximum): Laser treatment: } 2(0-6), 3(0-6), 3 \\
(0-8) \text { and } 4(0-9) \text {; Sham treatment: } 0(0-1), 0(0-1), 0 \\
(0-1) \text { and } 0(0-2) \text {." }\end{array}$ \\
\hline
\end{tabular}

\begin{tabular}{|c|c|c|c|c|}
\hline $\begin{array}{l}\text { Kessels } 2017 \\
\text { (Sinecate- } \\
\text { chins / place- } \\
\text { bo) }\end{array}$ & $\begin{array}{l}\text { Oral parac- } \\
\text { etamol for } \\
\text { local skin re- } \\
\text { actions (if se- }\end{array}$ & $\begin{array}{l}\text { Subjective symptoms in- } \\
\text { cluding pain were record- } \\
\text { ed in personal diary kept } \\
\text { by patients once a week }\end{array}$ & Not stated. & $\begin{array}{l}\text { A burning sensation was reported in the treatment } \\
\text { group in } 5 \% \text { of patients, at week four compared to } 1 \% \\
\text { in the treatment group. }\end{array}$ \\
\hline
\end{tabular}
vere treat-

be stopped for 1 week)

\begin{tabular}{|c|c|c|c|c|}
\hline $\begin{array}{l}\text { Kessels } 2018 \\
\text { (MAL-PDT / } \\
\text { two-fold } \\
\text { fractionated } \\
\text { ALA-PDT) }\end{array}$ & Not stated. & $\begin{array}{l}\text { Pain and burning sensa- } \\
\text { tion } \\
\text { were scored using a } \\
\text { numerical rating scale } \\
\text { (score } 0-10) \text {, } \\
\text { directly after both illumi- } \\
\text { nations and } 1 \text { week later. } \\
\text { The maximum } \\
\text { pain scores for both illu- } \\
\text { minations were assessed. }\end{array}$ & Not stated. & $\begin{array}{l}\text { "After the second illumination, mean pain scores were } \\
\text { significantly higher in the two-fold ALA-PDT group } \\
\text { compared with patients treated with MAL-PDT, with } \\
\text { mean pain scores of } 3.36+-2.57 \text { and } 2.48+-2.57 \text { re- } \\
\text { spectively }(\mathrm{P}=0.039) \text {. None of the patients discontin- } \\
\text { ued treatment because of pain. } 16.4 \% \text { in the ALA-PDT } \\
\text { group vs } 5.8 \% \text { in the MAL-PDT group reported the use } \\
\text { of pain medication post-treatment." }\end{array}$ \\
\hline $\begin{array}{l}\text { Kuijpers } \\
2006 \text { (ALA- } \\
\text { PDT / MAL- } \\
\text { PDT) }\end{array}$ & Not stated. & $\begin{array}{l}\text { Patient description of } \\
\text { pain and rating on } 10- \\
\text { point visual analogue } \\
\text { scale. }\end{array}$ & $\begin{array}{l}\text { None report- } \\
\text { ed. }\end{array}$ & $\begin{array}{l}\text { Majority of pain was reported as burning or stinging } \\
\text { ( } 59 \text { and } 41 \% \text { respectively); also described as throb- } \\
\text { bing, lingering or tingling. Intensity and character was } \\
\text { the same between groups. Generally pain was experi- } \\
\text { enced during illumination and sporadically after, with } \\
\text { the second cycle reported as the worst. No radiation } \\
\text { reported. }\end{array}$ \\
\hline
\end{tabular}


Table 1. Pain table (Continued)

\begin{tabular}{|c|c|c|c|c|}
\hline $\begin{array}{l}\text { Marks } 2001 \\
\text { (Imiquimod } \\
\text { BD / OD / BD } \\
\text { for } 3 \text { days / } \\
\text { OD for } 2 \\
\text { days) }\end{array}$ & Not stated. & $\begin{array}{l}\text { Three-point rating scale } \\
\text { applied by assessor } \\
\text { based on descriptive } \\
\text { terms used by patient. }\end{array}$ & Not stated. & $\begin{array}{l}\text { Pain more frequent in the BD group }(100 \%)>\text { OD } \\
\text { group }(24.2 \%)>\text { BD for three days }(13.3 \%)>\text { OD for } 2 \\
\text { days }(3 \%) \text {. }\end{array}$ \\
\hline
\end{tabular}

\begin{tabular}{|c|c|c|c|c|}
\hline $\begin{array}{l}\text { Miller } 1997 \\
\text { (Intrale- } \\
\text { sional sus- } \\
\text { tained-re- } \\
\text { lease 6-fluo- } \\
\text { rouracil / ep- } \\
\text { inephrine in- } \\
\text { jectable gel, } \\
\text { variable fre- } \\
\text { quencies) }\end{array}$ & $\begin{array}{l}\text { Reduced } \\
\text { dose if } \\
\text { tenderness } \\
\text { or pain at } \\
\text { the injection } \\
\text { site or ero- } \\
\text { sion/ } \\
\text { ulceration. } \\
\text { Local anaes- } \\
\text { thetic not } \\
\text { permitted. }\end{array}$ & $\begin{array}{l}\text { At each treatment visit, } \\
\text { patients were asked to } \\
\text { report injection site } \\
\text { pain, including burning } \\
\text { or stinging, and tender- } \\
\text { ness. }\end{array}$ & $\begin{array}{l}\text { All patients } \\
\text { experienced } \\
\text { transient } \\
\text { moderate to } \\
\text { severe sting- } \\
\text { ing, burning } \\
\text { or pain at the } \\
\text { time of injec- } \\
\text { tion lasting } \\
\text { less than } 15 \\
\text { to } 20 \text { minutes } \\
\text { after drug ad- } \\
\text { ministration. }\end{array}$ & Not stated. \\
\hline
\end{tabular}

\begin{tabular}{|c|c|c|c|c|}
\hline $\begin{array}{l}\text { Morton } 2018 \\
\text { (BF } 200 \text { ALA- } \\
\text { PDT / MAL- } \\
\text { PDT) }\end{array}$ & $\begin{array}{l}\text { Pain man- } \\
\text { agement: } \\
\text { physical } \\
\text { cooling mea- } \\
\text { sures, reduc- } \\
\text { tion of light } \\
\text { intensity } \\
\text { at the ex- } \\
\text { pense of } \\
\text { longer expo- } \\
\text { sure times, } \\
\text { or "slight } \\
\text { analgesia". }\end{array}$ & $\begin{array}{l}\text { Local pain experienced } \\
\text { during PDT was assessed } \\
\text { for each PDT session (on } \\
\text { a numerical rating pain } \\
\text { scale). } \\
\text { Ranking of the subjective } \\
\text { sensations } \\
\text { pain, burning and itching } \\
\text { was done by the patient. } \\
\text { Pain during } \\
\text { PDT assessed with a nu- } \\
\text { merical rating pain scale } \\
\text { ranging } \\
\text { from } 0 \text { (no pain at all) to } \\
10 \text { (worst possible pain). }\end{array}$ & Not stated. & $\begin{array}{l}\text { Pain was one of the most frequently reported reac- } \\
\text { tions, experienced by } 100 \% \text { patients undergoing MAL- } \\
\text { PDT and } 97.1 \% \text { patients undergoing BF } 200 \text { ALA-PDT. } \\
\text { Local pain experienced during each session showed } \\
\text { little variation between both treatments and was } \\
\text { most commonly experienced during the first two ses- } \\
\text { sions. }\end{array}$ \\
\hline $\begin{array}{l}\text { Rhodes } 2004 \\
\text { (MAL-PDT / } \\
\text { SE) }\end{array}$ & $\begin{array}{l}\text { None in MAL- } \\
\text { PDT group, } \\
\text { local anaes- } \\
\text { thetic in SE } \\
\text { group. }\end{array}$ & $\begin{array}{l}\text { Local skin reactions dur- } \\
\text { ing and after treatment } \\
\text { at each clinic visit were } \\
\text { documented, and rated } \\
\text { as mild, moderate or se- } \\
\text { vere. }\end{array}$ & $\begin{array}{l}\text { One patient } \\
\text { in MAL-PDT } \\
\text { group discon- } \\
\text { tinued treat- } \\
\text { ment because } \\
\text { of severe } \\
\text { burning sen- } \\
\text { sation which } \\
\text { resolved with- } \\
\text { out medical } \\
\text { intervention. }\end{array}$ & $\begin{array}{l}14 \% \text { in the MAL-PDT group experienced pain, com- } \\
\text { pared to } 6 \% \text { in the SE group. }\end{array}$ \\
\hline
\end{tabular}


Table 1. Pain table (Continued)

reaction oc-

curred.

\begin{tabular}{|c|c|c|c|c|}
\hline $\begin{array}{l}\text { Shumack } \\
2002 \mathrm{~b} \\
\text { (Imiquimod, } \\
\text { several } \\
\text { regimes for } \\
12 \text { weeks) }\end{array}$ & $\begin{array}{l}\text { Maximum of } \\
\text { two 7-day } \\
\text { periods of } \\
\text { rest allowed } \\
\text { if local skin } \\
\text { reaction oc- } \\
\text { curred. }\end{array}$ & $\begin{array}{l}\text { Adverse effects docu- } \\
\text { mented at every study } \\
\text { visit. }\end{array}$ & $\begin{array}{l}\text { Two patients } \\
\text { from the im- } \\
\text { iquimod twice } \\
\text { daily } 7 \text {-day } \\
\text { dosing group } \\
\text { reported se- } \\
\text { vere applica- } \\
\text { tion site re- } \\
\text { actions, one } \\
\text { with pain at } \\
\text { the target } \\
\text { site and one } \\
\text { with tender- } \\
\text { ness, sting- } \\
\text { ing and pain } \\
\text { at the target } \\
\text { site. One pa- } \\
\text { tient from the } \\
\text { once-daily, } 7 \\
\text { day group re- } \\
\text { ported severe } \\
\text { tenderness at } \\
\text { the target site. } \\
\text { One patient } \\
\text { discontinued } \\
\text { due to pain, } \\
\text { tenderness } \\
\text { and drainage } \\
\text { at the target } \\
\text { site. }\end{array}$ & Not stated. \\
\hline
\end{tabular}

Siller 2010
(ingenol
mebutate
gel 0.0025
$\% /$ ingenol
mebutate
gel $0.01 \%$ /
ingenol
mebutate gel
$0.05 \%$ / vehi-
cle)

Treatment Adverse effects docu- Not stated.
was withheld mented at every study if there was visit. a severe local reaction occurred after the first dose.

\begin{tabular}{|c|c|c|c|c|}
\hline $\begin{array}{l}\text { Soler } 2000 \\
\text { (Laser light / } \\
\text { broadband } \\
\text { light) }\end{array}$ & Not stated. & $\begin{array}{l}\text { Pain reported during } \\
\text { treatment and in fol- } \\
\text { low-up period (patient } \\
\text { questionnaires). }\end{array}$ & Not stated. & $\begin{array}{l}83 \% \text { of patients in the laser group and } 76 \% \text { in the } \\
\text { broadband group reported some discomfort during } \\
\text { and after illumination. Types of pain included stinging } \\
\text { and burning sensation. There was no significant dif- } \\
\text { ference between the groups. }\end{array}$ \\
\hline $\begin{array}{l}\text { Sterry } \\
\text { 2002a/Ster- } \\
\text { ry 2002b } \\
\text { (Imiquimod } \\
\text { with / with- } \\
\text { out occlu- } \\
\text { sion) }\end{array}$ & $\begin{array}{l}\text { Prescribed } \\
\text { rest periods } \\
\text { from treat- } \\
\text { ment } \\
\text { if local skin } \\
\text { reactions or } \\
\text { treatment }\end{array}$ & $\begin{array}{l}\text { Adverse effects docu- } \\
\text { mented at every study } \\
\text { visit. }\end{array}$ & Not stated. & $\begin{array}{l}\text { One patient reported continuous stinging and pain } \\
\text { at the application site; one reported irritation and an- } \\
\text { other reported irritation and pruritus. }\end{array}$ \\
\hline
\end{tabular}


Table 1. Pain table (Continued)

site adverse

effects.

\begin{tabular}{|c|c|c|c|c|}
\hline $\begin{array}{l}\text { Szeimies } \\
2008 \text { (MAL- } \\
\text { PDT / SE) }\end{array}$ & $\begin{array}{l}\text { Cooling of } \\
\text { irradiation } \\
\text { sites with } \\
\text { fan-cooler } \\
\text { during treat- } \\
\text { ment. }\end{array}$ & $\begin{array}{l}\text { Adverse effects docu- } \\
\text { mented at every study } \\
\text { visit. }\end{array}$ & Not stated. & $\begin{array}{l}\text { Post procedural pain was reported in } 3 \text { of the surgical } \\
\text { participants and none of the MAL-PDT group. Pain was } \\
\text { reported in } 2 \text { and } 1 \text { of the participants in the MAL-PDT } \\
\text { and surgical groups respectively. }\end{array}$ \\
\hline $\begin{array}{l}\text { Wang } 2001 \\
\text { (ALA-PDT / } \\
\text { cryosurgery) }\end{array}$ & $\begin{array}{l}\text { In the } \\
\text { PDT group, } \\
\text { low fluence } \\
\text { rate to min- } \\
\text { imise ther- } \\
\text { mal effects } \\
\text { and lesions } \\
\text { sprayed with } \\
\text { water. }\end{array}$ & $\begin{array}{l}\text { Pain recorded in first } \\
\text { week after treatment us- } \\
\text { ing } 115-\mathrm{mm} \text { visual ana- } \\
\text { logue scale (VAS) for pain } \\
\text { indication and analgesia } \\
\text { diary. }\end{array}$ & Not stated. & $\begin{array}{l}\text { Overall VAS scores low for both treatment modalities, } \\
\text { and insignificantly higher in PDT group }(43+/-31 \mathrm{~mm} \\
\text { SD in PDT group, } 32+/-27 \mathrm{~mm} \text { SD in cryosurgery } \\
\text { group). Most pain reported during treatment. }\end{array}$ \\
\hline $\begin{array}{l}\text { 2005-001474-27 } \\
\text { (placebo gel } \\
+ \text { tazarotene } \\
\text { gel / valproic } \\
\text { acid gel + } \\
\text { tazarotene } \\
\text { gel) }\end{array}$ & 7 Not stated. & Not stated. & Not stated. & $\begin{array}{l}8 \text { patients in the placebo group experienced pain in } \\
\text { comparison to } 6 \text { in the treatment group. }\end{array}$ \\
\hline
\end{tabular}

MAL: methyl aminolevulinate; PDL: pulsed dye laser; PDT: photodynamic therapy; SE: surgical excision; VAS: visual analogue scale; xpw: times per week.

Table 2. Adverse effects table

\begin{tabular}{|c|c|c|c|c|}
\hline $\begin{array}{l}\text { Study } \\
\text { (com- } \\
\text { parator) }\end{array}$ & $\begin{array}{l}\text { AEs associat- } \\
\text { ed with treat- } \\
\text { ment leading } \\
\text { to discontinu- } \\
\text { ation of treat- } \\
\text { ment / partici- } \\
\text { pation }\end{array}$ & $\begin{array}{l}\text { Common AEs (number/on- } \\
\text { set/duration) }\end{array}$ & $\begin{array}{l}\text { Other notable outcomes (num- } \\
\text { ber/onset/duration) }\end{array}$ & Comments \\
\hline $\begin{array}{l}\text { Abd El-Na- } \\
\text { by } 2019 \\
\text { (one PDL } \\
\text { session / } \\
\text { two PDL } \\
\text { sessions) }\end{array}$ & Not stated. & $\begin{array}{l}\text { In the group who received } \\
\text { two sessions of treatment, } 4 \\
\text { patients experienced dyspig- } \\
\text { mentation ( } 2 \text { hyper- and } 2 \text { hy- } \\
\text { po-pigmentation). }\end{array}$ & Not stated. & \\
\hline $\begin{array}{l}\text { Arits } 2013 \\
\text { (MAL- } \\
\text { PDT / } \\
\text { Imiquimod / } \\
\text { 5-FU) }\end{array}$ & None reported. & $\begin{array}{l}\text { Local skin redness reported } \\
\text { as moderate or severe in all } \\
\text { treatment groups. } \\
\text { Patients treated with im- } \\
\text { iquimod or 5-FU more often } \\
\text { reported moderate to severe } \\
\text { local swelling, erosion, crust } \\
\text { formation and itching of the }\end{array}$ & $\begin{array}{l}\text { Unexpected serious adverse ef- } \\
\text { fects in } 1) \text { Imiquimod group in- } \\
\text { clude wound infection }(n=1) \text { and } \\
\text { influenza-like symptoms }(n=8) \\
\text { and } 2) \text { in } 5 \text {-FU group include two } \\
\text { local wound infections, erysipelas } \\
\text { of the lower extremity }(n=1) \text {, leg } \\
\text { ulcer }(n=1) \text {. }\end{array}$ & $\begin{array}{l}\text { Adverse reactions more com- } \\
\text { mon in last treatment weeks. }\end{array}$ \\
\hline
\end{tabular}


Table 2. Adverse effects table (Continued) skin than patients treated with MAL-PDT.
The erysipelas and ulcer were treated with ambulant compression therapy and antibiotics. None of these patients needed hospitalisation.

\begin{tabular}{|c|c|c|}
\hline $\begin{array}{l}\text { Avril } 1997 \\
\text { (SE / ra- } \\
\text { diothera- } \\
\text { py) }\end{array}$ & Not stated. & $\begin{array}{l}\text { Main adverse characteristics } \\
\text { of surgical scars were "de- } \\
\text { formations" and "constric- } \\
\text { tions", affecting } 25 \% \text { and } 5 \% \\
\text { of patients respectively by } \\
\text { year } 4 \text {. Radiodystrophy and } \\
\text { necrosis affected } 41 \% \text { and } \\
5 \% \text { of the radiotherapy group } \\
\text { at } 4 \text { years respectively. }\end{array}$ \\
\hline
\end{tabular}

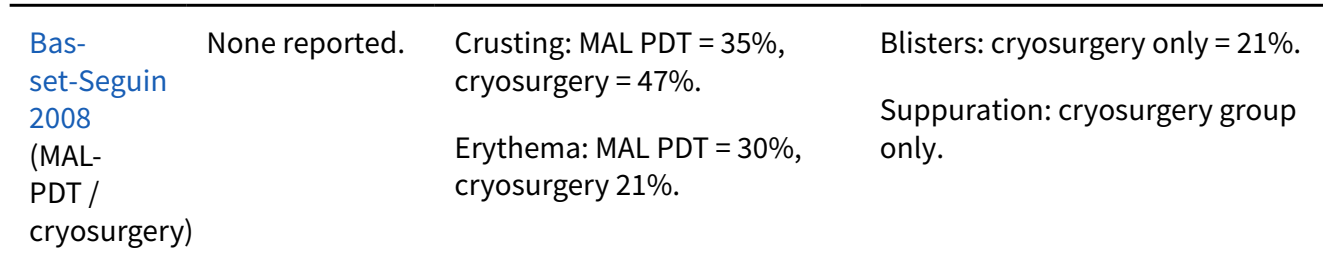

Three ophthalmic complications were observed: 1 ectropion, 1 cataract - after surgery. One lacrimal duct stenosis after radiotherapy.
Most were transient, resolv-
ing in 5 days.

More likely to experience severe AE in MAL-PDT group and moderate $A E$ in cryosurgery group, although most were mild $(73 \%$ and $80 \%$, respectively).

\begin{tabular}{|c|c|c|c|}
\hline $\begin{array}{l}\text { Bath-Hex- } \\
\text { tall } 2014 \\
\text { (Imiquimod / } \\
\text { SE) }\end{array}$ & $\begin{array}{l}12(5 \%) \text { partici- } \\
\text { pants in the im- } \\
\text { / iquimod group } \\
\text { withdrew be- } \\
\text { cause of ad- } \\
\text { verse effects } \\
\text { (five [ } 42 \%] \text { of } \\
\text { these events } \\
\text { were treatment } \\
\text { related). } \\
\text { Four ( } 2 \%) \text { of } \\
229 \text { partici- } \\
\text { pants withdrew } \\
\text { because of ad- } \\
\text { verse effects } \\
\text { in the surgery } \\
\text { group (all non- }\end{array}$ & $\begin{array}{l}\text { Itching: Imiquimod =211, SE } \\
=129 . \\
\text { Weeping: Imiquimod = } 160 \text {, } \\
\mathrm{SE}=81 .\end{array}$ & $\begin{array}{l}\text { Higher frequency adverse ef- } \\
\text { fects more common in imiquimod } \\
\text { group include 1) Mild/moderate } \\
\text { events: occurrence of new tu- } \\
\text { mours, redness and swelling at } \\
\text { tumour site, cold/influenza-like } \\
\text { symptoms, headache, scab at tu- } \\
\text { mour site, spots close to trial tu- } \\
\text { mour, discomfort and bleeding, 2) } \\
\text { Severe events: cold/flu, inflamma- } \\
\text { tory reactions. } \\
\text { Other high-frequency adverse ef- } \\
\text { fects, more common in SE group } \\
\text { include 1) Mild/moderate events: } \\
\text { Pain and swelling at tumour site } \\
\text { and 2) Severe events: Heat attack / } \\
\text { heart failure and pneumonia. }\end{array}$ \\
\hline
\end{tabular}

In the imiquimod group, 38 (15\%) participants needed a dose reduction.

No deaths or serious adverse effects were regarded as related to treatment.

$\begin{array}{ll}\text { Brinkhuizen } & \text { In } 16 \text { cases the } \\ 2016 \text { (di- } & \text { severity of the } \\ \text { clofenac / } & \text { application-site } \\ \text { diclofenac } & \text { reactions led to } \\ \text { and cal- } & \text { discontinuation } \\ \text { citriol / } & \text { of the therapy } \\ \text { calcitri- } & \text { and prescrip- } \\ \text { ol and } & \text { tion (diclofenac } \\ \text { no treat- } & =6, \text { calcitriol = } \\ \text { ment) } & 2, \text { combination } \\ & =8)\end{array}$

Brinkhuizen In 16 cases the

Erythema: diclofenac $=21$, calcitriol $=20$, combined treatment $=22$
Other commonly reported AEs in all groups include swelling, erosions, pruritus.

Other less frequent AEs include crust formation, vesicles, scaling, and paraesthesia.
Adverse effects were mostly mild to moderate.

No serious adverse effects were considered to be related to the study medication.

Three patients had serious adverse effects requiring hospitalisation.

No adverse effects were reported in the control group. 
Table 2. Adverse effects table (Continued)

$\begin{array}{ll}\text { Choi } 2016 \quad \text { None reported. } & \text { Crusting: Er-YAG AFL-PDT = } \\ \text { (Er:YAG } & 17, \text { MAL-PDT }=14 \\ \text { AFL MAL- } & \\ \text { PDT / } & \\ \text { MAL-PDT) } & \end{array}$

Erythema, burning sensation at application site, hyperpigmentation and itching more frequent in Er:YAG AFL-PDT group.

Other reactions include scale, bullae, oozing and bleeding.

\begin{tabular}{|c|c|c|}
\hline $\begin{array}{l}\text { Eigentler } \\
2007 \\
\text { (Imiquimod } \\
8 \text { weeks / } \\
12 \text { weeks) }\end{array}$ & None reported. & $\begin{array}{l}\text { Erythema and oedema oc- } \\
\text { curred in } 92 \% \text { of participants. } \\
\text { Reporting of vesicles, ero- } \\
\text { sions, ulcerations, desqua- } \\
\text { mation and drainage during } \\
\text { treatment was infrequent } \\
\text { (12-53\%). Post-treatment hy- } \\
\text { popigmentation was report- } \\
\text { ed in } 9 \% \text { of participants. Most } \\
\text { AEs were judged by physi- } \\
\text { cians as moderate severity } \\
\text { and by patients as moderate } \\
\text { (17\%) to severe (54\%). }\end{array}$ \\
\hline
\end{tabular}

\section{Authors reported "no differences} between treatment arms in adverse events".
All patients in both groups experienced some AEs.

\begin{tabular}{|c|c|c|c|}
\hline $\begin{array}{l}\text { Eimpunth } \\
2014 \\
\text { (Dou- } \\
\text { ble-stacked } \\
\text { PDL / con- } \\
\text { trol) }\end{array}$ & None reported. & $\begin{array}{l}\text { Purpura immediately after } \\
\text { laser treatment: } 100 \% \text { PDL } \\
\text { group. }\end{array}$ & $\begin{array}{l}\text { Late AEs in PDL group: blister } \\
(21.4 \%) \text {, dyspigmentation }(21.4 \%) \\
\text { and hypertrophic scar }(7.14 \%) \text {. }\end{array}$ \\
\hline
\end{tabular}

Data for AEs were not presented separately for treatment groups.

\begin{tabular}{|c|c|c|c|}
\hline $\begin{array}{l}\text { Ezughah } \\
2008 \\
\text { (Imiquimod } \\
8 \text { weeks / } \\
5 \text { weeks) }\end{array}$ & $\begin{array}{l}\text { One patient in } \\
5 \text { week group } \\
\text { withdrew from } \\
\text { the study due } \\
\text { to application } \\
\text { site reactions. }\end{array}$ & $\begin{array}{l}\text { Mild erythema (at week 1): } \\
\text { 8-week group = 69\%, } 5 \text { week } \\
\text { group }=69 \% \text {. }\end{array}$ & $\begin{array}{l}\text { Other reactions include pain, } \\
\text { swelling, weeping, broken skin and } \\
\text { scabbing. }\end{array}$ \\
\hline
\end{tabular}

Erythema decreased in both
groups at week 5 .

One patient on 8 week course developed severe erythema and soreness and was unable to apply $7^{\text {th }}$ treatment.

The severity of local site reactions was greatest in the first half of the treatment phase in the 8-week group.

\begin{tabular}{lll}
\hline Foley & 1 patient in & Burning: MAL-PDT = 19, \\
2009a/Fo- & MAL-PDT group & placebo $=8$. \\
ley 2009b & received only & \\
(MAL- & one treatment & Erythema: MAL-PDT = 14, \\
PDT / & due to local ad- & placebo $=4$. \\
placebo) & verse event. &
\end{tabular}

\begin{tabular}{lll}
\hline Gar- & None reported. & Blink discomfort (during \\
treatment): Imiquimod $=$ & Intense conjunctival irritation \\
cia-Mar- & $60 \%$, radiotherapy $=50 \%$. & fort 8.3\% radiotherapy group. \\
tin 2011 & & \\
(Imiquimod / & & \\
radiother- & & \\
apy) &
\end{tabular}

\author{
Other reactions include stinging of \\ skin, crusting and bleeding (more \\ frequent in treatment group). \\ Severe events: cholangiocarcino- \\ ma, cholelithiasis, carotid stenosis, \\ pulmonary oedema and acute MI,
melanoma, femoral artery surgery. \\ One serious AE deemed not \\ treatment-related. \\ All were mild-to-moderate \\ intensity and most lasted $<1$ \\ day.
}

After treatment, radiotherapy group reported ectropion, dry eye and loss of eyelashes. imiquimod group reported no post-treatment AEs. 
Table 2. Adverse effects table (Continued)

Geisse Thirteen pa2002 tients discon(Imiquimod tinued due to BD / OD / local skin re5xpw / actions, six of $3 x p w / v e-\quad$ which were sehicle) vere and four of which were due to adverse effects $(B D=1$, $\mathrm{OD}=2,5 \times \mathrm{xp}=$ $1)$.

Four discontinued dosing (3 because of pain, 1 of which was severe, and one because of bleeding).
Itching: $5 x p w=62 \%, O D=$ $61 \%, 3 \times p w=38 \%, B D=30 \%$, vehicle $=13 \%$.

Pain: $\mathrm{BD}=30 \%, \mathrm{OD}=16 \%$, $3 \mathrm{xpw}=10 \%, 5 \mathrm{xpw}=8 \%$, vehicle $=0$.

Tenderness: $\mathrm{BD}=20 \%, \mathrm{OD}=$ $16 \%, 5 \times p w=8 \%, 3 \times p w=7 \%$, vehicle $=6 \%$
Other reactions include erythema, scabbing, erosion, excoriation/flaking, ulceration, vesicles, induration and oedema.

Severe erythema and scabbing occurred more commonly in the twice-daily treatment groups.
AEs occurred in all treatment groups, as did local skin reactions (all of those deemed related to study drug were in the OD group).

Six serious AEs, all unrelated to treatment, five of which resulted in hospitalisation.

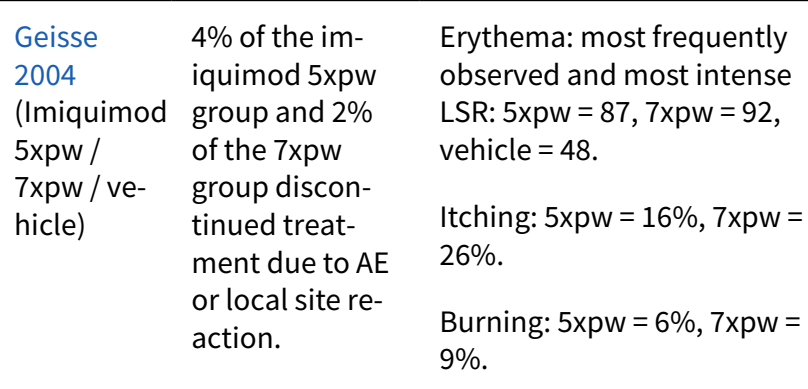

Other reactions include oedema, induration, vesicle, erosion, ulceration, scabbing and flaking.

The incidence of headache was statistically higher in the Imiquimod 5xpw group compared to the corresponding vehicle group $(P=0.027)$, although this difference was not seen in the $7 x p w$ group.
Local site reactions reached maximum intensity at week 3 , and most were mild.

Local site reactions were experienced in all groups but more likely in imiquimod groups, and these were statistically more likely to be severe $(P=0.001)$.

AEs occurred more frequently during treatment than after and were more likely to occur in the imiquimod 7xpw group than the 5xpw group or the vehicle groups $(64 \%$ compared to $58 \%$ and $36 \%$ (combined vehicle groups).

Local site reactions were experienced more commonly in 7xpw Imiquimod group compared to the 5xpw group $(\mathrm{P}=$ 0.002).

AEs considered possibly or probably related to treatment include local site reactions and lymphadenopathy in the $7 x p w$ Imiquimod group.

All local site reactions were significantly more likely to be intense than 7xpw group than $5 x p w$ group $(P<0.5)$. 
Overall the severe AEs experience by participants were in the $7 x p w$ Imiquimod group.

A decrease in WCC and neutrophils was noted in the treatment groups.

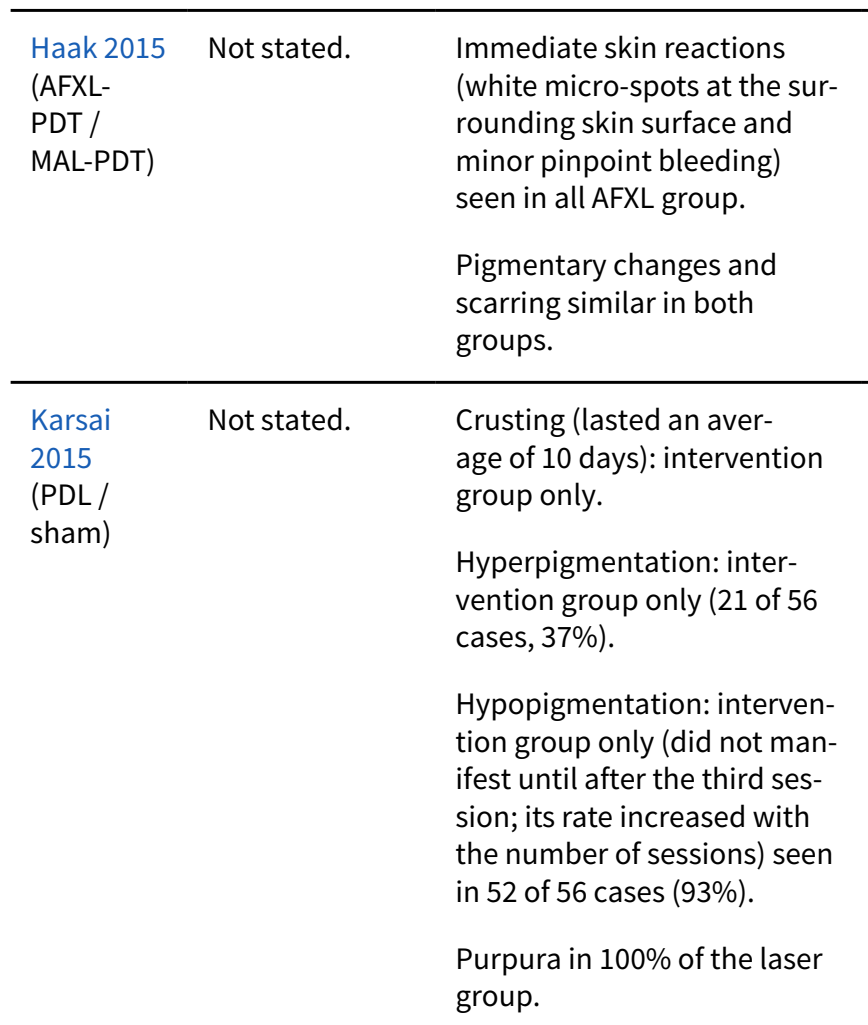

\begin{tabular}{|c|c|c|c|c|}
\hline $\begin{array}{l}\text { Kessels } \\
2017 \\
\text { (Sinecat- } \\
\text { echins / } \\
\text { placebo) }\end{array}$ & Not stated. & $\begin{array}{l}\text { Erythema: sinecatechins } \\
\text { 9-12\%, placebo 2-6\%. } \\
\text { Oedema: sinecatechins 2-6\%, } \\
\text { placebo } 0 \% \text {. } \\
\text { Erosions: sinecatechins 4-7\%, } \\
\text { placebo } 1 \% \text {. } \\
\text { Crusts: sinecatechins 3-10\%, } \\
\text { placebo } 2 \% \text {. } \\
\text { Itching: sinecatechins } 10-13 \\
\% \text {, placebo } 1-3 \% \text {. }\end{array}$ & $\begin{array}{l}\text { Other reactions include oedema, } \\
\text { erosions, bullae and squamae. }\end{array}$ & $\begin{array}{l}\text { Statistically significant differ- } \\
\text { ence for all common AEs. }\end{array}$ \\
\hline $\begin{array}{l}\text { Kessels } \\
2018 \\
\text { (MAL- } \\
\text { PDT / two- } \\
\text { fold frac- } \\
\text { tionated } \\
\text { ALA-PDT) }\end{array}$ & Not stated. & $\begin{array}{l}\text { Erythema: } \text { MAL-PDT }=65 \text {, } \\
\text { ALA-PDT }=73 . \\
\text { Wounds/erosions: MAL-PDT = } \\
64, \text { ALA-PDT }=72 \text {. } \\
\text { Vesicles: MAL-PDT = 66, ALA- } \\
\text { PDT = } 72 \text {. } \\
\text { All significantly higher after } \\
\text { ALA-PDT compared with MAL- } \\
\text { PDT. }\end{array}$ & $\begin{array}{l}\text { Other reactions include swelling, } \\
\text { crusts, scaling and pruritus. } \\
\text { Four serious AEs unrelated to } \\
\text { study treatment (three hospitaliza- } \\
\text { tions owing to transient ischaemic } \\
\text { attack, chemotherapy for lung car- } \\
\text { cinoma and dizziness, and one pa- } \\
\text { tient died owing to cancer). }\end{array}$ & $\begin{array}{l}\mathrm{AE} \text { incidence higher during } \\
\text { treatment period than after } \\
\text { treatment. }\end{array}$ \\
\hline
\end{tabular}

Minor bleeding and bruising in debulked areas.

Not stated.

Scarring was the most frequently observed reaction at 3 months.

\author{
Purpura was considered a \\ desired concomitant effect \\ and was seen for an average \\ of 6 days.
}

Hyper- and hypopigmentation persisted until the end of the final follow-up at 6 months. 
Table 2. Adverse effects table

(Continued)

No serious unexpected adverse reactions were reported in either group.

\begin{tabular}{|c|c|c|}
\hline $\begin{array}{l}\text { Kuijpers } \\
2007 \text { (SE / } \\
\text { curet- } \\
\text { tage and } \\
\text { cryosurgery) }\end{array}$ & Not stated. & $\begin{array}{l}\text { Secondary wound infec- } \\
\text { tion requiring systemic an- } \\
\text { tibiotics: curettage and } \\
\text { cryosurgery group = } 3 \text { pa- } \\
\text { tients, SE group = } 4 \text { patients. }\end{array}$ \\
\hline
\end{tabular}

\begin{tabular}{|c|c|c|c|c|}
\hline $\begin{array}{l}\text { Marks } \\
2001 \\
\text { (Imiquimod } \\
\text { BD / OD / } \\
\text { BD for } 3 \\
\text { days / OD } \\
\text { for } 2 \text { days) }\end{array}$ & $\begin{array}{l}\text { One patient dis- } \\
\text { continued be- } \\
\text { cause of a self- } \\
\text { reported ap- } \\
\text { plication site } \\
\text { symptom of } \\
\text { pruritus. } \\
\text { No patient } \\
\text { withdrew from } \\
\text { the study be- } \\
\text { cause of signs }\end{array}$ & $\begin{array}{l}\text { Erythema: } \mathrm{BD}=66.7 \%, \mathrm{OD}= \\
27.3 \%, \mathrm{BD} 3 \mathrm{pw},=13.3 \%, \mathrm{OD} \\
\text { x3pw }=9.1 \% . \\
\text { Itching: } \mathrm{BD}=33.3 \%, \mathrm{OD}= \\
66.7 \%, \mathrm{BD} \times 3 \mathrm{pw},=36.7 \%, \mathrm{OD} \\
\text { x3pw }=45.5 \% \text {. } \\
\text { Weeping: decreased as the } \\
\text { dosing frequency decreased } \\
\text { BD }=66.7 \% \text { OD }=12.1 \% \\
\text { BDx3pw, }=3.3 \%, \text { OD } \times 3 \mathrm{pw}= \\
0 \% \text {. }\end{array}$ & $\begin{array}{l}\text { Other reactions include scabbing, } \\
\text { flaking, erosion, ulceration, oede- } \\
\text { ma and induration. } \\
\text { One patient died as the result of a } \\
\text { coincidental cerebrovascular acci- } \\
\text { dent. }\end{array}$ & $\begin{array}{l}\text { Local skin reactions occurred } \\
\text { in all } 4 \text { dose regimens; dose- } \\
\text { dependent application site } \\
\text { reactions being reported } \\
\text { most often. } \\
\text { In the twice-every-day group, } \\
\text { all the local skin reactions } \\
\text { were assessed as severe by } \\
\text { both the patient and the in- } \\
\text { vestigator in two thirds of the } \\
\text { patients. }\end{array}$ \\
\hline
\end{tabular}
of a local skin reaction assessed by the investigators.

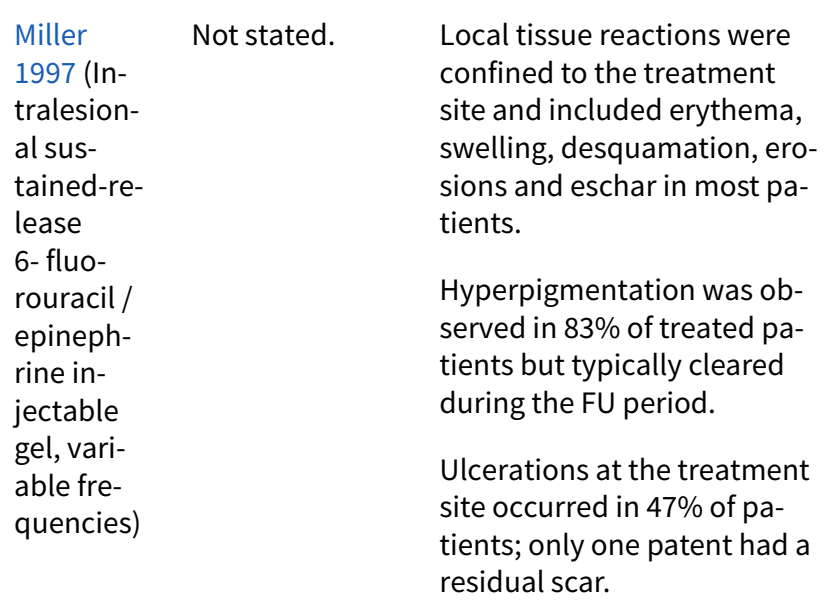

Not stated.

There were no clinically significant serious or unexpected adverse effects or changes in any lab values or physical examination findings as judged by the investigator to be related to the administration of 5-FU/epi gel. The number of events per regimen ranged from 17 to 28 .

\begin{tabular}{|c|c|c|c|}
\hline $\begin{array}{l}\text { Morton } \\
2018 \text { (BF } \\
200 \text { ALA- } \\
\text { PDT / } \\
\text { MAL-PDT) }\end{array}$ & $\begin{array}{l}\text { One patient } \\
\text { withdrew } \\
\text { due to treat- } \\
\text { ment-related } \\
\text { AEs in the BF } \\
200 \text { ALA group } \\
\text { and } 2 \text { in the } \\
\text { MAL group. } \\
\text { Two other pa- } \\
\text { tients withdrew } \\
\text { due to relat- } \\
\text { ed treatment } \\
\text { emergent ad- } \\
\text { verse effects }\end{array}$ & $\begin{array}{l}\text { Most frequently reported re- } \\
\text { actions included pain, ery- } \\
\text { thema, pruritus and oedema; } \\
\text { most frequently at mild to } \\
\text { moderate intensity. } \\
\text { Frequencies were compara- } \\
\text { ble between the groups and } \\
\text { revealed no statistically sig- } \\
\text { nificant differences. }\end{array}$ & $\begin{array}{l}\text { There were ten serious } A E s \text {, none } \\
\text { of which were related to the study } \\
\text { medication. }\end{array}$ \\
\hline
\end{tabular}


Table 2. Adverse effects table (Continued)

(one in each

group).

\begin{tabular}{ll}
\hline Mosterd None reported. Not stated. & Not
\end{tabular}

PDT / SE)

Secondary wound infection was

observed once after ALA-PDT treat-

ment. No serious complications

such as wound dehiscence or

necrosis were observed in either

ALA-PDT or SE group.

\begin{tabular}{ll}
\hline Punjabi & 6 patients with- \\
2008 (So- & drew due to \\
lasodine & severity of lo- \\
glycoside & cal irritation in \\
cream / & the treatment \\
vehicle) & group.
\end{tabular}

There were four serious adverse

effects in treatment group ( 3 un-

related, 1 unlikely to be related to

treatment), and 2 in the vehicle

group (one unrelated and one un-

likely related to treatment).
No SAEs were considered likely or related to the treatment and there was no significant difference between the two groups.

\begin{tabular}{|c|c|c|c|c|}
\hline $\begin{array}{l}\text { Rhodes } \\
2004 \\
\text { (MAL- } \\
\text { PDT / SE) }\end{array}$ & $\begin{array}{l}\text { One discontin- } \\
\text { uation due to } \\
\text { adverse vent in } \\
\text { MAL-PDT group. }\end{array}$ & $\begin{array}{l}\text { Erythema: } M A L-P D T=7, S E= \\
1 . \\
\text { Skin infection: } M A L-P D T=0, \\
\text { SE }=3 .\end{array}$ & $\begin{array}{l}\text { Three patients had skin infections } \\
\text { after SE (none in PDT group). } \\
\text { Other local adverse effects includ- } \\
\text { ed crusting and itching. } \\
\text { There were three deaths, all un- } \\
\text { related to treatment, and one pa- } \\
\text { tient was diagnosed with breast } \\
\text { cancer during treatment. }\end{array}$ & $\begin{array}{l}\text { More patients with MAL-PDT } \\
\text { than SE experienced adverse } \\
\text { effects ( } 52 \% \text { compared to } \\
29 \% \text { ). }\end{array}$ \\
\hline
\end{tabular}

\begin{tabular}{|c|c|c|}
\hline $\begin{array}{l}\text { Ro- } \\
\text { magosa } \\
2000 \text { (5- } \\
\text { FU in PC } \\
\text { vehicle / } \\
5-F U \text { in } \\
\text { petrola- } \\
\text { tum base) }\end{array}$ & Not stated. & $\begin{array}{l}\text { Local irritation, erythema, } \\
\text { ulceration, tenderness were } \\
\text { common but well-tolerated. }\end{array}$ \\
\hline
\end{tabular}

Minimal itching in both treatment arms.

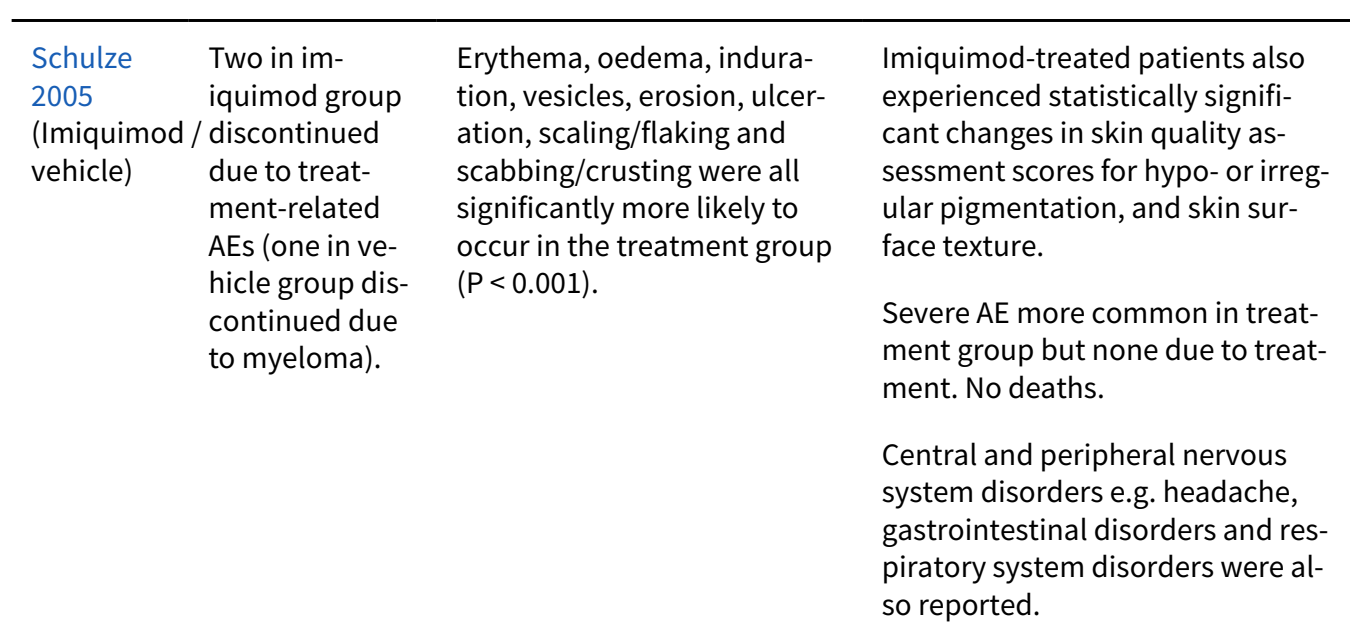

\begin{tabular}{|c|c|c|}
\hline $\begin{array}{l}\text { Shumack } \\
2002 a \text { (im- } \\
\text { iquimod, } \\
\text { several } \\
\text { regimes } \\
\text { for } 6 \\
\text { weeks) }\end{array}$ & $\begin{array}{l}\text { One patient dis- } \\
\text { continued due } \\
\text { to treatment } \\
\text { related side ef- } \\
\text { fects (OD } 7 \text { day } \\
\text { dosing group), } \\
\text { one discon- }\end{array}$ & $\begin{array}{l}\text { Erythema and scabbing com- } \\
\text { monly reported. }\end{array}$ \\
\hline
\end{tabular}

Local skin reactions were more intense in the imiquimod group (statistically significant).

Local skin reactions increased at week 2 and plateaued until week 6 . 
Table 2. Adverse effects table (Continued)

tinued due to

cerebral vascu-

lar accident.

\begin{tabular}{ll}
\hline $\begin{array}{l}\text { Shumack } \\
\text { 2002b }\end{array}$ & 8 patients dis- \\
(im- & continued \\
iquimod, & treatment ow- \\
ing to local skin \\
regimes & reaction (2 in \\
for 12 & OD 5 day group, \\
weeks) & 4 in OD 7 day \\
& group, 2 in BD 7 \\
& day group). \\
& 2 discontin- \\
& ued due to AEs \\
& (1= pain and \\
& drainage at tar- \\
& get site and $1=$ \\
& spider bite ad- \\
& jacent to target \\
& site).
\end{tabular}

Siller 2010 Two discontin-

(ingenol

mebu-

tate gel

$0.0025 \%$ /

ingenol

mebu-

tate gel

$0.01 \%$ /

ingenol

mebu-

tate gel

$0.05 \%$ /

vehicle)
Erythema and scabbing commonly reported.

The most common reactions were erythema and flaking/scaling/dryness, which occurred in a minimum of 75 and $50 \%$ respectively, of all patients in all groups (including vehicle group).
Hyper- and hypopigmentation, erosion/ulceration, oedema, scabbing/crusting, vesicles all more common in ingenol mebutate gel $0.05 \%$ group. Most were mild to moderate reactions, although 2 were severe (ingenol mebutate gel 0.01 and $0.05 \%$ respectively).

Other skin reactions include itch, weeping/exudate, scarring.

Other adverse effects include liver function derangement, diarrhoea, erythema, flaking, headache, oedema, pustules at application site, pustular rash, scabbing and telangiectasia.

33 patients died due to conditions not related to the treatment.
Local skin reactions occurred in all study groups, and were the most commonly reported $A E$ (most mild/moderate intensity).
No serious $A E$.

Severe AE included mild/mod erythema extending beyond treatment site and headache.

10 severe local skin reactions seen in Arm A in ingenol mebutate gel $0.01 \%$ / ingenol mebutate gel $0.05 \%$ groups.

\begin{tabular}{|c|c|c|c|c|}
\hline $\begin{array}{l}\text { Smeets } \\
2004 \\
\text { (MMS / SE) }\end{array}$ & Not stated. & $\begin{array}{l}\text { AEs in SE group include } \\
\text { wound infection, necrosis of } \\
\text { grafts or flaps or post-opera- } \\
\text { tive bleeding. }\end{array}$ & $\begin{array}{l}33 \text { patients died due to conditions } \\
\text { not related to the treatment. }\end{array}$ & $\begin{array}{l}12 \% \text { complications in MMS } \\
\text { group and } 14 \% \text { in SE group ( } P \\
=0.981) .\end{array}$ \\
\hline $\begin{array}{l}\text { Soler } 2000 \\
\text { (laser } \\
\text { light / } \\
\text { broad- } \\
\text { band } \\
\text { light) }\end{array}$ & Not stated. & $\begin{array}{l}\text { During the 1st week after } \\
\text { treatment, } 68 \% \text { patients } \\
\text { in the laser group and } 74 \% \\
\text { of those in the broadband } \\
\text { group also reported some } \\
\text { degree of discomfort (sting- } \\
\text { ing, itching, pain, suppura- } \\
\text { tion headache, sensation of } \\
\text { warmth or blushing). }\end{array}$ & Not stated. & $\begin{array}{l}\text { No serious AEs reported in } \\
\text { the 6-month follow-up peri- } \\
\text { od. } \\
\text { There was no statistically sig- } \\
\text { nificant difference between } \\
\text { the groups. }\end{array}$ \\
\hline $\begin{array}{l}\text { Spel- } \\
\text { man } 2014 \\
\text { (Ingenol } \\
\text { mebutate }\end{array}$ & $\begin{array}{l}81.5 \% \text { toler- } \\
\text { ated only one } \\
\text { treatment in } \\
\text { full-ODr group, }\end{array}$ & Not stated. & $\begin{array}{l}\text { Local skin reactions in } 11.3 \text { full ODr } \\
\text { group, } 7,5 \text { in semi-ODr group and } \\
8.9 \text { in no-ODr group. }\end{array}$ & $\begin{array}{l}\text { The majority of AEs were not } \\
\text { considered treatment relat- } \\
\text { ed. The percentage of AEs } \\
\text { was similar across all treat- }\end{array}$ \\
\hline
\end{tabular}


Table 2. Adverse effects table (Continued)

ferent oc- $\quad 20.8 \%$ in se-

clusion mi-ODrgroup

types: full and $4.2 \%$ in

ODr, Se- non-ODr group.

mi-ODr or

no-ODr) under dif- compared to

ment groups, the most common being application site pain. The majority of treatment-related AEs were in the ALU group, and in this group the majority only tolerated one treatment due to the severity of the local skin reaction.

\begin{tabular}{|c|c|c|c|c|}
\hline $\begin{array}{l}\text { Sterry } \\
2002 a, \\
\text { sBCC } \\
\text { study } \\
\text { (Imiquimod } \\
\text { with / } \\
\text { without } \\
\text { occlusion, } \\
\text { both } 2 \text { or } \\
3 \text { days per } \\
\text { week) }\end{array}$ & $\begin{array}{l}\text { Two patients } \\
\text { discontinued } \\
\text { from the study, } \\
\text { one due to an } \\
\text { adverse event } \\
\text { (neurologi- } \\
\text { cal dysregula- } \\
\text { tion; not treat- } \\
\text { ment-related) } \\
\text { and one due to } \\
\text { a local skin re- } \\
\text { action. One pa- } \\
\text { tient in with- } \\
\text { out-occlusion } \\
\text { group required } \\
\text { interrupted } \\
\text { dosing and two } \\
\text { required rest } \\
\text { periods ( } 3 x p w \\
\text { with occlusion } \\
\text { and 2xpw with- } \\
\text { out occlusion). }\end{array}$ & $\begin{array}{l}\text { Itching, burning and hy- } \\
\text { popigmentation most fre- } \\
\text { quently reported AEs. }\end{array}$ & $\begin{array}{l}\text { Two severe application site reac- } \\
\text { tions were considered due to the } \\
\text { medication (in } 2 \times p w \text { without oc- } \\
\text { clusion group and in } 3 x p w \text { with oc- } \\
\text { clusion group). } \\
\text { Fever and influenza-like symptoms } \\
\text { were infrequently reported. } \\
\text { Four patients experienced serious } \\
\text { adverse effects unrelated to the } \\
\text { medication. }\end{array}$ & $\begin{array}{l}\text { AEs occurred in all treatment } \\
\text { groups with } 59 \% \text { reporting at } \\
\text { least one. } \\
32 \% \text { reported application site } \\
\text { reactions, which occurred } \\
\text { more often in groups treated } \\
\text { three times per week. }\end{array}$ \\
\hline $\begin{array}{l}\text { Sterry } \\
2002 \text { b, } \\
\text { nBCC } \\
\text { study } \\
\text { (Imiquimod } \\
\text { with / } \\
\text { without } \\
\text { occlusion, } \\
\text { both } 2 \text { or } \\
3 \text { days per } \\
\text { week) }\end{array}$ & $\begin{array}{l}\text { Two discontin- } \\
\text { ued due to local } \\
\text { skin reactions } \\
\text { (severe erythe- } \\
\text { ma, oedema } \\
\text { and crust) and } \\
\text { three discontin- } \\
\text { ued due to ap- } \\
\text { plication site } \\
\text { reactions. Rest } \\
\text { periods were } \\
\text { taken by three } \\
\text { patients. }\end{array}$ & $\begin{array}{l}\text { Bleeding, itching and irrita- } \\
\text { tion most frequently report- } \\
\text { ed AEs. } \\
\text { Erythema was the most fre- } \\
\text { quently reported local skin } \\
\text { reaction and was most com- } \\
\text { mon in the 3xpw group. }\end{array}$ & $\begin{array}{l}\text { Five experienced severe appli- } \\
\text { cation site reactions considered } \\
\text { probably related to the study drug. } \\
\text { In groups receiving } 3 x p w \text { treat- } \\
\text { ment, severe local skin reactions } \\
\text { were most common. } \\
\text { Four experienced severe AEs unre- } \\
\text { lated to study drug. }\end{array}$ & $\begin{array}{l}70 \% \text { reported adverse ef- } \\
\text { fects. } 42 \% \text { reported appli- } \\
\text { cation site reactions; local } \\
\text { skin reactions occurred in all } \\
\text { treatment groups, most fre- } \\
\text { quently in the } 3 x p w \text { occlu- } \\
\text { sion group. }\end{array}$ \\
\hline $\begin{array}{l}\text { Szeimies } \\
2008 \\
\text { (MAL- } \\
\text { PDT / SE) }\end{array}$ & $\begin{array}{l}2 \text { patients in } \\
\text { MAL-PDT group } \\
\text { and one in SE } \\
\text { group (none } \\
\text { treatment re- } \\
\text { lated). }\end{array}$ & $\begin{array}{l}\text { Photosensitivity reactions: } \\
\text { MAL-PDT }=37 \%, \text { SE }=0 \% \text {. } \\
\text { Wound infection: MAL-PDT = } \\
0 \%, \text { SE }=5.2 \% \text {. }\end{array}$ & $\begin{array}{l}\text { Other reported AEs included ery- } \\
\text { thema, post-procedural pain, milia } \\
\text { and wound dehiscence. } \\
21 \text { serious AEs were reported; none } \\
\text { were considered related and any } \\
\text { permanent discontinuations were } \\
\text { unrelated to treatment. }\end{array}$ & $\begin{array}{l}\text { Treatment-related AEs were } \\
\text { higher in MAL-PDT group } \\
\text { than in SE group ( } 37 \% \text { versus } \\
14.6 \% \text { ). } \\
\text { All AEs were mild to moder- } \\
\text { ate severity except one se- } \\
\text { vere wound infection in SE } \\
\text { group. }\end{array}$ \\
\hline
\end{tabular}

Not stated. ticipants developed eschars at 2 weeks, followed by mild 
Table 2. Adverse effects table (Continued)

gle puls-

es/PDL

erythema at 1 month and hy-

$10 \mathrm{~mm}$

stacked

pulses)

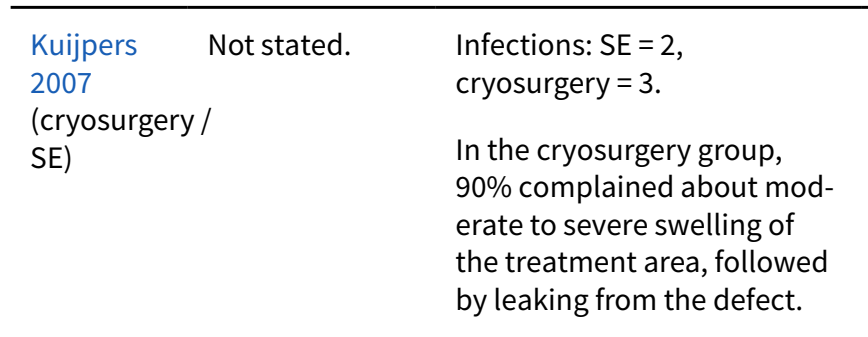

Wang Not stated. Not stated.
2001 (ALA-
PDT /
cryosurgery)

One death (unrelated to study tumour). popigmentation at 5 months.

cryosurgery)

\begin{tabular}{|c|c|c|}
\hline $\begin{array}{l}2005-001474-\text { Not stated. } \\
\text { (place- } \\
\text { bo gel + } \\
\text { Tazarotene } \\
\text { gel / Bace- } \\
\mathrm{ca}^{\boxplus}+ \\
\text { Tazarotene } \\
\text { gel) }\end{array}$ & $\begin{array}{l}\text { Erythema ( } 17 / 25 \text { and } 20 / 25 \text { in } \\
\text { the two treatment groups). }\end{array}$ & $\begin{array}{l}\text { Itch ( } 18 \text { versus } 15 \text { patients), ulcera- } \\
\text { tion ( } 11 \text { versus } 16 \text { patients) and lo- } \\
\text { cal pain ( } 8 \text { versus } 6 \text { patients). }\end{array}$ \\
\hline
\end{tabular}

\begin{abstract}
There were two patient deaths, and both were unrelated to the $\mathrm{BCC}$ or the treatment.

One patient reported pain radiating from treatment site (PDT group) and one developed bacterial infection cryosurgery group).
\end{abstract}

No significant differences between the two groups. Extension phase outcomes not reported.

MAL: methyl aminolevulinate; PDL: pulsed dye laser; PDT: photodynamic therapy; SE: surgical excision; xpw: times per week.

Table 3. Curettage and cryosurgery compared to surgical excision for basal cell carcinoma of the skin

\section{Curettage \& cryosurgery compared to surgical excision for low-risk BCC}

Patient or population: adults with basal cell carcinoma of the skin

Setting: secondary care with outpatients from a single-centre in The Netherlands

Intervention: curettage \& cryosurgery

Comparison: surgical excision

\begin{tabular}{|c|c|c|c|c|c|c|}
\hline \multirow[t]{2}{*}{ Outcomes } & \multicolumn{2}{|c|}{ Anticipated absolute effects ${ }^{\star}(95 \% \mathrm{CI})$} & \multirow{2}{*}{$\begin{array}{l}\text { Relative effect } \\
(95 \% \mathrm{Cl})\end{array}$} & \multirow{2}{*}{$\begin{array}{l}\text { № of } \\
\text { partici- } \\
\text { pants } \\
\text { (stud- } \\
\text { ies) }\end{array}$} & \multirow{2}{*}{$\begin{array}{l}\text { Certain- } \\
\text { ty of } \\
\text { the evi- } \\
\text { dence } \\
\text { (GRADE) }\end{array}$} & \multirow[t]{2}{*}{ Comments } \\
\hline & $\begin{array}{l}\text { Risk with surgical } \\
\text { excision }\end{array}$ & $\begin{array}{l}\text { Risk with Curettage \& } \\
\text { cryosurgery }\end{array}$ & & & & \\
\hline \multirow{2}{*}{$\begin{array}{l}\text { Recurrence at } 3 \\
\text { years }\end{array}$} & Study population & & \multirow{2}{*}{$\begin{array}{l}\text { RR } 3.36 \\
(0.73 \text { to } 15.40)\end{array}$} & \multirow{2}{*}{$\begin{array}{l}100 \\
(1 \mathrm{RCT})\end{array}$} & \multirow{2}{*}{$\begin{array}{l}\oplus \oplus \ominus \ominus \\
\text { LOW } 1\end{array}$} & \multirow[t]{2}{*}{-} \\
\hline & 41 per 1000 & $\begin{array}{l}137 \text { per } 1000 \\
\text { (30 to } 629)\end{array}$ & & & & \\
\hline \multirow{2}{*}{$\begin{array}{l}\text { Recurrence at } 5 \\
\text { years }\end{array}$} & Study population & & \multirow{2}{*}{$\begin{array}{l}\text { RR } 2.78 \\
\text { (0.93 to 8.34) }\end{array}$} & \multirow{2}{*}{$\begin{array}{l}85 \\
(1 \mathrm{RCT})\end{array}$} & \multirow{2}{*}{$\begin{array}{l}\oplus \oplus \oplus \Theta \\
\text { MODER- } \\
\text { ATE } 2\end{array}$} & \multirow[t]{2}{*}{-} \\
\hline & 85 per 1000 & 237 per 1000 & & & & \\
\hline
\end{tabular}


Table 3. Curettage and cryosurgery compared to surgical excision for basal cell carcinoma of the skin (Continued) (79 to 710$)$

\begin{tabular}{|c|c|c|c|c|c|c|}
\hline \multirow{2}{*}{$\begin{array}{l}\text { Cosmetic out- } \\
\text { come (good/excel- } \\
\text { lent) }\end{array}$} & \multicolumn{2}{|c|}{ Study population } & \multirow{2}{*}{$\begin{array}{l}\text { RR } 0.88 \\
\text { (0.78 to } 0.98)\end{array}$} & \multirow{2}{*}{$\begin{array}{l}96 \\
(1 \mathrm{RCT})\end{array}$} & \multirow{2}{*}{$\begin{array}{l}\oplus \oplus \ominus \ominus \\
\text { LOW }^{3}\end{array}$} & \multirow{2}{*}{$\begin{array}{l}\text { Partici- } \\
\text { pant-rated at } \\
1 \text { year on a 3- } \\
\text { point scale. }{ }^{4}\end{array}$} \\
\hline & 1000 per 1000 & $\begin{array}{l}880 \text { per } 1000 \\
(780 \text { to } 980)\end{array}$ & & & & \\
\hline \multirow{2}{*}{$\begin{array}{l}\text { Cosmetic out- } \\
\text { come (good/excel- } \\
\text { lent) }\end{array}$} & Study populat & & \multirow{2}{*}{$\begin{array}{l}\text { RR } 0.28 \\
\text { (0.16 to } 0.47 \text { ) }\end{array}$} & \multirow{2}{*}{$\begin{array}{l}96 \\
(1 \mathrm{RCT})\end{array}$} & \multirow{2}{*}{$\begin{array}{l}\oplus \oplus \ominus \ominus \\
\text { LOW } 3\end{array}$} & \multirow{2}{*}{$\begin{array}{l}\text { Observ- } \\
\text { er-rated at } 1 \\
\text { year on a 3- } \\
\text { point scale. }{ }^{4}\end{array}$} \\
\hline & 833 per 1000 & $\begin{array}{l}233 \text { per } 1000 \\
\text { (133 to } 392 \text { ) }\end{array}$ & & & & \\
\hline
\end{tabular}

\begin{tabular}{l}
$\begin{array}{l}\text { Early treatment } \quad \text { No study addressed this outcome } \\
\text { failure }\end{array} \quad$ not estimable not estimable \\
\hline Pain $\quad$ No study addressed this outcome \\
$\begin{array}{l}{ }^{*} \text { The risk in the intervention group (and its } 95 \% \text { confidence interval) is based on the assumed risk in the comparison group and the } \\
\text { relative effect of the intervention (and its } 95 \% \mathrm{Cl}) .\end{array}$ \\
Cl: Confidence interval; RR: Risk ratio.
\end{tabular}

\section{GRADE Working Group grades of evidence}

High certainty: we are very confident that the true effect lies close to that of the estimate of the effect.

Moderate certainty: we are moderately confident in the effect estimate; the true effect is likely to be close to the estimate of the effect, but there is a possibility that it is substantially different.

Low certainty: our confidence in the effect estimate is limited; the true effect may be substantially different from the estimate of the effect.

Very low certainty: we have very little confidence in the effect estimate; the true effect is likely to be substantially different from the estimate of effect.

1 Downgraded two levels for very serious imprecision as very wide $95 \% \mathrm{Cl}$ indicating the possibility of important benefit or harm.

2 Downgraded one level for serious imprecision as only a single study with a small sample size and a wide $95 \% \mathrm{Cl}$ and downgraded one level for serious risk of bias as unable to truly blind due to the nature of interventions.

3 Downgraded one level for serious imprecision as only a single study with a small sample size.

4 3-point scale: bad, fair, good.

Table 4. ALA-PDT compared to surgical excision for basal cell carcinoma of the skin

\section{ALA-PDT compared to surgical excision for low-risk BCC}

Patient or population: adults with basal cell carcinoma of the skin

Setting: secondary care with outpatients from a single-centre in the Netherlands

Intervention: ALA-PDT

Comparison: surgical excision

\begin{tabular}{|c|c|c|c|c|c|c|}
\hline \multirow[t]{2}{*}{ Outcomes } & \multicolumn{2}{|c|}{ Anticipated absolute effects* $(95 \% \mathrm{Cl})$} & \multirow{2}{*}{$\begin{array}{l}\text { Relative effect } \\
(95 \% \mathrm{Cl})\end{array}$} & \multirow{2}{*}{$\begin{array}{l}\text { № of } \\
\text { partici- } \\
\text { pants } \\
\text { (stud- } \\
\text { ies) }\end{array}$} & \multirow{2}{*}{$\begin{array}{l}\text { Certainty } \\
\text { of the evi- } \\
\text { dence } \\
\text { (GRADE) }\end{array}$} & \multirow{2}{*}{$\begin{array}{l}\text { Com- } \\
\text { ments }\end{array}$} \\
\hline & $\begin{array}{l}\text { Risk with surgical ex- } \\
\text { cision }\end{array}$ & Risk with ALA-PDT & & & & \\
\hline \multirow{2}{*}{$\begin{array}{l}\text { Recurrence at } 3 \\
\text { years }\end{array}$} & Study population & & \multirow{2}{*}{$\begin{array}{l}\text { RR } 10.87 \\
\text { (2.63 to } 44.95)\end{array}$} & \multirow{2}{*}{$\begin{array}{l}173 \\
(1 \mathrm{RCT})\end{array}$} & \multirow{2}{*}{$\begin{array}{l}\oplus \oplus \oplus \ominus \\
\text { MODERATE } \\
1\end{array}$} & \multirow[t]{2}{*}{-} \\
\hline & 23 per 1000 & $\begin{array}{l}247 \text { per } 1000 \\
(60 \text { to } 1000)\end{array}$ & & & & \\
\hline
\end{tabular}


Table 4. ALA-PDT compared to surgical excision for basal cell carcinoma of the skin (Continued)

\begin{tabular}{|c|c|c|c|c|c|c|}
\hline \multirow{2}{*}{$\begin{array}{l}\text { Recurrence at } 5 \\
\text { years }\end{array}$} & \multicolumn{2}{|c|}{ Study population } & \multirow{2}{*}{$\begin{array}{l}\text { RR } 11.91 \\
\text { (2.90 to } 48.95 \text { ) }\end{array}$} & \multirow{2}{*}{$\begin{array}{l}173 \\
(1 \mathrm{RCT})\end{array}$} & \multirow{2}{*}{$\begin{array}{l}\oplus \oplus \oplus \ominus \\
\text { MODERATE } \\
1\end{array}$} & \multirow[t]{2}{*}{-} \\
\hline & 23 per 1000 & $\begin{array}{l}271 \text { per } 1000 \\
(66 \text { to } 1000)\end{array}$ & & & & \\
\hline $\begin{array}{l}\text { Cosmetic out- } \\
\text { come }\end{array}$ & \multicolumn{2}{|c|}{ No study addressed this outcome. } & not estimable & - & - & - \\
\hline Pain & \multicolumn{2}{|c|}{ No study addressed this outcome. } & not estimable & - & - & - \\
\hline \multirow{2}{*}{$\begin{array}{l}\text { Early treatment } \\
\text { failure }\end{array}$} & \multicolumn{2}{|c|}{ Study population } & RR 3.18 & 171 & $\oplus \oplus \ominus \ominus$ & - \\
\hline & 23 per 1000 & $\begin{array}{l}72 \text { per } 1000 \\
(15 \text { to } 348)\end{array}$ & & & & \\
\hline
\end{tabular}

${ }^{*}$ The risk in the intervention group (and its $95 \%$ confidence interval) is based on the assumed risk in the comparison group and the relative effect of the intervention (and its $95 \% \mathrm{Cl}$ ).

Cl: Confidence interval; RR: Risk ratio.

\section{GRADE Working Group grades of evidence}

High certainty: we are very confident that the true effect lies close to that of the estimate of the effect.

Moderate certainty: we are moderately confident in the effect estimate; the true effect is likely to be close to the estimate of the effect, but there is a possibility that it is substantially different.

Low certainty: our confidence in the effect estimate is limited; the true effect may be substantially different from the estimate of the effect.

Very low certainty: we have very little confidence in the effect estimate; the true effect is likely to be substantially different from the estimate of effect.

1 Downgraded one level for serious imprecision as only a single study with a small sample size and a wide $95 \% \mathrm{Cl}$.

2 Downgraded two levels for very serious imprecision as very wide $95 \% \mathrm{Cl}$ indicating the possibility of important benefit or harm.

Table 5. MAL-PDT compared to cryosurgery for basal cell carcinoma of the skin

\section{PDT compared to cryosurgery for low-risk BCC}

Patient or population: adults with basal cell carcinoma of the skin

Setting: secondary care with outpatients from France, UK, Sweden, Italy, Belgium and Austria

Intervention: MAL-PDT

Comparison: cryosurgery

\begin{tabular}{|c|c|c|c|c|c|c|}
\hline \multirow[t]{2}{*}{ Outcomes } & \multicolumn{2}{|c|}{ Anticipated absolute effects ${ }^{\star}(95 \% \mathrm{Cl})$} & \multirow{2}{*}{$\begin{array}{l}\text { Relative effect } \\
(95 \% \mathrm{CI})\end{array}$} & \multirow{2}{*}{$\begin{array}{l}\text { № of partici- } \\
\text { pants } \\
\text { (studies) }\end{array}$} & \multirow{2}{*}{$\begin{array}{l}\text { Certainty } \\
\text { of the ev- } \\
\text { idence } \\
\text { (GRADE) }\end{array}$} & \multirow[t]{2}{*}{ Comments } \\
\hline & $\begin{array}{l}\text { Risk with } \\
\text { cryosurgery }\end{array}$ & Risk with MAL-PDT & & & & \\
\hline \multirow{2}{*}{$\begin{array}{l}\text { Recurrence at } \\
3 \text { years }\end{array}$} & \multicolumn{2}{|c|}{ Study population } & \multirow{2}{*}{$\begin{array}{l}\text { RR } 1.14 \\
\text { (0.65 to } 1.98)\end{array}$} & \multirow{2}{*}{$\begin{array}{l}193 \text { (le- } \\
\text { sions) } \\
(1 \mathrm{RCT})\end{array}$} & \multirow{2}{*}{$\begin{array}{l}\oplus \oplus \odot \ominus \\
\text { LOW } 1\end{array}$} & \multirow[t]{2}{*}{-} \\
\hline & 194 per 1000 & $\begin{array}{l}221 \text { per } 1000 \\
\text { (126 to } 383)\end{array}$ & & & & \\
\hline \multirow{2}{*}{$\begin{array}{l}\text { Recurrence at } \\
5 \text { years }\end{array}$} & \multicolumn{2}{|c|}{ Study population } & \multirow{2}{*}{$\begin{array}{l}\text { RR } 1.08 \\
\text { (0.62 to } 1.86)\end{array}$} & \multirow{2}{*}{$\begin{array}{l}193 \text { (le- } \\
\text { sions) } \\
(1 \mathrm{RCT})\end{array}$} & \multirow{2}{*}{$\begin{array}{l}\oplus \oplus \odot \odot \\
\text { LOW } 1\end{array}$} & \multirow[t]{2}{*}{-} \\
\hline & 220 per 1000 & $\begin{array}{l}205 \text { per } 1000 \\
\text { (119 to } 352)\end{array}$ & & & & \\
\hline
\end{tabular}


Table 5. MAL-PDT compared to cryosurgery for basal cell carcinoma of the skin (Continued)

\begin{tabular}{|c|c|c|c|c|c|c|}
\hline \multirow{2}{*}{$\begin{array}{l}\text { Cosmetic out- } \\
\text { come (good/ } \\
\text { excellent) }\end{array}$} & \multicolumn{2}{|c|}{ Study population } & \multirow{2}{*}{$\begin{array}{l}\text { RR } 1.23 \\
(1.07 \text { to } 1.41)\end{array}$} & \multirow{2}{*}{$\begin{array}{l}99 \\
(1 \mathrm{RCT})\end{array}$} & \multirow{2}{*}{$\begin{array}{l}\oplus \oplus \oplus \odot \\
\text { MODER- } \\
\text { ATE } 2\end{array}$} & \multirow{2}{*}{$\begin{array}{l}\text { Participant-ratec } \\
\text { at } 1 \text { year on a } 4- \\
\text { point scale. }{ }^{2}\end{array}$} \\
\hline & 813 per 1000 & $\begin{array}{l}999 \text { per } 1000 \\
(869 \text { to } 1000)\end{array}$ & & & & \\
\hline \multirow{2}{*}{$\begin{array}{l}\text { Cosmetic out- } \\
\text { come (good/ } \\
\text { excellent) }\end{array}$} & Study popula & & \multirow{2}{*}{$\begin{array}{l}\text { RR } 1.46 \text { ( } 1.14 \text { to } \\
1.88)\end{array}$} & \multirow{2}{*}{$\begin{array}{l}99 \\
(1 \mathrm{RCT})\end{array}$} & \multirow{2}{*}{$\begin{array}{l}\oplus \oplus \oplus \ominus \\
\text { MODER- } \\
\text { ATE } 2\end{array}$} & \multirow{2}{*}{$\begin{array}{l}\text { Observer-rated } \\
\text { at } 1 \text { year on a } 4- \\
\text { point scale. }{ }^{2}\end{array}$} \\
\hline & 604 per 1000 & 882 per 1000 & & & & \\
\hline
\end{tabular}

(689 to 1000$)$

\begin{tabular}{|c|c|c|c|c|c|c|}
\hline \multirow{2}{*}{$\begin{array}{l}\text { Early treat- } \\
\text { ment failure }\end{array}$} & \multicolumn{2}{|c|}{ Study population } & \multirow{2}{*}{$\begin{array}{l}\text { RR } 0.57 \\
\text { (0.14 to } 2.33 \text { ) }\end{array}$} & \multirow{2}{*}{$\begin{array}{l}201 \text { (le- } \\
\text { sions) }{ }^{4} \\
(1 \mathrm{RCT})\end{array}$} & \multirow{2}{*}{$\begin{array}{l}\oplus \oplus \Theta \odot \\
\text { LOW } 1\end{array}$} & \multirow[t]{2}{*}{-} \\
\hline & 51 per 1000 & $\begin{array}{l}29 \text { per } 1000 \\
(7 \text { to } 119)\end{array}$ & & & & \\
\hline \multirow[t]{2}{*}{ Pain } & \multicolumn{2}{|c|}{ Study population } & \multirow{2}{*}{$\begin{array}{l}\text { RR } 1.12 \\
\text { (0.68 to } 1.84 \text { ) }\end{array}$} & \multirow{2}{*}{$\begin{array}{l}118 \\
(1 \mathrm{RCT})\end{array}$} & \multirow{2}{*}{$\begin{array}{l}\oplus \oplus \Theta \Theta \\
\text { LOW } 1\end{array}$} & \multirow{2}{*}{$\begin{array}{l}\text { Frequency re- } \\
\text { ported as AE dur- } \\
\text { ing the follow-up } \\
\text { period. }\end{array}$} \\
\hline & 328 per 1000 & $\begin{array}{l}367 \text { per } 1000 \\
\text { (223 to } 603)\end{array}$ & & & & \\
\hline
\end{tabular}

* The risk in the intervention group (and its $95 \%$ confidence interval) is based on the assumed risk in the comparison group and the relative effect of the intervention (and its $95 \% \mathrm{Cl}$ ).

AE: adverse event; Cl: Confidence interval; RR: Risk ratio.

\section{GRADE Working Group grades of evidence}

High certainty: we are very confident that the true effect lies close to that of the estimate of the effect.

Moderate certainty: we are moderately confident in the effect estimate; the true effect is likely to be close to the estimate of the effect, but there is a possibility that it is substantially different.

Low certainty: our confidence in the effect estimate is limited; the true effect may be substantially different from the estimate of the effect.

Very low certainty: we have very little confidence in the effect estimate; the true effect is likely to be substantially different from the estimate of effect.

1 Downgraded two levels for very serious imprecision as very wide $95 \% \mathrm{Cl}$ indicating the possibility of important benefit or harm.

2 Downgraded one level for serious risk of bias as unable to truly blind due to the nature of interventions.

3 4-point scale: poor (extensive occurrence of scarring, atrophy, or induration), fair (slight to moderate occurrence of scarring, atrophy or induration), good (no scarring, atrophy or induration and moderate redness or increase in pigmentation compared with adjacent skin), excellent (no scarring, atrophy, or induration and slight or no redness or change in pigmentation compared with adjacent skin).

4 Includes participants with multiple lesions which means differences between groups could be over-estimated.

\section{Table 6. 5-FU cream compared to MAL-PDT for basal cell carcinoma of the skin}

5-FU cream compared to MAL-PDT for low-risk BCC

Patient or population: adults with basal cell carcinoma of the skin

Setting: secondary care with outpatients from seven hospitals in the Netherlands

Intervention: 5-FU cream

Comparison: MAL-PDT

\begin{tabular}{|c|c|c|c|c|c|c|}
\hline \multirow[t]{2}{*}{ Outcomes } & \multicolumn{2}{|c|}{ Anticipated absolute effects ${ }^{\star}(95 \% \mathrm{Cl})$} & \multirow{2}{*}{$\begin{array}{l}\text { Relative ef- } \\
\text { fect } \\
(95 \% \mathrm{CI})\end{array}$} & \multirow{2}{*}{$\begin{array}{l}\text { № of } \\
\text { partici- } \\
\text { pants } \\
\text { (stud- } \\
\text { ies) }\end{array}$} & \multirow{2}{*}{$\begin{array}{l}\text { Certainty } \\
\text { of the ev- } \\
\text { idence } \\
\text { (GRADE) }\end{array}$} & \multirow[t]{2}{*}{ Comments } \\
\hline & Risk with PDT & Risk with 5-FU cream & & & & \\
\hline
\end{tabular}


Table 6. 5-FU cream compared to MAL-PDT for basal cell carcinoma of the skin (Continued)

\begin{tabular}{|c|c|c|c|c|c|c|}
\hline \multirow{2}{*}{$\begin{array}{l}\text { Recurrence at } \\
3 \text { years }\end{array}$} & \multicolumn{2}{|c|}{ Study population } & \multirow{2}{*}{$\begin{array}{l}\text { RR } 0.65 \\
(0.49 \text { to } 0.86)\end{array}$} & \multirow{2}{*}{$\begin{array}{l}272 \\
(1 \mathrm{RCT})\end{array}$} & \multirow{2}{*}{$\begin{array}{l}\oplus \oplus \oplus \ominus \\
\text { MODER- } \\
\text { ATE } 1\end{array}$} & \multirow[t]{2}{*}{-} \\
\hline & 524 per 1000 & $\begin{array}{l}340 \text { per } 1000 \\
(257 \text { to } 450)\end{array}$ & & & & \\
\hline \multirow{2}{*}{$\begin{array}{l}\text { Recurrence at } \\
5 \text { years }\end{array}$} & \multicolumn{2}{|c|}{ Study population } & \multirow{2}{*}{$\begin{array}{l}\text { RR } 0.67 \\
\text { (0.53 to } 0.84)\end{array}$} & \multirow{2}{*}{$\begin{array}{l}226 \\
(1 \mathrm{RCT})\end{array}$} & \multirow{2}{*}{$\begin{array}{l}\oplus \oplus \oplus \Theta \\
\text { MODER- } \\
\text { ATE } 1\end{array}$} & \multirow[t]{2}{*}{-} \\
\hline & 686 per 1000 & $\begin{array}{l}460 \text { per } 1000 \\
(364 \text { to } 576)\end{array}$ & & & & \\
\hline \multirow{2}{*}{$\begin{array}{l}\text { Cosmetic out- } \\
\text { come (excel- } \\
\text { lent/good) }\end{array}$} & \multicolumn{2}{|c|}{ Study population } & \multirow{2}{*}{$\begin{array}{l}\text { RR } 0.92 \\
\text { (0.78 to } 1.09)\end{array}$} & \multirow{2}{*}{$\begin{array}{l}379 \\
(1 \mathrm{RCT})\end{array}$} & \multirow{2}{*}{$\begin{array}{l}\oplus \oplus \oplus \odot \\
\text { MODER- } \\
\text { ATE }^{1}\end{array}$} & \multirow{2}{*}{$\begin{array}{l}\text { Blinded observer-rated at } 1 \\
\text { year on } 4 \text {-point scale. }{ }^{3}\end{array}$} \\
\hline & 624 per 1000 & $\begin{array}{l}574 \text { per } 1000 \\
(486 \text { to } 680)\end{array}$ & & & & \\
\hline \multirow{2}{*}{$\begin{array}{l}\text { Pain (moder- } \\
\text { ate/severe) }\end{array}$} & \multicolumn{2}{|c|}{ Study population } & \multirow{2}{*}{$\begin{array}{l}\text { RR } 0.41 \\
(0.26 \text { to } 0.63)\end{array}$} & \multirow{2}{*}{$\begin{array}{l}374 \\
(1 \mathrm{RCT})\end{array}$} & \multirow{2}{*}{$\begin{array}{l}\oplus \oplus \oplus \ominus \\
\text { MODER- } \\
\text { ATE } 1\end{array}$} & \multirow{2}{*}{$\begin{array}{l}\text { During treatment: com- } \\
\text { paring the week of treat- } \\
\text { ment with highest frequen- } \\
\text { cy of reported moderate/se- } \\
\text { vere pain (treatment cycle } \\
2 \text { for PDT, week } 4 \text { for } 5 \text {-FU } \\
\text { cream). }\end{array}$} \\
\hline & 305 per 1000 & $\begin{array}{l}125 \text { per } 1000 \\
(79 \text { to } 192)\end{array}$ & & & & \\
\hline \multirow{2}{*}{$\begin{array}{l}\text { Early treat- } \\
\text { ment failure }\end{array}$} & \multicolumn{2}{|c|}{ Study population } & \multirow{2}{*}{$\begin{array}{l}\text { RR } 0.77 \\
(0.47 \text { to } 1.26)\end{array}$} & \multirow{2}{*}{$\begin{array}{l}394 \\
(1 \mathrm{RCT})\end{array}$} & \multirow{2}{*}{$\begin{array}{l}\oplus \oplus \ominus \ominus \\
\text { LOW } 2\end{array}$} & \multirow[t]{2}{*}{-} \\
\hline & 158 per 1000 & $\begin{array}{l}122 \text { per } 1000 \\
\text { (74 to } 199)\end{array}$ & & & & \\
\hline
\end{tabular}

*The risk in the intervention group (and its $95 \%$ confidence interval) is based on the assumed risk in the comparison group and the relative effect of the intervention (and its $95 \% \mathrm{Cl}$ ).

Cl: Confidence interval; RR: Risk ratio.

\section{GRADE Working Group grades of evidence}

High certainty: we are very confident that the true effect lies close to that of the estimate of the effect.

Moderate certainty: we are moderately confident in the effect estimate; the true effect is likely to be close to the estimate of the effect, but there is a possibility that it is substantially different.

Low certainty: our confidence in the effect estimate is limited; the true effect may be substantially different from the estimate of the effect.

Very low certainty: we have very little confidence in the effect estimate; the true effect is likely to be substantially different from the estimate of effect.

1 Downgraded one level for serious imprecision as only a single study with a small sample size.

2 Downgraded two levels for very serious imprecision as very wide $95 \% \mathrm{Cl}$ indicating the possibility of important benefit or harm.

3 4-point scale: poor, fair, good, excellent.

Table 7. Imiquimod cream compared to 5-FU cream for basal cell carcinoma of the skin

\section{Imiquimod cream compared to 5-FU cream for low-risk BCC}

Patient or population: adults with basal cell carcinoma of the skin

Setting: secondary care with outpatients from seven hospitals in the Netherlands

Intervention: imiquimod cream

Comparison: 5-FU cream 
Table 7. Imiquimod cream compared to 5-FU cream for basal cell carcinoma of the skin (Continued)

\begin{tabular}{|c|c|c|c|c|c|c|}
\hline \multirow[t]{2}{*}{ Outcomes } & \multicolumn{2}{|c|}{ Anticipated absolute effects* $(95 \% \mathrm{Cl})$} & \multirow{2}{*}{$\begin{array}{l}\text { Relative ef- } \\
\text { fect } \\
(95 \% \mathrm{CI})\end{array}$} & \multirow{2}{*}{$\begin{array}{l}\text { № of } \\
\text { partici- } \\
\text { pants } \\
\text { (stud- } \\
\text { ies) }\end{array}$} & \multirow{2}{*}{$\begin{array}{l}\text { Certainty } \\
\text { of the ev- } \\
\text { idence } \\
\text { (GRADE) }\end{array}$} & \multirow[t]{2}{*}{ Comments } \\
\hline & $\begin{array}{l}\text { Risk with 5-FU } \\
\text { cream }\end{array}$ & $\begin{array}{l}\text { Risk with Imiquimod } \\
\text { cream }\end{array}$ & & & & \\
\hline \multirow{2}{*}{$\begin{array}{l}\text { Recurrence at } \\
3 \text { years }\end{array}$} & \multicolumn{2}{|l|}{ Study population } & \multirow{2}{*}{$\begin{array}{l}\text { RR } 0.68 \\
(0.47 \text { to } 0.99)\end{array}$} & \multirow{2}{*}{$\begin{array}{l}291 \\
(1 \mathrm{RCT})\end{array}$} & \multirow{2}{*}{$\begin{array}{l}\oplus \oplus \oplus \Theta \\
\text { MODER- } \\
\text { ATE } 1\end{array}$} & \multirow[t]{2}{*}{-} \\
\hline & 342 per 1000 & $\begin{array}{l}233 \text { per } 1000 \\
\text { (161 to } 339)\end{array}$ & & & & \\
\hline \multirow{2}{*}{$\begin{array}{l}\text { Recurrence at } \\
5 \text { years }\end{array}$} & \multicolumn{2}{|l|}{ Study population } & \multirow{2}{*}{$\begin{array}{l}\text { RR } 0.62 \\
\text { (0.44 to } 0.87)\end{array}$} & \multirow{2}{*}{$\begin{array}{l}250 \\
(1 \mathrm{RCT})\end{array}$} & \multirow{2}{*}{$\begin{array}{l}\oplus \oplus \oplus \ominus \\
\text { MODER- } \\
\text { ATE } 1\end{array}$} & \multirow[t]{2}{*}{-} \\
\hline & 460 per 1000 & $\begin{array}{l}285 \text { per } 1000 \\
(202 \text { to } 400)\end{array}$ & & & & \\
\hline \multirow{2}{*}{$\begin{array}{l}\text { Cosmetic out- } \\
\text { come (excel- } \\
\text { lent/good) }\end{array}$} & \multicolumn{2}{|l|}{ Study population } & \multirow{2}{*}{$\begin{array}{l}\text { RR } 1.07 \\
\text { (0.90 to } 1.26)\end{array}$} & \multirow{2}{*}{$\begin{array}{l}377 \\
(1 \mathrm{RCT})\end{array}$} & \multirow{2}{*}{$\begin{array}{l}\oplus \oplus \oplus \ominus \\
\text { MODER- } \\
\text { ATE }^{1}\end{array}$} & \multirow{2}{*}{$\begin{array}{l}\text { Observer-rated at } 1 \text { year } \\
\text { on } 4 \text {-point scale. }{ }^{4}\end{array}$} \\
\hline & 575 per 1000 & $\begin{array}{l}615 \text { per } 1000 \\
\text { (518 to } 725)\end{array}$ & & & & \\
\hline \multirow{2}{*}{$\begin{array}{l}\text { Pain (moder- } \\
\text { ate/severe) }\end{array}$} & \multicolumn{2}{|l|}{ Study population } & \multirow{2}{*}{$\begin{array}{l}\text { RR } 1.46 \\
\text { (0.89 to } 2.38 \text { ) }\end{array}$} & \multirow{2}{*}{$\begin{array}{l}365 \\
(1 \mathrm{RCT})\end{array}$} & \multirow{2}{*}{$\begin{array}{l}\oplus \oplus \oplus \ominus \\
\text { MODER- } \\
\text { ATE2 }^{2}\end{array}$} & \multirow{2}{*}{$\begin{array}{l}\text { During treatment: com- } \\
\text { paring the week of treat- } \\
\text { ment with highest fre- } \\
\text { quency of reported mod- } \\
\text { erate/severe pain (week } \\
6 \text { for imiquimod, week } 4 \\
\text { for } 5 \text {-FU cream). }\end{array}$} \\
\hline & 125 per 1000 & $\begin{array}{l}183 \text { per } 1000 \\
(111 \text { to } 298)\end{array}$ & & & & \\
\hline \multirow{2}{*}{$\begin{array}{l}\text { Early treat- } \\
\text { ment failure }\end{array}$} & \multicolumn{2}{|l|}{ Study population } & \multirow{2}{*}{$\begin{array}{l}\text { RR } 0.83 \\
(0.47 \text { to } 1.46)\end{array}$} & \multirow{2}{*}{$\begin{array}{l}387 \\
(1 \mathrm{RCT})\end{array}$} & \multirow{2}{*}{$\begin{array}{l}\oplus \oplus \ominus \ominus \\
\text { LOW } 3\end{array}$} & \multirow[t]{2}{*}{-} \\
\hline & 121 per 1000 & $\begin{array}{l}101 \text { per } 1000 \\
\text { (57 to } 177 \text { ) }\end{array}$ & & & & \\
\hline
\end{tabular}

*The risk in the intervention group (and its $95 \%$ confidence interval) is based on the assumed risk in the comparison group and the relative effect of the intervention (and its $95 \% \mathrm{Cl}$ ).

Cl: Confidence interval; RR: Risk ratio.

\section{GRADE Working Group grades of evidence}

High certainty: we are very confident that the true effect lies close to that of the estimate of the effect.

Moderate certainty: we are moderately confident in the effect estimate; the true effect is likely to be close to the estimate of the effect, but there is a possibility that it is substantially different.

Low certainty: our confidence in the effect estimate is limited; the true effect may be substantially different from the estimate of the effect.

Very low certainty: we have very little confidence in the effect estimate; the true effect is likely to be substantially different from the estimate of effect.

1 Downgraded one level for serious imprecision as only a single study with a small sample size.

2 Downgraded one level for serious imprecision as only a single study with a small sample size and a wide $95 \% \mathrm{Cl}$.

3 Downgraded two levels for very serious imprecision as very wide $95 \% \mathrm{Cl}$ indicating the possibility of important benefit or harm.

4 4-point scale: poor, fair, good, excellent.

Table 8. ALA-PDT compared to cryosurgery for basal cell carcinoma of the skin

PDT compared to cryosurgery for low-risk BCC 
Table 8. ALA-PDT compared to cryosurgery for basal cell carcinoma of the skin (Continued)

Patient or population: adults with basal cell carcinoma of the skin

Setting: secondary care with outpatients from a single hospital in Sweden

Intervention: ALA-PDT

Comparison: cryosurgery

\begin{tabular}{|c|c|c|c|c|c|c|}
\hline \multirow[t]{2}{*}{ Outcomes } & \multicolumn{2}{|c|}{ Anticipated absolute effects ${ }^{\star}(95 \% \mathrm{CI})$} & \multirow{2}{*}{$\begin{array}{l}\text { Relative } \\
\text { effect } \\
(95 \% \mathrm{CI})\end{array}$} & \multirow{2}{*}{$\begin{array}{l}\text { № of } \\
\text { par- } \\
\text { tici- } \\
\text { pants } \\
\text { (stud- } \\
\text { ies) }\end{array}$} & \multirow{2}{*}{$\begin{array}{l}\text { Cer- } \\
\text { tain- } \\
\text { ty of } \\
\text { the ev- } \\
\text { idence } \\
\text { (GRADE) }\end{array}$} & \multirow[t]{2}{*}{ Comments } \\
\hline & $\begin{array}{l}\text { Risk with } \\
\text { cryosurgery }\end{array}$ & Risk with ALA-PDT & & & & \\
\hline $\begin{array}{l}\text { Recurrence at } \\
3 \text { years }\end{array}$ & \multicolumn{2}{|c|}{ No study addressed this outcome. } & $\begin{array}{l}\text { not es- } \\
\text { timable }\end{array}$ & - & - & $\begin{array}{l}\text { No study addressed this out- } \\
\text { come. }\end{array}$ \\
\hline $\begin{array}{l}\text { Recurrence at } \\
5 \text { years }\end{array}$ & \multicolumn{2}{|c|}{ No study addressed this outcome. } & $\begin{array}{l}\text { not es- } \\
\text { timable }\end{array}$ & - & - & $\begin{array}{l}\text { No study addressed this out- } \\
\text { come. }\end{array}$ \\
\hline \multirow{2}{*}{$\begin{array}{l}\text { Cosmetic out- } \\
\text { come (good/ } \\
\text { excellent) }\end{array}$} & \multicolumn{2}{|c|}{ Study population } & \multirow{2}{*}{$\begin{array}{l}\text { RR } 1.72 \\
(1.26 \text { to } \\
2.34)\end{array}$} & \multirow{2}{*}{$\begin{array}{l}79 \\
(1 \\
\mathrm{RCT})\end{array}$} & \multirow{2}{*}{$\begin{array}{l}\oplus \oplus \oplus \ominus \\
\text { MOD- } \\
\text { ERATE } \\
1\end{array}$} & \multirow{2}{*}{$\begin{array}{l}\text { Observer-rated at } 1 \text { year on a } 4 \\
\text { point scale. }{ }^{3}\end{array}$} \\
\hline & 541 per 1000 & $\begin{array}{l}930 \text { per } 1000 \text { (681 to } \\
1000)\end{array}$ & & & & \\
\hline $\begin{array}{l}\text { Early treat- } \\
\text { ment failure }\end{array}$ & No study addre & this outcome. & $\begin{array}{l}\text { not es- } \\
\text { timable }\end{array}$ & - & - & - \\
\hline \multirow[t]{2}{*}{ Pain } & \multicolumn{2}{|c|}{ Study population } & \multirow{2}{*}{$\begin{array}{l}\text { not es- } \\
\text { timable }\end{array}$} & \multirow{2}{*}{$\begin{array}{l}88 \\
(1 \\
\mathrm{RCT})\end{array}$} & \multirow{2}{*}{$\begin{array}{l}\oplus \oplus \oplus \ominus \\
\text { MOD- } \\
\text { ERATE } \\
2\end{array}$} & \multirow{2}{*}{$\begin{array}{l}\text { Pain during treatment assessed } \\
\text { by VAS ( } 115 \mathrm{~mm} \text {, where } 115 \mathrm{~mm} \\
\text { corresponds to 'unbearable } \\
\text { pain' and } 0 \mathrm{~mm} \text { corresponds to } \\
\text { 'no pain'. }\end{array}$} \\
\hline & $\begin{array}{l}\text { The mean pain } \\
\text { was } 32 \mathrm{~mm}\end{array}$ & $\begin{array}{l}\text { MD } 11 \mathrm{~mm} \text { higher ( } 1.12 \\
\text { lower to } 23.12 \text { higher) }\end{array}$ & & & & \\
\hline
\end{tabular}

*The risk in the intervention group (and its 95\% confidence interval) is based on the assumed risk in the comparison group and the relative effect of the intervention (and its $95 \% \mathrm{Cl}$ ).

Cl: Confidence interval; RR: Risk ratio; VAS: visual analogue scale.

GRADE Working Group grades of evidence

High certainty: we are very confident that the true effect lies close to that of the estimate of the effect.

Moderate certainty: we are moderately confident in the effect estimate; the true effect is likely to be close to the estimate of the effect, but there is a possibility that it is substantially different.

Low certainty: our confidence in the effect estimate is limited; the true effect may be substantially different from the estimate of the effect.

Very low certainty: we have very little confidence in the effect estimate; the true effect is likely to be substantially different from the estimate of effect.

1 Downgraded one level for serious risk of bias as unable to truly blind due to the nature of interventions.

2 Downgraded one level for serious imprecision as only a single study with a small sample size and a wide $95 \% \mathrm{Cl}$.

3 4-point scale: blemished, acceptable, good, excellent. 


\section{AP PE N D I E S}

\section{Appendix 1. Skin Group Specialised Register (CRS) search strategy}

(basal cell* and (cancer* or epithelioma* or carcinoma* or naev* or nev* or tumor* or tumour* or neoplasm*)) or bcc or "gorlin syndrome" or ((rodent or Jacob*) and ulcer*) or basalioma or nmsc or (keratinocyte ${ }^{\star}$ and (cancer ${ }^{\star}$ or carcinoma*)) or nonmelanoma or "non melanoma" or "basal keratinocyte*"

\section{Appendix 2. CENTRAL (Cochrane Library) search strategy}

\#1 MeSH descriptor: [Carcinoma, Basal Cell] explode all trees \#2 MeSH descriptor: [Neoplasms, Basal Cell] explode all trees \#3 MeSH descriptor: [Basal Cell Nevus Syndrome] explode all trees

\#4 (basal cell* next (cancer* or epithelioma* or carcinoma* or naev* or nev* or tumor ${ }^{\star}$ or tumour* or neoplasm)):ti,ab,kw \#5 bcc:ti,ab,kw

\#6 gorlin syndrome:ti,ab,kw

\#7 (rodent next ulcer $\left.{ }^{\star}\right)$ :ti,ab,kw

\#8 (jacob* next ulcer $\left.{ }^{\star}\right): t i, a b, k w$

\#9 basalioma*:ti,ab,kw

$\# 10$ nmsc:ti,ab,kw

\#11 (keratinocyte* and (cancer* or carcinoma*)):ti,ab,kw

\#12 nonmelanoma skin cancer*:ti,ab,kw

\#13 non melanoma skin cancer ${ }^{\star}:$ ti,ab,kw

$\# 14$ basal keratinocyte*:ti,ab,kw

$\# 15$ or \#1-\#14\}

\section{Appendix 3. MEDLINE (Ovid) search strategy}

1. randomized controlled trial.pt.

2. controlled clinical trial.pt.

3. randomized.ab.

4. placebo.ab.

5. clinical trials as topic.sh.

6. randomly.ab.

7. trial.ti.

8. or/1-7

9. exp animals/ not humans.sh.

10. 8 not 9

11. exp Carcinoma, Basal Cell/

12. (basal cell\$ and (cancer\$ or epithelioma\$ or carcinoma\$ or naev\$ or nev\$ or tumor\$ or tumour $\$$ or neoplasm\$)).mp.

13. exp Neoplasms, Basal Cell/

14. bcc.ti,ab.

15. gorlin syndrome.mp.

16. Basal Cell Nevus Syndrome/

17. rodent ulcer\$.mp.

18. jacob\$ ulcer\$.mp.

19. basalioma\$.mp.

20. nmsc.ti,ab.

21. (keratinocyte\$ and (cancer\$ or carcinoma\$)).mp.

22. nonmelanoma skin cancer\$.mp.

23. non melanoma skin cancer\$.mp.

24. basal keratinocyte\$.mp.

25. or/11-24

26. 10 and 25

[Lines 1-10: Cochrane Highly Sensitive Search Strategy for identifying randomized trials in MEDLINE: sensitivity-and precision-maximizing version (2008 revision); Ovid format, from section 3.6.1 in Lefebvre C, Glanville J, Briscoe S, Littlewood A, Marshall C, Metzendorf MI, et al. Technical Supplement to Chapter 4: Searching for and selecting studies. In: Higgins JPT, Thomas J, Chandler J, Cumpston MS, Li T, Page MJ, Welch VA (eds). Cochrane Handbook for Systematic Reviews of Interventions Version 6. Cochrane, 2019. Available from: www.training.cochrane.org/handbook]

\section{Appendix 4. Embase (Ovid) search strategy}

1. exp Basal Cell Carcinoma/ 
2. (basal cell\$ and (cancer\$ or epithelioma\$ or carcinoma\$ or naev\$ or nev\$ or tumor\$ or tumour\$ or neoplasm\$)).mp.

3. bcc.ti,ab.

4. gorlin syndrome.mp.

5. exp Basal Cell Nevus Syndrome/

6. rodent ulcer\$.mp.

7. jacob\$ ulcer\$.mp.

8. basalioma\$.mp.

9. (keratinocyte\$ and (cancer\$ or carcinoma\$)).mp.

10. nonmelanoma skin cancer\$.mp.

11. non melanoma skin cancer\$.mp.

12. basal keratinocyte\$.mp.

13. nmsc.ti,ab.

14. or/1-13

15. crossover procedure.sh.

16. double-blind procedure.sh.

17. single-blind procedure.sh.

18. (crossover\$ or cross over\$).tw.

19. placebo\$.tw.

20. (doubl\$ adj blind\$).tw.

21. allocat\$.tw.

22. trial.ti.

23. randomized controlled trial.sh.

24. random $\$$.tw.

25. or/15-24

26. exp animal/ or exp invertebrate/ or animal experiment/ or animal model/ or animal tissue/ or animal cell/ or nonhuman/

27. human/ or normal human/

28. 26 and 27

29. 26 not 28

30.25 not 29

31.14 and 30

[Lines 15-25: Based on terms suggested for identifying RCTs in Embase (section 3.6.2) in Lefebvre C, Glanville J, Briscoe S, Littlewood A, Marshall C, Metzendorf M-I, et al. Technical Supplement to Chapter 4: Searching for and selecting studies. In: Higgins JPT, Thomas J, Chandler J, Cumpston MS, Li T, Page MJ, Welch VA (eds). Cochrane Handbook for Systematic Reviews of Interventions Version 6 . Cochrane, 2019. Available from: www.training.cochrane.org/handbook]

\section{Appendix 5. CINAHL (EBSCO) search strategy}

S1TI basal cell and (cancer* or epithelioma* or carcinoma* or naev* or nev* or tumor ${ }^{\star}$ or tumour ${ }^{\star}$ or neoplasm*)) or AB basal cell and (cancer ${ }^{\star}$ or epithelioma* or carcinoma* or naev* or nev* or tumor ${ }^{\star}$ or tumour ${ }^{\star}$ or neoplasm $\left.{ }^{\star}\right)$ )

S2 (MH "Carcinoma, Basal Cell") OR (MH "Neoplasms, Basal Cell+")

S3 TI ((non melanoma or nonmelanoma) and skin cancer ${ }^{\star}$ ) or AB ((non melanoma or nonmelanoma) and skin cancer $)$

S4 "gorlin syndrome"

S5 Jacob* nr1 ulcer*

S6 (rodent next ulcer ${ }^{\star}$ )

S7 TI bcc or AB bcc

S8 TX basalioma*

S9 TI nmsc or AB nmsc

S10 TX (keratinocyte ${ }^{\star}$ nr2 (cancer* or carcinoma $\left.\left.^{\star}\right)\right)$

S11TI basal keratinocyte* or AB basal keratinocyte*

S12 TX random*

S13 S1 OR S2 OR S3 OR S4 OR S5 OR S6 OR S7 OR S8 OR S9 OR S10 OR S11

S14 S12 AND S13

\section{Appendix 6. LILACS search strategy}

Searched on the advanced search page with ti and ab fields only, and using the Controlled clinical trials topic-specific query filter. ("basal cell" or "basal cells" or "rodent ulcer" or "rodent ulcers" or "jacobs ulcer" or "jacobs ulcers" or basalioma or nmsc or "non melanoma skin cancer" or "non melanoma skin cancers"):ti,ab

\section{WHAT'S NEW}




\begin{tabular}{lll}
\hline Date & Event & Description \\
\hline 9 September 2020 & $\begin{array}{l}\text { New citation required and conclusions } \\
\text { have changed }\end{array}$ & $\begin{array}{l}\text { This update included studies of many more interventions, with } \\
\text { several new studies of non-surgical interventions compared to } \\
\text { surgical interventions and other non-surgical interventions. It al- } \\
\text { so includes long-term follow-up data from previously included } \\
\text { studies. These new data have led to new and updated conclu- } \\
\text { sions. }\end{array}$ \\
& \\
& & \\
& &
\end{tabular}

19 February $2020 \quad$ New search has been performed

Since the 2007 review, we have identified 26 new randomised controlled trials. This updated review now has a total of 52 studies that randomised 6690 participants.

\section{HIST ORY}

Protocol first published: Issue 1, 2002

Review first published: Issue 2, 2003

\begin{tabular}{lll}
\hline Date & Event & Description \\
\hline 22 May 2008 & Amended & Converted to new review format. \\
\hline 9 November 2006 & $\begin{array}{l}\text { New citation required and conclusions } \\
\text { have changed }\end{array}$ & Substantive amendment \\
\hline
\end{tabular}

\section{CONTRIBUTIONS OFAUTHORS}

JT was the contact person with the editorial base, JT co-ordinated contributions from the co-authors, and wrote the final draft of the review. JT, FB-H, and SH screened papers against eligibility criteria.

JT, FB-H, and SH obtained data on ongoing and unpublished studies.

JT, FB-H, and SH appraised the quality of papers.

JT, FB-H, and SH extracted data for the review and sought additional information about papers.

JT, FB-H, and SH entered data into RevMan.

FB-H, JT, SH and JL-B analysed and interpreted data.

$\mathrm{FB}-\mathrm{H}$ and JT worked on the methods sections.

JT and HW drafted the clinical sections of the background and responded to the clinical comments of the referees.

$\mathrm{JL}-\mathrm{B}$ responded to the methodology and statistics comments of the referees.

JT is the guarantor of the update.

\section{Disclaimer}

This project was supported by the National Institute for Health Research (NIHR), via Cochrane Infrastructure funding to the Cochrane Skin Group. The views and opinions expressed therein are those of the authors and do not necessarily reflect those of the Systematic Reviews Programme, NIHR, National Health Service (NHS), or the Department of Health.

\section{DECLARATIONS OF INTEREST}

Jason Thomson: none known

Sarah Hogan: none known

Jo Leonardi-Bee: none known

Hywel C Williams and Fiona J Bath-Hextall were involved in the SINS trial that compared topical imiquimod versus surgery and is a trial that is included in this review (Bath-Hextall 2014). The trial was funded by Cancer Research UK, a UK cancer charity. They were not involved in extracting data from the trial nor commenting on the evidence from this trial. Neither the charity, Fiona J Bath-Hextall or Hywel Williams have any links with industry. 


\section{SOURCESOF SUPPORT}

\section{Internal sources}

- Cochrane Skin, UK

\section{External sources}

- The National Institute for Health Research (NIHR), UK

The NIHR, UK, is the largest single funder of Cochrane Skin.

\section{DIFFERENCES BETWEEN PROTOCOLANDREVIEW}

We have amended the primary and secondary outcomes of this updated review to reflect the outcomes that are important for both clinicians and patients as well as to conform to the recommendations from Cochrane. Specifically, we have separated "recurrence at 3 to 5 years" to "recurrence at 3 years" and "recurrence at 5 years". The reason for this was because " 3 to 5 years" is a wide time range and we did not feel it would be appropriate to compare three-year outcomes with five-year outcomes. This was not such a concern for the 2007 review as very few studies had long term follow-up data, but for this review we were aware that many more studies would have followup data up to five years.

We have also amended the cosmetic outcome. In the 2007 review this outcome was "Aesthetic appearance to participants/observer: atrophy, scarring, changes in pigmentation" and was categorised as an "adverse effect". We did not feel this was appropriate and have changed this to "cosmetic outcome (participant- and observer-rated)", and after discussion with HW, who had not seen the results, if multiple time points were reported, we included the time point closest to one year (but not less than one year) following treatment, as this is the minimum time taken for a scar to mature. Additionally, on Cochrane Skin's advice we have made the cosmetic outcome a primary outcome so as to include both a benefit and harm as primary outcomes.

We have renamed the secondary outcome "discomfort to participants in terms of pain during treatment and thereafter" to "pain during treatment and thereafter" to make it clearer and more specific. All adverse effects data were recorded but due to the heterogeneous nature of these data and the way they were presented, we did not perform analyses or present them in the 'Summary of findings' tables. Instead we collated all these into a separate adverse effects table.

In methods, a section has been added detailing the addition of GRADE assessments of the certainty of evidence as well as 'Summary of findings' tables and how they were produced.

Due to the large number of studies and to improve readability of the review, we excluded studies that did not measure any of our outcomes of interest in accordance with MECIR C40.

We decided to include studies that compared an active treatment against a no treatment arm. The reason we departed from the protocol on this was because we did not have a strong justification for excluding such studies and we were challenged on this by two of our peerreviewers.

JT, SH and J-LB are new authors since the last review. JB and WB are not authors on this update.

\section{N DEX TERMS}

\section{Medical Subject Headings (MeSH)}

Antineoplastic Agents [therapeutic use]; Carcinoma, Basal Cell [surgery] [*therapy]; Cryotherapy; Photochemotherapy; Radiotherapy; Randomized Controlled Trials as Topic; Skin Neoplasms [surgery] [therapy]; Treatment Outcome

\section{MeSH check words}

Adult; Humans 